\title{
A Decade of Gamma-Ray Bursts Observed by Fermi-LAT: The Second GRB Catalog
}

\author{
M. Ajello ${ }^{1}$, M. Arimoto ${ }^{2}$, M. Axelsson ${ }^{3,4}$ (1) L. Baldini ${ }^{5}$, G. Barbiellini ${ }^{6,7}$, D. Bastieri ${ }^{8,9}$, R. Bellazzini ${ }^{10}$, P. N. Bhat ${ }^{11}$,
} E. Bissaldi ${ }^{12,13}$ (1) R. D. Blandford ${ }^{14}$, R. Bonino ${ }^{15,16}$, J. Bonnell ${ }^{17,18}$, E. Bottacini ${ }^{14,19}{ }^{\text {, J. Bregeon }}{ }^{20}$, P. Bruel ${ }^{21}$, R. Buehler ${ }^{22}$, R. A. Cameron ${ }^{14}$, R. Caputo ${ }^{23}$, P. A. Caraveo ${ }^{24}$, E. Cavazzuti ${ }^{25}$, S. Chen ${ }^{8,19}$, C. C. Cheung ${ }^{26}$, G. Chiaro ${ }^{24}$, S. Ciprini ${ }^{27,28}$, D. Costantin ${ }^{29}$, M. Crnogorcevic ${ }^{18}$, S. Cutini ${ }^{30}$, M. Dainotti ${ }^{14}$, F. D'Ammando ${ }^{31,32}$, P. de la Torre Luque ${ }^{12}$, F. de Palma ${ }^{15}$, A. Desai ${ }^{1}$, R. Desiante ${ }^{15}$, N. Di Lalla ${ }^{5}$, L. Di Venere ${ }^{12,13}$, F. Fana Dirirsa ${ }^{33}$, S. J. Fegan ${ }^{21}$, A. Franckowiak ${ }^{22}$, Y. Fukazawa ${ }^{34}$, S. Funk ${ }^{35}$, P. Fusco ${ }^{12,13}$, F. Gargano ${ }^{13}$, D. Gasparrini ${ }^{28,30}$, N. Giglietto ${ }^{12,13}$, F. Giordano ${ }^{12,13}$, M. Giroletti ${ }^{31}$, D. Green ${ }^{36}$, I. A. Grenier ${ }^{37}$, J. E. Grove ${ }^{26}$, S. Guiriec ${ }^{17,38}$, E. Hays ${ }^{17}$, J. W. Hewitt ${ }^{39}$ (1), D. Horan ${ }^{21}$, G. Jóhannesson ${ }^{40,41}$, D. Kocevski ${ }^{17}$, M. Kuss ${ }^{10}$, L. Latronico ${ }^{15}$, J. Li ${ }^{22}$, F. Longo ${ }^{6,7}$, F. Loparco ${ }^{12,13}$, M. N. Lovellette ${ }^{26}$, P. Lubrano ${ }^{30}$, S. Maldera ${ }^{15}$ (D) A. Manfreda ${ }^{5}$, G. Martí-Devesa $^{42}$, M. N. Mazziotta ${ }^{13}$, I. Mereu ${ }^{43}$, M. Meyer ${ }^{14}$, P. F. Michelson ${ }^{14}$, N. Mirabal ${ }^{17,44}$, W. Mitthumsiri ${ }^{45}$, T. Mizuno $^{46}$, M. E. Monzani ${ }^{14}$, E. Moretti ${ }^{47}$, A. Morselli ${ }^{27}$, I. V. Moskalenko ${ }^{14}$, M. Negro ${ }^{15,16}$, E. Nuss ${ }^{20}$, M. Ohno ${ }^{34}$, N. Omodei ${ }^{14}$ (1),

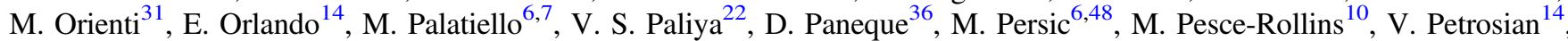
F. Piron ${ }^{20}$, S. Poolakkil ${ }^{11}$, H. Poon ${ }^{34}$, T. A. Porter ${ }^{14}$, G. Principe ${ }^{35}$, J. L. Racusin ${ }^{17}$, S. Rainò ${ }^{12,13}$, R. Rando ${ }^{8,9}$, M. Razzano ${ }^{10,61}$, S. Razzaque ${ }^{33}$, A. Reimer ${ }^{14,42}$, O. Reimer ${ }^{14,42}$, T. Reposeur ${ }^{49}$, F. Ryde ${ }^{4,50}$, D. Serini ${ }^{12}$, C. Sgrò ${ }^{10}$, E. J. Siskind ${ }^{51}$, E. Sonbas ${ }^{52}$, G. Spandre ${ }^{10}$, P. Spinelli ${ }^{12,13}$, D. J. Suson ${ }^{53}$, H. Tajima ${ }^{14,54}$, M. Takahashi ${ }^{36}$, D. Tak ${ }^{17,18}$, J. B. Thayer ${ }^{14}$, D. F. Torres ${ }^{55,56}$, E. Troja ${ }^{17,18}$, J. Valverde ${ }^{21}$, P. Veres ${ }^{11}$, G. Vianello ${ }^{14}$ (1) A. von Kienlin ${ }^{57}$, K. Wood ${ }^{58}$, M. Yassine ${ }^{6,7}$, S. Zhu ${ }^{59}$, and S. Zimmer ${ }^{42,60}$ ${ }^{1}$ Department of Physics and Astronomy, Clemson University, Kinard Lab of Physics, Clemson, SC 29634-0978, USA

${ }^{2}$ Faculty of Mathematics and Physics, Institute of Science and Engineering, Kanazawa University, Kakuma, Kanazawa, Ishikawa 920-1192, Japan

${ }^{3}$ Department of Physics and Oskar Klein Center for Cosmoparticle Physics, Stockholm University, SE-106 91 Stockholm, Sweden; magnusa@astro.su.se ${ }^{4}$ Department of Physics, KTH Royal Institute of Technology, AlbaNova, SE-106 91 Stockholm, Sweden

${ }^{5}$ Università di Pisa and Istituto Nazionale di Fisica Nucleare, Sezione di Pisa I-56127 Pisa, Italy

${ }^{6}$ Istituto Nazionale di Fisica Nucleare, Sezione di Trieste, I-34127 Trieste, Italy

${ }^{7}$ Dipartimento di Fisica, Università di Trieste, I-34127 Trieste, Italy

${ }^{8}$ Istituto Nazionale di Fisica Nucleare, Sezione di Padova, I-35131 Padova, Italy

9 Dipartimento di Fisica e Astronomia "G. Galilei," Università di Padova, I-35131 Padova, Italy

${ }^{10}$ Istituto Nazionale di Fisica Nucleare, Sezione di Pisa, I-56127 Pisa, Italy

${ }^{11}$ Center for Space Plasma and Aeronomic Research (CSPAR), University of Alabama in Huntsville, Huntsville, AL 35899, USA

${ }^{12}$ Dipartimento di Fisica "M. Merlin” dell’Università e del Politecnico di Bari, I-70126 Bari, Italy; elisabetta.bissaldi@ba.infn.it

${ }^{13}$ Istituto Nazionale di Fisica Nucleare, Sezione di Bari, I-70126 Bari, Italy

${ }^{14}$ W.W. Hansen Experimental Physics Laboratory, Kavli Institute for Particle Astrophysics and Cosmology, Department of Physics and SLAC National Accelerator Laboratory, Stanford University, Stanford, CA 94305, USA; nicola.omodei@ stanford.edu, giacomov@ slac.stanford.edu

${ }_{16}^{15}$ Istituto Nazionale di Fisica Nucleare, Sezione di Torino, I-10125 Torino, Italy

${ }^{16}$ Dipartimento di Fisica, Università degli Studi di Torino, I-10125 Torino, Italy 7 NASA Goddard Space Flight Center, Greenbelt, MD 20771, USA

${ }^{18}$ Department of Astronomy, University of Maryland, College Park, MD 20742, USA

${ }^{19}$ Department of Physics and Astronomy, University of Padova, Vicolo Osservatorio 3, I-35122 Padova, Italy

${ }^{20}$ Laboratoire Univers et Particules de Montpellier, Université Montpellier, CNRS/IN2P3, F-34095 Montpellier, France

${ }^{21}$ Laboratoire Leprince-Ringuet, École polytechnique, CNRS/IN2P3, F-91128 Palaiseau, France

${ }^{22}$ Deutsches Elektronen Synchrotron DESY, D-15738 Zeuthen, Germany

${ }^{23}$ Center for Research and Exploration in Space Science and Technology (CRESST) and NASA Goddard Space Flight Center, Greenbelt, MD 20771, USA

${ }^{24}$ INAF-Istituto di Astrofisica Spaziale e Fisica Cosmica Milano, via E. Bassini 15, I-20133 Milano, Italy

${ }^{25}$ Italian Space Agency, Via del Politecnico snc, I-00133 Roma, Italy

${ }^{26}$ Space Science Division, Naval Research Laboratory, Washington, DC 20375-5352, USA

${ }^{27}$ Istituto Nazionale di Fisica Nucleare, Sezione di Roma "Tor Vergata," I-00133 Roma, Italy

${ }^{28}$ Space Science Data Center-Agenzia Spaziale Italiana, Via del Politecnico, snc, I-00133, Roma, Italy

${ }^{29}$ University of Padua, Department of Statistical Science, Via 8 Febbraio, 2, I-35122 Padova, Italy

${ }^{30}$ Istituto Nazionale di Fisica Nucleare, Sezione di Perugia, I-06123 Perugia, Italy

${ }^{31}$ INAF Istituto di Radioastronomia, I-40129 Bologna, Italy

${ }^{32}$ Dipartimento di Astronomia, Università di Bologna, I-40127 Bologna, Italy

${ }_{33}$ Department of Physics, University of Johannesburg, P.O. Box 524, Auckland Park 2006, South Africa

${ }^{35}$ Friedrich-Alexander-Universität Erlangen-Nürnberg, Erlangen Centre for Astroparticle Physics, Erwin-Rommel-Str. 1, D-91058 Erlangen, Germany

${ }^{36}$ Max-Planck-Institut für Physik, D-80805 München, Germany

${ }^{37}$ AIM, CEA, CNRS, Université Paris-Saclay, Université Paris Diderot, Sorbonne Paris Cité, F-91191 Gif-sur-Yvette, France

${ }^{2}$ The George Washington University, Department of Physics, 725 21st St., NW, Washington, DC 20052, USA

${ }^{39}$ University of North Florida, Department of Physics, 1 UNF Drive, Jacksonville, FL 32224, USA

${ }^{40}$ Science Institute, University of Iceland, IS-107 Reykjavik, Iceland

${ }^{41}$ Nordita, Royal Institute of Technology and Stockholm University, Roslagstullsbacken 23, SE-106 91 Stockholm, Sweden

${ }^{42}$ Institut für Astro- und Teilchenphysik and Institut für Theoretische Physik, Leopold-Franzens-Universität Innsbruck, A-6020 Innsbruck, Austria

${ }^{43}$ Dipartimento di Fisica, Università degli Studi di Perugia, I-06123 Perugia, Italy

${ }^{44}$ Department of Physics and Center for Space Sciences and Technology, University of Maryland Baltimore County, Baltimore, MD 21250, USA

${ }^{45}$ Department of Physics, Faculty of Science, Mahidol University, Bangkok 10400, Thailand

${ }^{46}$ Hiroshima Astrophysical Science Center, Hiroshima University, Higashi-Hiroshima, Hiroshima 739-8526, Japan

${ }^{47}$ Institut de Física d'Altes Energies (IFAE), Edifici Cn, Universitat Autònoma de Barcelona (UAB), E-08193 Bellaterra (Barcelona), Spain

${ }^{48}$ Osservatorio Astronomico di Trieste, Istituto Nazionale di Astrofisica, I-34143 Trieste, Italy

${ }^{49}$ Centre d'Études Nucléaires de Bordeaux Gradignan, IN2P3/CNRS, Université Bordeaux 1, BP120, F-33175 Gradignan Cedex, France

${ }^{50}$ The Oskar Klein Centre for Cosmoparticle Physics, AlbaNova, SE-106 91 Stockholm, Sweden 


\author{
${ }^{51}$ NYCB Real-Time Computing Inc., Lattingtown, NY 11560-1025, USA \\ 52 Adıyaman University, 02040 Adiyaman, Turkey \\ ${ }^{53}$ Purdue University Northwest, Hammond, IN 46323, USA \\ ${ }^{54}$ Solar-Terrestrial Environment Laboratory, Nagoya University, Nagoya 464-8601, Japan \\ ${ }^{55}$ Institute of Space Sciences (CSICIEEC), Campus UAB, Carrer de Magrans s/n, E-08193 Barcelona, Spain \\ ${ }^{56}$ Institució Catalana de Recerca i Estudis Avançats (ICREA), E-08010 Barcelona, Spain \\ ${ }^{57}$ Max-Planck Institut für extraterrestrische Physik, D-85748 Garching, Germany
${ }^{58}$ Praxis Inc., Alexandria, VA 22303, resident at Naval Research Laboratory, Washington, DC 20375, USA \\ ${ }^{59}$ Albert-Einstein-Institut, Max-Planck-Institut für Gravitationsphysik, D-30167 Hannover, Germany \\ ${ }^{60}$ University of Geneva, Département de physique nucléaire et corpusculaire (DPNC), 24 quai Ernest-Ansermet, CH-1211 Genève 4, Switzerland \\ Received 2019 March 28; accepted 2019 April 25; published 2019 June 13
}

\begin{abstract}
The Large Area Telescope (LAT) aboard the Fermi spacecraft routinely observes high-energy emission from gamma-ray bursts (GRBs). Here we present the second catalog of LAT-detected GRBs, covering the first $10 \mathrm{yr}$ of operations, from 2008 to 2018 August 4. A total of 186 GRBs are found; of these, 91 show emission in the range 30-100 MeV (17 of which are seen only in this band) and 169 are detected above $100 \mathrm{MeV}$. Most of these sources were discovered by other instruments (Fermi/GBM, Swift/BAT, AGILE, INTEGRAL) or reported by the Interplanetary Network (IPN); the LAT has independently triggered on four GRBs. This catalog presents the results for all 186 GRBs. We study onset, duration, and temporal properties of each GRB, as well as spectral characteristics in the $100 \mathrm{MeV}-100 \mathrm{GeV}$ energy range. Particular attention is given to the photons with the highest energy. Compared with the first LAT GRB catalog, our rate of detection is significantly improved. The results generally confirm the main findings of the first catalog: the LAT primarily detects the brightest GBM bursts, and the high-energy emission shows delayed onset as well as longer duration. However, in this work we find delays exceeding $1 \mathrm{ks}$ and several GRBs with durations over $10 \mathrm{ks}$. Furthermore, the larger number of LAT detections shows that these GRBs not only cover the high-fluence range of GBM-detected GRBs but also sample lower fluences. In addition, the greater number of detected GRBs with redshift estimates allows us to study their properties in both the observer and rest frames. Comparison of the observational results with theoretical predictions reveals that no model is currently able to explain all results, highlighting the role of LAT observations in driving theoretical models.
\end{abstract}

Key words: catalogs - gamma-ray burst: general - methods: data analysis

Supporting material: FITS file

\section{Introduction}

Observations by the Fermi Gamma-Ray Space Telescope in the $10 \mathrm{yr}$ since it was placed into orbit on 2008 June 11 have allowed for the opportunity to study the broadband properties of gamma-ray bursts (GRBs) over an unprecedented energy range. Its two scientific instruments, the Large Area Telescope (LAT; Atwood et al. 2009) and the Gamma-Ray Burst Monitor (GBM; Meegan et al. 2009), provide the combined capability of probing emission from GRBs over seven decades in energy. These groundbreaking observations have helped to characterize the highest-energy emission from these events (Abdo et al. 2009a, 2009e, 2010a; Ackermann et al. 2010a, 2010b, 2011), furthered our understanding of the emission processes associated with GRBs (Ryde et al. 2010; Axelsson et al. 2012; Preece et al. 2014; Ahlgren et al. 2015; Arimoto et al. 2016; Burgess et al. 2016; Moretti \& Axelsson 2016), helped place constraints on the Lorentz invariance of the speed of light (Abdo et al. 2009b, 2009c; Shao et al. 2010; Nemiroff et al. 2012; Vasileiou et al. 2013) and the gamma-ray opacity of the universe (Abdo et al. 2010b; Desai et al. 2017), and motivated revisions in our basic understanding of collisionless relativistic shock physics (Ackermann et al. 2014).

The first Fermi-LAT GRB catalog was published in 2013 (Ackermann et al. 2013b, hereafter 1FLGC). It is a compilation of the 35 GRBs, including 30 long-duration ( $>2 \mathrm{~s}$ ) and 5 shortduration $(<2$ s) GRBs, detected in the period 2008 August

\footnotetext{
${ }^{61}$ Funded by contract FIRB-2012-RBFR12PM1F from the Italian Ministry of Education, University and Research (MIUR).
}

through 2011 July (the first GRB included is GRB $080825 \mathrm{C}$, and the last is GRB 110731A). Of these GRBs, 28 were detected with standard analysis techniques at energies above $100 \mathrm{MeV}$, while 7 GRBs were detected at only $<100 \mathrm{MeV}$ using the LAT Low-Energy (LLE) technique. It established three main observational characteristics of the high-energy emission:

1. Additional spectral components: Many of the bright GRBs observed by Fermi-LAT were not well fit with a single Band function (Band et al. 1993). In particular, an additional power-law (PL) component was required to account for the high-energy data of four bursts.

2. Delayed onset: The emission above $100 \mathrm{MeV}$ was systematically delayed compared with the emission seen at lower energies, in the $\mathrm{keV}-\mathrm{MeV}$ energy range. Delays of up to $40 \mathrm{~s}$ have been detected, with a few seconds being a typical value.

3. Extended duration: The emission above $100 \mathrm{MeV}$ also systematically lasted longer than the $\mathrm{keV}-\mathrm{MeV}$ prompt emission. The flux generally followed a PL decay with time, $F \propto t^{-\alpha}$, with $\alpha$ close to -1 .

The 1FLGC also left some open questions regarding GRB properties at high energy, which we plan to address in the 2FLGC:

1. Hyperfluent GRBs: Four GRBs of the 1FLGC hinted at the possibility of a different class of high-energy fluence, significantly greater than the average fluence of the other GRBs. Because of the small sample, the hyperfluent class of GRBs was not significant and its confirmation was left for subsequent observations. 
2. Late-time temporal decay index: The distribution of the late-time temporal decay index in the 1FLGC was clustered around -1 , supporting the hypothesis of an adiabatically expanding fireball as a common origin of the extended emission. Although this scenario could explain all observations, only nine GRBs had enough data to allow the decay index to be determined.

3. Breaks in the late-time light curve: Three GRBs in the 1FLGC showed breaks in the temporal decay at late times, similar to the breaks observed in the X-ray light curves. Exploration of this feature was limited by the small sample and the relatively low significance of the breaks.

Since the publication of the 1FLGC, a number of improvements have been made with regard to LAT data processing. The two major changes concern the development of a new event analysis. Since launch, the LAT event classes have undergone a number of versions (or "passes"), and the latest "Pass 8" analysis constitutes a major improvement on the previous versions. In addition, a new detection algorithm has been developed running in parallel over a range of different timescales. Taken together, this has increased the detection efficiency by over $60 \%$ (Vianello et al. 2015), and in particular allows the detection of fainter high-energy GRB counterparts.

This paper presents the second catalog of GRBs detected by the Fermi-LAT (2FLGC), covering a $10 \mathrm{yr}$ period, from 2008 August to 2018 August. During this time, the GBM triggered on 2357 GRBs, approximately half of which were in the field of view (FOV) of the LAT at the time of trigger. LAT counterparts are searched for in ground processing, following external triggers provided by the GBM, as well as by other instruments. The LAT instrument is also capable of detecting GRBs through an onboard trigger search algorithm. This is a very rare occurrence and has so far only happened 4 times (see Section 4). In addition, continual blind searches are performed as part of the standard ground processing, to look for untriggered events. These efforts are further described in Ackermann et al. (2016) and Ajello et al. (2018).

In the 2FLGC, we have performed an entirely new, standardized analysis to look for LAT counterparts to all GRB triggers reported during the first decade of Fermi operations. In Section 2 we describe the data used in this study, giving first a short description of the Fermi instruments, followed by the description of data cuts and sample selection. This is followed in Section 3 by a detailed description of the analysis methods used for detection and localization. We also present the methodology that we followed to characterize the temporal and spectral properties of the detected GRBs. The 2FLGC is focused on the LAT-only properties of the bursts in our sample, and we do not perform any joint spectral analysis of LAT and GBM data. In Sections 4 and 5 we present and discuss our results. Finally, we examine the theoretical implications of our results in Section 6.

\section{Data Preparation}

The 2FLGC presents analysis done with data from the LAT, i.e., covering energies above $30 \mathrm{MeV}$ for $\mathrm{GRBs}$ detected through LLE, and from $100 \mathrm{MeV}$ to $300 \mathrm{GeV}$ for GRBs detected with the standard analysis. However, the GBM provides the vast majority of the GRB triggers and is an integral part of the GRB observations made by Fermi. We therefore begin with a brief description of both instruments. This is followed by a more thorough description of the LAT data used for the analysis. Finally, we present the selection of GRB triggers that formed the seed of the catalog.

\subsection{Instrument Overview}

The LAT is a pair production telescope sensitive to $\gamma$-rays in the energy range from $\sim 30 \mathrm{MeV}$ to more than $300 \mathrm{GeV}$. The instrument and its on-orbit calibrations are described in detail in Atwood et al. (2009) and Abdo et al. (2009d). The telescope consists of a $4 \times 4$ array of identical towers, each including a tracker of $18 x-y$ silicon strip detector planes interleaved with tungsten foils, followed by an 8.6 radiation length imaging calorimeter made with $\mathrm{CsI}(\mathrm{Tl})$ scintillation crystals with a hodoscopic layout. This array is surrounded by a segmented anticoincidence detector made of 89 plastic scintillator tiles that identifies and rejects charged particle background events with an efficiency above $99.97 \%$ (Ackermann et al. 2012a).

Whether or not an event is observable by the LAT is primarily defined by two angles: the angle $\zeta$ with respect to the spacecraft zenith, and the viewing angle $\theta$ from the LAT boresight. The LAT performance-including the dependence of the effective area on energy and $\theta$-is presented on the official Fermi-LAT performance web page. ${ }^{62}$ In the analysis performed in this catalog, we do not make any explicit cuts on the angle $\theta$; however, the exposure will drop very quickly for $\theta$ greater than $\sim 75^{\circ}$. The wide FOV $(\sim 2.4 \mathrm{sr}$ at $1 \mathrm{GeV}$ ) of the LAT, its high observing efficiency (obtained by keeping the FOV on the sky with scanning observations), its broad energy range, its large effective area, its low dead time per event $(\sim 27 \mu \mathrm{s})$, its efficient background rejection, and its good angular resolution (the $68 \%$ containment angle of the point-spread function is 0.8 at $1 \mathrm{GeV}$ ) are vastly improved in comparison with those of previous instruments such as EGRET (Esposito et al. 1999). As a result, the LAT provides more GRB detections, higher statistics per detection, and more accurate localizations.

The GBM is composed of 12 sodium iodide (NaI) and two bismuth germanate (BGO) detectors sensitive in the $8 \mathrm{keV}-$ $1 \mathrm{MeV}$ and $250 \mathrm{keV}-40 \mathrm{MeV}$ energy ranges, respectively. The $\mathrm{NaI}$ detectors are arranged in groups of three at each of the four edges of the spacecraft, and the two BGO detectors are placed symmetrically on opposite sides of the spacecraft, resulting in an FOV of 9.5 sr. Triggering and localization are determined from the NaI detectors, while spectroscopy is performed using both the NaI and BGO detectors. The GBM flight software continually monitors the detector rates and triggers when a statistically significant rate increase occurs in two or more $\mathrm{NaI}$ detectors. A combination of 28 timescales and energy ranges are currently tested, with the first combination that exceeds the predefined threshold (generally $4.5 \sigma$ ) being considered the triggering timescale. Localization is performed using the relative event rates of detectors with different orientations with respect to the source and is typically accurate to a few degrees (statistical uncertainty). An additional systematic uncertainty has been characterized as a core-plus-tail model, with $90 \%$ of GRBs having a $3.7^{\circ}$ uncertainty and a small tail suffering a larger than $10^{\circ}$ systematic uncertainty (Connaughton et al. 2015). The GBM covers roughly four decades in energy and provides a bridge from the low energies (below $\sim 1 \mathrm{MeV}$ ),

\footnotetext{
${ }^{62}$ http://www.slac.stanford.edu/exp/glast/groups/canda/lat_Performance.htm
} 
where most of the GRB emission takes place, to the less studied energy range that is accessible to the LAT.

For GRBs exceeding a preset threshold for peak flux or fluence in the GBM, an autonomous repoint request (ARR) is sent to the spacecraft flight software. If the GBM request is accepted, a special LAT observation mode is initiated. This will keep the GBM flight software location in the LAT FOV for an extended period of time, typically $\sim 2.5 \mathrm{hr}$, subject to observational constraints.

On 2018 March 16, one of the solar array drive assemblies on Fermi suffered a malfunction. This led to the LAT and GBM being switched off, and normal science operations were only resumed on April 13. However, as of writing one of the solar panels remains in a fixed position, and a modified rocking strategy has been adopted. As a result, the LAT rocks between the northern and southern sky every week, as opposed to every orbit as before. A further impact is that ARRs have been disabled.

\subsection{Sample Selection}

The 2FLGC presents the results of a search for high-energy counterparts of GRBs that triggered space instruments and have an available public localization. We considered in particular bursts detected by the GBM, the Swift Burst Alert Telescope (BAT; Barthelmy et al. 2005) on board the Neil Gehrels Swift Observatory (Gehrels et al. 2004), and the International Gamma-Ray Astrophysics Laboratory Soft Gamma-Ray Imager (INTEGRAL/ISGRI; Lebrun et al. 2003) on board the INTEGRAL satellite (Winkler et al. 2003), or reported by the Interplanetary Network (IPN ${ }^{63}$ ). In the 10 yr period covered by the 2FLGC, there were 2357 GBM GRB triggers, 876 Swift/ BAT GRB triggers, 65 INTEGRAL/ISGRI GRB triggers, and 83 events reported by the IPN (a few through private communication by the PI K. Hurley). We also considered seven bursts contained in the first catalog of GRBs detected by the Astrorivelatore Gamma a Immagini Leggero minicalorimeter (AGILE/MCAL; Galli et al. 2013) and not contained in any other list. After accounting for bursts that triggered more than one instrument, we have a total of 3044 independent GRBs. We use the localizations provided by the GBM, unless a better localization is provided by one of the instruments on board Swift, namely, the BAT, the X-ray Telescope (XRT; Burrows et al. 2005), or the UV-Optical Telescope (UVOT; Roming et al. 2005), or by the IPN. All these latter positions are distributed via the Gamma-Ray Burst Coordinates Network (GCN) ${ }^{64}$ while the GBM-only localizations are reported in the Fermi-GBM online GRB Catalog ${ }^{65}$ (hereafter FGGC; see Bhat et al. 2016, for more details).

\subsection{LAT Data Cuts and Temporal Selection}

For the standard analysis, we use Pass 8 LAT data with energies between $100 \mathrm{MeV}$ and $100 \mathrm{GeV}$, selecting the time interval around each trigger from $600 \mathrm{~s}$ before to $100 \mathrm{ks}$ after the GRB trigger time, and defining a standard region of interest (ROI) around the trigger location of $12^{\circ}$. In order to reduce the contamination of the Earth limb, in some dedicated cases (13 GRBs) we define a smaller ROI with a radius of $8^{\circ}$. It is worth

\footnotetext{
63 http://www.ssl.berkeley.edu/ipn3/

${ }^{64}$ https://gcn.gsfc.nasa.gov/gcn3_archive.html

${ }^{65}$ https://heasarc.gsfc.nasa.gov/W3Browse/fermi/fermigbrst.html
}

noting that as a final check we look for high-energy events over a larger energy range (up to $300 \mathrm{GeV}$ ), as discussed in Section 4.8.

We then use gtmktime to select only those time intervals when the center of the ROI has a zenith angle $\zeta<97^{\circ}$ (i.e., every point of the ROI has $\zeta<105^{\circ}$ ). For bursts with an initial value of $\zeta>90^{\circ}$, we increase our selection to include all time intervals when the center of the ROI has a zenith angle $\zeta<102^{\circ}$. This allows us to study the prompt emission of those GRBs that started close to the limb of the Earth. The choice of the event class depends on the timescale on which we detect the signal from the GRB and is described in Section 3.2.

\subsection{LLE Data}

The LLE technique is an analysis method designed to study bright transient phenomena, such as GRBs and solar flares, in the $30 \mathrm{MeV}-1 \mathrm{GeV}$ energy range. The LAT Collaboration developed this analysis using a different approach than the one used in the standard photon analysis, which is based on sophisticated classification procedures (a detailed description of the standard analysis can be found in Atwood et al. 2009; Ackermann et al. 2012a). The idea behind LLE is to maximize the effective area below $\sim 1 \mathrm{GeV}$ by relaxing the standard analysis requirement on background rejection.

The basic LLE selection is based on a few simple requirements on the event topology in the three LAT subdetectors. First of all, an event passing the LLE selection must have at least one reconstructed track in the tracker and therefore an estimate of the direction of the incoming photon. Second, we require that the reconstructed energy of the event be nonzero.

We use the information provided by the flight software in LLE to efficiently select events that are gamma-ray like. With the release of Pass 8 data, we have also improved the LLE selection. For events with an incident angle $\theta<40^{\circ}$, we require that no anticoincidence tiles are in "veto" condition (to suppress charged particle contamination), while for angles greater than $40^{\circ}$, we allow a maximum of two tiles in "veto" condition, but no tracker hits can be found in proximity of the anticoincidence hits. This condition helps prevent suppression of large incident angle gamma-rays due to secondary electrons or positrons interacting with the anticoincidence detector downstream. In order to reduce the number of photons originating from the Earth limb in our LLE sample, we only keep reconstructed events with a zenith angle $\zeta<90^{\circ}$ or $\zeta<100^{\circ}$ (depending on the location of the GRB). Finally, we explicitly include in the selection a cut on the ROI, i.e., the position in the sky of the transient source we are observing. In other words, the localization of the source is embedded in the event selection, and therefore for a given analysis the LLE data are tailored to a particular location in the sky. The response of the detector for the LLE class is encoded in a response matrix, which is generated using a dedicated Monte Carlo simulation for each GRB, and is saved in the standard HEASARC RMF file format. ${ }^{66}$ LLE data and the relative response are made available for any transient signal (GRB or solar flare) detected with a significance above $4 \sigma$ through the HEASARC FERMILLE website. $^{67}$

\footnotetext{
${ }^{66}$ Described at http://heasarc.gsfc.nasa.gov/docs/heasarc/caldb/docs/memos/ cal_gen_92_002/cal_gen_92_002.html\#Sec:RMF-format.

67 http://heasarc.gsfc.nasa.gov/W3Browse/fermi/fermille.html
} 


\subsection{Low-energy Data Used for Comparisons}

While we do not perform any joint spectral fitting with GBM data in this work, comparisons with the sample of GBMdetected GRBs are both highly interesting and inevitable in order to characterize our sample. For this purpose we use the official data from the FGGC.

In order to perform the comparisons, we use the standard GRB properties that characterize the GRB emission, in particular the onset time and duration (both calculated by GBM in the 50-300 keV energy range), values of peak fluxes $\left(F_{P}\right.$, calculated in $1024 \mathrm{~ms}$ and $64 \mathrm{~ms}$ intervals for long and short GRBs, respectively), fluences ( $F$, calculated over the time interval used in the GBM spectral analysis, which might not always be coincident with the burst duration), and the spectral parameters of the best-fit model derived by the GBM in the $10-1000 \mathrm{keV}$ energy band. The best fit is determined from the four standard spectral models tested in the GBM timeintegrated spectral catalog analysis (see Gruber et al. 2014, for more details), namely, the simple PL, the smoothly broken PL (SBPL), the phenomenological Band function (BAND; Band et al. 1993), and the Comptonized model (COMP), which is a subset of the Band function. The latter three models are characterized by a low-energy spectral index $\alpha$, by a highenergy spectral index $\beta$, which in the case of the COMP model goes to minus infinity, and by the energy $E_{\text {peak }}$, which describes the peak of the $\nu F_{\nu}$ distribution.

The classification of GRBs into long and short classes is primarily derived from the low-energy duration as measured by GBM and follows the standard rule that long and short bursts are longer and shorter than $2 \mathrm{~s}$, respectively (Kouveliotou et al. 1993). For bursts that did not trigger GBM and are not included in the FGGC, we use the durations calculated by the KonusWind instrument in the $20 \mathrm{keV}-5 \mathrm{MeV}$ energy range, which have been published in GCN Circulars.

\section{Analysis Methods and Procedures}

As presented in Section 1, significant improvements have been made to the analysis techniques since the 1FLGC. The two developments with highest impact are the "Pass 8" event analysis and a redesigned detection algorithm. "Pass 8" has been rebuilt from the ground up with respect to previous versions ("Pass 6" was used for the first catalog), resulting in increased sensitivity. It is thoroughly described in Atwood et al. (2013a). In this section, we focus on the improvements to the triggered search and detection algorithms for GRBs used to produce the 2 FLGC.

\subsection{Analysis Sequence}

The procedure followed to produce the catalog is summarized in Figure 1. Each individual step of the analysis is described in detail in the following subsections.

Each input trigger was first run through a detection algorithm as described in Section 3.2. All triggers that passed the criteria for detection in any time window were placed in the list of potential candidates for analysis. This list was manually inspected following the procedure in Section 3.5. Only detections retained after this inspection were passed to the analysis pipeline. Here, analyses were performed to determine a number of key properties for each GRB, such as onset, duration, and spectral parameters. The steps of this analysis are described in Section 3.6.

\subsection{The LAT Transient Factory}

The LAT Transient Factory (LTF) algorithm was introduced after the publication of the 1FLGC and is presented in Vianello et al. (2015). LTF has been running continuously ever since, looking in real time for GRB counterparts in LAT data. When compared to the old algorithm on the same data set as the first catalog, it returned 50\% more GRBs. The adoption of "Pass 8" data has led to a further $10 \%$ improvement.

LTF is based on the application of an unbinned maximum likelihood technique. This analysis starts by selecting all the photons detected by the LAT above $100 \mathrm{MeV}$ in a circular ROI with radius $r$ and center $\left(\alpha_{\mathrm{ROI}}, \delta_{\mathrm{ROI}}\right)$ in a time window starting at the trigger time $T_{0}$. Then, the presence of a new point source at a position $\left(\alpha_{t}, \delta_{t}\right)$ is tested by using the likelihood-ratio test (LRT). The null hypothesis for the test is represented by a baseline likelihood model including all point sources from the LAT source catalog (Acero et al. 2015) with all parameters fixed, as well as the Galactic and isotropic diffuse emission templates ${ }^{68}$ provided by the Fermi-LAT Collaboration (Acero et al. 2016), with the normalizations left free to vary. The alternative hypothesis is represented by the baseline model plus the new point source ("test source"), modeled with a PL spectrum with free index and normalization. The LRT uses as the test statistic (TS) twice the logarithm of the maximum of the likelihood function for the alternative hypothesis $\left(L_{1}\right)$ divided by the maximum of the likelihood function for the null hypothesis $\left(L_{0}\right)$ :

$$
\mathrm{TS}=2\left(\log L_{1}-\log L_{0}\right) .
$$

Detailed instructions on how to perform an unbinned likelihood analysis using the Fermi Science Tools can be found on the Fermi website. ${ }^{69}$

For each trigger, a search is performed on five time windows starting at the trigger time $T_{0}$ and ending 10, 100, 500, 4000, and $10,000 \mathrm{~s}$ after $T_{0}$. This selection slightly differs from the standard LTF real-time analysis, which consists of 10 searches running in parallel over time intervals logarithmically spaced from the trigger time to $10 \mathrm{ks}$ after that, as stated in Vianello et al. (2015). For the 10 and $100 \mathrm{~s}$ time windows, we use the Pass 8 P8R2_TRANSIENT020E_V6 event class and the corresponding response functions; for the longer time windows, the event class P8R2_TRANSIENT010E_V6 is used. In each time window the LTF starts from the input coordinates and trigger time as measured by the triggering instrument (see Section 2.2) and performs the following steps:

1. Finding map: we consider an ROI with radius $r$ centered on the input position and a square grid of side $\delta x$ inscribed in the ROI with a spacing of 0.8 . The size of the grid is fixed according to the triggering instrument and its typical localization accuracy (statistical + systematic), as well as the typical size of the LAT PSF. Specifically, $\delta x=32^{\circ}$ for triggers localized by the GBM that are dominated by systematic uncertainties (Connaughton et al. 2015), $\delta x=1.6$ for Swift and INTEGRAL triggers, and $\delta x=5^{\circ}$ for IPN triggers. The radius of the $\mathrm{ROI}$ is chosen as $r=\delta x / 2+12^{\circ}$ in order to have enough data around each point in the grid for performing an LRT

\footnotetext{
68 gll_iem_v06.fits and iso_P8R3_SOURCE_V2.txt; https://fermi gsfc.nasa.gov/ssc/data/access/lat/BackgroundModels.html.

69 https://fermi.gsfc.nasa.gov/ssc/data/analysis/scitools/likelihood_tutorial.html
} 


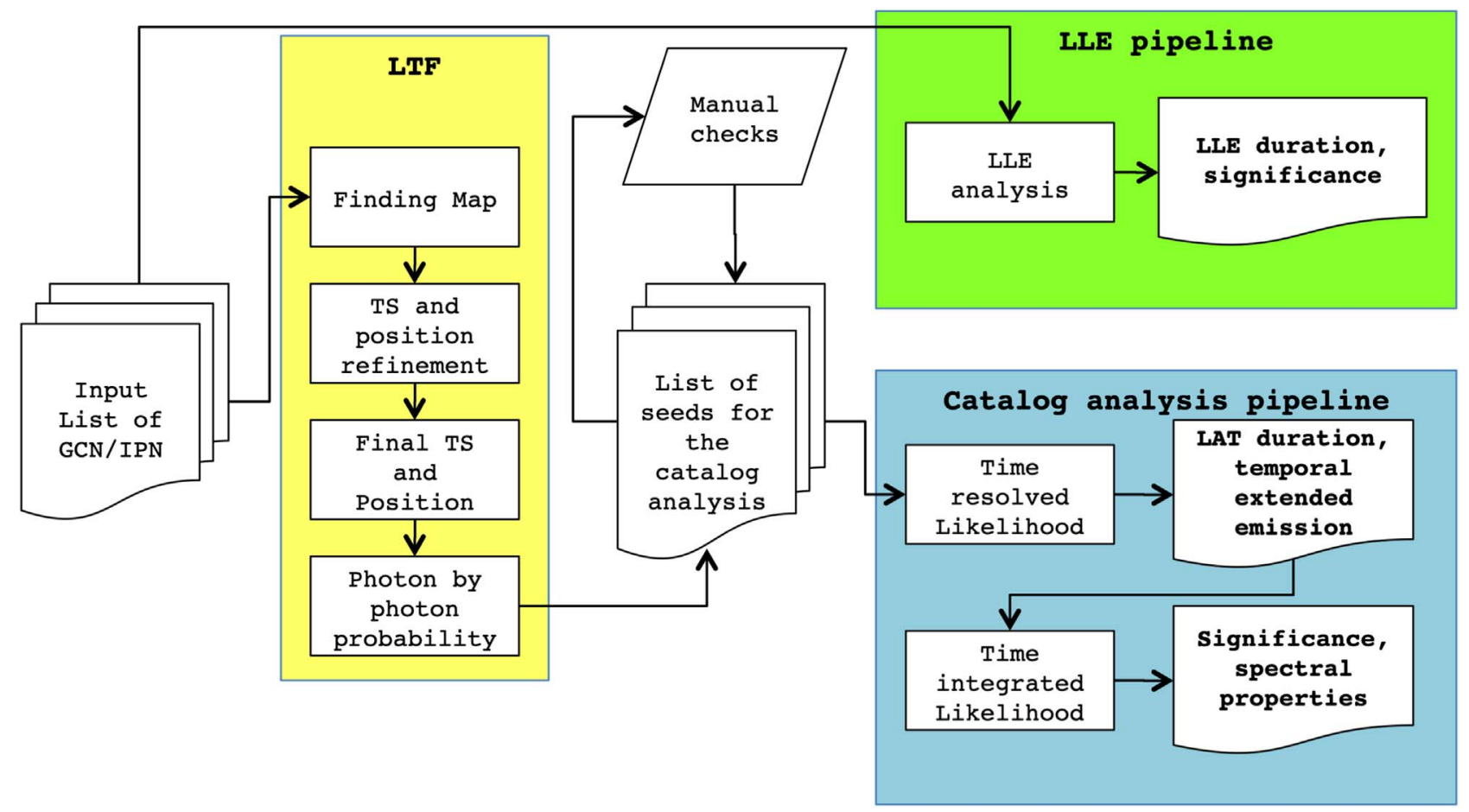

Figure 1. Flow diagram representation of the analysis pipeline. The LTF detection algorithm is highlighted in yellow, and the catalog analysis pipeline, which is executed after manually checking the candidate seeds, is highlighted in blue. The LLE analysis pipeline, which is executed on all input triggers, is highlighted in green. All input candidates are analyzed by the LTF/LLE pipelines; only those passing the detection criteria are retained. Inspection in the manual checks can also lead to rejection of further cases (see text for details).

test (see below). In order to reduce the contamination from the Earth limb-a bright source of $\gamma$-rays-all events with $\zeta<100^{\circ}$ are filtered out. The effect of this selection is taken into account when computing the exposure by the tool gtltcube. We then use the LRT as described above to test for the presence of a source at each position of the grid having at least three photons within $10^{\circ}$. The latter requirement filters out points without any photon cluster around them, in order to reduce the computational cost. The point $\left(\alpha_{\max }, \delta_{\max }\right)$ in the grid providing the maximum of the TS is considered the best guess for the position of the new transient and marked for further analysis.

2. TS and position refinement: we consider an ROI centered on $\left(\alpha_{\max }, \delta_{\max }\right)$ with a radius of $8^{\circ}$, and we perform an LRT as described above considering only the time intervals within the time window when the border of the ROI is at a zenith angle smaller than $105^{\circ}$ ("good time intervals"). This is a different way of reducing the contamination from the Earth limb that is more effective than the one used in the previous step, but it can only be applied on small ROIs. We then use the tool gtfindsrc to search for the maximum of the likelihood under the alternative hypothesis (i.e., when the test source is added to the model), varying the position of the test source and profiling the other free parameters. The position $\left(\alpha_{1}, \delta_{1}\right)$ yielding the maximum of the likelihood is considered the new putative position for the candidate counterpart.

3. Final TS and position: the previous step is repeated using an ROI centered on $\left(\alpha_{1}, \delta_{1}\right)$ that yields the final TS $\left(\mathrm{TS}_{\mathrm{GRB}}\right)$. The tool gtfindsrc is run again returning the final estimate of the localization uncertainty.
4. Photon-by-photon assignment of probability: we run the tool gtsrcprob using the final optimized likelihood model under the alternative hypothesis. This tool assigns to each detected photon the probability of belonging to the test source, i.e., to the candidate counterpart. We then measure the number of photons $N_{p}>0.9$ having a probability larger than $90 \%$ of belonging to the candidate counterpart.

The final products of LTF are five sets of results, one for each time window. In order to consider a counterpart detected, we consider in particular $\mathrm{TS}_{\mathrm{GRB}}$ and $N_{p>0.9}$, as explained in the next section.

\subsection{Detection Threshold and False Discovery Rate}

A classic result from Chernoff (1954) states that under the null hypothesis the TS of a single LRT as applied in LTF is a random variable that is zero half of the time and is distributed as $\chi^{2}$ with 1 degree of freedom the other half. This result was confirmed by Monte Carlo simulation in Mattox et al. (1996). Under these circumstances, the significance of the detection (z-score) is $\sqrt{\mathrm{TS}}$; thus, a threshold of TS $=25$ corresponds to a detection $>5 \sigma$ for one LRT.

As described in the previous section, LTF consists of multiple LRT procedures and the trial factor needs to be taken into account. The effective number of trials for one time window is, however, difficult to determine because the trials are not independent. Furthermore, we also need to account for the number of time windows and for the number of triggers searched.

To account for the number of triggers searched, we use the procedure proposed by Benjamini \& Hochberg (1995). It assumes independent trials and is simple: all the $p$-values $p_{i}$ for 
all the $n_{\mathrm{tr}}$ searches, with $i=1 \ldots n_{\mathrm{tr}}$, are sorted in increasing order. We then find $k$ so that $p_{k}$ is the largest $p$-value where $p_{k} \leqslant\left(k / n_{\text {tr }}\right) \alpha$, where $\alpha$ is the error probability for one test. All the triggers with $i=1 \ldots k$ are considered detected. In practice, we first compute through Monte Carlo simulations the effective number of trials for one time window $n_{\text {trw }}$. The value of $n_{\text {trw }}$ is different depending on the instrument that generated the trigger, and it reflects the size of the finding map (see previous section), so it is larger for larger finding maps. We find $n_{\text {trw }}=110$ for GBM triggers, $n_{\text {trw }}=12.28$ for IPN triggers, and $n_{\text {trw }}=1.25$ for Swift and INTEGRAL triggers. We then compute the post-trial $p$-value for one time window by using the binomial distribution as $p_{\mathrm{w}}=1-\left(1-p_{\mathrm{pr}}\right)^{n_{\mathrm{trw}}}$, where $p_{\mathrm{pr}}$ is the $p$-value coming from the LRT applied to the current time window. There is also another independent trial factor $n_{\mathrm{et}}$, which we consider to be equal to the number of time windows where we effectively searched for a counterpart. Note that this is a slightly conservative approach, as the time windows are not independent and thus $n_{\mathrm{et}}$ is in reality a little smaller. The number $n_{\mathrm{et}}$ can vary from 0 to 5 depending on how many time windows had an exposure larger than zero after our data cuts. For example, if the trigger was never in the FOV or it was always at a zenith angle larger than our cut during a time window, this will not constitute a search, and it will not contribute to $n_{\text {et }}$. We can now compute the $p$-value for a GRB corrected for both the spatial and the time trials as $p=1-\left(1-p_{\mathrm{w}, \min }\right)^{n_{\mathrm{et}}}$, where $p_{\mathrm{w}, \min }$ is the minimum $p_{\mathrm{w}}$ corresponding to the maximum final $\mathrm{TS}_{\mathrm{GRB}}$ found by $\mathrm{LTF}$ among the timescales searched. We then apply the Benjamini $\&$ Hochberg (1995) procedure using these $p$-values and correct for the number of triggers searched as explained above.

We further apply the quality cut $n_{\mathrm{p}>0.9} \geqslant 3$ to the list of detections, i.e., we require at least three photons with a probability larger than $90 \%$ of belonging to the GRB. This neutralizes the effect of isolated high-energy photons $(\gtrsim 10 \mathrm{GeV})$ within the search region that tend to return high TS values during the unbinned analysis but also very hard spectra, with photon indices close to 0 . Moreover, three photons are required in order to have both the normalization and the photon index free during the likelihood maximization under the alternative hypothesis and still have at least 1 degree of freedom.

\subsection{The Bayesian Blocks Burst Detection Algorithm for LLE Data}

In order to detect GRB counterparts in LLE data, we use a counting analysis based on the well-known Bayesian blocks (BB) algorithm of Scargle et al. (2013). The BB algorithm is capable of dividing a time series into intervals of constant rate, opening a new block only when the rate of events changes in a statistically significant way. In particular, we use the unbinned version of the algorithm, which presents as the only parameter the probability $p_{0}$ of opening a new block when the rate is constant (false positive). However, before we can apply the BB algorithm, we need to introduce a preprocessing step to account for the time-varying background in LLE data. Otherwise, the $\mathrm{BB}$ algorithm will find many blocks following the variation in the event rate owing to the variations in the background.

We start by fitting a polynomial function to the data in two off-pulse time windows, one before and one after the trigger window, as shown in the left panel of Figure 2 for GRB 131014A. The trigger window is defined on the basis of the duration measured by the GBM in the $50-300 \mathrm{keV}$ energy range. Then, we exploit the fact that a nonuniform Poisson process with expected value $\lambda(t)$ can be converted to a uniform one by transforming the time reference system to

$$
t^{\prime}=\int_{0}^{t} \lambda(t) d t
$$

In our case $\lambda(t)$ is the expected number of events of the timevarying background as modeled by the polynomial. We then transpose the time series of LLE events to the $t^{\prime}$ time reference system and apply the standard BB algorithm. If more than one block is found in the trigger window, it means that there is a rate change on top of what is predicted by the background model. We then deem the transient detected, and we transform back to the original time reference system to yield the time interval of the detection. For all searches we use $p_{0}=10^{-3}$. The analysis is illustrated in the right panel of Figure 2, which shows the final $\mathrm{BB}$ representation of the light curve for GRB 131014A.

\subsection{Final Manual Checks}

The normalization of the Galactic and diffuse templates is allowed to vary in the analysis, but the parameters for the point sources are kept fixed to the values found in the updated FermiLAT point-source list. ${ }^{70}$ This means that the algorithm can potentially pick up non-GRB sources, such as AGN flares. Moreover, GRBs detected far from zenith might also suffer from Earth limb contamination. Therefore, a final manual check of all potential detections was also performed. The list of detections derived by the LTF pipeline previously described was divided into random subsets of bursts that were assigned for analysis to the members of the Fermi-LAT GRB team. Each putative event was independently cross-checked by two people, whose task was to either confirm or reject the detection. In case of agreement, the classification was seen as final; otherwise, the case was reviewed by a third person.

The manual checks included a series of tasks to be carried out. First, the LTF results were evaluated in each of the five temporal intervals, taking into account (1) the number of photons with probability $>90 \%$, (2) the distance to the nearest known source, (3) the localization error, (4) the spectral index, and (5) the final TS value. We identified many "simple" cases, in which both the number of detected photons was high and the final TS was above 80 in several time intervals, and no other known sources were present in the ROI. These candidates were marked as confirmed with no further inspection.

Intermediate cases that needed deeper investigations included (1) cases where the final TS in all time intervals was close to the threshold, (2) cases where only three to four high-energy photons were detected, (3) cases where a bright source (an AGN, solar flares, etc.) was at an angle $<1^{\circ}$ from the GRB candidate in the ROI, and (4) cases where a high TS value was obtained only by integrating over the longest timescale (from 0 to 10,000 s; see Section 3.2). In order to check for other active sources in the ROI around the time of the GRB trigger, we looked for flaring blazars using the publicly available FAVA tool ${ }^{71}$ (Ciprini et al. 2013; Abdollahi et al. 2017), and for solar activity we checked the Solar Monitor public pages. ${ }^{72}$

\footnotetext{
70 https://fermi.gsfc.nasa.gov/ssc/data/access/lat/fl8y/

71 https://fermi.gsfc.nasa.gov/ssc/data/access/lat/FAVA/

72 http://www.solarmonitor.org
} 

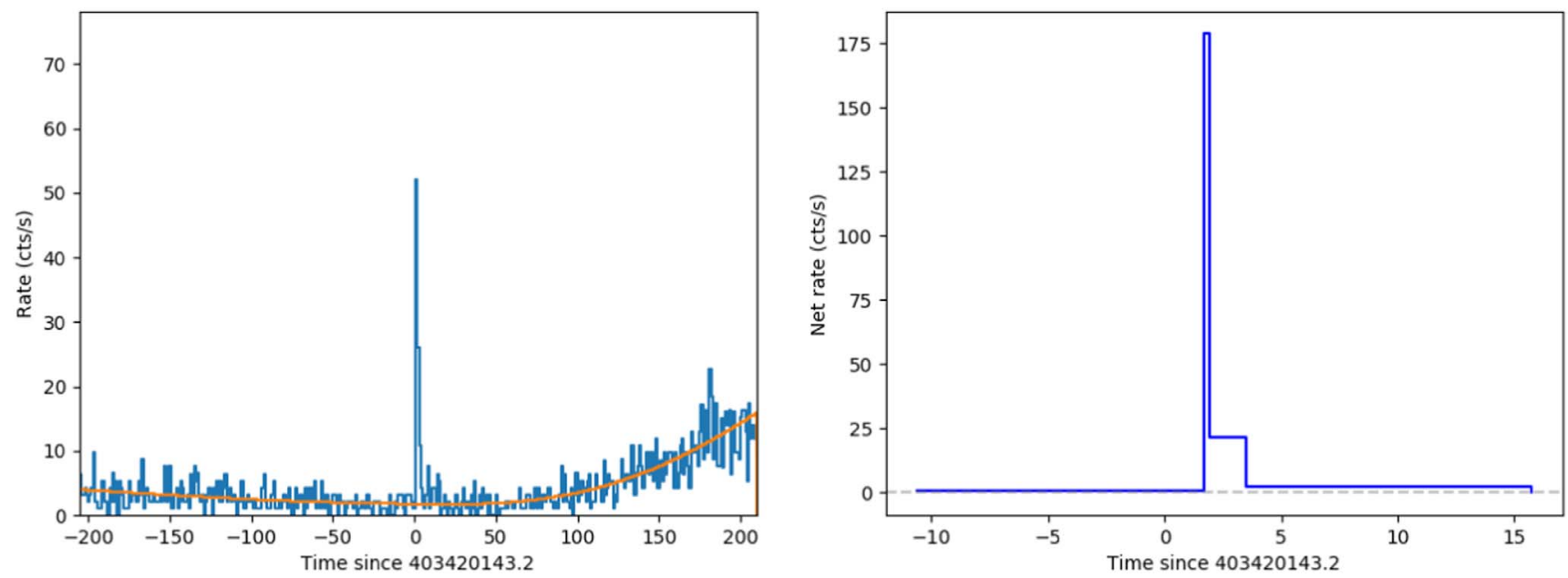

Figure 2. Left panel: LLE light curve of GRB 131014 in the $30 \mathrm{MeV}-1 \mathrm{GeV}$ energy range. The polynomial fit to the background is marked by a red line. Right panel: BB representation of the light curve of GRB 131014. All light curves are centered around the GBM trigger time $T_{0}=403420143.2$ (in MET).

Table 1

Definition of the Four Time Intervals (with Start and Stop Times) Used in the Time-integrated Spectral Analysis

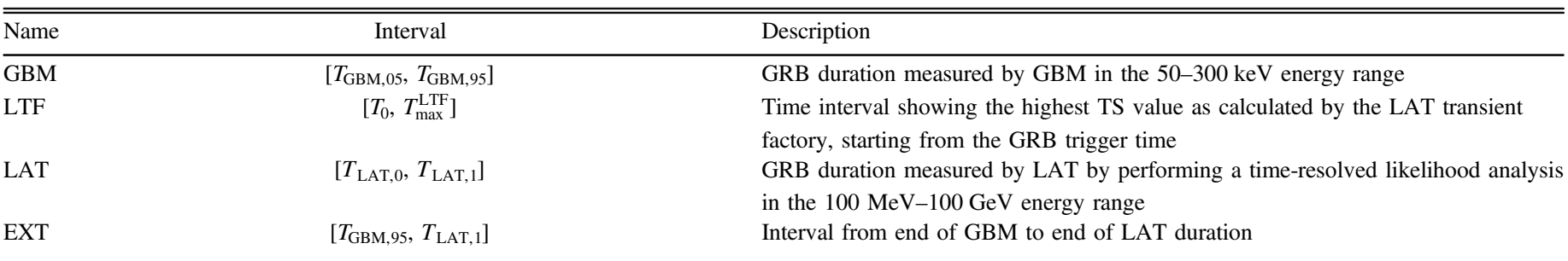

In case of particularly uncertain candidates, we performed an ad hoc likelihood analysis, similar to the one performed by the LTF pipeline, but running on dedicated time intervals that might differ from the catalog ones.

Through these manual checks, $\sim 15 \%-20 \%$ of the examined cases were rejected as not connected to a GRB. The remaining events were processed in the dedicated analysis pipeline.

\subsection{Catalog Analysis Description}

In this section we describe the analysis steps we performed on each GRB of the final sample. The idea is to perform an automated analysis, which is implemented in a series of python scripts that are used to control the various steps. The analysis is based on ScienceTools v11r05p03, available for download at the Fermi Science Support Center. ${ }^{73}$

\subsubsection{Time-integrated Likelihood Analysis}

We perform an unbinned likelihood analysis in four different time intervals. The "GBM" time interval represents the GRB duration as given by $T_{\mathrm{GBM}, 90}$ reported in the FGGC. $T_{\mathrm{GBM}, 90}$ is the interval during which the instrument measures from $5 \%$ to $95 \%$ of the total GRB flux in the $50-300 \mathrm{keV}$ energy range (i.e., from $T_{\mathrm{GBM}, 05}$ to $\left.T_{\mathrm{GBM}, 95}\right)$. The "LTF" interval corresponds to the time interval in the LAT Transient Factory analysis where the highest TS was found. The "LAT" interval encompasses the signal detected by the LAT, as defined in 3.6.2. The "EXT" interval is defined as the time interval including LAT emission (if any) after the $T_{\mathrm{GBM}, 95}$. Table 1 summarizes the definition of these intervals.

\footnotetext{
${ }^{73}$ https://fermi.gsfc.nasa.gov/ssc/data/analysis/
}

\subsubsection{Time-resolved Likelihood Analysis}

In order to perform time-resolved likelihood analysis, we have developed an algorithm for adaptively binning the LAT events. Starting from the result of the analysis in the "LTF" time window, we apply gtsrcprob to calculate the probability of each LAT event to be associated with the GRB source. Starting with preselected logarithmically spaced time bins (48 bins from $0.01 \mathrm{~s}$ to $50 \mathrm{ks}$ after the GBM trigger), we merge them until at least three events with probability $>0.9$ are present in each final bin. In practice, we have 3 degrees of freedom $\left(N_{\mathrm{dof}}\right): 2$ associated with the PL describing the GRB, and 1 with the normalization of the isotropic diffuse component. The normalization of the Galactic model has been fixed to its nominal value (1). We require at least $N_{\text {dof }}$ events with probability $>0.9$ in every bin. In this way, we optimize the duration of the time intervals in order to always have enough photons to perform the fit. Once we have identified the time bins, we perform unbinned likelihood analysis in each bin, calculating the value of flux or, in the case of a TS value $<10$, we calculate the flux upper limit $(95 \%)$ by profiling the likelihood function.

In the 1FLGC, the duration in the LAT was calculated based on the concept of $T_{\mathrm{GBM}, 90}$, i.e., the time during which $90 \%$ of the flux is collected. As the LAT observes each photon individually, this requires the simulation of light curves. In this analysis, we instead use a technique based on the individual photons intrinsic to the LAT. The total duration of the signal in the LAT, defined as $T_{\mathrm{LAT}, 100}$, is estimated starting from the results of the time-resolved analysis. The LAT onset time $T_{\mathrm{LAT}, 0}$ corresponds to the time when the first photon with probability $p>0.9$ to be associated with the GRB is detected, 

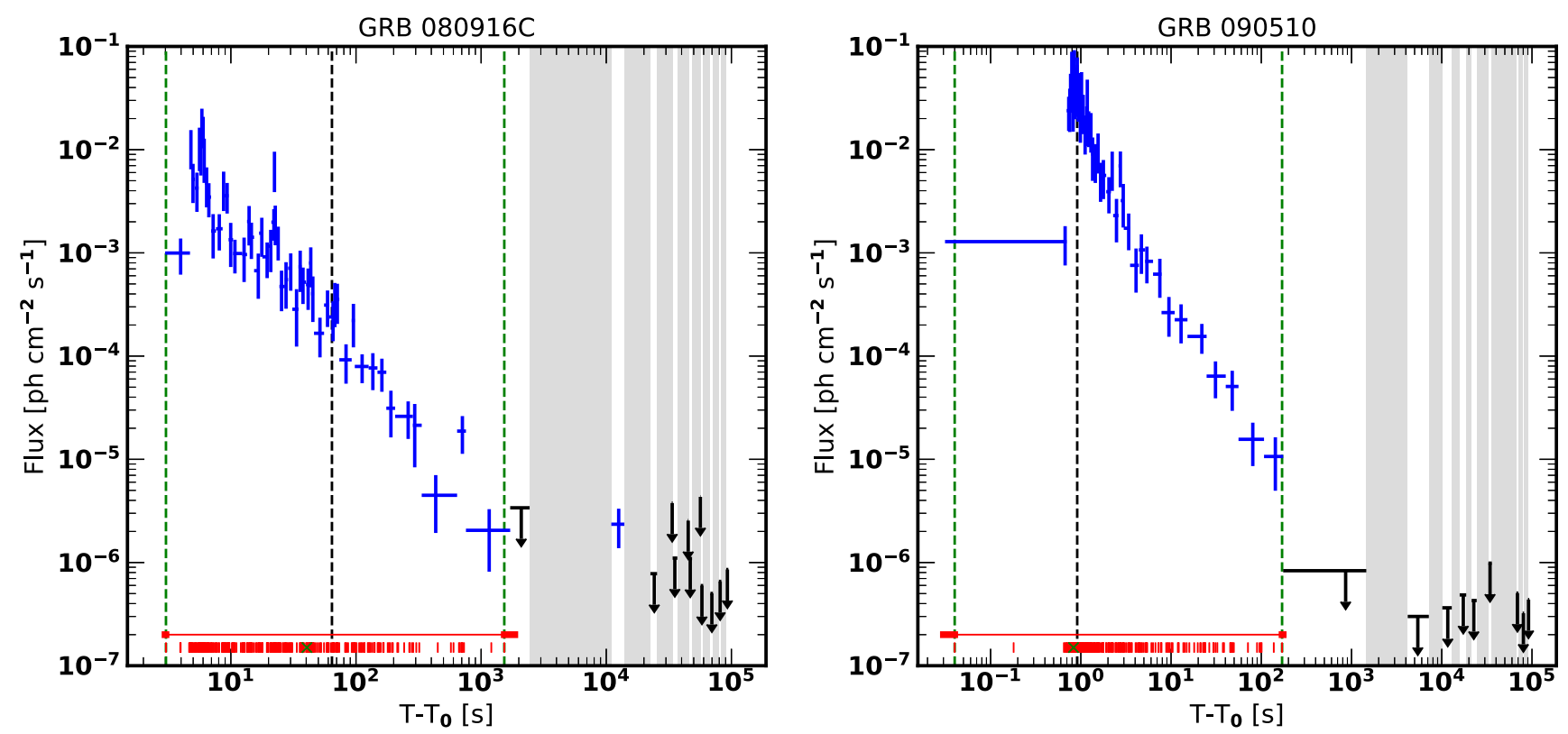

Figure 3. Temporal extended emission for two bright LAT GRBs, the long GRB 080916C (left panel) and the short GRB 090510 (right panel). Blue points show the flux in each time bin, while black arrows mark upper limits. The green vertical dashed lines indicate the first and the last LAT-detected event, while the vertical dashed black line marks the end of the GBM emission $\left(T_{\mathrm{GBM}, 95}\right)$. Shaded gray areas mark intervals when the GRB is outside the FOV. The red vertical markers at the bottom of each panel indicate the arrival times of the events with probability $>0.9$ to be associated with the GRB, with the green cross being the event with maximum energy. The horizontal red line indicates the estimated duration of the GRB $\left(T_{\mathrm{LAT}, 100}\right)$.

while $T_{\mathrm{LAT}, 1}$ corresponds to the last event with $p>0.9$. $T_{\mathrm{LAT}, 100}$ of the signal is simply $T_{\mathrm{LAT}, 1}-T_{\mathrm{LAT}, 0}$. These are also the quantities that define the "LAT" time interval, as previously discussed (see Table 1).

In order to correctly estimate the uncertainty on $T_{\mathrm{LAT}, 1}$ $\left(\delta T_{\mathrm{LAT}, 1}\right)$ for an event with $n$ detected photons with probability $p>0.9$, we define $\Delta t_{n-1, n}$ as the time interval between the second-to-last and the last event. Assuming Poisson statistics, the probability to measure an event between $t$ and $t+d t$ is $P(t$, $t+d t)=\lambda d t$, where $\lambda$ is the rate: in our case $\lambda=2 / \Delta t_{n-1, n}$. Therefore, we conservatively compute the uncertainty as $\delta T_{\mathrm{LAT}, 1}=1 / \lambda=\Delta t_{n-1, n} / 2$. Similarly, considering the first two events with probability $p>0.9$, we define the uncertainty on $T_{\mathrm{LAT}, 0}$ as $\delta T_{\mathrm{LAT}, 0}=\Delta t_{1,2} / 2$. The error on $T_{\mathrm{LAT}, 100}$ follows using standard error propagation.

In order to better illustrate this analysis, Figure 3 shows two light curves of two bright GRBs, the long GRB 080916C (left panel) and the short GRB 090510 (right panel). For the long burst, the arrival time of the last event is substantially smaller than the end of the bin of the last detection. This could indicate that in the last bin the GRB is only marginally detected. For most of the other bursts, the arrival time of the last event is very close to the end of the last bin with positive detection, as in the case of GRB 090510.

\subsubsection{Calculation of Energetics}

In addition to reporting the flux and fluence of each GRB, for the subset of GRBs with measured redshift $z$ we also calculate their total radiated energy $\left(E_{\text {iso }}\right)$. Starting from the measured spectrum of each burst, this is done by using the best-fit model over a specific energy range and by assuming that the energy emitted by a GRB at the source in the cosmological source frame is isotropically radiated. The isotropic radiated energy is defined by following expression:

$$
E_{\text {iso }}=\frac{4 \pi d_{L}^{2}}{1+z} S\left(E_{1}, E_{2}, z\right),
$$

where $d_{L}$ is the luminosity distance and $S\left(E_{1}, E_{2}, z\right)$ is the fluence integrated between the minimum energy $E_{1}$ and the maximum energy $E_{2}$. It can be expressed as

$$
S\left(E_{1}, E_{2}, z\right)=T_{100} \int_{E_{1} /(1+z)}^{E_{2} /(1+z)} E N(E) d E .
$$

Here $N(E)$ describes the best-fit spectral model, and $T_{100}$ represents the total duration of the burst as defined in the previous section. LAT data are always fit with a simple PL model in the energy range $100 \mathrm{MeV}-10 \mathrm{GeV}$, i.e.,

$$
N(E)=A E^{\Gamma} .
$$

Finally, assuming a spatially flat universe $\Lambda \mathrm{CDM}$ model with $\Omega_{\Lambda}=0.714, \quad \Omega_{M}=0.286$, and $H_{0}=69.6 \mathrm{~km} \mathrm{~s}^{-1} \mathrm{Mpc}^{-1}$ (Bennett et al. 2014; Planck Collaboration et al. 2016), the luminosity distance is given by (Weinberg 1972)

$$
d_{L}\left(z, \Omega_{\Lambda}, \Omega_{M}\right)=(1+z) \frac{c}{H_{0}} \int_{0}^{z} \frac{d z^{\prime}}{\sqrt{\Omega_{M}\left(1+z^{\prime}\right)^{3}+\Omega_{\Lambda}}} .
$$

\subsubsection{Localization}

The LTF algorithm described in Section 3.2 returns the position of the GRB, as well as its detection probability. The steps of the procedure include refining the source location, and the position given in the final step is taken as the definitive one; no further optimization is performed. 

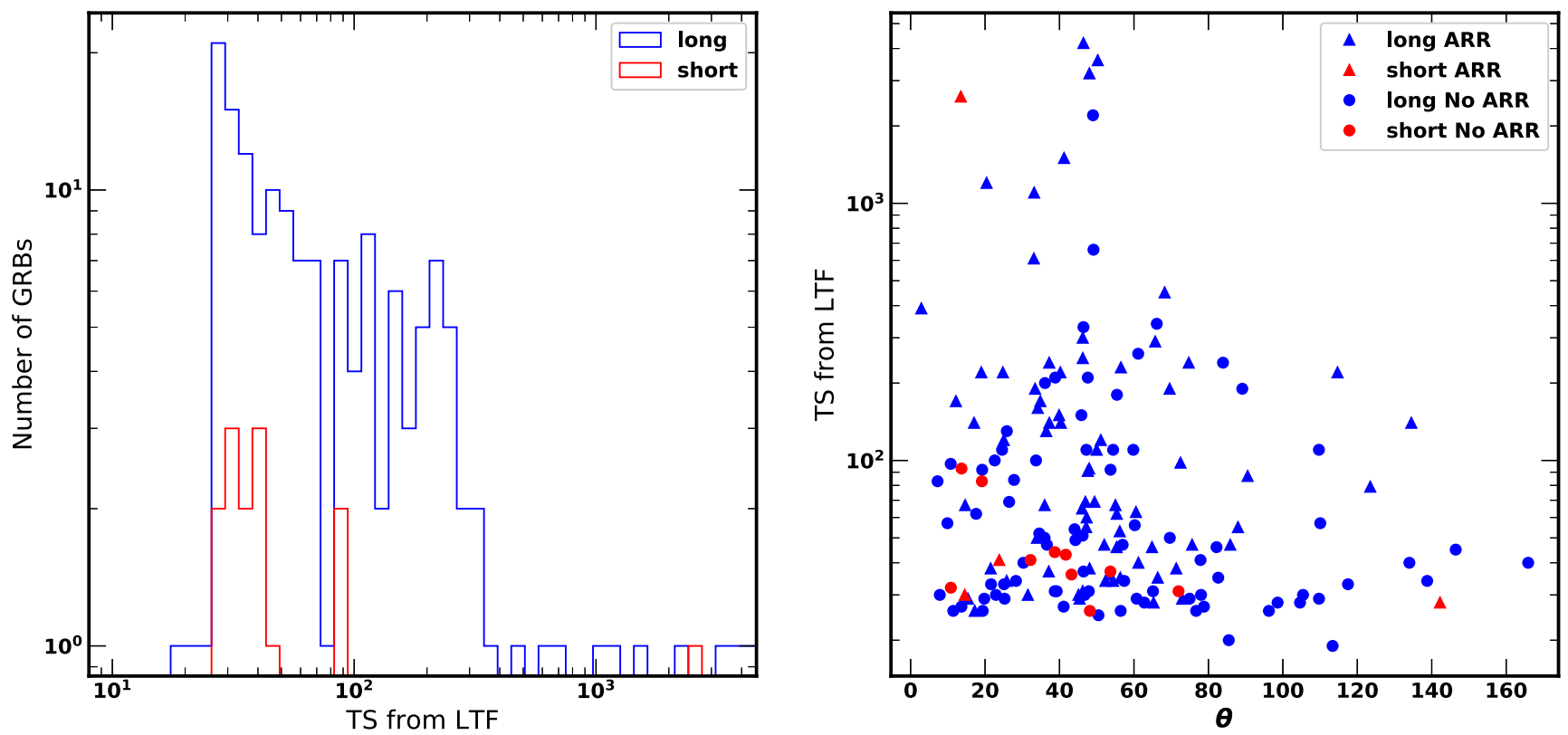

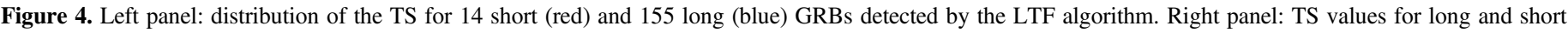
bursts as a function of the angle $\theta$ at the trigger time. Bursts that triggered an ARR are marked with a triangle.

\subsubsection{LLE Light Curve and Duration}

The BB burst detection algorithm described in Section 3.4 provides a way of binning the data taking into account background fluctuations: blocks are defined only when an intrinsic rate variation above the background is detected, as opposed to an absolute variation. In our analysis we therefore define the onset of the LLE signal $\left(T_{\mathrm{LLE}, 05}\right)$ as the starting time of the first block above background. Similarly, we define the $T_{\mathrm{LLE}, 95}$ as the ending time of the last block above background. The LLE duration $\left(T_{\mathrm{LLE}, 90}\right)$ is simply defined as $T_{\mathrm{LLE}, 95}-T_{\mathrm{LLE}, 05}$.

\section{Results}

In the following subsections, we examine the main results of our analysis. The focus will be on the properties of the overall population, rather than a presentation of individual GRBs.

\subsection{LAT Detections}

This $10 \mathrm{yr}$ catalog comprises 186 detections, 17 short GRBs (sGRBs), and 169 long GRBs (lGRBs). Adopting the analysis methods described in Section 3, we detect 169 GRBs with our likelihood analysis above $100 \mathrm{MeV}$. Of these, 155 are lGRBs and 14 are sGRBs. The distribution of the TS obtained by the LTF algorithm is shown in the left panel of Figure 4. The distribution peaks at relatively low values of TS $(\sim 30)$ and then smoothly falls with increasing TS value. Only a handful of GRBs $(\sim 5 \%)$ form a tail at very high TS (above 1000).

Using the LLE technique, 91 GRBs are found below $100 \mathrm{MeV}$. Out of those, 85 are lGRBs and 6 are sGRBs. Moreover, 17 of these GRBs (of which 2 are sGRBs) are found only with the LLE technique and are not detected at higher energies with the LAT standard analysis chain.

Of all 3044 triggers in our initial list, we thus detect $\sim 6 \%$ at high energies with the LAT. About $18 \%$ of the LAT-detected bursts were outside the nominal FOV of the LAT at the time of the trigger. We note that the position in the sky of events that were outside the FOV at trigger time may have entered the FOV at a later time. Moreover, in $10 \mathrm{yr} 220$ triggers initiated an ARR of the satellite, a small fraction $(<10 \%)$ of which are caused by other sources, such as solar flares or particle events. Of these ARRs, 83 successfully resulted in LAT detection. The distribution of the LTF TS values as a function of $\theta$ at the trigger time is shown in the right panel of Figure 4. The highest TS values are seen for GRBs with $\theta<50^{\circ}$.

Furthermore, this catalog includes four GRBs that triggered the LAT directly: one short burst, GRB 090510, and three long ones, GRB 131108A, GRB 160509A, and GRB 160821A. This underscores that onboard LAT GRB detections are relatively rare, implying exceptional brightness in high-energy gammarays. It is worth noting that the very bright GRB 130427A did not result in LAT onboard trigger, since the GBM had triggered and issued an ARR on the first emission peak (Preece et al. 2014), which was very bright at low energies but not particularly strong above $100 \mathrm{MeV}$.

Figure 5 shows the position in equatorial coordinates of 2357 GBM GRB triggers (gray symbols) detected over the $10 \mathrm{yr}$ period of the catalog. A total of $160 \mathrm{lGRBs}$ and 16 sGRBs also detected by LAT are marked by blue and red asterisks, respectively. In fact, out of the 169 likelihood-detected GRBs, 10 did not trigger the GBM instrument. Of these, two GRBs triggered Swift-BAT, namely, GRB 081203A and GRB 130907A, while six GRBs were reported by the IPN: GRB 090427A, GRB 110518A, GRB 120911B, GRB 140825A, the short GRB 160702A, and GRB 180526A. While GRB 120911B did not trigger GBM, it was the only burst to be later found in on-ground analysis of GBM data and announced by Gruber et al. (2012). Furthermore, we report for the first time the detection of GRB 100213C and GRB 111210B. These triggers were reported via private IPN communication, as stated in Section 2.2.

A list of all LAT detections is given in Table 2. For each event, we state the trigger date and time (both in UT and in MET), the final LTF localization with error, the off-axis and zenith angles at trigger time, whether an ARR was issued, the likelihood TS value and LLE significance, the redshift, and the references to the corresponding GCN circulars published by the LAT Collaboration. For those events detected only with the 


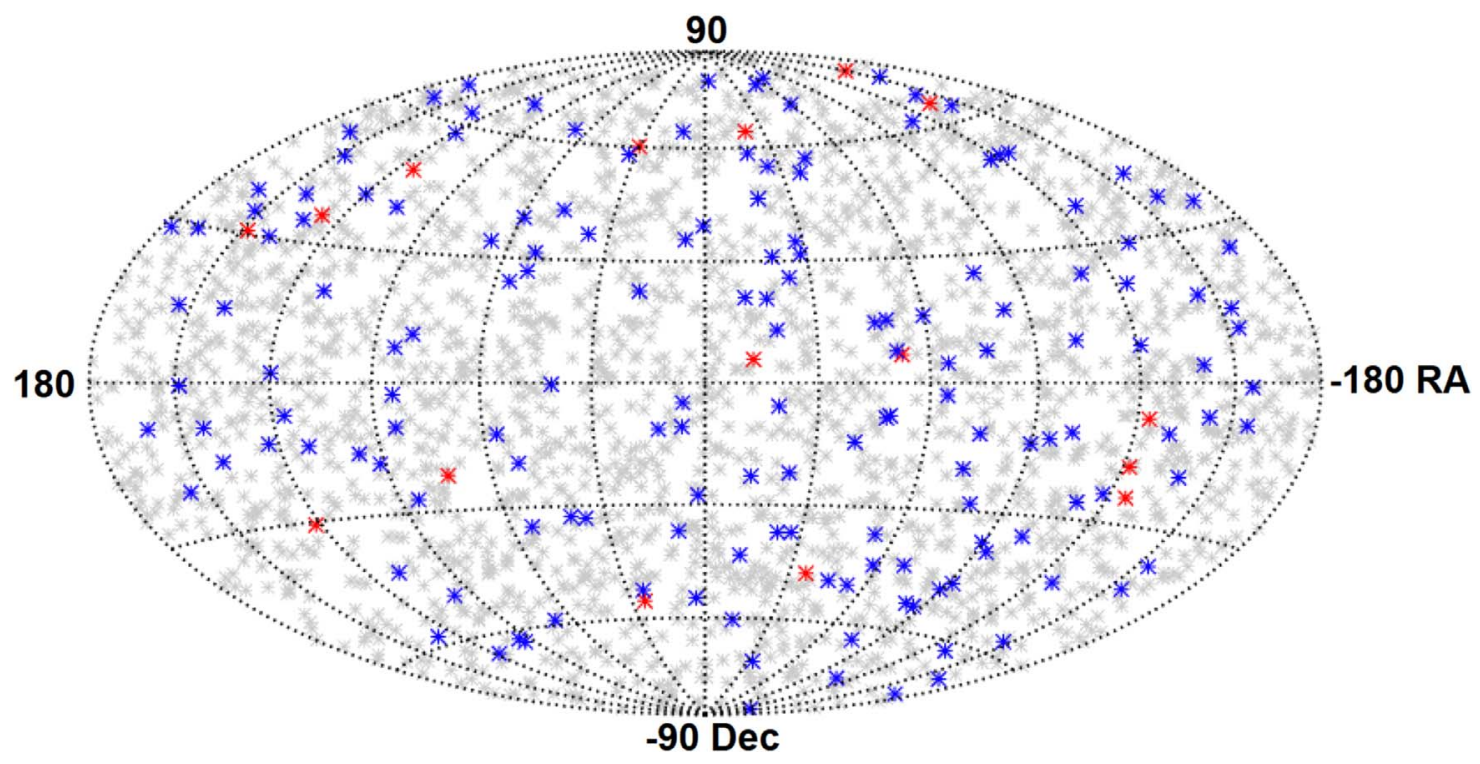

Figure 5. Sky distribution of 2357 GBM-triggered GRBs (from 2008 July 14 to 2018 July 31) in equatorial coordinates (gray asterisks). Blue (red) asterisks indicate 160 (16) long (short) LAT-detected GRBs included in the 2FLGC over the same time period.

LLE technique, we report the best possible localization of the burst as determined by, e.g., GBM or Swift.

In our sample, 34 GRBs have a measured redshift $(19 \%)$, as compared to 10 (29\%) in 1FLGC. For comparison, the fraction of Swift-detected bursts with redshift is $\sim 29 \% .^{74}$ The smaller fraction of LAT bursts with a measured redshift in the 2FLGC with respect to the 1FLGC is not surprising, as $\sim 50$ new GRBs were discovered by our analysis, which have not been previously reported to the community. In addition, the improvements to the analysis techniques enable us to detect fainter GBRs, which are more difficult targets for follow-up observations.

On average, the (90\% containment, statistical only) uncertainty in LAT detections is 0.36 with a range from 0.04 to $2^{\circ} .0$. In order to assess the LAT location accuracy, we also checked for joint detections by Fermi-LAT and Swift and found that 75 bursts $(\sim 40 \%)$ have a BAT position, while 67 bursts $(\sim 36 \%)$ have an XRT position. By comparing LAT and Swift/XRT localizations of the codetected GRBs, we find that $\sim 70 \%$ of the Swift localizations are inside the LAT $90 \%$ confidence region. The majority of the remaining XRT positions are only marginally outside the LAT region, indicating that the LAT localization error is slightly underestimated $\left(\sim 0^{\circ} .1\right)$.

\subsubsection{Comparison with the First LAT GRB Catalog}

The changes and improvements in the 2FLGC mean that the results reported here will differ from those in the 1FLGC. In the time interval of the 1FLGC, 2008 August to 2011 July ( 3 yr), we now recover more events: instead of 28 standard likelihood detections, we now have 50 detections. Three of these new detections are short GRBs, namely, GRB 081102B, GRB 090228A, and GRB 110728A. Four of the new detections come from non-GBM triggers.

The 1FLGC included 21 GRBs also detected with the LLE technique below $100 \mathrm{MeV}$. During the same period, we now find 25 LLE detections. Four of those-GRB 090531B, GRB 100225A, GRB 101123A, and GRB 110529A-are LLEonly bursts as reported also in the 1FLGC, with the first and the last one being short GRBs. The total number of LLE-only

\footnotetext{
${ }^{74}$ https://swift.gsfc.nasa.gov/archive/grb_table/
}

detections is lower with respect to the 1FLGC, where we retrieved seven LLE-only bursts. Indeed, this is not surprising, since we now detect more events with the likelihood analysis thanks to Pass 8 and to the improved LTF pipeline.

As a result of the new analysis, we do not include in the current catalog two events that were included in the 1FLGC: GRB 091208B and GRB 110709A. Both GRBs were long, with estimated LAT durations of $\sim 40 \mathrm{~s}$; however, only three photons were detected for each GRB, and their detection was marked as marginal. The highest-energy photon in GRB 091208B was 1.2 GeV, while GRB 110709A had no detected emission above $500 \mathrm{MeV}$. By selecting Pass 8 data and applying the new detection algorithm, the significance of these two detections further decreased, thus resulting in their exclusion from the 2FLGC.

\subsubsection{LAT Detections after 2011 July}

We have also cross-checked the current catalog with the LAT detections that were publicly announced through GCN Circulars in the time period from 2011 July until 2018 August. Using the standardized catalog analysis described in Section 3.2, we now detect 31 previously unreported GRBs, for which no GCN has been issued. As expected, this is a much smaller relative increase than during the period of the 1FLGC, since Pass 8 data and the improved detection algorithm have been used since 2015 .

Moreover, we do not retrieve eight GRBs that have previously been publicly announced by the Fermi-LAT Collaboration, namely, GRB 120916A (GCN 13777), GRB 130206A (GCN 14190), GRB 131018B (GCN 15357), GRB 140329A (GCN 16047), GRB 150127A (GCN 17356), GRB 150724B (GCN 18065), GRB 161202A (GCN 20229), and GRB 170810A (GCN 21452). In general, these are all GRBs that at the time of detection were reported with low significance, or with few photons. All these cases were analyzed at the time of the GCN writing either on ad hoc time intervals chosen by the burst advocates or on the 10 real-time LTF temporal windows. These differ from the five fixed time intervals chosen for the catalog analysis presented in Section 3.2, thus leading to different results. GRB 130206A and GRB 150127A were previously reported through GCNs as marginal LLE detections, both with a significance $<3 \sigma$, again not matching the current catalog requirements. 

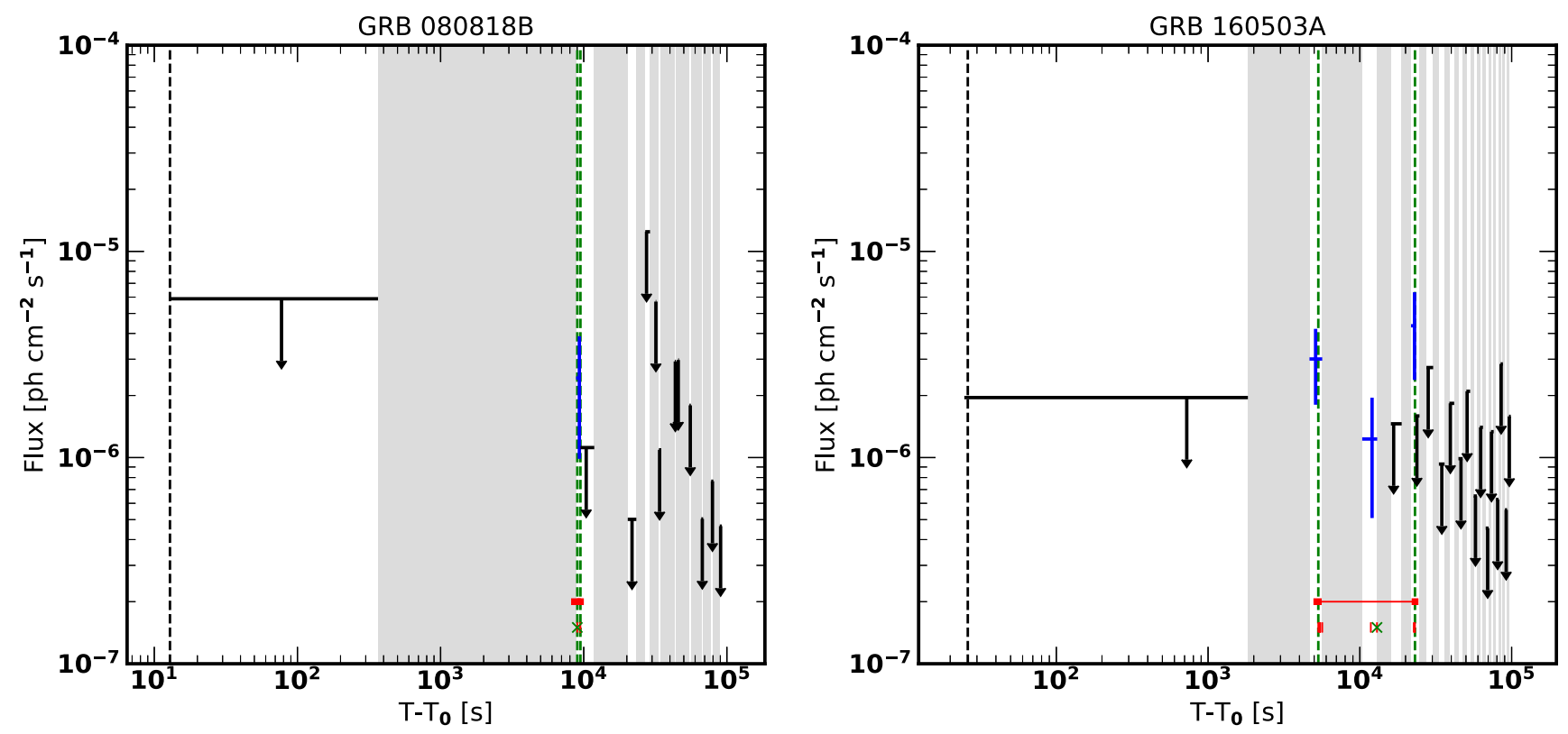

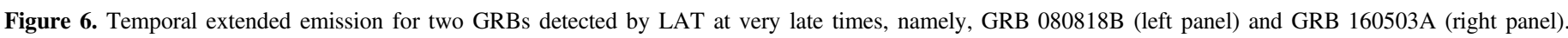
Markers and colors are the same as in Figure 3.
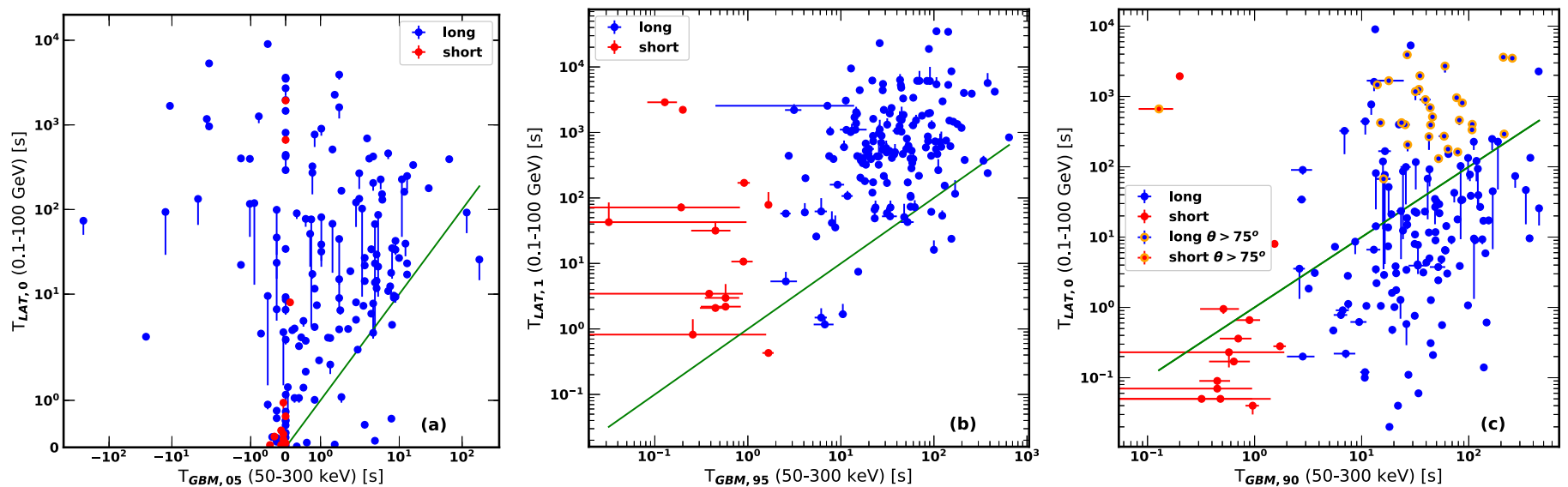

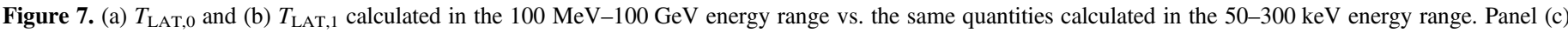

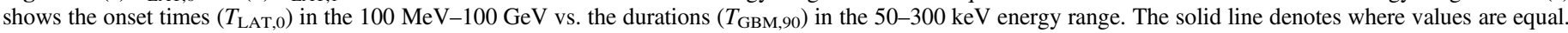

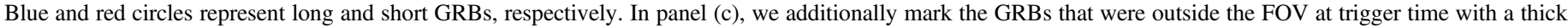
orange contour.

\subsection{LAT Onset Times and Duration}

In the following paragraphs we discuss the temporal properties of the bursts in our sample. As presented in Section 2.5, the classification of GRBs into long and short classes is derived from the low-energy duration as measured by GBM in the 50-300 keV energy band. The LAT durations are calculated in the $100 \mathrm{MeV}-10 \mathrm{GeV}$ energy range. Table 3 summarizes the various temporal characteristics of the GRBs in our catalog. This includes the values of $T_{\mathrm{GBM}, 05}, T_{\mathrm{GBM}, 95}$, and $T_{\mathrm{GBM}, 90}$ for GBM; $T_{\mathrm{LLE}, 05}, T_{\mathrm{LLE}, 95}$, and $T_{\mathrm{LLE}, 90}$ for LLE; and $T_{\mathrm{LAT}, 0}, T_{\mathrm{LAT}, 1}$, and $T_{\mathrm{LAT}, 100}$ for the LAT. Two GRBs, GRB 100213C and GRB 111210B, were reported only by the IPN through private communication: we do not provide any duration information for those. We mark all non-GBM durations in Table 3 with an asterisk in the $T_{\mathrm{GBM}, 05}$ column.

For some GRBs, the LAT detection of the first photon occurs at very late times. This could be due to high-energy photons not being emitted during the initial phase, but it could also be due to observational constraints, where the GRB location is outside the FOV for long intervals. This is illustrated for two GRBs in Figure 6. In both panels, blue points are photon flux measurements, while upper bounds are displayed as black arrows. In the left panel, the first shaded gray area marking when GRB 080818B was outside the FOV spans almost $10 \mathrm{ks}$ $\left(T_{\mathrm{LAT}, 0}=9.0 \pm 0.6 \mathrm{ks}\right)$. The estimated duration of the burst, $T_{\mathrm{LAT}, 100}=500 \pm 200 \mathrm{~s}$, is almost not visible owing to the late time of the detection. Similarly, in the right panel, the first detection of GRB 160503A occurs at $5.3 \mathrm{ks}$, again after a period of several kiloseconds where the burst was first not detected and then outside the FOV. In this case, the duration was $T_{\text {LAT }, 100} \sim 18 \mathrm{ks}$.

In Figure 7(a) we compare the onset times estimated in the LAT energy band (100 MeV-100 GeV) with the ones estimated in the GBM energy band $(50-300 \mathrm{keV})$. A negative $T_{\mathrm{GBM}, 05}$ value in the low-energy band means that the burst 


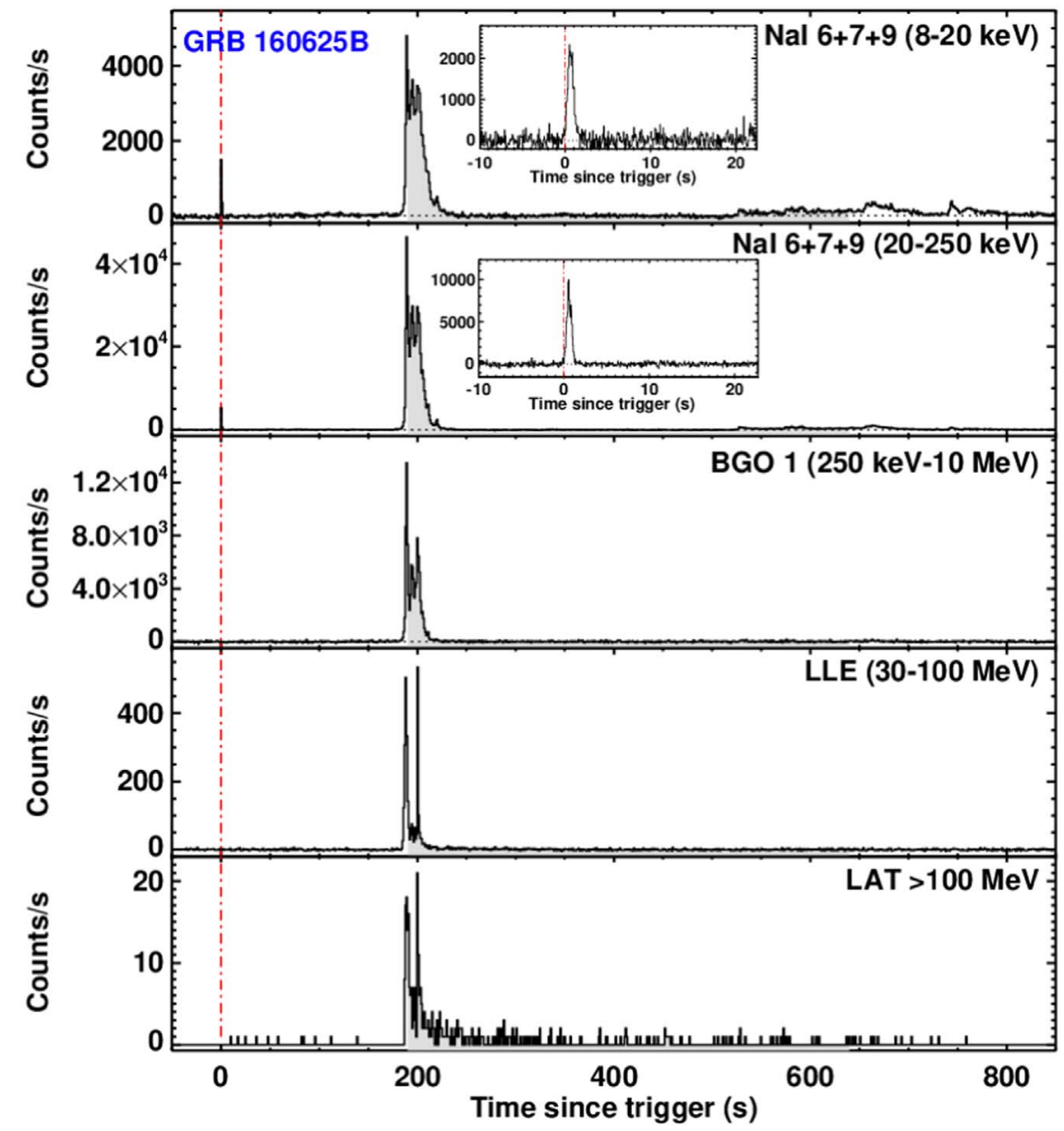

Figure 8. Composite light curve of GRB 160625B: summed GBM/NaI detectors (first two panels), GBM/BGO (third panel), LLE (fourth panel), and LAT rates above $100 \mathrm{MeV}$ (bottom panel). The gray shaded area indicates the $T_{\mathrm{GBM}, 90}$ calculated in the 50-300 keV energy range. The dashed red lines marks the GBM trigger time. A zoom-in around trigger time is shown in two insets for each energy range of the NaI detectors, highlighting the peak detected at low energies that caused the trigger in GBM. This peak does not show any counterpart at higher energies. The association of the low-energy $(<250 \mathrm{keV})$ late-time emission (from 500 to $800 \mathrm{~s}$ post-trigger) with the main GRB emission episode has been cross-checked by the GBM Team (FGGC).

onset occurred before the trigger time. In general, we notice that the high-energy emission starts significantly later with respect to the low-energy one, for both long and short bursts. Burst durations are compared in panel (b) of the same figure. Here, the end of the signal at high energies $\left(T_{\mathrm{LAT}, 1}\right)$ appears to be significantly later than the one measured in the GBM energy band. Both these characteristics were already reported in the 1FLGC. Our results confirm and strongly support the claim that when high-energy emission is observed in GRBs, this emission is delayed and lasts longer compared to that in the lowenergy band.

In Figure $7(\mathrm{c})$ we show the onset time $\left(T_{\mathrm{LAT}, 0}\right)$ of the highenergy emission versus the burst duration $\left(T_{\mathrm{GBM}, 90}\right)$ in the $50-300 \mathrm{keV}$ range. It is worth noting that the $T_{\mathrm{LAT}, 0}$ of the majority of GRBs (both long and short ones) occurs before the prompt emission measured by the GBM is over. Events that were outside the nominal LAT FOV $\left(\sim 75^{\circ}\right)$ at the time of the GBM trigger are marked with thick orange contours. They compose the majority of GRBs where the onset of the highenergy emission came after the low-energy emission had faded, indicating that most such events are due to observational bias. This effect will be further investigated below.

As shown in panels (a) and (b) of Figure 7, there are just a few outliers that have high-energy emission that is not delayed and/or has shorter duration compared to the low-energy band. However, since the procedure to calculate onset times and durations differs between the two energy ranges, we caution that further analysis is needed before strong conclusions can be drawn about individual GRBs. The difference is in most cases less than a few seconds. The most prominent outlier to the right of the line is GRB 160625B, where the GRB $T_{\mathrm{GBM}, 05}$ is $\sim 190 \mathrm{~s}$, whereas $T_{\mathrm{LAT}, 0}$ is $\sim 25 \mathrm{~s}$. However, this burst showed three emission episodes spread over a period of more than $\sim 10$ minutes, as shown in Figure 8 . The first one triggered the GBM, a second one 3 minutes later resulted in LAT onboard trigger, and then the GBM triggered again 10 minutes after the first trigger. It is thus not surprising that the $T_{\mathrm{GBM}, 05}$ is much greater than the arrival time of the first LAT photon.

Short GRBs in general have more similar onset times in LAT and GBM. They also exhibit shorter durations in the highenergy range, although they last still significantly (generally more than an order of magnitude) longer than at lower energies. The short GRB 170127C is the short burst with the longestlasting high-energy duration, more than $2 \mathrm{ks}$.

In our sample, 16 GRBs have high-energy emission lasting over $5 \mathrm{ks}$, and four have durations over $10 \mathrm{ks}$, namely, GRB 160623A ( $\sim 35 \mathrm{ks})$, GRB 130427A ( $\sim 34 \mathrm{ks})$, GRB 140810A ( 18 ks), and GRB 160503A ( $\sim 18 \mathrm{ks})$. Figure 9 shows the temporal extended emission for the two longest bursts, GRB 130427A in the left 

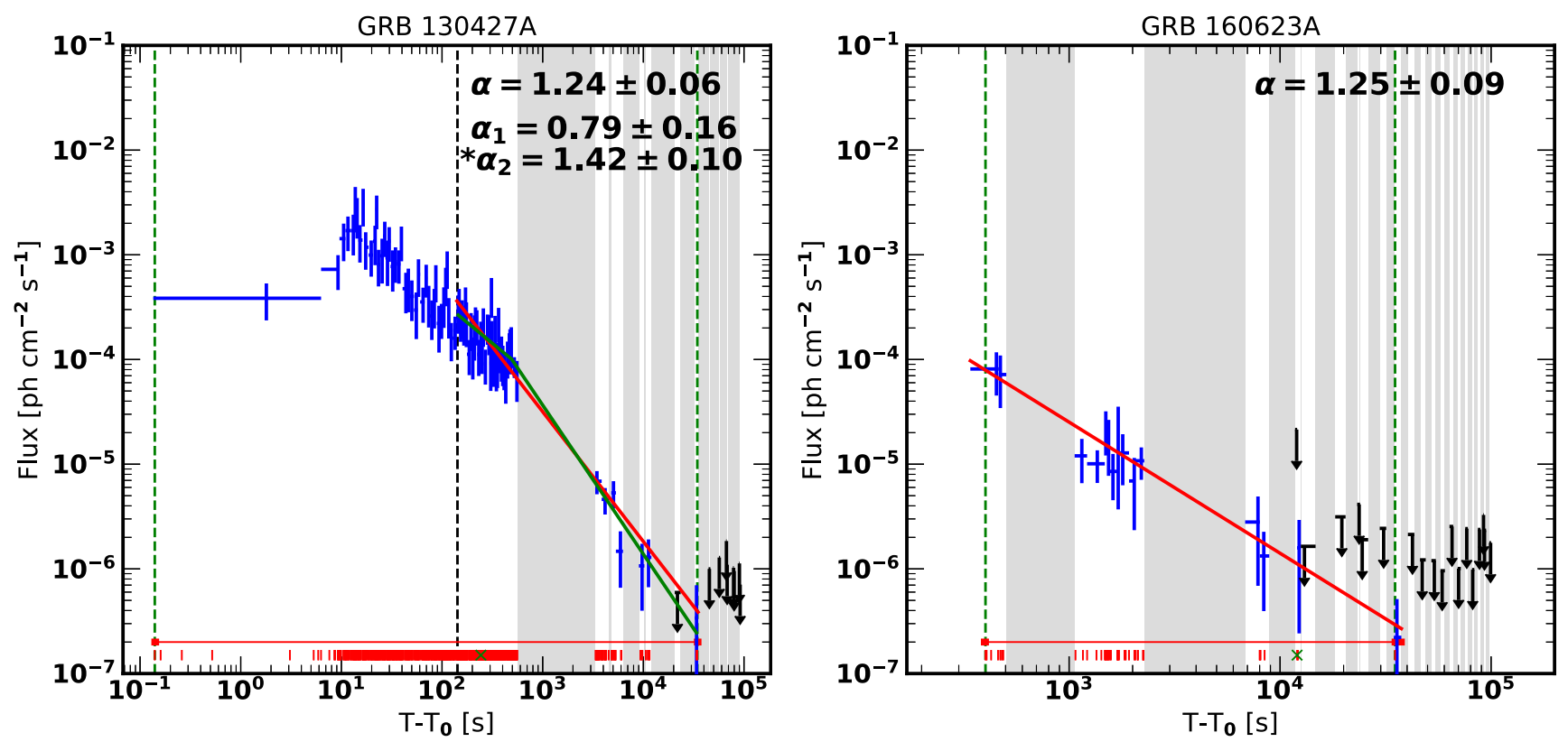

Figure 9. Temporal extended emission for the two longest LAT GRBs, GRB 130427A (left panel) and GRB 160623A (right panel). For the bright GRB 130427A we display the results of the fit for a simple PL model with temporal index $\alpha$ (red line) and with a BPL with indices $\alpha_{1}$ and $\alpha_{2}$ (green line). For GRB 160623A, only the result of the fit with a simple PL is shown.
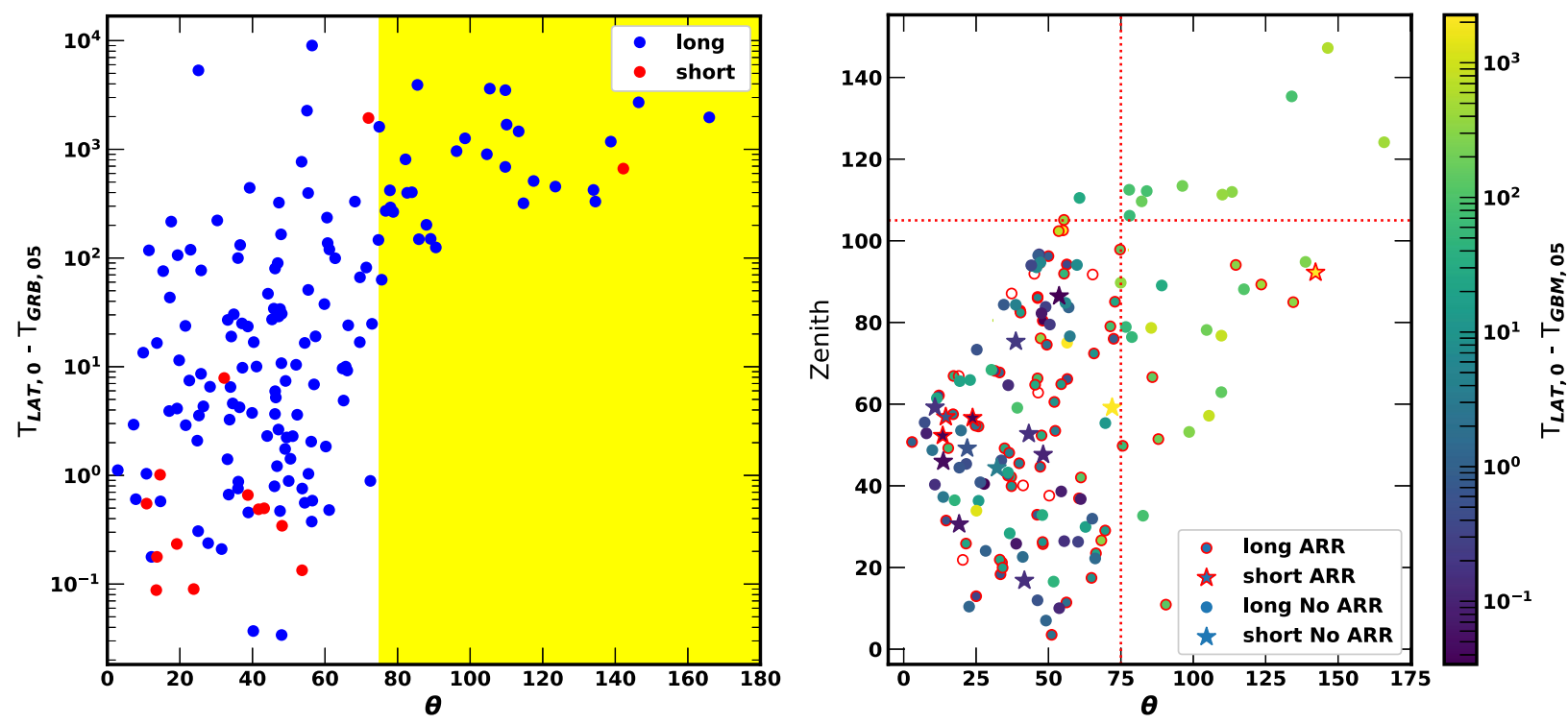

Figure 10. Left panel: difference of the $T_{\mathrm{LAT}, 0}$ and the $T_{\mathrm{GBM}, 05}$ with respect to the incident angle of the GRB at the time of the trigger. The yellow region highlights the GRBs that were outside the LAT FOV $\left(\theta \sim 75^{\circ}\right)$ at the time of the GBM trigger. Right panel: zenith angle $(\zeta)$ vs. incident angle $(\theta)$. The symbols are colored as a function of the difference of the $T_{\mathrm{LAT}, 0}$ and the $T_{\mathrm{GBM}, 05}$ (left panel, $y$-axis). Circles mark long GRBs, while stars mark short GRBs. Bursts that triggered an ARR are marked with red contours. The vertical dashed line marks the LAT FOV, while the horizontal line marks the zenith angle cut used in the analysis $\left(\zeta_{\mathrm{MAX}}=105^{\circ}\right)$. In general, LAT-detected events at $\zeta>105^{\circ}$ and $\theta>75^{\circ}$ are seen at very late times or thanks to ARRs.

panel and GRB 160623A in the right panel. In each panel, we also indicate the fit results to the temporal decay, giving the corresponding model parameters in the top right corner. This will be further discussed in Section 4.7.

As already mentioned, a possible bias in the estimation of the onset time in the LAT is related to the initial position of the GRB at the time of the GBM trigger. For a GRB outside the nominal LAT FOV at trigger time, the first significant detection would happen only when the GRB reenters the FOV. We further illustrate this effect in Figure 10. In the left panel the delay of the LAT onset time with respect to the GBM one is plotted as a function of the incident angle of the GRB, while in the right panel we plot the zenith angle as a function of the incident angle. It is evident that all GRBs that were outside the LAT FOV at trigger time have a large delay ( $\gtrsim 100 \mathrm{~s})$ with respect to the GBM trigger, which corresponds to the time needed for the GRB to reenter the LAT FOV. On the other hand, we also measure significant delays for GRBs that were in the FOV at the time of the GBM trigger, supporting the intrinsic nature of the delay of the high-energy component.

In the right panel of Figure 10, we highlight GRBs that resulted in an ARR, marking each symbol with a red contour. 

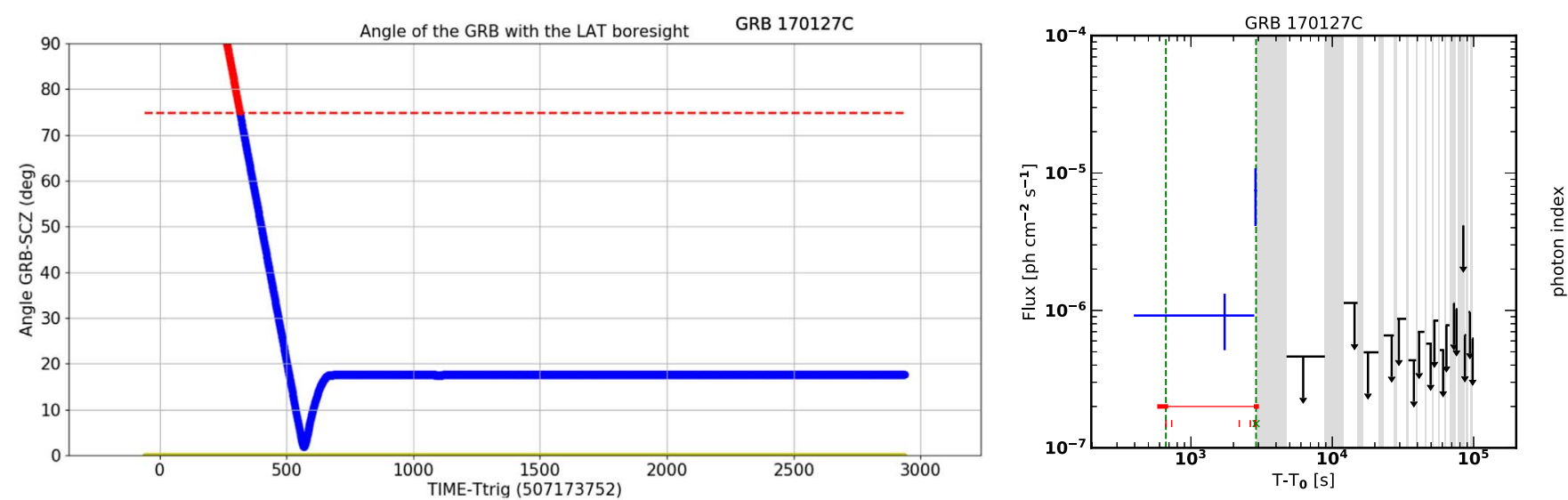

Figure 11. Left panel: off-axis angle for GRB $170127 \mathrm{C}$ as a function of time since the GBM trigger. The horizontal red line corresponds to $\theta=75^{\circ}$. Right panel: temporal extended emission for GRB 170127C. The blue circle is the photon flux measurement with a significance of TS $>10$, while, for lower values of TS, upper bounds are displayed as black arrows. The horizontal red line indicates the estimated duration of the burst, and the thick parts of it correspond to the uncertainty on the $T_{\mathrm{LAT}, 0}$ and $T_{\mathrm{LAT}, 100}$ parameters. The red vertical markers indicate the arrival time of each photon with probability $>90 \%$ to be associated with the GRB, with the green cross being the event with maximum energy.
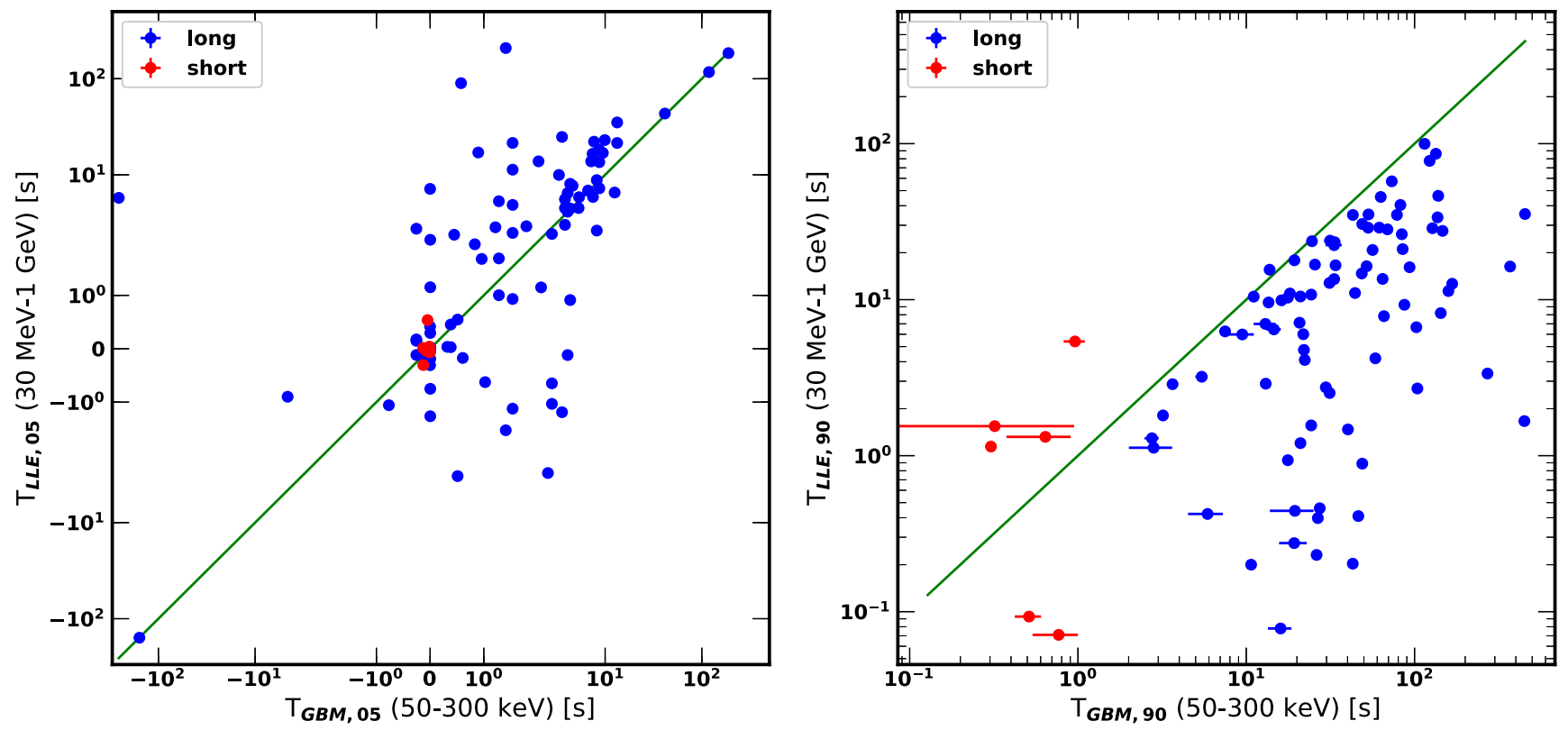

Figure 12. Onset times ( $T_{\mathrm{LLE}, 05}$; left panel) and durations ( $T_{\mathrm{LLE}, 90}$; right panel) calculated using LLE data in the $30 \mathrm{MeV}-1 \mathrm{GeV}$ energy range vs. the corresponding quantities $\left(T_{\mathrm{GBM}, 05}\right.$ and $\left.T_{\mathrm{GBM}, 90}\right)$ calculated using GBM data in the $50-300 \mathrm{keV}$ energy range. The solid green line denotes where values are equal. Blue and red circles represent long and short GRBs, respectively.

Most of the bursts for whom an ARR was issued were in the LAT FOV at the time of the trigger, whereas in seven cases the GRBs were outside the LAT FOV and the detection happened only at later times. To better illustrate this effect, we display the case of the short GRB 170127C in Figure 11. This burst was at a zenith angle of $\zeta \sim 94^{\circ}$ and at an off-axis angle $\theta \sim 142^{\circ}$ when it triggered the GBM (it is the outlier sGRB seen to the far right in both panels of Figure 10). The trigger resulted in an ARR, and the spacecraft slewed to move the location of the burst close to the center of the FOV (at $\sim 17^{\circ}$ ). This can be seen in the left panel of Figure 11, where the blue circles show the evolution of $\theta$ as a function of time after the trigger. In the right panel, we show the photon flux light curve resulting from the time-dependent analysis. The first detection is at $\sim 400 \mathrm{~s}$, well beyond the end of the GBM signal $\left(T_{\mathrm{GBM}, 95}=0.13 \mathrm{~s}\right)$.

\subsection{LLE Onset and Duration}

If we restrict our considerations to the LLE analysis, where the bulk of the emission is in the energy range from $30 \mathrm{MeV}$ to $1 \mathrm{GeV}$, we see that the left panel of Figure 12 shows how the onset times are relatively similar to the onset times as measured by the GBM. Here, two GRBs are not shown: GRB 120624B and GRB 150513A. Both GRBs triggered Swift before they triggered GBM, 257 s (Barthelmy et al. 2012) and $157 \mathrm{~s}$ (Kocevski et al. 2015) before the GBM trigger time, respectively. As a result, since all our calculations are referred to GBM trigger times, $T_{\mathrm{LLE}, 05}$ is negative and omitted from the figure (see Table 3).

In contrast to the emission above $100 \mathrm{MeV}$, the right panel of Figure 12 indicates that the duration of the signal in LLE is systematically shorter than the duration of the signal in the GBM, as was seen also in the 1FLGC. If we assume that the 

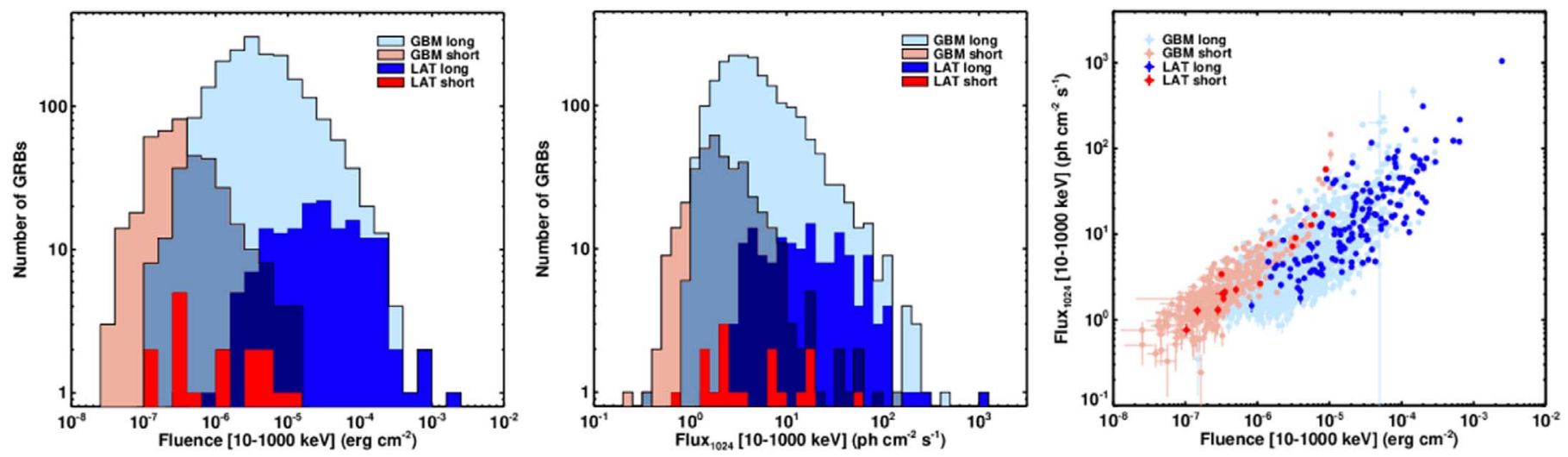

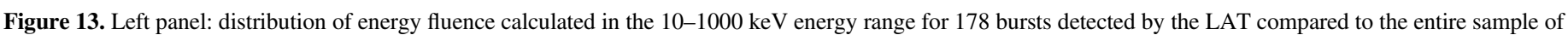

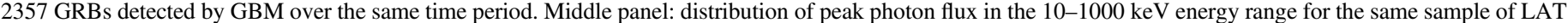

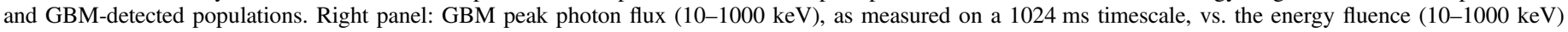
derived using a spectral model fit to a single spectrum over the entire duration of the burst.

LLE emission is dominated by the same emission episodes as in the GBM, we can infer that the pulses that make up the time profile of the prompt emission are systematically shorter in the LLE range than at lower energies. This behavior has previously been reported by Norris et al. (1996) and Norris (2002) using BATSE data, as well as for several LAT-observed GRBs (e.g., Axelsson et al. 2012; Bissaldi et al. 2017; Vianello et al. 2018).

\subsection{Comparison to the GBM Population}

Since the majority of our triggers come from the GBM, and the GBM has observed nearly all GRBs in our sample, we examine how the LAT-detected bursts are drawn from the general GBM population covering the same $10 \mathrm{yr}$ time period. For this comparison, we extracted the peak photon flux, as measured on a $1024 \mathrm{~ms}$ timescale, and energy fluence measured by the GBM in the $10-1000 \mathrm{keV}$ energy range from the FGGC. Here the GBM fluence is derived from the parameters of the best-fit spectral model applied to GBM data over a time interval where the signal-to-noise ratio $(\mathrm{S} / \mathrm{N})$ exceeds a predefined value $(\mathrm{S} / \mathrm{N}>3.5$; see Gruber et al. 2014, for more details). This requirement ensures that there are enough counts to perform a spectral fit, but as a result, the time interval does not always coincide with $T_{\mathrm{GBM}, 90}$. Note that eight GRBs, two triggered by Swift and six by the IPN, were not detected by the GBM and are omitted from this comparison and from the following figures.

Figure 13 shows the distribution of the energy fluence (left panel) and of the peak photon flux (middle panel) for 178 bursts detected by the LAT compared to the entire sample of 2357 GRBs detected by GBM over the same time period. Here we have also made a distinction between short and long bursts for both the LAT (16 sGRBs and 162 lGRBs) and GBM (400 sGRBs and 1957 lGRBs) populations, showing a bifurcation in the range of flux and fluence values covered by these two classes of bursts. The right panel shows the peak photon flux plotted against the energy fluence for the LAT bursts compared again to the entire GBM burst catalog. Again, we separate short and long bursts for both the LAT and GBM populations.

These comparisons show that although the majority of the LAT-detected GRBs come from the GBM-detected bursts with the highest peak flux and fluence, they cover a large range. LAT-detected short (long) bursts are present with a fluence
$>10^{-7} \mathrm{erg} \mathrm{cm}^{-2}\left(>8 \times 10^{-7} \mathrm{erg} \mathrm{cm}^{-2}\right)$ and with a peak flux $>0.8$ photons $\mathrm{cm}^{-2} \mathrm{~s}^{-1}\left(>1.5\right.$ photons $\left.\mathrm{cm}^{-2} \mathrm{~s}^{-1}\right)$. The LATdetected long GRBs cover more than two orders of magnitude in both distributions, and the prominence of bright GRBs is even less pronounced in the short GRB sample. The spread is also evident from the right panel of Figure 13, where the cluster of LAT events is only slightly shifted with respect to the GBM one. The burst with the highest fluence (and flux) is GRB 130427A. It is worth noting that Figure 13 does not include any selection on the $\theta$ angle.

\subsection{Flux, Fluences, and Photon Indices from the Time- integrated Analysis}

The results of the likelihood analysis are summarized in Table 4. For each time window, we report the number of detected and predicted LAT events in the ROI, the resulting TS, the spectral index obtained using a PL fit, and the LAT flux and fluence calculated in the $100 \mathrm{MeV}-100 \mathrm{GeV}$ energy range. For $34 \mathrm{GRBs}$ with known redshift we also report the total radiated energy $\left(E_{\text {iso }}\right)$.

Figure 14 shows the distributions of fluxes (left panels) and fluences (right panels) as a function of the measured duration of the signal in the "GBM" (top row) and "LAT" (bottom row) time windows. LAT fluxes decrease with increasing burst duration in both time windows, as expected. In the "GBM" time window, the LAT fluence seems to be clustered around a value of $10^{-6} \mathrm{erg} \mathrm{cm}^{-2}$ for the majority of lGRBs (regardless of duration), while sGRBs show slightly lower values. Both groups have bursts that are very much brighter than the average. At late times, there is instead a tendency for the fluence to increase with duration. The same conclusion can be drawn from the fluence values in the "LAT" time window, where most of the values are distributed around $\sim 5 \times$ $10^{-6} \mathrm{erg} \mathrm{cm}^{-2}$ and there is a less evident spread toward higher values.

Comparing our results to Figure 11 in the 1FLGC, we find that the four "hyperfluent" GRBs are no longer outliers. Instead, they are part of a continuous distribution. The range in both flux and fluence has also increased dramatically as compared to the sample in the 1FLGC.

In Figure 15 we then compare the LAT fluence calculated in the $100 \mathrm{MeV}-100 \mathrm{GeV}$ energy range during the "GBM" time window with the GBM fluence calculated between $10 \mathrm{keV}$ and 

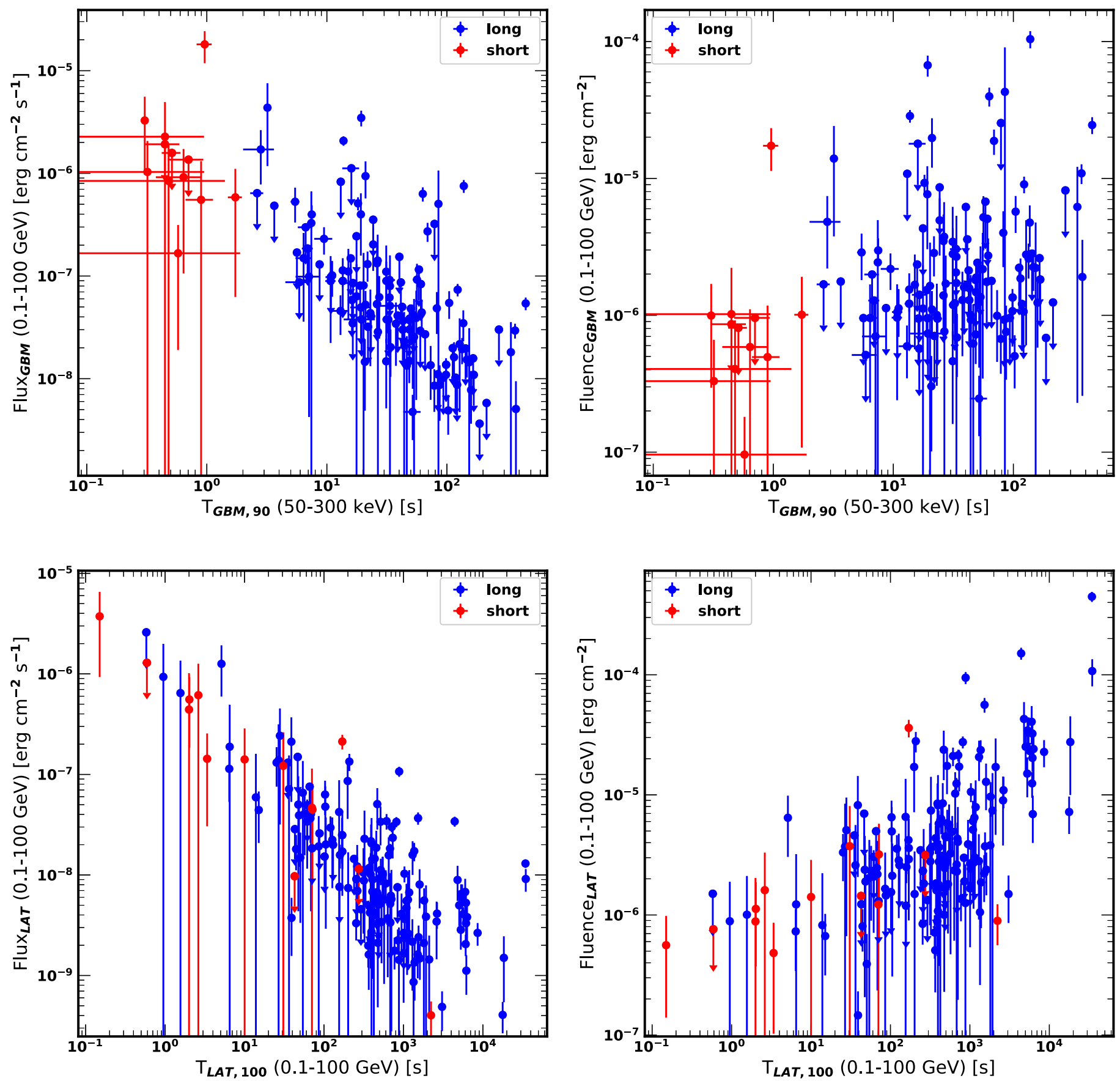

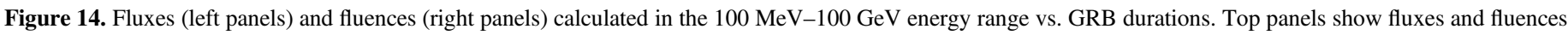

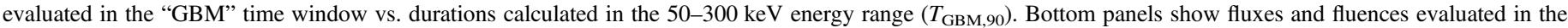

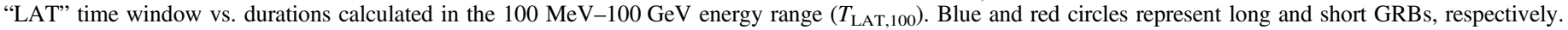

$1 \mathrm{MeV}$ (left panel) and with the LAT fluence calculated in the same energy range during the "EXT" time window (right panel). In the left panel, it can be noted that the "GBM" time window is dominated by the low-energy emission, with the $100 \mathrm{MeV}-100 \mathrm{GeV}$ energy range contributing only a small fraction of the emission for the majority of long GRBs. Indeed, most events are clustered to the left of the solid and dashed lines, which indicate equality and a factor of 10 less, respectively. For short GRBs this difference seems less pronounced, and several lie close to the solid line of equality. Comparing the "GBM" and "EXT" time windows in the right panel, the points are instead much closer to the line of equality, suggesting that the high-energy emission in the two time windows is comparable. As in Figure 14, the four "hyperfluent" GRBs of the 1FLGC (GRB 080916C, GRB 090510, GRB 090902B, and GRB 090926A) are no longer outliers.

In Figure 16 we also compare the photon index measured by the LAT during the "GBM" time window $\left(\Gamma_{\mathrm{GBM}}\right)$ and the "EXT" time window $\left(\Gamma_{\text {EXT }}\right)$. Both indices are plotted as a function of the GRB duration as calculated in the $50-300 \mathrm{keV}$ energy range (top panels) and of the LAT flux calculated in the "GBM" (bottom left panel) and "EXT" (bottom right panel) 

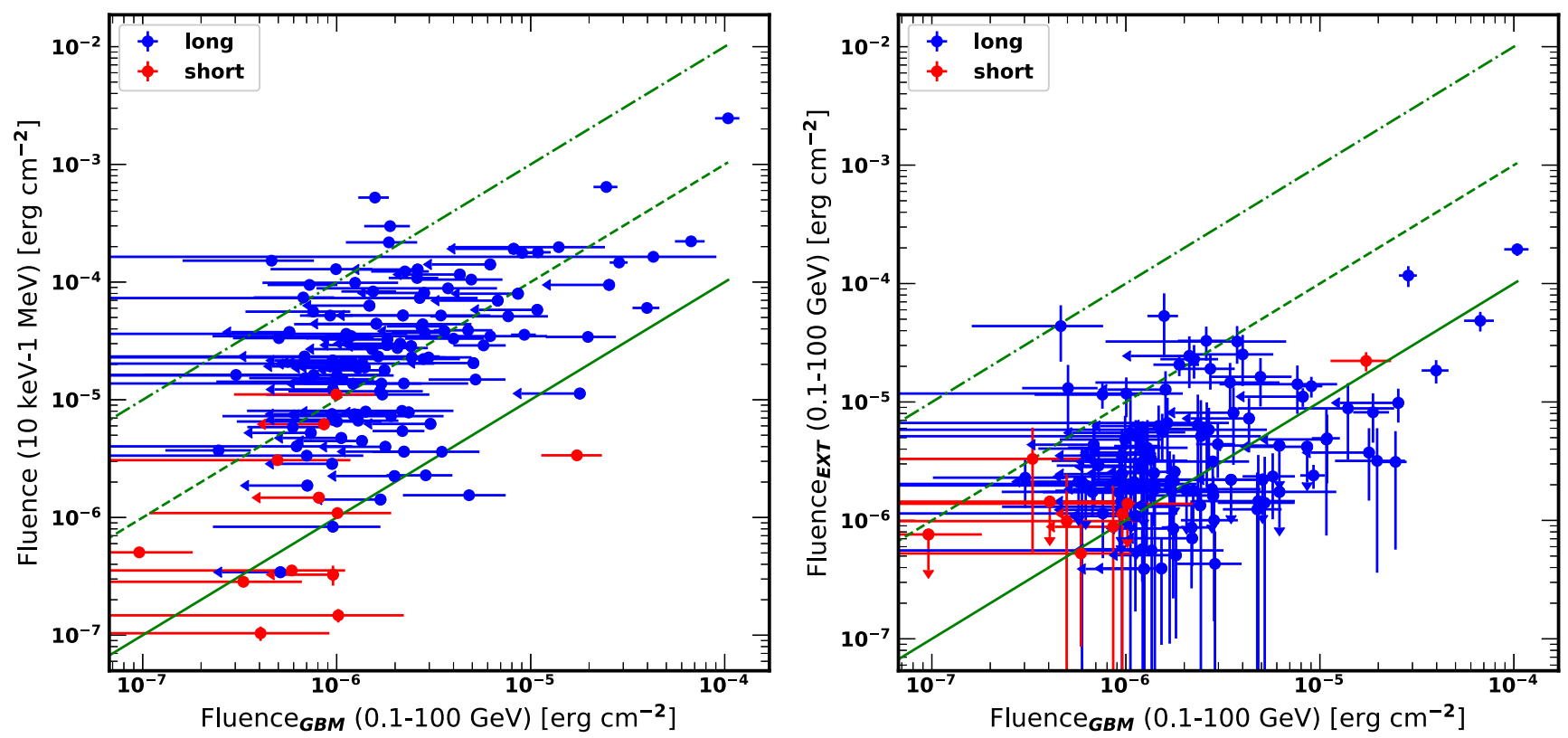

Figure 15. Left panel: fluences calculated in the $10-1000 \mathrm{keV}$ energy range vs. fluences calculated in the $100 \mathrm{MeV}-100 \mathrm{GeV}$ energy range. All values are estimated in the "GBM" time window. Right panel: fluences calculated in the $100 \mathrm{MeV}-100 \mathrm{GeV}$ energy range evaluated in the "EXT" time window vs. the same quantities evaluated in the "GBM" time window. The solid green lines denote where values are equal. The dashed and dot-dashed green lines are shifted by factors of 10 and 100 , respectively.

time windows. The photon index shows no sign of being correlated with either the GBM duration or the flux in either time window, and it is similar for long and short GRBs. The value is indeed similar between the two time windows but is slightly harder in the "EXT" window. In the "GBM" time window, the values of the photon index are more scattered, with a mean value of $\Gamma_{\mathrm{GBM}}=-2.49$ and a 10th (90th) percentile of $-3.22(-1.86)$. In the "EXT" time window, the values are more uniform, with a mean of $\Gamma_{\text {EXT }}=-2.03$ and a 10 th (90th) percentile of $-2.45(-1.6)$. For comparison we recall the same values reported in the 1FLGC: $-2.08 \pm 0.04$ in the "GBM" time window and $-2.00 \pm 0.04$ in the "EXT" time window. While the latter is in agreement with the current value, the photon index during the "GBM" time window was much harder than the one we derive in the 2FLGC. Interestingly, it showed a weak inverse correlation with the duration of the burst (see Figure 26 of the 1FLGC). This correlation is now less evident in the larger sample of bursts, but the $\Gamma_{\mathrm{GBM}}$ distribution still underlines the agreement with previous findings that the spectra of short-duration GRBs tend to be harder. No clear trend can be seen in the comparison with flux (bottom panels), except a slight tendency for low-flux GRBs to show harder spectra when looking in the "EXT" window.

\subsection{Energetics}

In order to more closely study the energetics of the bursts in this catalog, and to put the detections in a wider context, we focus on the GRBs with known redshift. We decided to compare our sample to other bursts with measured redshift detected by Swift and GBM. As of the end of 2018 July, SwiftBAT has detected 1246 GRBs, of which $\sim 35 \%$ have a measured redshift. In the case of the GBM-detected GRBs, only $\sim 5 \%$ have measured redshift. The redshift distributions of 405 bursts detected by Swift-BAT (gray histogram), 116 bursts detected by GBM (cyan histogram), and 34 bursts detected by
LAT (blue histogram) are shown together in Figure 17. We see no obvious difference between the three distributions.

We next compare the isotropic radiated energy $\left(E_{\text {iso }}\right)$ and the bolometric gamma-ray peak luminosity $\left(L_{\text {iso }}\right)$ of LAT-detected GRBs to the same quantities in the Swift and GBM samples. The values for $E_{\text {iso }}$ are computed according to Equation (3) in the $1 \mathrm{keV}-10 \mathrm{MeV}$ energy range. In the case of GBM-detected GRBs, we adopt the fluence listed in the FGGC as computed from the best-fit spectral model, which is usually calculated on a slightly different time interval with respect to the burst $T_{\mathrm{GBM}, 90}$, according to the burst brightness.

In order to compute $E_{\text {iso }}$ of Swift-detected events (with no GBM observation), we used the parameters of the best-fit spectral models obtained in the $15-350 \mathrm{keV}$ energy range reported in the Swift-BAT online catalog ${ }^{75}$ (see Lien et al. 2016, for more details). These are calculated over a time interval corresponding to a duration that contains $100 \%$ of the burst emission. For both the GBM and BAT $E_{\text {iso }}$ calculation, we only consider bursts for which the spectral parameters are globally well constrained (see Gruber et al. 2014). Thus, we find 116 (405) GBM (BAT) GRBs that satisfy these criteria, out of which 108 (376) are lGRBs and 8 (29) are sGRBs. The LAT sample comprises 32 lGRBs (2 of the 34 were not detected by the GBM, as previously discussed) and only one sGRB (090510).

We also calculate the isotropic luminosity $L_{\text {iso }}$, which takes into account the GRB prompt emission spectrum and is defined in a $1 \mathrm{~s}$ time interval centered around the time of the peak flux. It can be expressed as

$$
L_{\text {iso }}=4 \pi d_{L}^{2} P\left(E_{1}, E_{2}, z\right)
$$

\footnotetext{
$\overline{75}$ https://swift.gsfc.nasa.gov/results/batgrbcat/
} 

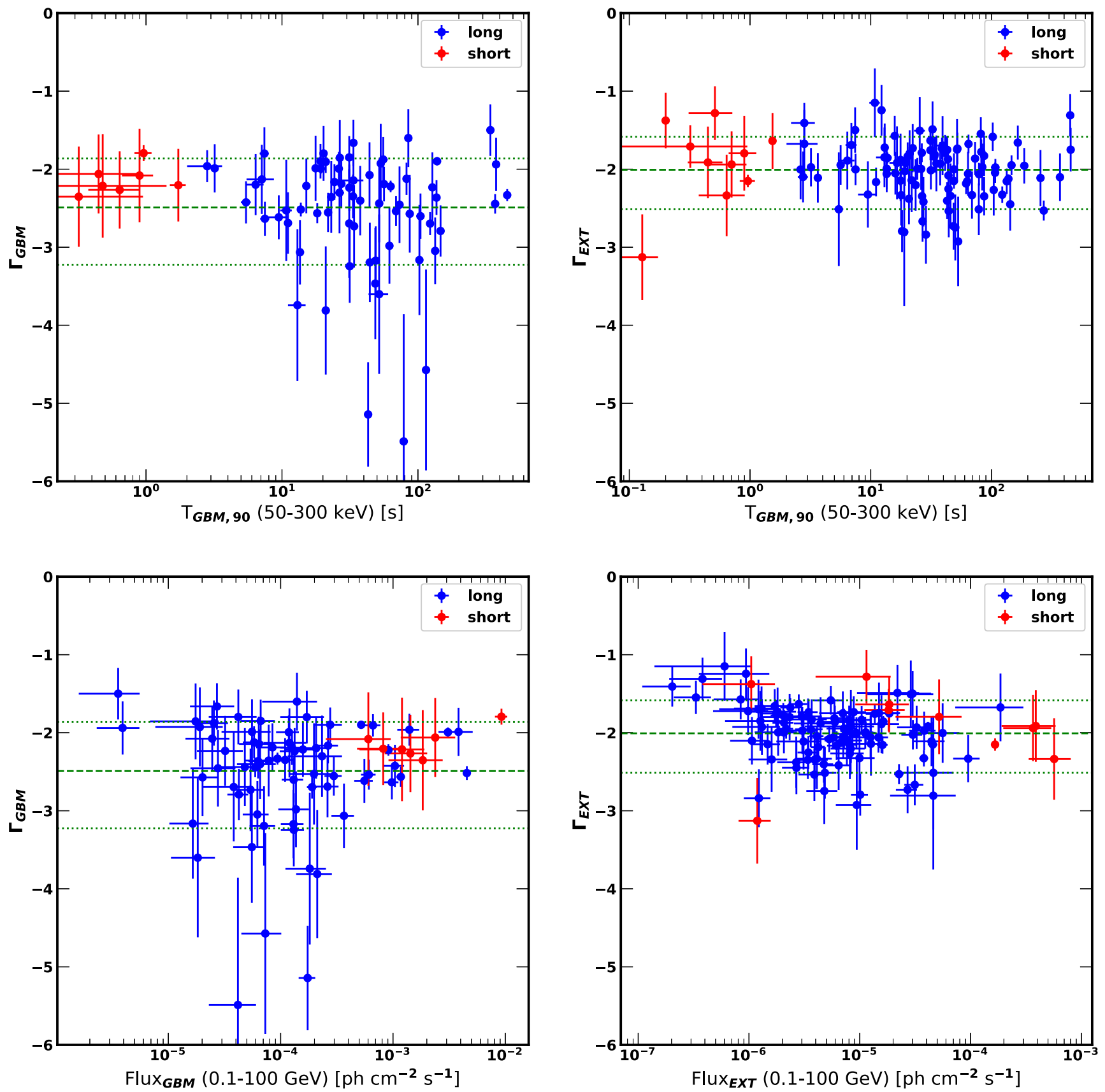

Figure 16. Photon indices $\Gamma_{\mathrm{GBM}}$ (left panels) and $\Gamma_{\mathrm{EXT}}$ (right panels) calculated in the $100 \mathrm{MeV}-100 \mathrm{GeV}$ energy range vs. duration calculated in the $50-300 \mathrm{keV}$ energy range (top panels) and LAT $100 \mathrm{MeV}-100 \mathrm{GeV}$ fluxes (bottom panels). These are calculated in the "GBM" (bottom left) and "EXT" (bottom right) time windows, respectively. The green dashed lines denote the mean values and the dotted lines the 10th and 90th percentiles of each distribution. Blue and red circles represent long and short GRBs, respectively.

where $P\left(E_{1}, E_{2}, z\right)$ represents the bolometric peak flux, defined as

$$
P\left(E_{1}, E_{2}, z\right)=\int_{E_{1} /(1+z)}^{E_{2} /(1+z)} E N(E) d E .
$$

As with $E_{\text {iso }}, L_{\text {iso }}$ is computed in the $1 \mathrm{keV}-10 \mathrm{MeV}$ energy range, for GBM-detected GRBs, using the $1 \mathrm{~s}$ peak flux of the best-fit model as reported in the FGGC. We again consider only GRBs whose time-integrated spectra are well defined, as reported in the GBM and Swift-BAT GRB catalogs. This leaves us with 394 BAT GRBs and with the same number (116) of
GBM GRBs. The slightly lower number of BAT GRBs is expected, as the time interval (and thereby the number of photon counts) is smaller.

Figure 18 shows the distribution of $E_{\text {iso }}$ (left panel) and $L_{\text {iso }}$ (right panel) as a function of redshift. Swift-BAT and GBM bursts are indicated by gray and black points, respectively, with long (short) bursts marked with filled (open) symbols. LAT long and short bursts are marked with the standard blue and red circles, respectively, used in this paper. LAT-detected GRBs populate the top portion of both distributions, as was previously seen in the 1FLGC. At that time, this figure only contained nine LAT-detected GRBs with redshift. It is 


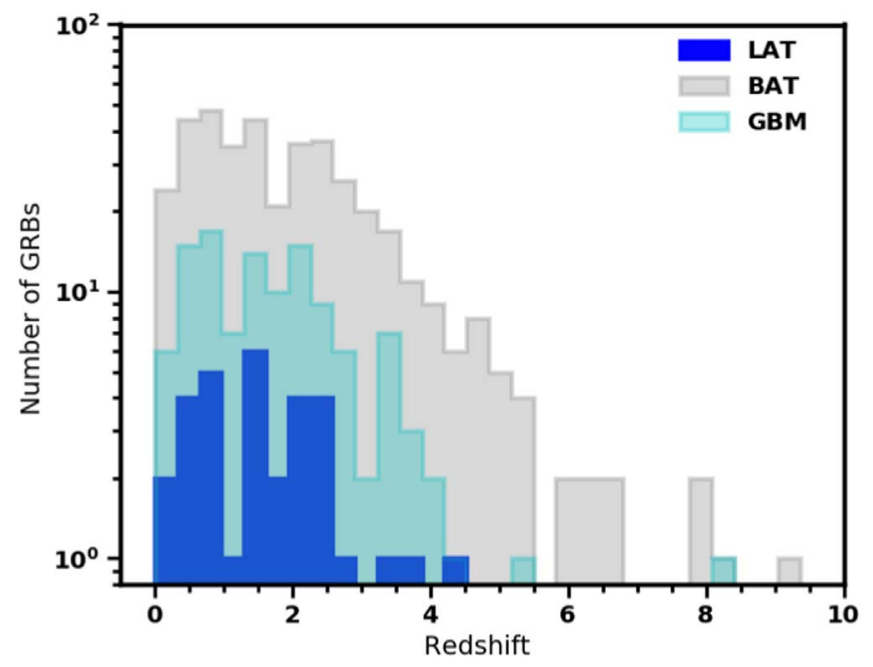

Figure 17. Redshift distribution of $34 \mathrm{GRBs}$ detected by LAT (blue histogram), 405 GRBs detected by Swift-BAT (gray histogram), and 116 GRBs detected by GBM (cyan histogram).

worth noting that quite a few bursts have a moderate $1 \mathrm{keV}-$ $10 \mathrm{MeV} E_{\text {iso }}\left(\lesssim 10^{-53} \mathrm{erg}\right)$ yet have nevertheless been detected by the LAT.

\subsection{Time-resolved Light Curves}

We now turn to the temporal decay of the high-energy extended emission. Using the analysis described in Section 3.6.2, we were able to determine the evolution of the flux as a function of time for 115 long and 11 short GRBs in our sample. This is shown in the two panels of Figure 19, displaying the temporal decay of long (left panel) and short (right panel) bursts separately. Each event is marked with a different color. The light curves of both sGRBs and lGRBs show a fairly large spread in the observer frame.

In order to determine the corresponding temporal decay index, we perform a fit of all the light curves maximizing the $\chi^{2}$, with two different spectral models, namely, (1) a simple PL,

$$
F(t)=F_{0}\left(\frac{t}{T_{0}}\right)^{-\alpha},
$$

where $\alpha$ is the temporal decay index, $T_{0}$ is the GRB trigger time, and $F_{0}$ is the normalization flux; and (2) a BPL,

$$
F(t) \propto t^{-p}\left\{\begin{array}{l}
p=\alpha_{1} \text { for } t<T_{b} \\
p=\alpha_{2} \text { for } t \geqslant T_{b},
\end{array}\right.
$$

with index $\alpha_{1}$ for times before the break time $T_{b}$ and index $\alpha_{2}$ afterward. If there are at least three flux points (with TS $>10$ ) in the light curve after the $T_{\mathrm{GBM}, 95}$, we fit a PL, and if there are at least four flux points, we also try a BPL.

The results of the fits are presented in Table 5. By fitting the flux temporal decay with a BPL, we find a significant improvement in 12 cases. We show three examples in Figure 20 (GRB 090510, GRB 150627A, and GRB 180720B; a fourth, GRB 130427A, has already been shown in the left panel of Figure 9). The BPL fit is indicated with a solid green line, and the corresponding fit values are given in the upper right corner of each panel. The PL fit is shown with a red solid line for comparison. In all but two cases the light curves manifest a steep-to-shallow decay, while for
GRB 171120A and GRB 180720B (right panel in Figure 20) the decay steepens after the break.

The distribution of late-time temporal decay indices ( $\alpha$ or $\alpha_{2}$ ) is displayed in Figure 21, together with a Gaussian fit (black dashed line) to the distribution. The distribution comprises 88 GRBs, including 86 long and 2 short bursts. Among the long (short) GRBs, 77 (1) are best fit with the PL model, while 11 (1) prefer a BPL model. This is a large increase compared to the 1FLGC, where only nine GRBs had enough data to allow the decay index to be determined, ranging from 0.8 (for GRB 090902B) to 1.8 (for GRB 080916C) and with a mean value of $\sim 1.1$. We now find a mean value of $0.99 \pm 0.04$ with a standard deviation of $0.80 \pm 0.07$, still in agreement with the results presented in the 1FLGC.

These results and their interpretation will be discussed in more detail in Sections 5.6 and 6.3, but it is worth noting that there are several cases in which a BPL would likely be required if the data in the "GBM" time window had been included. Furthermore, several GRBs show features in the light curve that deviate from both a PL and BPL. Four light curves exemplifying both these cases are displayed in Figure 22.

In the figure, GRB 131108A (top left) exemplifies how some bursts display strong variability in the LAT $>100 \mathrm{MeV}$ light curve during the GBM emission. GRB 140206B (top right) instead shows the possible presence of a late-time high-energy pulse. GRB 160625B (bottom left) shows a strong pulse with a very sharp decay. GRB 160816A (bottom right panel) would clearly require a break to accommodate the data in the "GBM" time window. However, in the "EXT" time window used for the fits, a PL model is statistically preferred. These peculiar features are all observed for the first time in the 2FLGC. Some light curves, like GRB 140206B (top right panel in Figure 22), show a sharp step between the end of the "GBM" and the beginning of the "EXT" time window (marked by the vertical black dashed line). In these cases, a fit of the complete data set would again favor a BPL model rather than the PL one currently used in the "EXT" time window. In a few even more extreme cases, the light curve could not be fit at all in the "EXT" time window, since there is only a single point in the light curve after the "GBM" time window.

In Figure 23, we show the $100 \mathrm{MeV}-100 \mathrm{GeV}$ luminosity evolution for the $34 \mathrm{GRBs}$ in our sample with measured redshift. Among those, there is only one short burst, namely, GRB 090510. The three panels of the figure show first the light curves in the observer frame (left), the evolution as a function of time in the source frame (middle), and finally the luminosity divided by isotropic energy ( $E_{\text {iso }}$; right), calculated in the $1 \mathrm{keV}-10 \mathrm{MeV}$ energy range. Each correction brings the light curves closer together, and in the right panel there is a remarkable alignment of all the GRBs. This analysis was done following the one presented by Nava et al. (2014), where a similar result was found. It is worth noting that in the right panel of Figure 23 one of the GRBs, GRB 160623A, does not align with the others, indicating a possible outlier. This burst was occulted by Earth for a large part of its duration, and the GBM trigger occurred $\sim 50 \mathrm{~s}$ after the start of the GRB based on the Konus-Wind light curve. This likely leads us to underestimate the total energy release $E_{\text {iso }}$ of the burst and thus to overestimate the normalization of the light curve. In the right panel we also include a linear fit to all $34 \mathrm{GRBs}$, indicated by the solid line. The decay index is $1.25 \pm 0.03$. For 

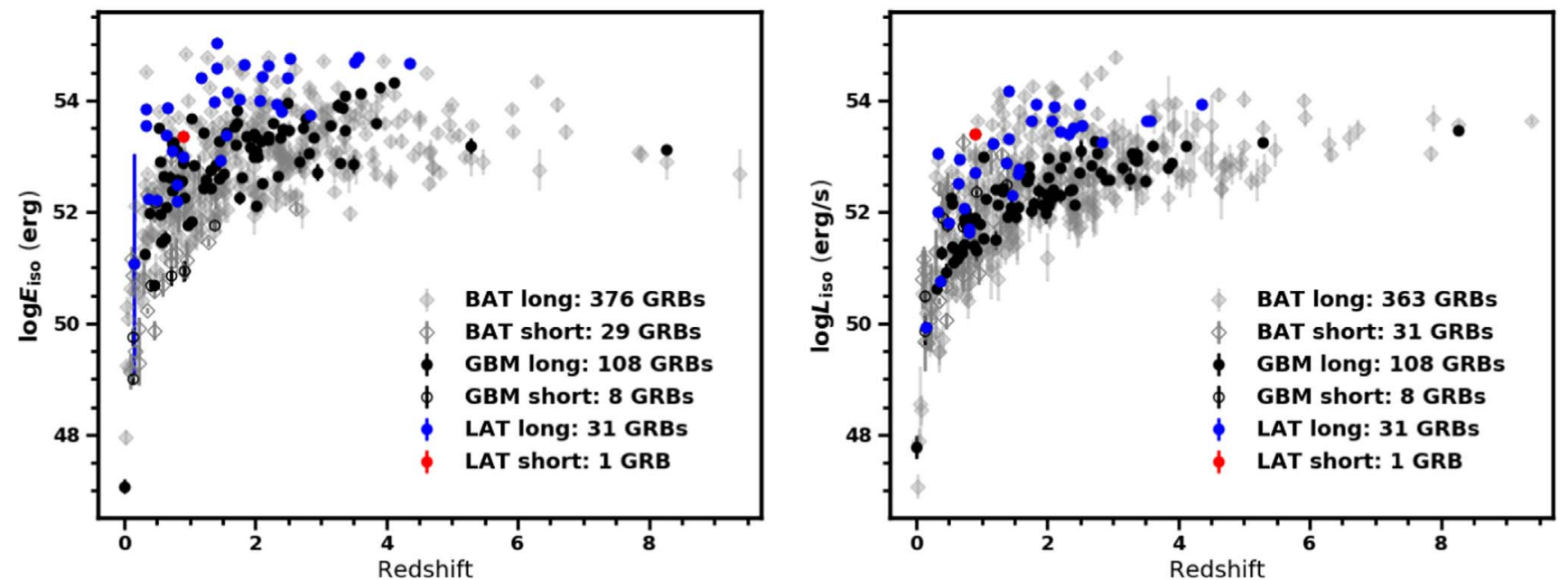

Figure 18. Left panel: isotropic radiated energy vs. redshift. Right panel: isotropic luminosity vs. redshift. Blue/red circles indicate the LAT long/short GRBs, and black and gray circles indicate GBM/Swift-BAT GRBs, with short bursts always marked by open symbols.
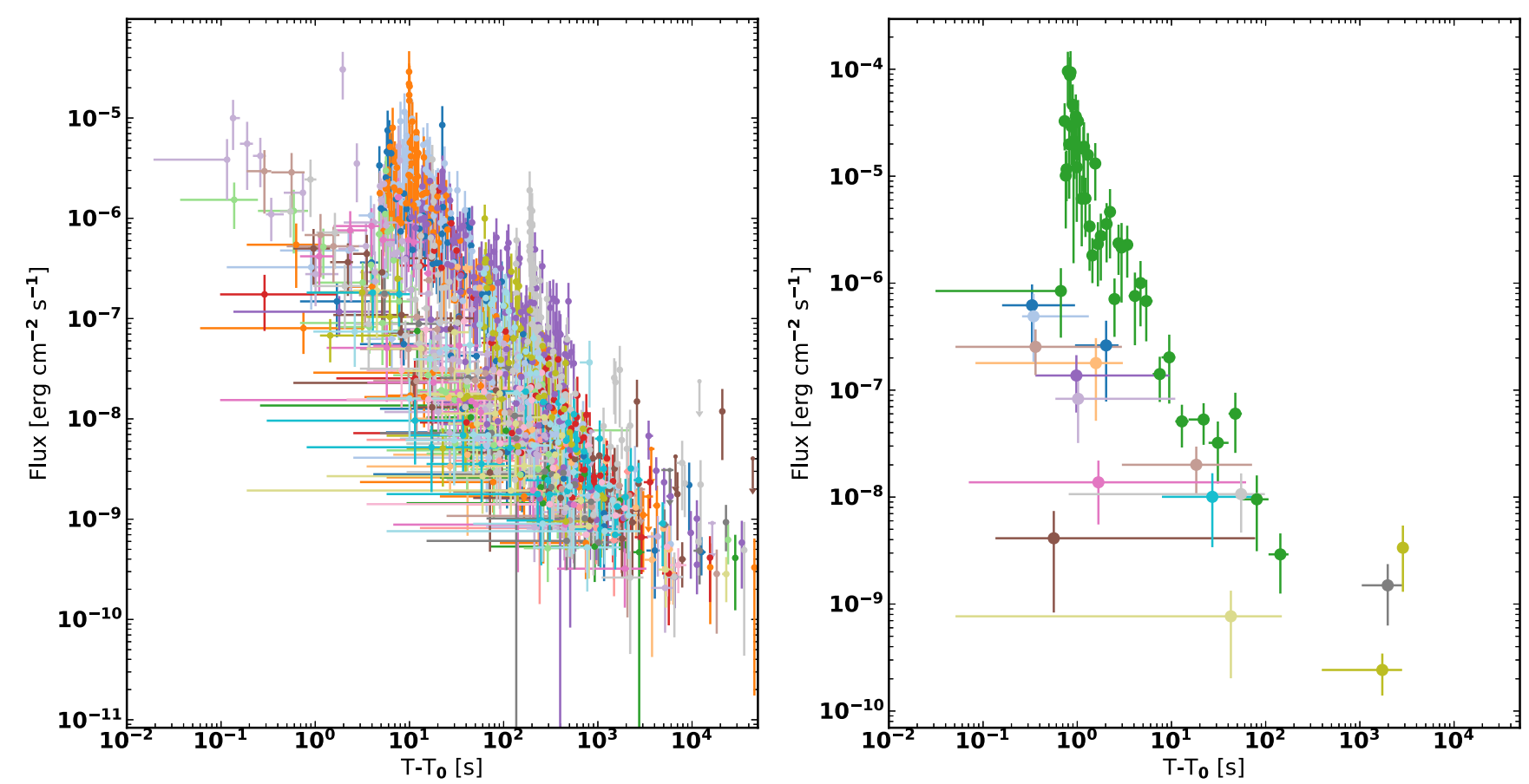

Figure 19. Flux calculated in the $100 \mathrm{MeV}-100 \mathrm{GeV}$ energy range vs. elapsed time after trigger for 115 long (left panel) and 11 short (right panel) bursts. Each color represents a separate GRB.

comparison, we also show a dashed line with decay index 10/7 (see further Section 5.6).

\subsection{High-energy Events}

The highest-energy GRB photon ever recorded by Fermi thus far is a $94.1 \mathrm{GeV}$ event connected with GRB 130427A (Ackermann et al. 2014). While displaying photon energies of a few hundred $\mathrm{MeV}$ is a common feature among the LATdetected GRBs, higher energies are relatively rare. Table 6 summarizes the highest-energy photon characteristics for each burst in our sample. It lists the total number of photons detected with probability $>90 \%$ of belonging to the burst, as well as the energy, arrival time, and probability of the highest-energy photon detected in the "GBM" time window. We also list the same quantities calculated in the time-resolved analysis.

Figure 24 shows the fraction of GRBs detected above selected energy thresholds $(250 \mathrm{MeV}, 500 \mathrm{MeV}, 1 \mathrm{GeV}, 5 \mathrm{GeV}, 10 \mathrm{GeV}$, $50 \mathrm{GeV}$ ). A sharp drop from $\sim 70 \%$ to $\sim 30 \%$ is seen at $5 \mathrm{GeV}$. There are three GRBs with emission above $50 \mathrm{GeV}(2 \%)$, namely, GRB 130427A (95 GeV), GRB 140928A (52 GeV), and GRB 160509A (52 GeV).

Our sample of 34 GRBs with measured redshift also allows us to study the source-frame-corrected energies. This is shown as the dashed line in Figure 24. This distribution shows a more gradual decrease with energy: almost $80 \%$ of the included GRBs have a maximum source-frame photon energy above $5 \mathrm{GeV}$, and $\sim 12 \%$ (four GRBs) above $100 \mathrm{GeV}$. The highest 

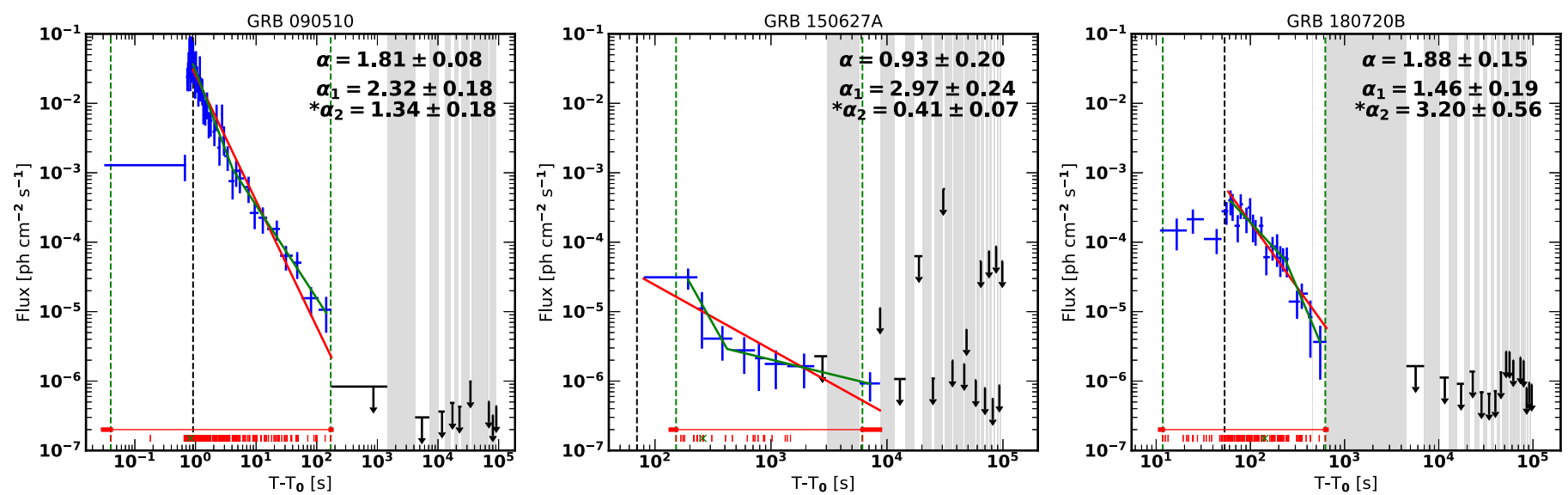

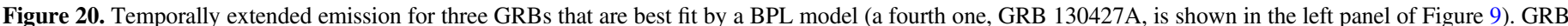

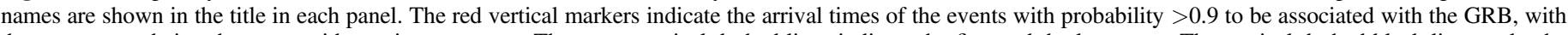

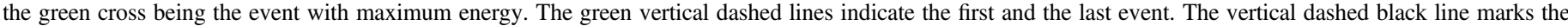

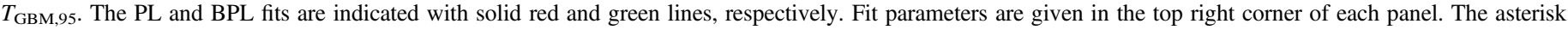
highlights the value selected as the temporal decay index in the analysis (which, in these cases, always corresponds to $\alpha_{2}$ of the BPL model).

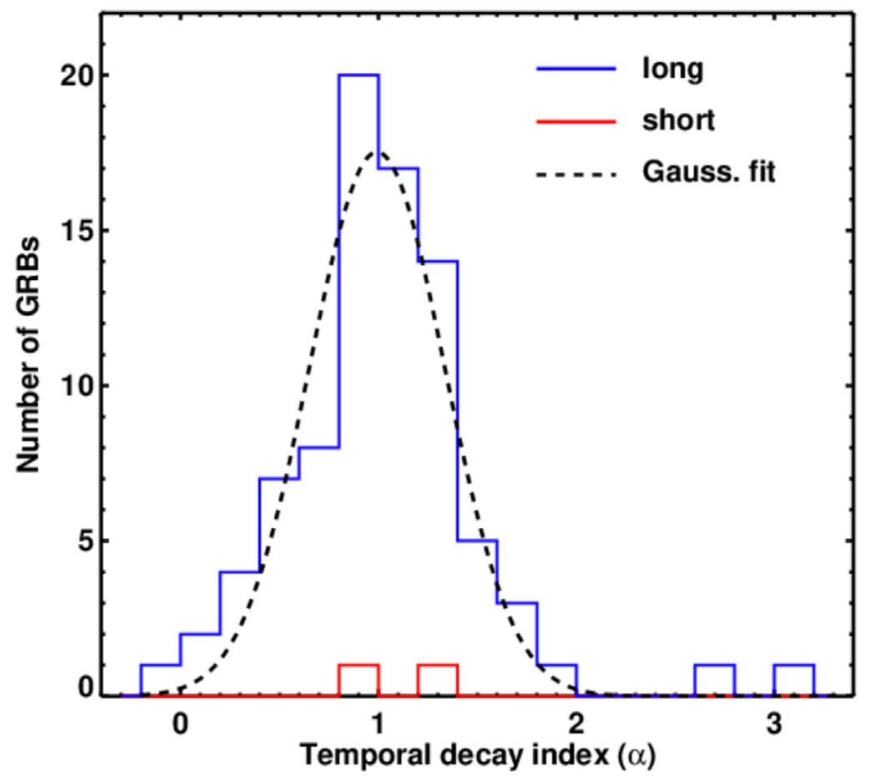

Figure 21. Distribution of the temporal decay indices for 86 long (blue histogram) and 2 short (red histogram) bursts, calculated in the "ETX" time window. A Gaussian fit of the lGRB indices (mean $0.99 \pm 0.04$; standard deviation $0.80 \pm 0.07$ ) is superimposed on the distributions (dashed black line).

source-frame energy is a $147 \mathrm{GeV}$ photon from GRB $080916 \mathrm{C}$, at $z=4.35$ (Atwood et al. 2013b). The figure also includes a linear fit to the bin centers of the source-frame distribution. The fit is remarkably good, showing that the fraction of GRBs decreases as $A \times \log (E / 1 \mathrm{MeV})+B$, where $A=-49 \pm 4$ and $B=266 \pm 21$.

Figure 25 shows the energy of the highest-energy photon in each GRB as a function of arrival time (left panel). In the right panel of the figure, the arrival time is shown as a fraction of $T_{\mathrm{GBM}, 90}$, calculated in the 50-300 keV range. No clear pattern can be distinguished, with long and short bursts overlapping in the right panel. The one clear outlier in the right panel is the short GRB 170127C, where the highest-energy photon $(500 \mathrm{MeV})$ was detected almost $3 \mathrm{ks}$ after the trigger. This GRB was outside the LAT FOV at $T_{0}$, and the data therefore only cover the time from around $300 \mathrm{~s}$ after the trigger (see also Section 4.2 and Figures 10 and 11).

In order to check for more high-energy photons to be detected beyond the standard energy range $(>100 \mathrm{GeV})$, we performed an additional analysis up to $300 \mathrm{GeV}$. No LAT detection was made above $100 \mathrm{GeV}$. Table 7 presents the 29 GRBs in the sample detected above $>10 \mathrm{GeV}$. All high-energy photons with a probability $>90 \%$ and with an observed energy $>10 \mathrm{GeV}$ are reported for each burst. As in Table 6, we specify the photon energy (listed in decreasing order), its arrival time, the GRB redshift, and the source-frame-corrected energy $\left(E_{\mathrm{sf}}\right)$. Values of $E_{\mathrm{sf}}>100 \mathrm{GeV}$ are marked in bold. The only short burst listed in this table is GRB 090510: in this case, a $\sim 30 \mathrm{GeV}$ photon was detected $830 \mathrm{~ms}$ after the GBM trigger time. GRB 130427A holds the record with 17 photons detected above $10 \mathrm{GeV}$, with the highest event ever detected from a GRB (the $94.1 \mathrm{GeV}$ photon) observed $243 \mathrm{~s}$ post-trigger. The second burst with the most HE photons is GRB 090902B, with seven photons detected above $10 \mathrm{GeV}$. There are two bursts where a high-energy photon is detected at very late times ( $>10 \mathrm{ks})$ : GRB 130427A ( 34 ks) and GRB 160623A $(\sim 12 \mathrm{ks})$.

\section{Discussion}

We will now discuss our results and compare them to previous results, in particular what was seen in the 1FLGC. The discussion generally follows the outline of Section 4, starting with the LAT sample as a whole, then continuing with a comparison to the GBM sample. Finally, we will consider the energetics, temporal decay, and possibilities for detections at very high energy (VHE). The aim of this section is to put our results in a wider context; broader implications in the framework of theoretical models will be discussed in Section 6.

\subsection{Detectability of LAT Bursts and LAT Detection Rate}

Before launch it was estimated that the LAT would detect 10-12 GRBs per year above $100 \mathrm{MeV}$ (Band et al. 2009). The results from the first GRB catalog showed $28 \mathrm{GRBs}$ detected in the first $3 \mathrm{yr}$ of the mission, slightly below expectations. The current work instead shows that the LAT has exceeded 

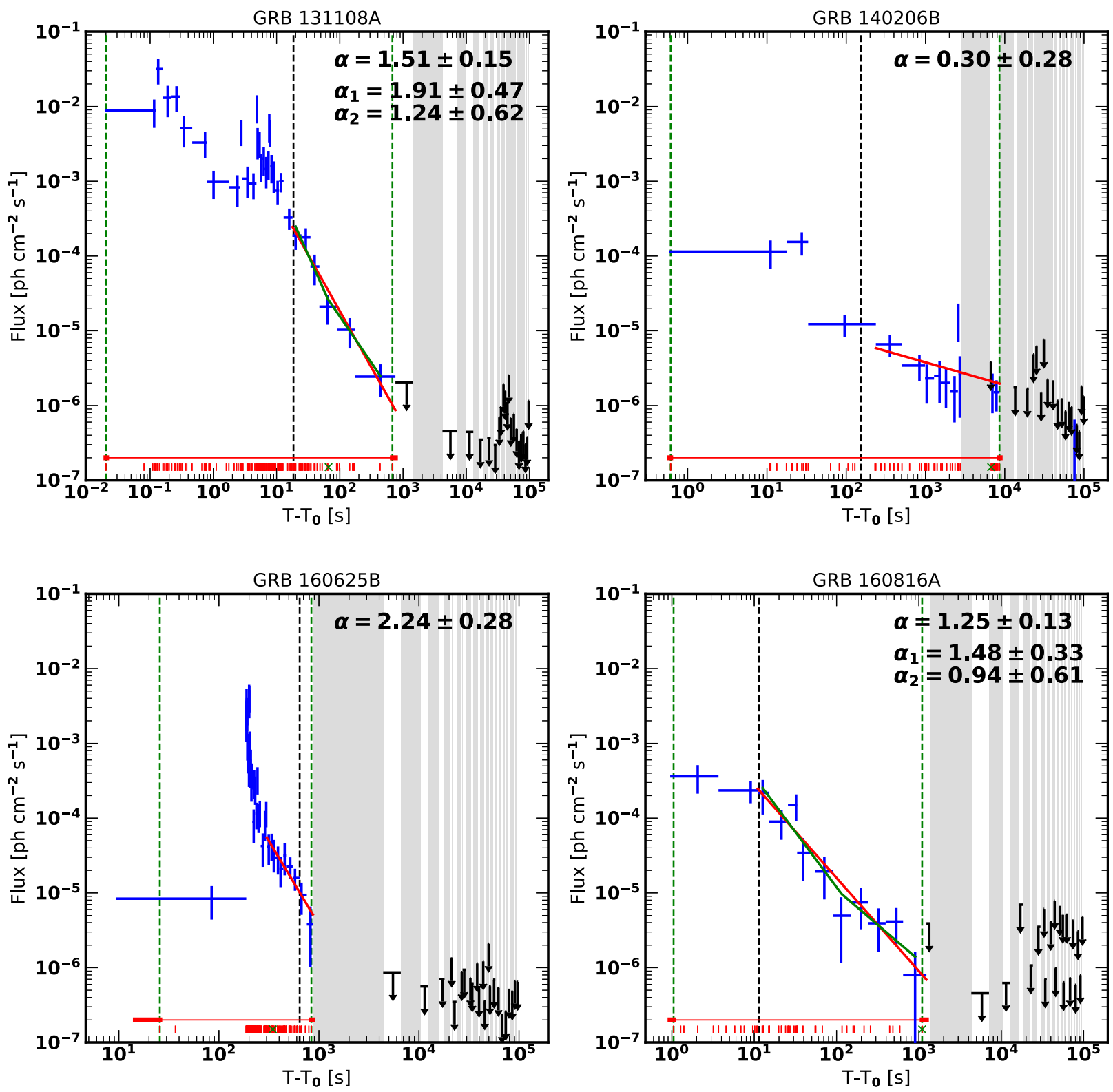

Figure 22. Temporal extended emission for four GRBs showing a peculiar behavior (the name of each GRB is shown in the title of each panel). Markers are the same as in Figure 20.

expectations, with 169 GRBs detected above $100 \mathrm{MeV}$ in $10 \mathrm{yr}$. This is in large part due to the continuous improvements in event analysis and detection algorithms.

It is interesting also to look specifically at the highest energies of the LAT-detected GRBs. As can be seen from Figure 24, only a small fraction of GRBs are seen in the upper energy range of the LAT. For example, $20 \%$ of the GRBs have detected emission above $10 \mathrm{GeV}$, corresponding to $\sim 3 \mathrm{GRBs}$ per year. We will further discuss the occurrence of these highest-energy events in Section 5.5.2.

Another interesting aspect to discuss is that while the highest-energy photon most often arrives after the $T_{\mathrm{GBM}, 90}$ (right panel of Figure 25), there is also a large fraction where it arrives during this interval. Interestingly, this fraction seems to vary slightly with energy: below $1 \mathrm{GeV}$ more than half of the events arrive during $T_{\mathrm{GBM}, 90}$, whereas $70 \%$ of events arrive later at energies above $10 \mathrm{GeV}$. We also note that there is little difference between the short and long GRB populations regarding normalized arrival time, although the maximum energy of sGRBs appears on average a bit lower. This could link the highest-energy photons to a process common in the two scenarios, and independent from the progenitor, such as external shocks in the circumburst medium. This will be further discussed in Section 6.

As of writing, the observing pattern adopted after the solar array malfunction (see Section 2.1) could potentially lead to a loss in GRB detections, as events occurring in the unobserved hemisphere of the sky may not be monitored until long after the event. However, studying the detections reported in this catalog, no such event was detected even during the previous observing strategy. The exception to this are the ARRs, as they could bring bright events into the FOV. It is thus likely that the detection rate will be slightly impacted while ARRs remain disabled. Even if the LAT detects late-time emission, without ARRs there is the risk of losing high-energy prompt emission. 

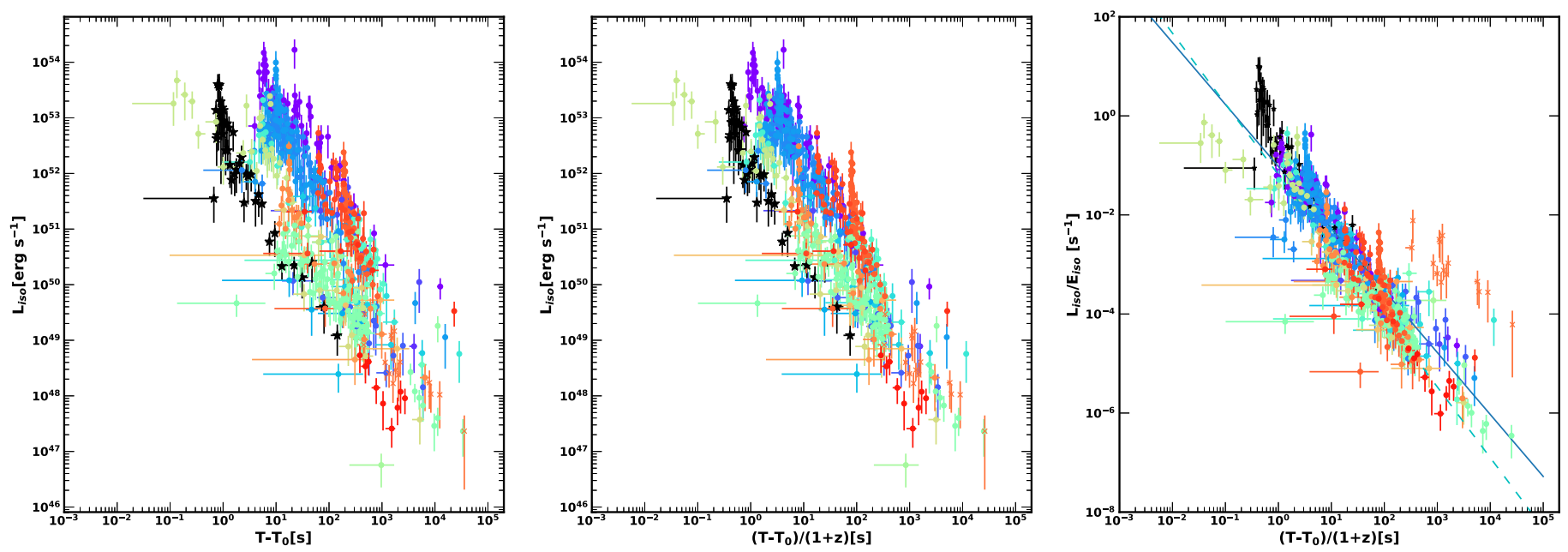

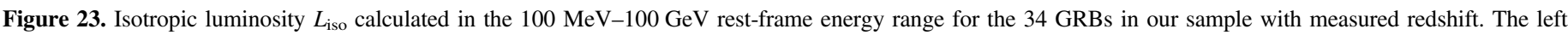

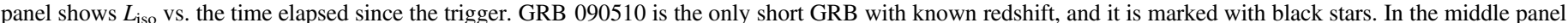

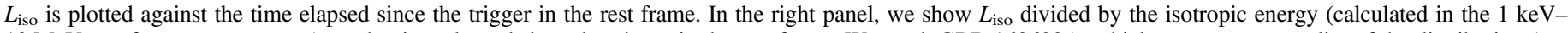

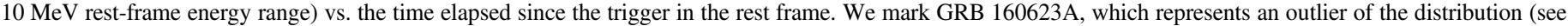

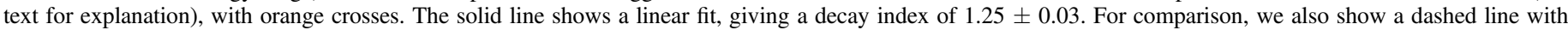
decay index $10 / 7$.

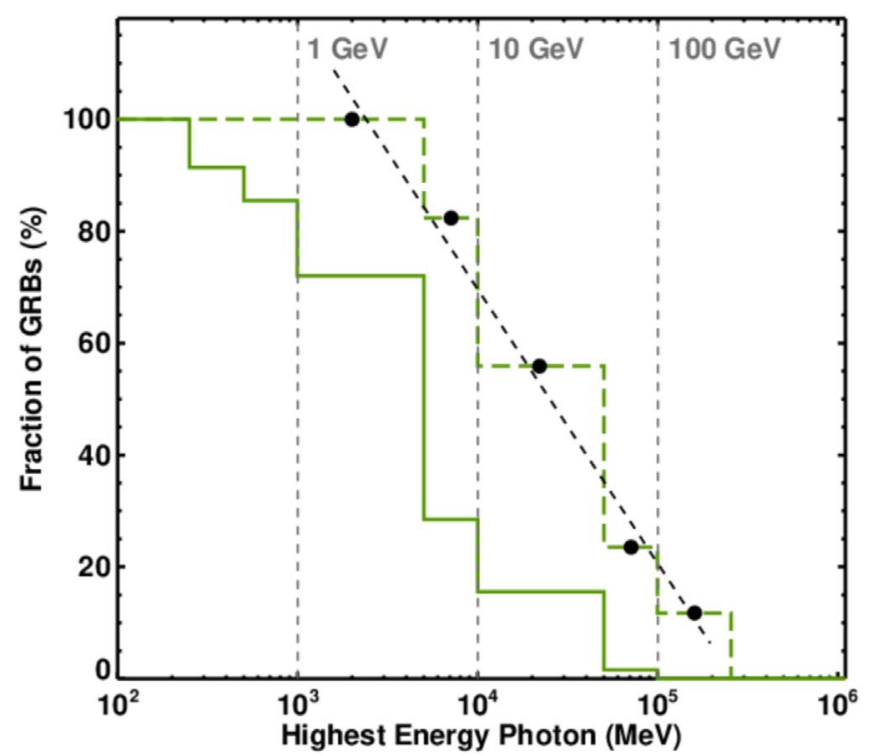

Figure 24. Fraction of GRBs with the highest-energy photon detected above selected threshold energies $(250 \mathrm{MeV}, 500 \mathrm{MeV}, 1 \mathrm{GeV}, 5 \mathrm{GeV}, 10 \mathrm{GeV}$, $50 \mathrm{GeV}$; green solid line). The distribution of the source-frame-corrected energies for the redshift sample is indicated with the dashed green line. The dashed black line denotes a linear fit to the values corresponding to the center of each bin.

\subsection{Onset and Duration of the High-energy Emission}

As in the 1FLGC, we can firmly establish the general trend that high-energy emission from GRBs tends to have delayed onset and longer duration as compared to emission at lower energies. However, Figure 7(c) also shows that when highenergy emission is detected, it starts during the prompt phase in $>60 \%$ of the cases. The majority of the other GRBs were outside the LAT FOV at trigger time, meaning that the fraction is likely even higher. This is an interesting result that provides valuable input to models of the emission mechanisms and will be discussed further in Section 6.
Figure 7 shows how varied the difference between $T_{\mathrm{GBM}, 05}$ (calculated in the $50-300 \mathrm{keV}$ range) and $T_{\mathrm{LAT}, 0}(100 \mathrm{MeV}-$ $10 \mathrm{GeV})$ can be. In some cases, the LAT emission is completely contemporaneous with the GBM. In other cases, the LAT emission starts hundreds or even thousands of seconds later. Considering only bursts that were in the LAT FOV at the time of trigger, there are a number of cases where the highenergy emission came much later than the one at lower energies. For example, GRB 160503A shown in the right panel of Figure 6 was at $\theta=25.1^{\circ}$ at $T_{\mathrm{LAT}, 0}$, and it remained in the FOV for over $2 \mathrm{ks}$ without any high-energy emission being seen. The first detection instead came much later, at $5 \mathrm{ks}$. These extreme delays are much longer than the ones seen in the 1FLGC and represent a new result in the 2FLGC.

In addition to greater delays, we now report much longer durations. In the 1FLGC, the longest duration reported was $>800 \mathrm{~s}$ for GRB 090902B. In the 2FLGC, many GRBs have durations of order $10^{3} \mathrm{~s}$, with the longest duration being $35 \mathrm{ks}$ (GRB 160623A). In general, the durations have increased also for most bursts contained in the 1FLGC, likely due to better sensitivity as a result of Pass 8 (we note, however, that the duration estimates were made using a different technique as described in Section 3.6.2, so the numbers are not directly comparable).

\subsection{Comparison with GBM Flux and Fluence Distribution}

As already presented in Section 4, the LAT-detected GRBs tend to sample the upper range of the GBM flux and fluence distribution (Figure 13). At the high end of the GBM fluence distribution, the LAT detects a high fraction of the GRBs; above $\sim 10^{-4} \mathrm{erg} \mathrm{cm}^{-2}$ the two distributions practically overlap (left panel). The few additional bursts seen by the GBM could be explained by some GRBs being outside the LAT FOV or at high zenith angles. The effect of the $\theta$ angle is further investigated in Ajello et al. (2018), who show that this is the main factor determining LAT detectability. The bias toward high GBM flux and fluence for LAT-detected bursts is therefore not related to a difference in sensitivity between the instruments. 

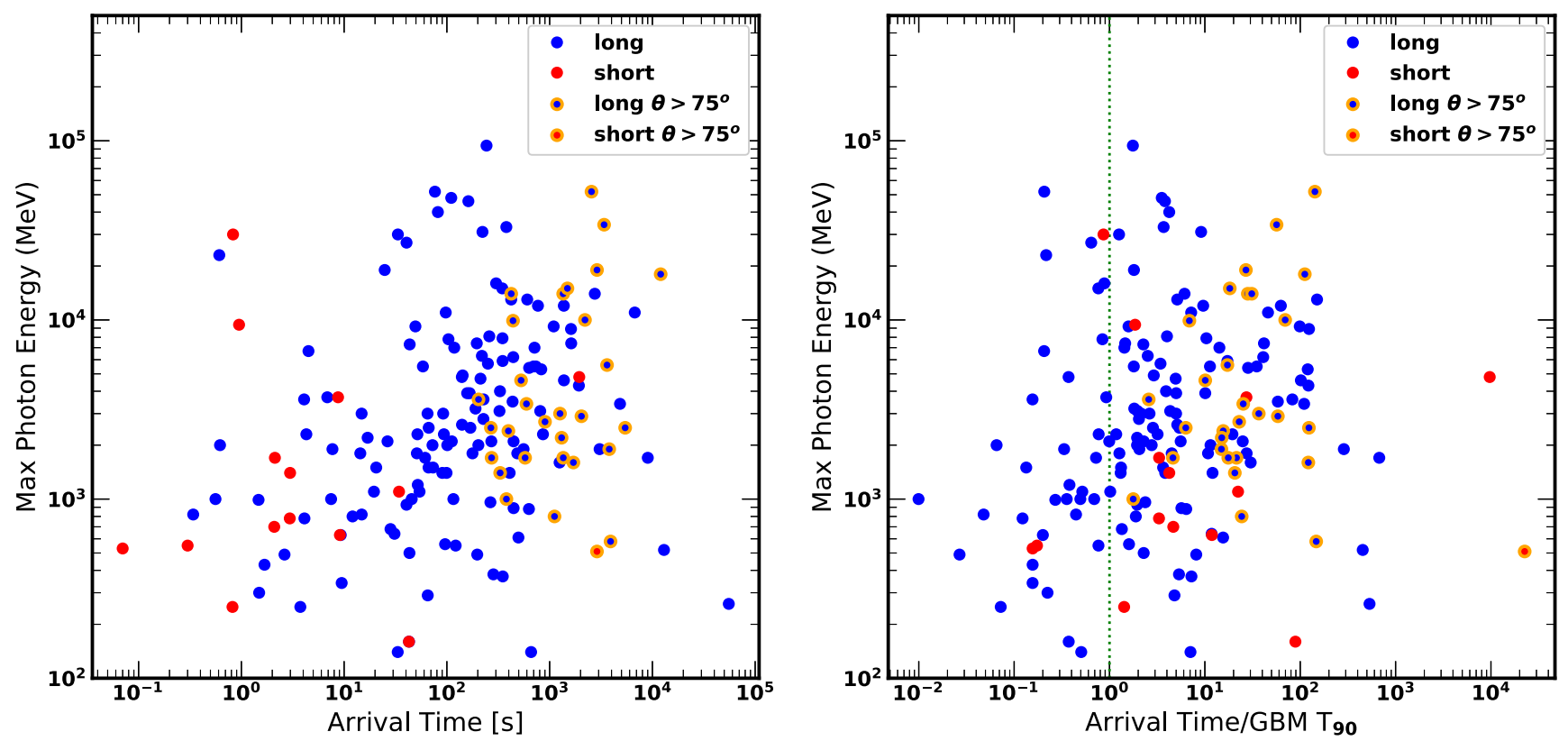

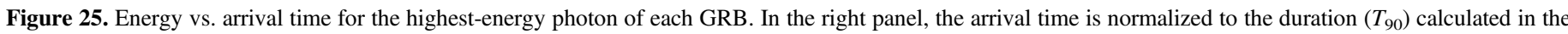
50-300 keV energy range (indicated by the dashed vertical line). Blue and red circles represent long and short GRBs, respectively.

Looking at the high end of the flux distribution (middle panel of Figure 13), the fraction of GRBs not seen by the LAT is higher. This shows that the flux might not be such a good indicator for high-energy emission. While the fluence is a measure of the total energy output, the flux simply shows the "strength" of the peak. The light curves also show clear differences, and peaks at low energies are not necessarily mirrored at high energies. An example of this is GRB $180728 \mathrm{~A}$, where the GBM peak flux was about 230 photons $\mathrm{cm}^{-2} \mathrm{~s}^{-1}$ (Veres et al. 2018), but no detection was made at high energy even though the burst was inside the LAT FOV $\left(\theta \sim 35^{\circ}\right)$. This is likely due to the low value of $E_{\text {peak }}$, $80 \mathrm{keV}$; indeed, the flux was dominated by energies below $50 \mathrm{keV}$.

However, the LAT has also detected GRBs that have relatively low fluence in the GBM. These outliers are predominantly short GRBs, where the low fluence is naturally explained by the short duration. The fluence distribution of sGRBs in the GBM is also shifted to lower values overall. Also for sGRBs there is a tendency for the LAT to sample the higher fluence end of the GBM population, but this is much less marked than for the lGRBs.

The result that the LAT detections are biased toward the brighter GRBs was clear already in the 1FLGC. However, with the larger sample, the picture presented here becomes more nuanced. The sGRBs show that high-energy emission can be produced even at lower fluence, raising the question of why not more lGRBs are detected. There may be differences in the emission mechanisms or environments between the classes that explain why low-fluence sGRBs are more likely to be detected in high-energy emission than lGRBs of similar fluence. For instance, lGRBs are expected to have a denser circumburst medium, as they are coupled to massive stars that have strong stellar winds.

The possibility of $\mathrm{GeV}$ emission from sGRBs is particularly interesting in the light of GW170817 and the associated sGRB 170817A (Abbott et al. 2017a, 2017b; Goldstein et al. 2017). This event was outside the LAT FOV and had a fairly low fluence of $2.8 \times 10^{-7} \mathrm{erg} \mathrm{cm}^{-2}$. However, Figure 13 shows that the LAT has detected GRBs with a similar fluence. This is very promising in view of the upcoming observation period scheduled to start in spring 2019, showing a strong potential for LAT detections of similar events (see also Ajello et al. 2018).

\subsection{Origin of Emission below $100 \mathrm{MeV}$}

In our sample almost twice as many GRBs are detected above $100 \mathrm{MeV}$ (169) as in the 30-100 MeV LLE range (91). Several studies have found evidence for a separate spectral component behind the emission above $100 \mathrm{MeV}$, and highenergy cutoffs between the LLE and LAT energy ranges have also been seen (e.g., Vianello et al. 2018). The behavior of the emission below $100 \mathrm{MeV}$ further shows more similar temporal behavior to the GBM range than do the data above $100 \mathrm{MeV}$ (see Figures 7 and 12). The LLE-only GRBs in our sample would then be the result of the low-energy emission being strong enough (and/or $E_{\text {peak }}$ at high enough energy) to extend into the LLE range. As the burst evolves, $E_{\text {peak }}$ moves to lower energies, and the emission in the LLE range will therefore appear to fade before that at lower energies. This explains the fact that the duration at $30-100 \mathrm{MeV}$ is almost always shorter than the one measured by the GBM. This picture also explains the fact that the LLE emission appears to start earlier than the GBM emission in a few cases (left panel in Figure 12). As the duration is shorter in the LLE range, this will naturally make the value of $T_{\text {LLE.05 }}$ shorter as well. Again, it is just a sign that the emission in the GBM range lasts much longer than the one in the LLE range.

For GRBs detected in both LAT and LLE energy ranges, the duration is generally shorter in the $30-100 \mathrm{MeV}$ band. While direct comparison of the $T_{\mathrm{LLE}, 90}$ and $T_{\mathrm{LAT}, 100}$ should be done with caution, the large differences in duration here clearly point to an intrinsic origin rather than observational bias. For example, in the LLE range the effective area is up to a factor of 2-3 lower than that above $1 \mathrm{GeV}$; however, the $T_{\mathrm{LAT}, 100}$ can be more than an order of magnitude longer than the $T_{\mathrm{LLE}, 90}$. 
Although there is much to suggest that the LAT emission is often the result of a separate component, we caution that there are also cases where the GRB spectrum is seen in the full range from $\mathrm{keV}$ to $\mathrm{GeV}$ and well fit by a single component extending also to energies above $100 \mathrm{MeV}$ (e.g., Abdo et al. 2009c; Axelsson et al. 2012). So while the dominance of LAT-only detections supports a separate emission process $>100 \mathrm{MeV}$ in general, proper spectral analysis must be made to draw conclusions about an individual burst.

\subsection{Energetics in the Prompt and Afterglow Phases}

We first investigate the energy output in different time windows. Figure 14 allows us to qualitatively compare the energy output during the total duration of each GRB as measured in the 50-300 keV (GBM) and $100 \mathrm{MeV}-10 \mathrm{GeV}$ (LAT) ranges. In the "GBM" duration window, the flux (top left panel) is inversely proportional to the duration. A possible interpretation is that a limited amount of total energy is available for any given GRB. A longer duration then means that the average flux will decrease. Comparing the fluences (top right panel), long and short GRBs are clearly separated, with all but one of the sGRBs having fluence below $10^{-6} \mathrm{erg} \mathrm{cm}^{-2}$. Within each group, fluence is independent of duration, suggesting that the separation arises as a result of different energy budgets, as might be expected if the groups arise from two different progenitor scenarios.

In contrast, long and short GRBs show no clear separation in the "LAT" duration window (bottom panels). While the populations occupy slightly different regions of the plots, there is a smooth transition between them. This may indicate that the emission in this window is dominated by the afterglow and that this process is similar regardless of progenitor. In the right panel, the fluence shows a tendency to increase with duration. This could have many explanations: a variable energy budget, meaning that more energetic afterglows last longer; an effect of differences in the circumburst medium, leading to varying radiative efficiency; or variations in the viewing angle with respect to the jet. We caution that there is likely also an observational bias: GRBs with favorable conditions can be studied longer; these will then have both a higher number of detected photons (i.e., greater fluence) and a longer measured duration.

Looking closer at the energetics, we can compare the LAT $100 \mathrm{MeV}-10 \mathrm{GeV}$ fluence to that of the $10 \mathrm{keV}-1 \mathrm{MeV}$ range in the GBM. We make this comparison in the "GBM" time window (left panel of Figure 15), which can be seen as a proxy for the prompt phase of the burst. We again stress that no joint spectral fits have been made and that the fluences are calculated based on different spectral fits in each range: the GBM fits are taken from the FGGC, while a PL fit is used for the LAT range (see Section 3.6). In the 1FLGC, it was found that the highenergy fluences from the joint GBM-LAT spectral fit agreed with those from LAT-only analysis, with a small discrepancy seen only for the brightest GRBs. Therefore, we do not expect any significant bias in our results, but a more thorough study needs to be performed before detailed conclusions can be drawn. Such analyses will be presented in future publications.

As found in the 1FLGC, the bulk of the population emits most of its radiation in the GBM energy range, with the highenergy emission reaching $\lesssim 20 \%$ of that in the lower band. However, given the larger sample in the 2FLGC, it is clear that this mainly applies to long GRBs. The short GRBs are more clustered around the line of equality, and several have higher fluence in the $100 \mathrm{MeV}-10 \mathrm{GeV}$ range than in the $10 \mathrm{keV}-$ $1 \mathrm{MeV}$ one. We therefore conclude that short GRBs in the prompt phase tend to emit a relatively larger fraction of their energy at higher energies, as compared to long GRBs.

In the next step we compare the relative energy output in the $100 \mathrm{MeV}-10 \mathrm{GeV}$ range during the "GBM" (prompt) and "EXT" (afterglow) time windows of each burst (right panel of Figure 15). Similar to the results found in the 1FLGC, most GRBs are clustered around the line of equality, meaning that in the LAT energy range comparable amounts of energy are released in the prompt and afterglow phases. For long GRBs there is a large fraction where the fluence during the afterglow phase is greater than that during the prompt phase. The short GRBs instead tend to have equal fluence in the two time windows.

In the 1FLGC, there appeared to be a correlation between the photon index and GBM duration, with shorter bursts tending to have harder spectra. This was coupled to the general property that short GRBs on average have harder spectra. Figure 16 shows that this overall trend is not held up in the current larger sample. Furthermore, although the short GRBs on average have slightly harder spectra in the "GBM" time window (top left panel), this difference is not seen in the "EXT" window (top right panel). This could merely be an indication that the GRB spectra are sometimes "contaminated" by emission from lower energies, i.e., the $\beta$ value of the Band function. This value is typically just below -2 , and the distribution has a long tail to lower values (Gruber et al. 2014). The larger number of lGRBs means that we are more likely to see the effect in this population. Comparing the spectral index between the "GBM" and "EXT" windows, the small difference $(\Delta \Gamma \sim 0.5)$ might indicate that we are seeing the early afterglow already in the "GBM" window. This possibility will be discussed further in Section 6.

The bottom left panel of Figure 16 shows that the spectral index is not dependent on flux in the "GBM" window. Instead, the bottom right panel would appear to suggest a correlation between flux and photon index in the "EXT" time window, such that stronger emission is coupled to softer spectra. This is especially obvious in bursts with lower flux. However, we caution that this is likely an observational bias. Weaker bursts will only be detected if they have harder spectra.

\subsubsection{Intrinsic Energetics}

In the 1FLGC, there were 10 LAT-detected GRBs with measured redshift. In the $2 \mathrm{FLGC}$, the number has increased to 34, allowing us to better study their intrinsic properties. Already Figure 13 suggests that the LAT GRBs are among the brightest. Comparing with the Swift and GBM samples in Figure 18, it is clear that this is an intrinsic property and that the LAT preferentially detects brighter GRBs, regardless of redshift. The two panels in Figure 18 show that in order for the LAT to detect a GRB at high redshift, such a burst must be intrinsically very bright. For example, all detected GRBs at $z>1$ have $E_{\text {iso }}>10^{52} \mathrm{erg}$, while detecting a GRB at $z>4$ requires $E_{\text {iso }}>10^{54} \mathrm{erg}$. After $10 \mathrm{yr}$, GRB080916C is still the most distant GRB observed by the LAT, at $z=4.35$. 

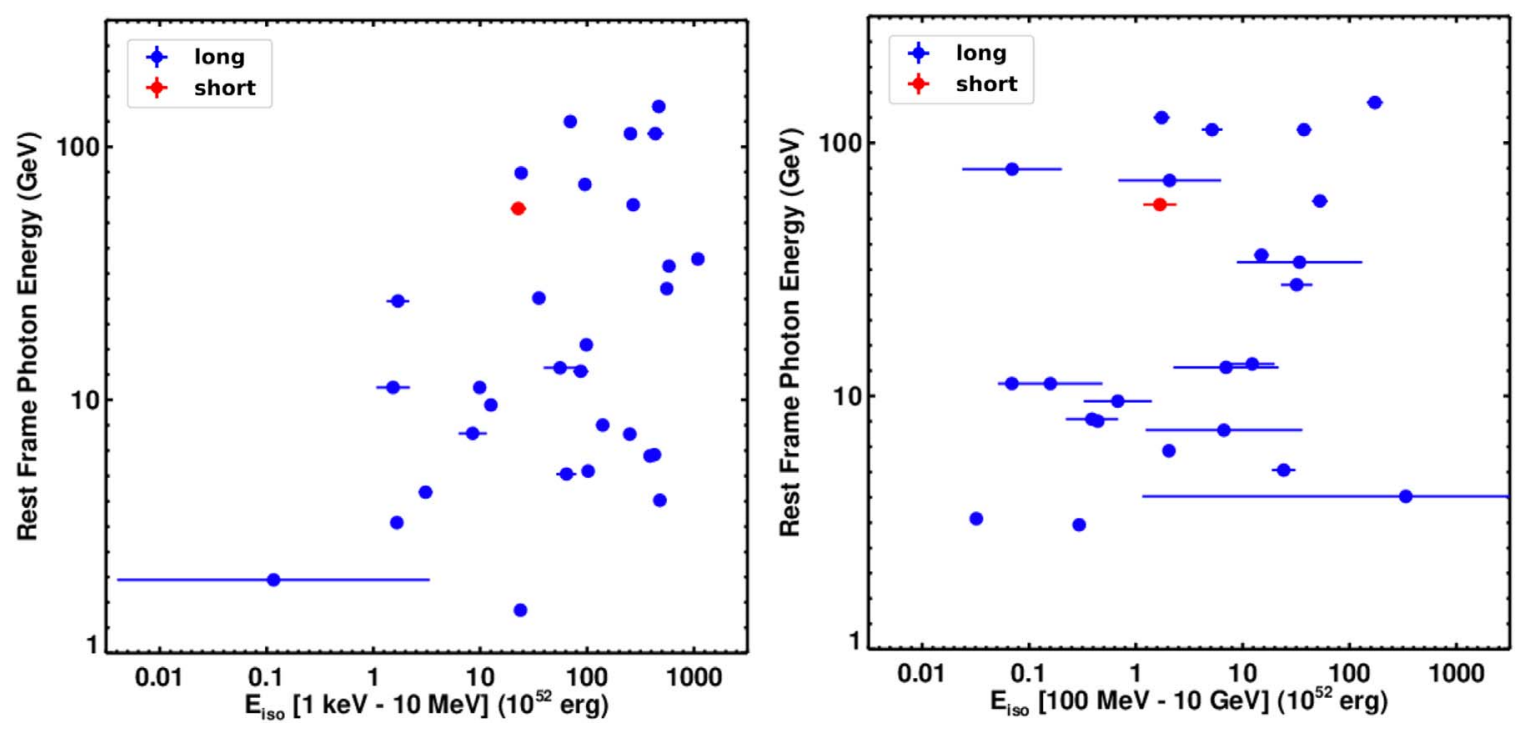

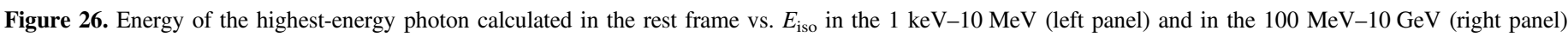
energy ranges for each GRB with redshift estimation. Blue and red circles represent long and short GRBs, respectively.

\subsubsection{The Highest-energy Photons}

We now turn to the highest-energy photons detected by the LAT, as presented in Figure 24. Although less than $80 \%$ of GRBs do not reach above $5 \mathrm{GeV}$ in the observer frame, the figure indicates that higher energies are more common in the source frame, with $\sim 15 \%$ of GRBs reaching source-framecorrected energies above $100 \mathrm{GeV}$. We note, however, that as there is only one sGRB with measured redshift, the rest-frame distribution is dominated by lGRBs. Interestingly, the fraction of GRBs detected drops smoothly as the threshold energy in the rest frame increases. It is tempting to connect this behavior to the underlying spectral distribution, such that we are seeing the limit determined by the intrinsic spectral shape, which seems to be similar for all GRBs (see Figure 16). In bright bursts more high-energy emission is produced, allowing $\mathrm{GeV}$ emission to be observed. Faint bursts will produce too little $\mathrm{GeV}$ emission, and the LAT will only detect MeV photons.

Although it is clear that the high-energy emission in general can last a long time, the highest-energy photon in a given GRB in some cases arrives relatively soon after the trigger. For example, this photon arrives within $<2$ s from the trigger time for $\sim 50 \%$ of the sGRBs in the left panel of Figure 25 . The right panel of Figure 25 also shows that among the GRBs with a maximum photon energy $>10 \mathrm{GeV}$, the highest-energy photon is in some cases detected before the emission in the $50-300 \mathrm{keV}$ energy range is over. Particle acceleration in GRBs must be efficient in order to produce such high-energy gamma-rays within such a short time. Considering internal opacity of the jet outflow also leads us to conclude that a high bulk Lorentz factor is necessary in order for us to be able to detect these photons. Indeed, these considerations have been used to estimate a Lorentz factor well above 500 in several LAT-detected bursts (see, e.g., Abdo et al. 2009a, 2009c).

In the 1FLGC, there was a trend for the photons with highest source-frame energy to appear in the GRBs with highest $1 \mathrm{keV}-$ $10 \mathrm{MeV} E_{\text {iso }}$ (Figure 21 in the 1FLGC), and the one short GRB with redshift (GRB 090510) did not follow the pattern seen in long GRBs. However, these results were based on only nine GRBs. With the greater statistics in the 2FLGC, the trend not only persists but also is extended over a greater range, as shown in the left panel of Figure 26. This indicates that the maximum rest-frame energy produced is simply a function of the total energy output. The right panel instead shows the relation between the highest energy and $E_{\text {iso }}$ calculated during the "GBM" time window in the LAT energy range $(100 \mathrm{MeV}-100 \mathrm{GeV})$. In this range, the correlation is less obvious and the points are more scattered. As the energy output in the LAT range is much smaller than at lower energies (see the left panel in Figure 15), it is perhaps not surprising that the low-energy $E_{\text {iso }}$ is a more reliable estimator of the energy budget. However, there are a small number of GRBs that deviate from the general trend, showing low rest-frame energies despite a large $E_{\text {iso. }}$. More detailed studies are required in order to understand this behavior. In both panels the region of GRB 090510, which is still the only short GRB with a redshift measurement, is populated also by long GRBs. This again points to similar conditions for long and short GRBs. Finally, the high-energy gamma-rays from GRBs offer a valuable way to probe more physics than the burst itself. For example, the interaction of $>10 \mathrm{GeV}$ gamma-rays from sources at cosmological distance with optical and UV photons of the extragalactic background light (EBL) causes absorption of gammarays, modifying the high-energy part of the spectrum. Highredshift GRBs can thereby serve as a probe of EBL opacity (Desai et al. 2017). For example, EBL attenuation could contribute to the differences between the observer and rest-frame distributions in Figure 24.

\subsection{High-energy Emission Temporal Profile}

In the 1FLGC the study of the temporal decay of the extended emission led to the hypothesis that this is part of an early afterglow component very similar to the afterglow component seen in the X-ray band. The stable decay index with a value around $\alpha=1$ for the small sample of bursts in the 1FLGC was taken as support for adiabatic expansion within the fireball scenario. The index reported here remains centered around $\alpha=0.99$. If the extended component is fast-cooling afterglow emission from either a radiative or an adiabatic fireball in a constant-density environment, the decay indices (given a photon index of -2 ) would be $10 / 7$ or 1 , respectively (Katz \& Piran 1997; Sari 1997; Ghisellini et al. 2010). The 


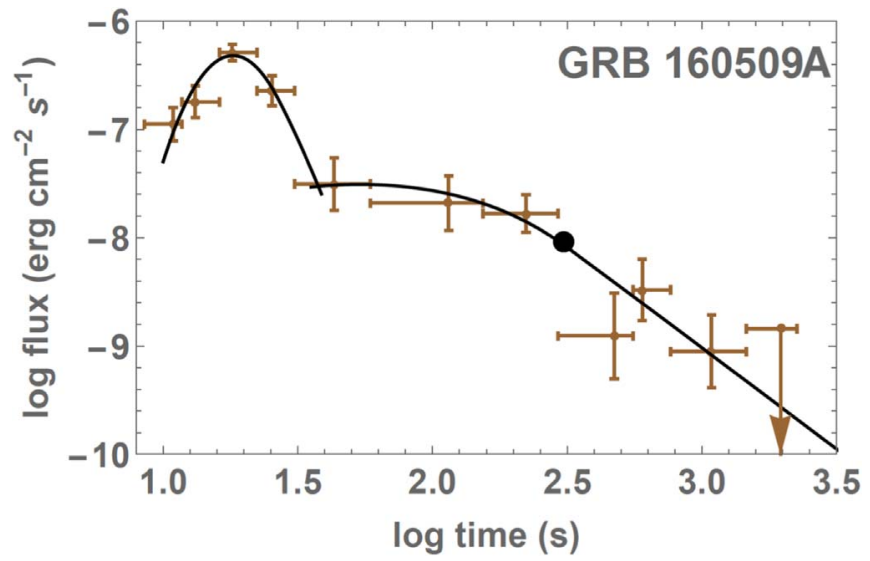

Figure 27. Light curve of GRB 160509A, together with the fit using the Willingale et al. (2007) model (solid line). The black circle indicates the end time of the plateau emission and its corresponding flux.

peak in the decay index distribution would thus primarily suggest the adiabatic scenario. Nevertheless, the range of measured indices allows also for a radiative fireball in at least a few individual bursts.

The luminosity distribution seen in Figure 23 shows that the GRBs with known redshift all have similar decay indices. Yet although the slope of the luminosity decay is similar among the GRBs, its distribution versus the elapsed time since the trigger time is scattered (left panel). In the middle panel, the time axis for each light curve has been corrected to the time in the rest frame, which reduces the spread. If the luminosity is divided by the isotropic energy of each burst, measured in the $1 \mathrm{keV}-10 \mathrm{MeV}$ range, the resulting light curves cluster even more (right panel in Figure 23), as reported by Ghisellini et al. (2010) and Nava et al. (2014). With higher statistics with respect to these studies (which were limited to a few LAT GRBs observed up until 2013), the clustering points to a strong correlation between the isotropic luminosity of the high-energy emission and the prompt isotropic energy calculated in the range $1 \mathrm{keV}-10 \mathrm{MeV}$.

In the analysis performed in Sections 3.6.2 and 4.7, some light curves are found to be best fit with a spectral break. Such features may be related to the X-ray afterglow plateaus, which are well established, but their nature is still debated. To investigate this, we analyze all LAT GRBs with TS $\geqslant 64$, with a sufficient number of photons. This choice of TS is dictated by the need of observing the $\gamma$-ray afterglow for a sufficiently long time to allow us to characterize the nature of the deviation from a PL. We then determine whether the light curves can be fitted within the phenomenological Willingale et al. (2007) model, used to fit the X-ray plateaus (the details of the analysis will be presented in M. Dainotti et al. 2019, in preparation).

We find that four GRBs (GRB 160509A, GRB 090902B, GRB 150627A, GRB 160816A) show a late-time flattening reminiscent of the X-ray plateaus (their light curves can be seen in Figures 20 and 22). The light curve of GRB 160509A, together with the Willingale et al. (2007) model, is presented in Figure 27. From this resemblance we infer that there is indication of the existence of a plateau in a few Fermi-LAT light curves. Our recovered values for $T_{\gamma}$, which is the time at the end of the plateau emission, are smaller than those typically found in the X-ray regime (from hundreds to a few thousand seconds; Willingale et al. 2007). This suggests that more energetic bursts have smaller plateau durations. Although a one-to-one comparison is difficult owing to the lack of corresponding X-ray observations for those bursts, it is nevertheless interesting to mention this trend for future reference and possible new observations.

\subsection{Prospects for GRB Detections at VHE}

The detection of VHE emission from GRB 190114C reported by the MAGIC Collaboration (Mirzoyan 2019) opened up a new era in the observations of GRBs. The bright source was seen across the electromagnetic spectrum, from radio to VHE. While studies of this burst are certain to yield interesting results, more VHE detections will be necessary to build an understanding of the mechanisms behind such emission.

The Cherenkov Telescope Array (CTA; Actis et al. 2011; The CTA Consortium et al. 2019) will mark an important milestone in the VHE range, covering the whole sky with unprecedented sensitivity. The CTA can steer to a GRB location in less than $30 \mathrm{~s}$ after receiving a trigger notice from a space-based instrument (see, e.g., Gilmore et al. 2013; Inoue et al. 2013). In an optimistic case, one can therefore assume that the CTA is on target within $\sim 50 \mathrm{~s}$ after the onset of the burst.

The GRB sample in the 2FLGC allows us to make a simple and phenomenological estimate of the detectability by VHE instruments. For this exercise, we use the observed LAT spectra as input for CTA simulations, considering only the 33 IGRBs with redshift measurements. We performed an ad hoc spectral analysis in a $2 \mathrm{hr}$ interval starting at $T_{\mathrm{LAT}, 0}+50 \mathrm{~s}$ in the energy range from $100 \mathrm{MeV}$ to $100 \mathrm{GeV}$. The LAT data are fit using a PL spectrum. The output spectral parameters (flux, $F_{0}$, and photon index, $\Gamma$ ) were then used as input for the CTA tools software package ctools, ${ }^{76}$ version 1.5 .2 , with the instrument response functions (IRFs) Prod $2^{77}$ referred to the CTA configuration in the Southern Hemisphere site. The CTA simulation requires as input the GRB location and spectral model and gives as output a TS map showing whether the source is detected or not. The extrapolation into the CTA range assumes either a PL or a cutoff PL (CUTPL) spectral model. The background is already taken into account in the used IRFs. We performed simulations over an energy range of $50 \mathrm{GeV}-$ $50 \mathrm{TeV}$, assuming that the CTA received the LAT detection notice with several different time delays: $0,0.5,1,2,4,6$, and $10 \mathrm{hr}$. We considered a $2 \mathrm{hr}$ CTA observational interval following the notice time, assuming a flux temporal decay of $F=F_{0} t^{-\alpha}$, where $\alpha$ is the temporal decay index taken from Table 4. For the $0 \mathrm{hr}$ delay we used all 33 GRBs, while for all the other analyses the number of cases was limited to the 27 GRBs where $\alpha$ could be constrained.

An important factor to consider in the VHE range $(>50 \mathrm{GeV})$ is that the gamma-ray spectrum can be attenuated by $\gamma \gamma$ absorption due to the annihilation of high-energy photons with photons from the EBL. In order to include the EBL attenuation in our simulation, the cutoff energy of the CUTPL model was chosen as the critical energy defined by Ackermann et al. (2011). For the GRBs at redshift $>1$ (20 GRBs), we fixed the critical energy at $30 \mathrm{GeV}$ (following Figure 2 of Ackermann et al. 2012b), while for the others at redshift $<1$ (13 GRBs) the

\footnotetext{
76 http://cta.irap.omp.eu/ctools/users/index.html

77 https://www.cta-observatory.org/science/cta-performance/
} 


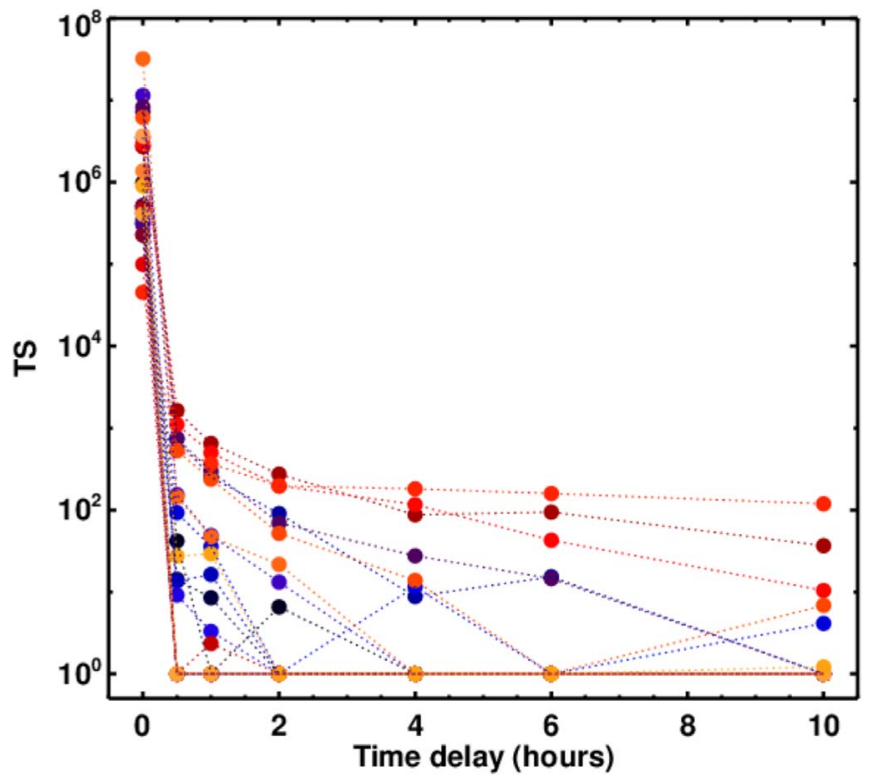

Figure 28. TS evolution with respect to the time delay (in hours) of the CTA alert reception, obtained from the analysis with a PL model, with respect to the beginning of the LAT-detected extended emission. The figure shows the TS values for 27 GRBs. For clarity, TS values less than 1 are fixed to 1 .

critical energy was chosen following Ackermann et al. (2012b): $E_{\text {crit }}(z)=170(1+z)^{-2.38} \mathrm{GeV}$. Spectral analyses of the CTA simulated data were then performed using both PL and CUTPL spectral models.

The analyses performed without any time delay $(0 \mathrm{hr})$ reveal that the CTA is able to detect all 33 GRBs with high TS, as shown in Figure 28 for the PL case. This shows that CTA telescopes would readily detect GRBs given ideal observational conditions during night time, i.e., assuming that the CTA was already on target or started observing immediately after a GRB trigger. However, the LAT data generally require more than $6 \mathrm{hr}$ to be downlinked and analyzed on the ground. Figure 28 also shows the TS evolution with respect to the time delay between the LAT detection and the CTA alert reception time in the case of a PL model. The TS values decrease rapidly with time, and given a $10 \mathrm{hr}$ delay from the LAT detection, the CTA can detect only two GRBs among 27 (with TS > 25). In the case of the CUTPL model, all GRBs are detected only in the most optimistic observing condition, and the TS values fall below 25 for all 27 GRBs already with a $0.5 \mathrm{hr}$ delay. These results show the strong need for rapid alerts and precise localizations to enable follow-up observations at VHEs.

We stress that the analysis presented here is the result of a very simplified scenario using a single spectral component. Extending this study to give more reliable estimates of the detectability with VHE instruments will require both more realistic models for the high-energy emission mechanisms in GRBs and using proper theoretical models of EBL.

\subsection{LAT sGRBs and Implications for $G W$ Observations}

The recent detection of GW170817 and the coincident GRB 1701817A clearly showed a coupling between merging neutron stars, gravitational waves, and short GRBs. This has made the rapid search for counterparts also in the $\mathrm{MeV}-\mathrm{GeV}$ range a high priority for the LAT. The LAT Collaboration has previously reported on follow-up studies of several GW triggers: GW150914 (Ackermann et al. 2016), LVT 151012 and GW151226 (Racusin et al. 2017), and GW170817 (Ajello et al. 2018). The first three triggers were associated with mergers of two black holes, so no electromagnetic counterpart was expected. In the last case, the location could only be observed by the LAT starting $1100 \mathrm{~s}$ after the GBM trigger time, and again no detection was made. Nevertheless, these events have shown the importance of a dedicated pipeline to search for high-energy counterparts for a larger class of transient events than just GRBs. The LTF pipeline described in Section 3.2 has formed the baseline for these searches. In addition, dedicated searches have been developed to cover various time intervals (also very long ones) around the GW triggers, which are thoroughly described in Vianello et al. (2017). A particular challenge is the large area given by the LIGO probability map (several hundred square degrees), much larger than for a typical GRB.

As already said, due to observational constraints, the LAT could observe the location of GW170817/GRB 170817A only after more than $1 \mathrm{ks}$. In the sample of 14 sGRBs presented here, most events are only detected up to a few seconds after the GBM trigger. However, GRB 160702A was not seen until $\sim 1900 \mathrm{~s}$ after the trigger and lasted for about $300 \mathrm{~s}$. Also GRB $170127 \mathrm{C}$ has an unusually long duration: from $\sim 650$ to $\sim 2900$ s after the trigger. Neither of these events was especially bright (flux $<10^{-5} \mathrm{erg} \mathrm{cm}^{-2} \mathrm{~s}^{-1}$ ), showing that the LAT has the potential to find counterparts even to GW events that occur outside the FOV. Overall, the LAT has found high-energy counterparts to $5 \%$ of the sGRBs seen by the GBM. A critical discriminating factor that has been carefully addressed by Ajello et al. (2018) is the fact that the LAT can detect fainter sGRBs the earlier the observation starts, ideally within 100-200 s post-trigger. They calculated that the LAT can observe (either a detection or upper bound) $\sim 23 \%$ of the fullsky sGRB population within $100 \mathrm{~s}$ from their GBM trigger. Moreover, assuming that the LAT will have the same efficiency for GRB/GW triggers and a rate of joint GBM/GW events of 1 (2) per year, they obtained a $\sim 5 \%(\sim 10 \%)$ probability of detecting one or more GRB/GW events with the LAT in $1 \mathrm{yr}$, respectively. This was derived assuming that GRB/GW events will be representative of the entire GBM-detected sGRB population when observed in gamma-rays. Indeed, two other important factors affecting the detectability of such events by the LAT might be the off-axis viewing angle and the GRB distance.

Recently, von Kienlin et al. (2019) presented a search for GRBs in the entire 10 yr Fermi-GBM sample that show similar characteristics to GRB 170817A, i.e., a nonthermal pulse followed by a thermal component. They identified 13 candidates and concluded that the observed similarities likely arose as a result of the same processes that shaped the gammaray signal of GRB 170817A. We examined their sGRB sample and found that GRB 120915A has also been detected by the LAT and is included in the 2FLGC. The short duration in the LAT $\left(T_{\mathrm{LAT}, 100}=0.6 \pm 0.3 \mathrm{~s}\right)$ clearly ties the high-energy emission to the prompt phase. This again shows that the LAT is likely to detect such events as long as they are in the FOV at the time of the GW trigger, as in the case of GRB 120915A (with $\theta=11^{\circ}$ ).

\section{Theoretical Implications}

Observations of GRBs with Fermi-LAT have significantly broadened our understanding of the nature of high-energy 
emission from these powerful transients and have led to renewed theoretical activities to model this emission (see, e.g., Gehrels \& Razzaque 2013, for a review). Already the 1FLGC hinted at several new prominent characteristics of the highenergy emission: delayed onset compared to the GBM trigger; a hard spectrum, often requiring an additional component when modeling together with GBM data in the prompt phase; variable emission during the early prompt phase; and temporally extended emission beyond the prompt phase without measurable short-timescale variation. Although no joint GBM-LAT spectral fits have been performed for the 2FLGC, with its greatly enhanced sample the results presented here have firmly established at least the temporal properties as being general to the population as a whole. Modeling these characteristics gives us clues to the particle acceleration sites, environment, and radiation processes, while measurements of fluence, redshift, and energetics help us to classify the LATdetected GRBs in the context of the broader GRB population.

Simultaneous multiwavelength observations of a handful of LAT-detected GRBs, e.g., GRB 090510 (Ackermann et al. 2010a), GRB 110731A (Ackermann et al. 2013a), and GRB 130427A (Ackermann et al. 2014; Maselli et al. 2014), have further helped constrain the origin of a few high-energy emission features, such as the temporally extended emission. Regardless of the origin of high-energy emission, merely the fact that we detect it implies that the bulk Lorentz factor of the GRB fireball (Lithwick \& Sari 2001; Granot et al. 2008; Hascoët et al. 2012) is large. In particular, the fireball can be completely transparent to high-energy emission for sufficiently large bulk Lorentz factors (Razzaque et al. 2004). Furthermore, detection of $\gtrsim 10 \mathrm{GeV}$ photons from GRBs has constrained models of the extragalactic background light (Abdo et al. $2010 \mathrm{~b}$ ) and the models leading to violation of Lorentz invariance (Abdo et al. 2009b). Finally, the detection of the short GRB 170817A by Fermi-GBM (Goldstein et al. 2017) in coincidence with the gravitational wave event GW170817 (Abbott et al. 2017a, 2017b, 2017c) has proven that at least some short GRBs originate from mergers of binary neutron stars.

The search for the origin of $\mathrm{GeV}$ emission from GRBs has intensified since Fermi was launched and the LAT has detected an increasing number of GRBs. The results presented here make these efforts even more promising, as the large sample of the 2FLGC shows that high-energy emission can be seen also from some fainter GRBs (see Figure 13). In the following, we will examine how $\mathrm{GeV}$ emission from GRBs can be used to test theoretical predictions. These examples are not exhaustiverather, they are meant to illustrate various ways in which LAT observations can inform theoretical models.

\subsection{Delayed Onset and Long Duration: Challenges for Emission Models}

Synchrotron self-Compton (SSC) models for $\mathrm{GeV}$ emission are a natural extension of the synchrotron model for keV-MeV emission from GRBs, in the context of the internal shock scenario (see, e.g., Bošnjak et al. 2009; Wang et al. 2009; Ackermann et al. 2010a). However, in a simple SSC model the Compton emission can be delayed compared to synchrotron emission by at most the flux variability timescale $(\lesssim 1 \mathrm{~s}$ for long GRBs). Such models therefore cannot account for the $\gg 1 \mathrm{~s}$ delay observed in a large fraction of LAT GRBs (see Figure 7). $\mathrm{SSC}$ emission from late internal shocks, with much longer variability time at $\mathrm{GeV}$ than in $\mathrm{MeV}$, can explain delayed LAT onset in some cases (see, e.g., Toma et al. 2009; Daigne et al. 2011).

A possible alternative for $\mathrm{GeV}$ emission during the prompt phase is hadronic emission, either proton-synchrotron radiation and radiation from $e^{ \pm}$pair cascades (Razzaque et al. 2010) or photohadronic interactions and associated cascade radiation (Asano et al. 2009). In contrast to many SSC models, hadronic models can account for delayed onset of $\mathrm{GeV}$ emission via a hard PL component with its flux increasing over time owing to proton acceleration and cooling of cascade particles. Hadronic models, however, require $\gtrsim 100$ times more energy in the hadrons than in leptons, which is challenging to produce in GRBs. Furthermore, neither SSC nor hadronic emission mechanisms active during the prompt phase are able to explain the extended $\mathrm{GeV}$ emission beyond the keV-MeV $T_{\mathrm{GBM}, 90}$.

The delayed onset, smooth light curve, and temporally extended emission $\gtrsim 100 \mathrm{MeV}$ have led to the development of an early afterglow interpretation of high-energy radiation from the external forward shock (Kumar \& Barniol Duran 2009; Ackermann et al. 2010a; Corsi et al. 2010; Ghisellini et al. 2010; Razzaque 2010). The PL spectrum and temporally extended emission are then interpreted as synchrotron radiation from the external shock. Modeling of simultaneous multiwavelength (optical to $\mathrm{GeV}$ ) data has shown this approach to generally fit observations for several GRBs, such as GRB 090510, GRB 110731A, and GRB 130427A. However, detection of $\gtrsim 10 \mathrm{GeV}$ photons at late times from a number of GRBs (see Figure 25) is not easy to explain with the synchrotron interpretation (nor are the VHE photons from GRB $190114 \mathrm{C}$ seen by MAGIC). The maximum photon energy from electron-synchrotron radiation is $h \nu_{\max } \approx 0.24 \phi^{-1}(1+z)^{-1} \Gamma$ ( $t) \mathrm{GeV}$, where $\phi^{-1} \lesssim 1$ is the acceleration efficiency for electrons and $\Gamma(t)$ is the bulk Lorentz factor (see, e.g., Razzaque 2010).

In light of the difficulties in simultaneously matching both the delayed onset and long duration of $\mathrm{GeV}$ emission, alternative models have also been proposed. For instance, several authors have pointed out that the prompt $\mathrm{MeV}$ emission should cause the creation of $e^{ \pm}$pairs in the medium ahead of the blast wave (e.g., Thompson \& Madau 2000; Mészáros et al. 2001; Beloborodov 2002). This pair loading led Beloborodov (2005) to suggest that $\mathrm{GeV}$ emission arises owing to inverse Compton scattering of the prompt $\mathrm{MeV}$ radiation streaming through the external medium.

In Beloborodov et al. (2014), this scenario was shown to predict a delayed onset of $\sim 1-10 \mathrm{~s}$, in agreement with the majority of LAT GRBs. However, it is unclear whether also the much longer $(>100 \mathrm{~s})$ delays seen in a few GRBs can be explained. This would require either a very low Lorentz factor of the blast wave (not easy to reconcile with $\mathrm{GeV}$ emission) or a suppression of the blast-wave luminosity until it has reached a very large radius. Likewise, while the model can explain a $\mathrm{GeV}$ duration several times greater than that at lower energies, it may face difficulties for the LAT GRBs where the GeV duration is more than an order of magnitude longer (see panel (c) of Figure 7). Observational tests so far have also predicted rather large densities of the circumburst medium (comparable to stellar winds of $\sim 10^{-5} M_{\odot} \mathrm{yr}^{-1}$ ), which may prove challenging as more sGRBs are detected, as binary neutron stars are not expected to have a dense surrounding medium. 


\subsection{Fluence and Energetics of LAT GRBs: Probing the Central Engine}

LAT GRBs constitute a subsample of the GBM GRBs with a flux distribution peaking at a higher value than the complete GBM sample (see Figure 13). This is also true for the fluence as evident from the same figure. On the other hand, the redshift distribution of the LAT GRBs is rather typical of Swift-detected GRBs (see Figure 17). As a result, the isotropic energy release and peak luminosity (Figure 18) are also typically higher for the LAT GRBs across the redshifts. These results have implications for the GRB central engines.

The nature of the GRB central engine is a fundamental question in astrophysics. A newly born magnetar is one of the two leading hypotheses (the other is a newly born black hole). The vacuum/force-free electromagnetic spin-down power of a neutron star with surface (equatorial) dipole magnetic field $B_{\text {dip }}$ and spin period $P$ is given by (Spitkovsky 2006)

$$
\begin{aligned}
\dot{E}_{\mathrm{FF}} \approx & \frac{4 \pi^{4} B_{\mathrm{dip}}^{2} R^{6}}{P^{4} c^{3}} \sim 10^{49}\left(\frac{B_{\mathrm{dip}}}{10^{15} \mathrm{G}}\right)^{2} \\
& \times\left(\frac{P}{1 \mathrm{~ms}}\right)^{-4}\left(\frac{R}{10 \mathrm{~km}}\right)^{6} \mathrm{erg} \mathrm{s}^{-1},
\end{aligned}
$$

where $R$ is the neutron star radius. The maximum isotropic $\gamma$-ray power is then $\sim 10^{49}-10^{51} \mathrm{erg} \mathrm{s}^{-1}$, after taking into account a jet-beaming factor of up to $\sim 200$ for a $\sim 6^{\circ}$ jet opening angle. In comparison, the measured peak luminosity for a number of LAT-detected GRBs exceeds these values, e.g., GRB 080916C $\left(\approx 6 \times 10^{53} \mathrm{erg} \mathrm{s}^{-1}\right)$ and GRB 090510 and GRB 160625B $\left(\approx 4 \times 10^{53} \mathrm{erg} \mathrm{s}^{-1}\right)$. The observations of LAT GRBs therefore significantly constrain the parameter space of the models with a magnetar as the GRB central engine (see, e.g., Duncan \& Thompson 1992; Usov 1992; Metzger et al. 2011).

\subsection{Closure Relations: Test of the Standard External Forward Shock Model}

As described in Section 6.1, a possible explanation for the temporally extended emission is synchrotron emission from an external forward shock developed by collisions between outgoing ejecta and the circumburst medium. According to the model, a PL distribution of electrons radiates synchrotron emission, and the spectrum and corresponding light curve are described as a series of BPLs (e.g., Sari et al. 1998; Chevalier \& Li 2000; Zhang et al. 2006; Gao et al. 2013). The indices of each segment of both spectrum and corresponding light curve are either constant or a function of the electron spectral index, and the relation between the spectral and temporal indices is called the "closure relation." The closure relations have different forms depending on the physical conditions, such as the electron cooling regime, surrounding environment profile, electron spectral index, and jet geometry.

A systematic analysis of 59 GRBs testing a set of closure relations against temporal and spectral indices computed from LAT extended emission was performed in Tak et al. (2019, submitted). Among the analyzed GRBs, $81 \%$ of them can be classified by a set of closure relations. A third of these allow both interstellar medium (ISM) and wind environments, while the remainder favor an ISM environment. The observed spectra and light curves of the extended emission in LAT GRBs are thus generally consistent with the standard external forward shock model in most cases, but there is still a significant fraction that cannot be explained by this model. The GRBs that do not match any closure relation tend to show a slower temporal decay $(\alpha<1)$. This implies the need for continuous energy injection or other physical sources to sustain the fluxes.

As already mentioned in Section 5.6, some light curves show a break or even hints of a plateau at late times. Such features can cause problems for the models used above, and instead a more phenomenological approach needs to be adopted. Using the parameters derived from fitting the light curves with the Willingale et al. (2007) model, we tested also these GRBs against the closure relations. Unfortunately, due to the large uncertainties, no conclusive results could be reached at least at this stage. Thus, further analysis and future observations are required to cast light on this topic. Additional tests in other theoretical frameworks (e.g., the models proposed by Beloborodov et al. 2014; Ito et al. 2014) will be the object of a forthcoming paper.

\section{Summary and Conclusions}

We have here presented the second Fermi-LAT GRB catalog. Compared to the 1FLGC, there are several changes and improvements to the analysis, beyond the three times longer timescale:

1. Pass 8 data have replaced Pass 6 data.

2. Over the past $2 \mathrm{yr}$, a new detection algorithm has been developed at energies above $100 \mathrm{MeV}$.

3. A BB algorithm has been employed to look for detections at $30-100 \mathrm{MeV}$.

4. All available triggers (both GBM and other space-based missions) were used in the search for LAT counterparts.

All potential detections have been processed by a dedicated and standardized analysis. This differs from both the analysis performed for the 1FLGC and the standard LAT real-time analysis. In total we find 186 detections, a much larger sample than the 35 GRBs of the 1FLGC.

The results presented in the 2FLGC have strengthened some of the conclusions from the 1FLGC, most notably the temporal characteristics. A major difference from the 1FLGC is a new definition of the duration, where we consider the first and last detected photon. Nevertheless, the properties of later onset and longer duration for the high-energy emission remain unchanged. Other trends suggested by the 1FLGC, most notably regarding the energetics and differences between long and short GRBs, have been shown not to hold with the larger sample in the 2FLGC.

While this catalog describes the temporal properties of the detections at both $\mathrm{keV}$ and $\mathrm{MeV}$ energy ranges, the spectral analysis is limited to the LAT range $(100 \mathrm{MeV}-100 \mathrm{GeV})$. This has shown to be problematic in some cases, such as when testing for breaks in the spectrum. These results are not surprising, as the division between the energy ranges is purely artifical. Nevertheless, a joint analysis is beyond the scope of this paper and will instead be the focus of a dedicated study presented in a separate publication.

The larger sample in this catalog is also reflected in the 34 GRBs with measured redshift. This has allowed us to better investigate the energetics also in the rest frame, probing both the highest-energy photons and the closure relations. The remarkable alignment seen in the decay of the luminosity further points to a strong correlation between isotropic 
luminosity in the rest-frame $\mathrm{MeV}-\mathrm{GeV}$ range and isotropic energy as measured at lower energies.

The 2FLGC presents the results of the first $10 \mathrm{yr}$ of FermiLAT; it represents an initial step in understanding the processes behind high-energy emission from gamma-ray bursts. It is our hope that the Fermi data presented here will lead to a large number of follow-up analyses and continue to drive the evolution of theoretical models. Within the Fermi-LAT Collaboration, several such studies are already underway: focused studies on the closure relations, energetics, and the connection to VHE emission. The large sample of the 2FLGC will also allow more complete analysis of the detectability of $\mathrm{MeV}$ emission from GRBs, as well as detailed comparison between the energy output in the LAT range during the prompt versus afterglow phases. The collaboration is also in the process of setting up an online repository, similar to the analysis of the 2FLGC, which will automatically be updated with each new GRB detection. The past few years have truly shown the strength of Fermi observations, and its importance will continue for many years to come. This is especially true as the era of multimessenger studies of GRBs has just begun.

The Fermi-LAT Collaboration acknowledges generous ongoing support from a number of agencies and institutes that have supported both the development and the operation of the LAT, as well as scientific data analysis. These include the National Aeronautics and Space Administration and the Department of Energy in the United States; the Commissariat à l'Energie Atomique and the Centre National de la Recherche Scientifique/Institut National de Physique Nucléaire et de Physique des Particules in France; the Agenzia Spaziale Italiana and the Istituto Nazionale di Fisica Nucleare in Italy; the Ministry of Education, Culture, Sports, Science and Technology (MEXT), High Energy Accelerator Research Organization (KEK), and Japan Aerospace Exploration Agency (JAXA) in Japan; and the K. A. Wallenberg Foundation, the Swedish Research Council, and the Swedish National Space Agency in Sweden.

Additional support for science analysis during the operations phase is gratefully acknowledged from the Istituto Nazionale di Astrofisica in Italy and the Centre National d'Études Spatiales in France. This work performed in part under DOE Contract DE-AC02-76SF00515. M. Axelsson gratefully acknowledges funding from the European Union's Horizon 2020 research and innovation program under the Marie Skłodowska-Curie grant agreement No. 734303 (NEWS).

\section{Appendix A Tables}

\section{A1. Sample of LAT Bursts}

The complete list of the Fermi-LAT $10 \mathrm{yr}$ catalog GRBs is presented in Table 2. The first column gives the GRB name as it appeared in the corresponding GCN. The following two columns display the GRB trigger date (in UTC) and time (in Mission Elapsed Time, or MET, in seconds from 2001 January 1 UT 00:00:00). Columns (4) through (6) report the best reconstructed position using only Fermi-LAT data. Coordinates are expressed in the equatorial frame of epoch J2000. The localization error (Column (6)) is the error obtained from the LTF analysis. The boresight angle $\theta$ and the zenith angle $\zeta$ at trigger time are given in Columns (7) and (8). Column (9) indicates the presence or absence of an ARR. The detection significance of the LTF and LLE analysis are listed in Columns (10) and (11). The LTF TS value corresponds to the highest TS obtained during one of the four time windows listed in Table 1. LLE-only events, for which no position calculation was possible, report the best location available for that event. The reference to the burst location is then given in Column (12). When available, we report the redshift of the host galaxy in Column (13), together with its corresponding reference (Column (14)).

\section{A2. Comparison of Duration Estimation}

Table 3 presents the different burst duration estimations. The GRB class (listed in Column (2); $\mathrm{L}=$ long, $\mathrm{S}=$ short) is derived from the GBM duration in the $50-300 \mathrm{keV}$ energy range (long bursts have $T_{\mathrm{GBM}, 90}>2 \mathrm{~s}$, and short bursts have $\left.T_{\mathrm{GBM}, 90}<2 \mathrm{~s}\right)$. All GBM values $\left(T_{\mathrm{GBM}, 05}, T_{\mathrm{GBM}, 95}\right.$, and

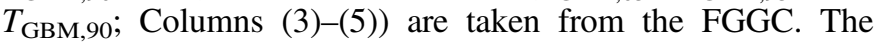
LLE onset times and durations $\left(T_{\text {LLE,05 }}, T_{\text {LLE,95, and }} T_{\text {LLE,90 }}\right)$, listed in Columns (6)-(8), are calculated in the $30 \mathrm{MeV}-1 \mathrm{GeV}$ energy range. The LAT onset time $T_{\mathrm{LAT}, 0}$, end time $T_{\mathrm{LAT}, 1}$, and duration $T_{\mathrm{LAT}, 100}$, calculated in the $100 \mathrm{MeV}-100 \mathrm{GeV}$ energy range, are given in Columns (9)-(11).

\section{A3. Likelihood Analysis in Different Time Window}

Table 4 reports the likelihood analysis results in the different time windows. The definition of the time windows is explained in Table 1. The time range of each interval in which we perform the analysis is given in Column (2). Columns (3) and (4) display the number of selected events in the ROI and the number of events predicted by the best-fit model obtained after likelihood maximization, respectively. The TS is given in Column (5). Note that only intervals with $\mathrm{TS}>20$ are displayed in the table. Columns (6) through (9) contain the value of the spectral index, flux, and fluence in each interval and, when possible, the isotropic energy calculated in the $100 \mathrm{MeV}-100 \mathrm{GeV}$ rest-frame energy band.

\section{A4. Temporally Extended High-energy Emission}

Table 5 displays the temporally extended high-energy emission analysis results. Columns (2) and (3) give the peak flux and peak flux time. These are followed by the fit parameters of the simple PL ( $\alpha$; Column (4)) and BPL $\left(\alpha_{1}, \alpha_{2}\right.$, and $T_{b}$; Columns (5)-(7)). Column (8) reports the best-fit decay index, indicating whether the SPL or the BPL is the preferred model. The last column indicates the total duration of the extended emission.

\section{A5. Highest-energy Events}

For each GRB, Table 6 shows the highest-energy photon detected in the GBM time window (Columns (2)-(5)), as well as the highest-energy photon found overall in the time-resolved analysis (Columns (6)-(9)). For each time span the values given are the total number of photons found with probability $>90 \%$ to be associated with the GRB, the energy of the most energetic photon, its arrival time, and the probability to be associated with the GRB.

Table 7 lists the 29 GRBs from which at least one photon with energy greater than $10 \mathrm{GeV}$ has been detected. For each such photon, the energy and arrival time are given. For GRBs with a measured redshift, this is listed along with the corresponding source-frame energy $\left(E_{\mathrm{sf}}\right)$ of each photon. 
Table 2

Sample of Fermi-LAT GRBs, from 2008 to 2018 August

\begin{tabular}{|c|c|c|c|c|c|c|c|c|c|c|c|c|c|}
\hline GRB Name & $\begin{array}{l}\text { Date } \\
\text { (UT) }\end{array}$ & $\begin{array}{l}\text { Trigger Time } \\
\text { (MET) }\end{array}$ & $\begin{array}{l}\text { R.A. } \\
\text { (deg, } \\
\text { J2000) }\end{array}$ & $\begin{array}{c}\text { Decl. } \\
(\mathrm{deg}, \mathrm{J} 2000)\end{array}$ & $\begin{array}{l}\text { Loc. Err. } \\
\text { (deg) }\end{array}$ & $\begin{array}{c}\theta \\
(\mathrm{deg})\end{array}$ & $\begin{array}{c}\zeta \\
(\mathrm{deg})\end{array}$ & ARR & LTF TS & $\begin{array}{l}\text { LLE Sig. } \\
\qquad(\sigma)\end{array}$ & Loc. GCN & Redshift & GCN \\
\hline 080818B & 2008 Aug 18 22:40:49 & 240792050.080 & 109.46 & 15.0 & 0.47 & 56.4 & 75.0 & 0 & 26 & $\ldots$ & & $\ldots$ & \\
\hline $080825 \mathrm{C}$ & 2008 Aug 25 14:13:48 & 241366429.110 & 234.83 & -3.84 & 0.65 & 60.2 & 26.3 & 0 & 56 & 8 & & $\ldots$ & \\
\hline $080916 \mathrm{C}$ & 2008 Sep 16 00:12:45 & 243216766.620 & 119.89 & -56.7 & 0.07 & 49.0 & 83.8 & 0 & 2200 & 46 & & 4.35 & Greiner et al. (2009) \\
\hline 081006 & 2008 Oct 6 14:29:34 & 244996175.170 & 135.0 & -61.95 & 0.38 & 10.8 & 40.3 & 0 & 97 & $\ldots$ & & $\ldots$ & \\
\hline 081009 & 2008 Oct 9 03:20:58 & 245215259.060 & 251.57 & 18.92 & 0.28 & 69.6 & 55.4 & 0 & 50 & $\ldots$ & & $\ldots$ & \\
\hline 081024B & 2008 Oct 24 21:22:40 & 246576161.860 & 323.01 & 20.84 & 0.31 & 19.2 & 30.6 & 0 & 83 & 8 & & $\ldots$ & \\
\hline 081102B & 2008 Nov 2 08:45:00 & 247308301.510 & 212.95 & 30.33 & 0.58 & 48.2 & 47.6 & 0 & 26 & $\ldots$ & & $\ldots$ & \\
\hline 081122A & 2008 Nov 22 12:28:12 & 249049693.210 & 338.82 & 37.57 & 0.27 & 21.5 & 25.9 & 1 & 38 & $\ldots$ & & $\ldots$ & \\
\hline 081203A & 2008 Dec 3 13:57:11 & 250005432.570 & 233.49 & 63.96 & 0.39 & 78.0 & 106.1 & 0 & 30 & $\ldots$ & & 2.10 & Landsman et al. (2008) \\
\hline 081224 & 2008 Dec 24 21:17:55 & 251846276.410 & 201.1 & 72.67 & 0.33 & 15.4 & 49.2 & 1 & 29 & $\ldots$ & & $\ldots$ & \\
\hline 090102 & 2009 Jan 2 02:55:30 & 252557732.850 & 127.56 & 33.46 & 0.65 & 85.5 & 78.7 & 0 & 20 & $\cdots$ & & 1.55 & $\begin{array}{l}\text { de Ugarte Postigo et al. } \\
\text { (2009) }\end{array}$ \\
\hline 090217 & 2009 Feb 17 04:56:42 & 256539404.560 & 205.01 & -8.39 & 0.46 & 33.7 & 46.2 & 0 & 100 & 16 & & $\cdots$ & \\
\hline 090227A & 2009 Feb 27 07:25:57 & 257412359.000 & 4.56 & -44.63 & 0.34 & 21.6 & 45.4 & 0 & 33 & 6 & & $\cdots$ & \\
\hline 090227B & 2009 Feb 27 18:31:01 & 257452263.407 & 11.8 & 32.2 & 1.80 & 72.7 & 86.6 & 1 & $\ldots$ & 15 & FGGC & $\ldots$ & \\
\hline 090228A & 2009 Feb 28 04:53:20 & 257489602.920 & 98.6 & -28.79 & 0.21 & 23.8 & 56.6 & 1 & 41 & $\ldots$ & & $\ldots$ & \\
\hline 090323 & 2009 Mar 23 00:02:42 & 259459364.630 & 190.75 & 17.07 & 0.09 & 56.5 & 66.2 & 1 & 230 & 18 & & 3.57 & Chornock et al. (2009) \\
\hline 090328 & 2009 Mar 28 09:36:46 & 259925808.510 & 90.67 & -41.95 & 0.12 & 65.7 & 72.4 & 1 & 290 & 20 & & 0.74 & Cenko et al. (2009) \\
\hline 090427A & 2009 Apr 27 23:26:32 & 262567594.000 & 235.86 & -14.05 & 0.11 & 134.0 & 135.4 & 0 & 40 & $\cdots$ & & $\ldots$ & \\
\hline 090510 & 2009 May 10 00:22:59 & 263607781.970 & 333.57 & -26.62 & 0.05 & 13.5 & 52.3 & 1 & 2600 & 24 & & 0.90 & Rau et al. (2009) \\
\hline 090531B & 2009 May 31 18:35:56 & 265487758.492 & 252.06 & -36.05 & $\cdots$ & 21.9 & 49.2 & 0 & $\cdots$ & 10 & FGGC & $\cdots$ & \\
\hline 090626 & 2009 Jun 26 04:32:08 & 267683530.880 & 170.19 & -33.56 & 0.29 & 22.6 & 10.4 & 0 & 100 & 9 & & $\cdots$ & \\
\hline 090720B & 2009 Jul 20 17:02:56 & 269802178.900 & 203.638 & -51.198 & 0.99 & 56.3 & 94.2 & 1 & 35 & $\ldots$ & & $\ldots$ & \\
\hline 090902B & 2009 Sep 2 11:05:08 & 273582310.320 & 264.95 & 27.33 & 0.04 & 50.3 & 37.6 & 1 & 3600 & 8 & & 1.82 & Cucchiara et al. (2009c) \\
\hline 090926A & 2009 Sep 26 04:20:26 & 275631628.990 & 353.56 & -66.37 & 0.06 & 48.0 & 80.4 & 1 & 3200 & 29 & & 2.11 & Malesani et al. (2009) \\
\hline 091003 & 2009 Oct 3 04:35:45 & 276237347.580 & 251.54 & 36.62 & 0.10 & 12.2 & 62.1 & 1 & 170 & $\ldots$ & & 0.90 & Cucchiara et al. (2009b) \\
\hline 091031 & 2009 Oct 31 12:00:28 & 278683230.850 & 71.81 & -57.73 & 0.23 & 24.6 & 39.4 & 0 & 110 & 17 & & $\ldots$ & \\
\hline 091120 & 2009 Nov 20 04:34:40 & 280384482.230 & 226.7 & -21.36 & 0.18 & 48.1 & 25.7 & 1 & 38 & $\ldots$ & & $\ldots$ & \\
\hline 091127 & 2009 Nov 27 23:25:45 & 281057147.490 & 36.65 & -19.14 & 0.31 & 25.8 & 54.5 & 1 & 34 & $\ldots$ & & 0.49 & Cucchiara et al. (2009a) \\
\hline 100116A & 2010 Jan 16 21:31:00 & 285370262.240 & 305.0 & 14.51 & 0.06 & 25.9 & 36.3 & 0 & 130 & 31 & & $\ldots$ & \\
\hline $100213 \mathrm{C}$ & 2010 Feb 13 12:44:41 & 287757883.000 & 282.45 & 15.15 & 0.06 & 146.4 & 147.2 & 0 & 45 & $\ldots$ & & $\ldots$ & \\
\hline $100225 \mathrm{~A}$ & 2010 Feb 25 02:45:31 & 288758733.150 & 311.93 & -60.51 & 0.34 & 57.0 & 83.7 & 0 & 47 & 10 & & $\cdots$ & \\
\hline $100325 \mathrm{~A}$ & 2010 Mar 25 06:36:08 & 291191770.030 & 330.26 & -26.99 & 0.87 & 7.8 & 52.9 & 0 & 30 & $\cdots$ & & $\cdots$ & \\
\hline $100414 \mathrm{~A}$ & 2010 Apr 14 02:20:21 & 292904423.990 & 192.15 & 8.56 & 0.09 & 69.6 & 29.0 & 1 & 190 & $\cdots$ & & 1.37 & Cucchiara \& Fox (2010) \\
\hline $100423 B$ & 2010 Apr 23 05:51:25 & 293694687.750 & 120.18 & 13.36 & 0.19 & 47.9 & 32.8 & 0 & 31 & $\cdots$ & & $\cdots$ & \\
\hline $100511 \mathrm{~A}$ & 2010 May 11 00:49:56 & 295231798.230 & 111.38 & -0.99 & 0.07 & 48.0 & 26.2 & 1 & 93 & $\cdots$ & & $\cdots$ & \\
\hline $100620 \mathrm{~A}$ & 2010 Jun 20 02:51:29 & 298695091.120 & 87.68 & -50.66 & 1.24 & 25.2 & 73.3 & 0 & 29 & $\cdots$ & & $\cdots$ & \\
\hline 100724B & 2010 Jul 24 00:42:06 & 301624928.090 & 123.59 & 75.86 & 0.10 & 50.0 & 96.2 & 1 & 110 & 40 & & $\cdots$ & \\
\hline $100728 \mathrm{~A}$ & $2010 \mathrm{Jul} 28$ 02:17:30 & 301976252.610 & 88.79 & -15.41 & 0.18 & 60.5 & 36.9 & 1 & 63 & $\cdots$ & & 1.57 & Kruehler et al. (2010) \\
\hline $100826 \mathrm{~A}$ & 2010 Aug 26 22:58:22 & 304556304.900 & 277.71 & -25.27 & 1.13 & 73.0 & 85.1 & 1 & 29 & 21 & & $\cdots$ & \\
\hline $101014 \mathrm{~A}$ & 2010 Oct 14 04:11:52 & 308722314.620 & 26.5 & -48.54 & 0.09 & 55.0 & 102.6 & 1 & 67 & 12 & & $\cdots$ & \\
\hline $101107 \mathrm{~A}$ & 2010 Nov 7 00:16:25 & 310781787.120 & 170.27 & 23.88 & 0.20 & 36.6 & 28.4 & 0 & 47 & $\cdots$ & & $\cdots$ & \\
\hline $101123 \mathrm{~A}$ & 2010 Nov 23 22:51:34 & 312245496.973 & 135.16 & 1.91 & 1.00 & 77.4 & 97.8 & 0 & $\cdots$ & 8 & FGGC & $\cdots$ & \\
\hline $101227 \mathrm{~B}$ & 2010 Dec 27 09:45:06 & 315135908.570 & 240.21 & -15.93 & 0.32 & 13.7 & 37.3 & 0 & 27 & $\cdots$ & & $\cdots$ & \\
\hline $110120 \mathrm{~A}$ & 2011 Jan 20 15:59:39 & 317231981.230 & 61.62 & -12.02 & 0.20 & 14.6 & 31.5 & 1 & 67 & 6 & & $\cdots$ & \\
\hline $110123 \mathrm{~A}$ & 2011 Jan 23 19:17:45 & 317503067.050 & 240.61 & 28.37 & 0.22 & 55.4 & 91.9 & 1 & 62 & $\cdots$ & & $\cdots$ & \\
\hline
\end{tabular}


Table 2

(Continued)

\begin{tabular}{|c|c|c|c|c|c|c|c|c|c|c|c|c|c|}
\hline GRB Name & $\begin{array}{l}\text { Date } \\
\text { (UT) }\end{array}$ & $\begin{array}{c}\text { Trigger Time } \\
\text { (MET) }\end{array}$ & $\begin{array}{l}\text { R.A. } \\
\text { (deg, } \\
\text { J2000) }\end{array}$ & $\begin{array}{c}\text { Decl. } \\
\text { (deg, J2000) }\end{array}$ & $\begin{array}{l}\text { Loc. Err. } \\
\qquad(\mathrm{deg})\end{array}$ & $\begin{array}{c}\theta \\
(\mathrm{deg})\end{array}$ & $\begin{array}{c}\zeta \\
(\mathrm{deg})\end{array}$ & ARR & LTF TS & $\begin{array}{l}\text { LLE Sig. } \\
\qquad(\sigma)\end{array}$ & Loc. GCN & Redshift & GCN \\
\hline $110213 \mathrm{~A}$ & 2011 Feb 13 05:17:11 & 319267033.280 & 42.98 & 49.11 & 0.22 & 98.6 & 53.2 & 0 & 28 & $\ldots$ & & 1.46 & Milne \& Cenko (2011) \\
\hline 110328B & 2011 Mar 28 12:29:19 & 323008161.190 & 121.66 & 41.52 & 0.16 & 36.0 & 43.3 & 0 & 50 & 29 & & $\ldots$ & \\
\hline $110428 \mathrm{~A}$ & 2011 Apr 28 09:18:30 & 325675112.400 & 5.42 & 65.01 & 0.18 & 34.6 & 84.3 & 0 & 52 & $\cdots$ & & $\cdots$ & \\
\hline $110518 \mathrm{~A}$ & 2011 May 18 20:38:10 & 327443892.000 & 67.19 & -34.27 & 0.19 & 165.9 & 124.1 & 0 & 40 & $\cdots$ & & $\cdots$ & \\
\hline $110529 \mathrm{~A}$ & 2011 May 29 00:48:42 & 328322924.870 & 118.33 & 67.91 & 1.50 & 30.0 & 79.9 & 1 & $\cdots$ & 15 & FGGC & $\cdots$ & \\
\hline $110625 \mathrm{~A}$ & 2011 Jun 25 21:08:18 & 330728900.230 & 286.38 & 6.4 & 0.13 & 87.9 & 51.4 & 1 & 55 & $\ldots$ & & $\cdots$ & \\
\hline $110721 \mathrm{~A}$ & 2011 Jul 21 04:47:43 & 332916465.760 & 333.53 & -38.58 & 0.12 & 40.2 & 82.9 & 1 & 220 & 61 & & $\cdots$ & \\
\hline $110728 \mathrm{~A}$ & $2011 \mathrm{Jul} 28$ 01:20:22 & 333508824.810 & 173.57 & 4.34 & 0.62 & 41.7 & 16.8 & 0 & 43 & $\ldots$ & & $\ldots$ & \\
\hline $110731 \mathrm{~A}$ & 2011 Jul 31 11:09:29 & 333803371.950 & 280.42 & -28.65 & 0.24 & 2.9 & 50.7 & 1 & 390 & 20 & & 2.83 & Tanvir et al. (2011) \\
\hline $110903 \mathrm{~A}$ & 2011 Sep 3 02:39:34 & 336710376.420 & 197.26 & 58.85 & 0.07 & 44.3 & 93.9 & 0 & 49 & $\cdots$ & & $\cdots$ & \\
\hline 110921B & 2011 Sep 21 21:52:45 & 338334767.090 & 5.77 & -26.59 & 0.39 & 33.9 & 21.2 & 1 & 50 & 7 & & $\cdots$ & \\
\hline 111210B & 2011 Dec 10 08:37:39 & 345199061.000 & 122.51 & 44.09 & 0.82 & 28.3 & 24.1 & 0 & 34 & $\cdots$ & & $\cdots$ & \\
\hline 120107A & 2012 Jan 7 09:12:15 & 347620337.410 & 245.01 & -70.59 & 0.34 & 46.8 & 96.7 & 0 & 30 & $\cdots$ & & $\cdots$ & \\
\hline $120226 \mathrm{~A}$ & 2012 Feb 26 20:54:17 & 351982459.030 & 302.97 & 48.98 & 0.68 & 37.1 & 42.2 & 1 & 37 & 12 & & $\cdots$ & \\
\hline $120316 \mathrm{~A}$ & 2012 Mar 16 00:11:02 & 353549464.560 & 57.35 & -56.41 & 0.31 & 9.9 & 48.7 & 0 & 57 & 5 & & $\cdots$ & \\
\hline 120328B & 2012 Mar 28 06:26:20 & 354608782.953 & 228.14 & 22.8 & 1.00 & 68.5 & 106.6 & 0 & $\ldots$ & 8 & FGGC & $\ldots$ & \\
\hline 120420B & 2012 Apr 20 20:35:13 & 356646915.070 & 108.77 & 15.17 & 0.29 & 109.7 & 76.8 & 0 & 29 & $\ldots$ & & $\ldots$ & \\
\hline $120526 \mathrm{~A}$ & 2012 May 26 07:16:40 & 359709402.760 & 53.82 & -35.23 & 0.11 & 109.7 & 62.9 & 0 & 110 & $\cdots$ & & $\ldots$ & \\
\hline 120624B & 2012 Jun 24 22:23:54 & 362269436.930 & 170.91 & 8.94 & 0.21 & 68.2 & 26.6 & 1 & 450 & 9 & & 2.20 & $\begin{array}{l}\text { de Ugarte Postigo et al. } \\
\text { (2013a) }\end{array}$ \\
\hline 120709A & 2012 Jul 9 21:11:40 & 363561103.370 & 318.8 & -50.42 & 0.40 & 27.8 & 40.5 & 0 & 84 & 9 & & $\ldots$ & \\
\hline $120711 \mathrm{~A}$ & 2012 Jul 11 02:44:53 & 363667496.290 & 94.64 & -70.88 & 0.13 & 134.5 & 85.0 & 1 & 140 & $\cdots$ & & 1.41 & Tanvir et al. (2012) \\
\hline 120729A & 2012 Jul 29 10:56:12 & 365252175.660 & 13.26 & 49.91 & 0.38 & 82.6 & 32.7 & 0 & 35 & $\cdots$ & & 0.80 & Tanvir \& Ball (2012) \\
\hline $120830 \mathrm{~A}$ & 2012 Aug 30 07:07:03 & 368003226.540 & 88.59 & -28.79 & 0.62 & 38.7 & 75.3 & 0 & 44 & $\cdots$ & & $\cdots$ & \\
\hline 120911B & 2012 Sep 11 06:26:12 & 369037575.000 & 172.66 & -37.5 & 0.60 & 66.1 & 22.2 & 0 & 340 & 18 & & $\cdots$ & \\
\hline $120915 \mathrm{~A}$ & 2012 Sep 15 00:00:41 & 369360044.640 & 240.95 & 57.04 & 1.76 & 10.8 & 59.2 & 0 & 32 & $\ldots$ & & $\cdots$ & \\
\hline 120919B & 2012 Sep 19 01:14:23 & 369710066.070 & 302.73 & -37.73 & 0.14 & 22.9 & 65.9 & 0 & 30 & $\ldots$ & & $\ldots$ & \\
\hline $121011 \mathrm{~A}$ & 2012 Oct 11 11:15:25 & 371646928.697 & 260.2 & 41.14 & 0.01 & 55.9 & 84.8 & 0 & $\ldots$ & 8 & GCN13845 & $\cdots$ & \\
\hline $121029 \mathrm{~A}$ & 2012 Oct 29 08:24:19 & 373191862.930 & 229.07 & -25.56 & 0.21 & 61.2 & 42.0 & 1 & 40 & $\ldots$ & & $\cdots$ & \\
\hline 121123B & 2012 Nov 23 10:35:55 & 375359758.710 & 28.62 & -24.06 & 0.50 & 78.8 & 76.4 & 0 & 27 & $\ldots$ & & $\ldots$ & \\
\hline 121225B & 2012 Dec 25 10:00:53 & 378122456.583 & 310.45 & -34.83 & 1.50 & 64.3 & 90.7 & 0 & $\ldots$ & 8 & FGGC & $\ldots$ & \\
\hline $130305 \mathrm{~A}$ & 2013 Mar 5 11:39:11 & 384176354.369 & 116.765 & 52.025 & 0.06 & 41.5 & 99.6 & 0 & $\ldots$ & 19 & GCN14257 & $\ldots$ & \\
\hline $130310 \mathrm{~A}$ & 2013 Mar 10 20:09:41 & 384638984.500 & 142.38 & -17.1 & 0.47 & 75.6 & 49.8 & 1 & 47 & 11 & & $\ldots$ & \\
\hline $130325 \mathrm{~A}$ & 2013 Mar 25 04:51:54 & 385879917.300 & 122.97 & -19.15 & 0.18 & 47.3 & 76.1 & 1 & 60 & $\cdots$ & & $\cdots$ & \\
\hline 130327B & 2013 Mar 27 08:24:04 & 386065447.050 & 218.26 & -69.76 & 0.21 & 46.3 & 86.2 & 1 & 250 & $\cdots$ & & $\cdots$ & \\
\hline $130427 \mathrm{~A}$ & 2013 Apr 27 07:47:06 & 388741629.420 & 173.14 & 27.73 & 0.04 & 46.4 & 62.8 & 1 & 4200 & 15 & & 0.34 & Xu et al. (2013) \\
\hline 130502B & 2013 May 2 07:51:11 & 389173874.760 & 66.65 & 70.83 & 0.32 & 46.4 & 96.4 & 0 & 330 & 8 & & $\cdots$ & \\
\hline $130504 \mathrm{C}$ & 2013 May 4 23:28:57 & 389402940.520 & 91.55 & 3.78 & 0.12 & 47.6 & 52.3 & 1 & 91 & 29 & & $\cdots$ & \\
\hline $130518 \mathrm{~A}$ & 2013 May 18 13:54:37 & 390578080.530 & 355.59 & 47.53 & 0.19 & 40.3 & 82.4 & 1 & 140 & 19 & & 2.49 & $\begin{array}{l}\text { Sanchez-Ramirez et al. } \\
\text { (2013) }\end{array}$ \\
\hline 130606B & 2013 Jun 6 11:55:33 & 392212536.630 & 218.57 & -22.15 & 0.11 & 90.5 & 10.9 & 1 & 87 & $\cdots$ & & $\cdots$ & \\
\hline $130702 \mathrm{~A}$ & 2013 Jul 2 00:05:23 & 394416326.080 & 216.39 & 16.15 & 0.62 & 76.7 & 78.9 & 0 & 26 & $\ldots$ & & 0.14 & Leloudas et al. (2013) \\
\hline $130821 \mathrm{~A}$ & 2013 Aug 21 16:10:28 & 398794231.010 & 314.23 & -11.52 & 0.18 & 34.9 & 49.2 & 1 & 170 & 16 & & $\cdots$ & \\
\hline $130828 \mathrm{~A}$ & 2013 Aug 28 07:20:00 & 399367203.150 & 259.72 & 28.21 & 0.29 & 39.9 & 45.5 & 1 & 150 & 9 & & $\ldots$ & \\
\hline 130907A & 2013 Sep 7 21:41:13 & 400282876.090 & 215.92 & 45.7 & 0.26 & 105.4 & 57.2 & 0 & 30 & $\cdots$ & & 1.24 & $\begin{array}{l}\text { de Ugarte Postigo et al. } \\
\text { (2013c) }\end{array}$ \\
\hline
\end{tabular}


Table 2

(Continued)

\begin{tabular}{|c|c|c|c|c|c|c|c|c|c|c|c|c|c|}
\hline GRB Name & $\begin{array}{l}\text { Date } \\
\text { (UT) }\end{array}$ & $\begin{array}{l}\text { Trigger Time } \\
\text { (MET) }\end{array}$ & $\begin{array}{l}\text { R.A. } \\
\text { (deg, } \\
\text { J2000) }\end{array}$ & $\begin{array}{c}\text { Decl. } \\
(\mathrm{deg}, \mathrm{J} 2000)\end{array}$ & $\begin{array}{l}\text { Loc. Err. } \\
\text { (deg) }\end{array}$ & $\begin{array}{c}\theta \\
(\operatorname{deg})\end{array}$ & $\begin{array}{c}\zeta \\
(\operatorname{deg})\end{array}$ & ARR & LTF TS & $\begin{array}{l}\text { LLE Sig. } \\
\qquad(\sigma)\end{array}$ & Loc. GCN & Redshift & GCN \\
\hline $131014 \mathrm{~A}$ & 2013 Oct 14 05:09:00 & 403420143.200 & 100.78 & -20.76 & 0.81 & 72.5 & 76.0 & 1 & 98 & 17 & & $\cdots$ & \\
\hline 131029A & 2013 Oct 29 23:20:48 & 404781651.580 & 200.85 & 48.03 & 0.18 & 59.8 & 94.1 & 0 & 110 & $\cdots$ & & $\ldots$ & \\
\hline $131108 \mathrm{~A}$ & 2013 Nov 8 20:41:55 & 405636118.760 & 156.81 & 9.57 & 0.22 & 20.4 & 21.9 & 1 & 1200 & 37 & & 2.40 & $\begin{array}{l}\text { de Ugarte Postigo et al. } \\
\text { (2013b) }\end{array}$ \\
\hline 131209A & 2013 Dec 9 13:07:56 & 408287279.960 & 137.06 & -33.9 & 1.16 & 19.8 & 53.6 & 0 & 29 & $\ldots$ & & $\cdots$ & \\
\hline $131216 \mathrm{~A}$ & 2013 Dec 16 01:56:32 & 408851795.063 & 91.59 & -35.5 & 2.24 & 44.6 & 77.2 & 1 & $\cdots$ & 7 & FGGC & $\cdots$ & \\
\hline $131231 \mathrm{~A}$ & 2013 Dec 31 04:45:16 & 410157919.080 & 10.55 & -1.72 & 0.05 & 37.2 & 39.9 & 1 & 240 & 18 & & 0.64 & Xu et al. (2014b) \\
\hline $140102 \mathrm{~A}$ & 2014 Jan 2 21:17:37 & 410390260.810 & 212.02 & 1.47 & 0.24 & 47.2 & 44.7 & 1 & 55 & 11 & & $\cdots$ & \\
\hline 140104B & 2014 Jan 4 17:32:03 & 410549526.150 & 218.76 & -8.98 & 0.18 & 17.6 & 36.5 & 0 & 62 & $\ldots$ & & $\ldots$ & \\
\hline 140110A & 2014 Jan 10 06:18:37 & 411027520.940 & 29.12 & -36.5 & 0.25 & 36.1 & 64.6 & 0 & 200 & 13 & & $\ldots$ & \\
\hline $140124 \mathrm{~A}$ & 2014 Jan 24 12:38:31 & 412259914.190 & 59.27 & 36.86 & 0.33 & 19.4 & 65.6 & 0 & 26 & $\ldots$ & & $\ldots$ & \\
\hline 140206B & 2014 Feb 6 06:36:12 & 413361375.840 & 315.24 & -8.79 & 0.08 & 46.2 & 86.0 & 1 & 300 & 39 & & $\ldots$ & \\
\hline 140219A & 2014 Feb 19 19:46:32 & 414531995.240 & 158.2 & 7.26 & 0.85 & 96.2 & 113.4 & 0 & 26 & $\cdots$ & & $\ldots$ & \\
\hline $140323 \mathrm{~A}$ & 2014 Mar 23 10:22:53 & 417262976.120 & 356.52 & -79.92 & 0.18 & 30.3 & 68.4 & 0 & 40 & $\ldots$ & & $\ldots$ & \\
\hline $140402 \mathrm{~A}$ & 2014 Apr 2 00:10:06 & 418090209.990 & 207.66 & 5.97 & 0.16 & 13.7 & 46.0 & 0 & 93 & 8 & & $\cdots$ & \\
\hline 140416A & 2014 Apr 16 01:26:40 & 419304403.930 & 35.74 & 42.1 & 0.10 & 138.8 & 94.8 & 0 & 34 & $\cdots$ & & $\cdots$ & \\
\hline $140523 \mathrm{~A}$ & 2014 May 23 03:05:57 & 422507160.630 & 133.09 & 24.83 & 0.38 & 55.4 & 26.4 & 0 & 180 & $\cdots$ & & $\cdots$ & \\
\hline $140528 \mathrm{~A}$ & 2014 May 28 20:05:22 & 423000325.750 & 280.59 & -59.46 & 0.36 & 46.2 & 66.3 & 1 & 31 & $\cdots$ & & $\cdots$ & \\
\hline 140619B & 2014 Jun 19 11:24:40 & 424869883.520 & 132.66 & -9.65 & 0.20 & 38.9 & 25.8 & 0 & 210 & 10 & & $\ldots$ & \\
\hline $140723 \mathrm{~A}$ & 2014 Jul 23 01:36:30 & 427772193.730 & 211.05 & -3.57 & 0.51 & 54.4 & 38.6 & 0 & 110 & 8 & & $\cdots$ & \\
\hline $140729 \mathrm{~A}$ & 2014 Jul 29 00:36:53 & 428287016.710 & 193.9 & 15.42 & 0.26 & 26.5 & 40.9 & 0 & 69 & $\cdots$ & & $\cdots$ & \\
\hline 140810A & 2014 Aug 10 18:46:10 & 429389173.090 & 118.93 & 27.4 & 0.07 & 123.5 & 89.3 & 1 & 79 & $\cdots$ & & $\ldots$ & \\
\hline $140825 \mathrm{~A}$ & 2014 Aug 25 06:55:25 & 430642528.000 & 89.01 & -12.16 & 0.57 & 113.4 & 112.0 & 0 & 19 & $\cdots$ & & $\cdots$ & \\
\hline $140928 \mathrm{~A}$ & 2014 Sep 28 10:29:53 & 433592996.550 & 43.76 & -55.92 & 0.10 & 110.0 & 111.3 & 0 & 57 & $\cdots$ & & $\cdots$ & \\
\hline 141012A & 2014 Oct 12 18:33:17 & 434831600.670 & 289.92 & -53.0 & 0.27 & 47.2 & 94.7 & 0 & 110 & $\ldots$ & & $\cdots$ & \\
\hline $141028 \mathrm{~A}$ & 2014 Oct 28 10:54:46 & 436186489.780 & 322.78 & -0.64 & 0.27 & 36.4 & 48.1 & 1 & 130 & 31 & & 2.33 & Xu et al. (2014a) \\
\hline $141102 \mathrm{~A}$ & 2014 Nov 2 12:51:39 & 436625502.260 & 209.07 & -46.73 & 0.68 & 52.3 & 53.5 & 1 & 34 & $\cdots$ & & $\cdots$ & \\
\hline 141113A & 2014 Nov 13 08:17:43 & 437559466.500 & 182.32 & 77.38 & 0.71 & 53.7 & 86.4 & 0 & 37 & $\cdots$ & & $\cdots$ & \\
\hline $141207 \mathrm{~A}$ & 2014 Dec 7 19:11:21 & 439672284.100 & 159.8 & 3.95 & 0.20 & 61.1 & 36.8 & 0 & 260 & 15 & & $\cdots$ & \\
\hline 141221B & 2014 Dec 21 21:31:48 & 440890311.780 & 99.12 & -66.9 & 0.33 & 38.7 & 84.3 & 0 & 31 & $\cdots$ & & $\cdots$ & \\
\hline $141222 \mathrm{~A}$ & 2014 Dec 22 07:08:57 & 440924940.390 & 178.0 & -57.31 & 0.08 & 45.9 & 93.5 & 0 & 150 & 8 & & $\ldots$ & \\
\hline 150118B & 2015 Jan 18 09:48:17 & 443267300.770 & 240.23 & -35.93 & 0.56 & 65.3 & 91.7 & 1 & 28 & 6 & & $\cdots$ & \\
\hline $150202 \mathrm{~B}$ & 2015 Feb 2 23:59:08 & 444614351.270 & 86.27 & 59.05 & 0.33 & 17.2 & 66.9 & 1 & 26 & 20 & & $\ldots$ & \\
\hline $150210 \mathrm{~A}$ & 2015 Feb 10 22:26:24 & 445299987.290 & 112.28 & 13.57 & 0.43 & 53.7 & 10.0 & 0 & 92 & 14 & & $\cdots$ & \\
\hline $150314 \mathrm{~A}$ & 2015 Mar 14 04:54:50 & 448001693.860 & 126.12 & 63.79 & 0.72 & 45.1 & 91.9 & 1 & 30 & 6 & & 1.76 & $\begin{array}{l}\text { de Ugarte Postigo et al. } \\
\text { (2015a) }\end{array}$ \\
\hline $150403 \mathrm{~A}$ & 2015 Apr 3 21:54:10 & 449790853.960 & 311.75 & -62.46 & 0.23 & 55.3 & 105.1 & 1 & 46 & 17 & & 2.06 & Pugliese et al. (2015) \\
\hline $150416 \mathrm{~A}$ & 2015 Apr 16 18:33:25 & 450902008.965 & 58.75 & 52.96 & 1.93 & 68.6 & 90.7 & 0 & $\cdots$ & 9 & FGGC & $\cdots$ & \\
\hline $150510 \mathrm{~A}$ & 2015 May 10 03:19:53 & 452920796.740 & 16.42 & 4.91 & 0.33 & 56.2 & 11.4 & 1 & 53 & 12 & & $\cdots$ & \\
\hline $150513 \mathrm{~A}$ & 2015 May 13 20:33:15 & 453241998.250 & 49.06 & -22.91 & 0.04 & 51.8 & 16.5 & 0 & $\cdots$ & 8 & GCN17810 & $\cdots$ & \\
\hline $150514 \mathrm{~A}$ & 2015 May 14 18:35:05 & 453321308.350 & 74.92 & -60.86 & 0.15 & 39.2 & 59.1 & 0 & 31 & $\cdots$ & & 0.81 & $\begin{array}{l}\text { de Ugarte Postigo et al. } \\
\text { (2015b) }\end{array}$ \\
\hline $150523 \mathrm{~A}$ & 2015 May 23 09:29:48 & 454066191.080 & 115.34 & -45.43 & 0.08 & 24.8 & 54.7 & 1 & 220 & 8 & & $\cdots$ & \\
\hline $150627 \mathrm{~A}$ & 2015 Jun 27 04:23:23 & 457071806.680 & 117.5 & -51.55 & 0.06 & 74.7 & 97.9 & 1 & 240 & 9 & & $\cdots$ & \\
\hline $150702 \mathrm{~A}$ & 2015 Jul 2 23:56:38 & 457574202.550 & 52.59 & -56.88 & 0.54 & 117.5 & 88.1 & 0 & 33 & $\cdots$ & & $\cdots$ & \\
\hline $150820 \mathrm{~A}$ & 2015 Aug 20 21:07:15 & 461797639.642 & 258.65 & -48.33 & 15.30 & 23.0 & 66.6 & 0 & $\cdots$ & 6 & FGGC & $\cdots$ & \\
\hline
\end{tabular}


Table 2

(Continued)

\begin{tabular}{|c|c|c|c|c|c|c|c|c|c|c|c|c|c|}
\hline GRB Name & $\begin{array}{l}\text { Date } \\
\text { (UT) }\end{array}$ & $\begin{array}{c}\text { Trigger Time } \\
\text { (MET) }\end{array}$ & $\begin{array}{l}\text { R.A. } \\
\text { (deg, } \\
\text { J2000) }\end{array}$ & $\begin{array}{c}\text { Decl. } \\
\text { (deg, J2000) }\end{array}$ & $\begin{array}{l}\text { Loc. Err. } \\
\qquad(\mathrm{deg})\end{array}$ & $\begin{array}{c}\theta \\
(\operatorname{deg})\end{array}$ & $\begin{array}{c}\zeta \\
(\mathrm{deg})\end{array}$ & ARR & LTF TS & $\begin{array}{l}\text { LLE Sig. } \\
\qquad(\sigma)\end{array}$ & Loc. GCN & Redshift & GCN \\
\hline $150902 \mathrm{~A}$ & 2015 Sep 2 17:35:39 & 462908143.360 & 214.95 & -69.38 & 0.18 & 37.3 & 87.1 & 1 & 140 & 21 & & $\ldots$ & \\
\hline 151006A & 2015 Oct 6 09:54:57 & 465818101.825 & 147.426 & 70.504 & 0.00 & 30.6 & 80.5 & 0 & $\ldots$ & 24 & GCN18403 & $\ldots$ & \\
\hline 160101B & 2016 Jan 1 05:10:12 & 473317816.860 & 1.36 & 55.23 & 1.37 & 9.0 & 59.0 & 0 & $\ldots$ & 13 & FGGC & $\ldots$ & \\
\hline $160310 \mathrm{~A}$ & 2016 Mar 10 00:22:58 & 479262182.470 & 98.78 & -7.12 & 0.06 & 62.8 & 29.9 & 0 & 28 & $\ldots$ & & $\ldots$ & \\
\hline 160314B & 2016 Mar 14 22:17:53 & 479686677.730 & 167.78 & 45.71 & 0.79 & 60.7 & 110.5 & 0 & 29 & $\ldots$ & & $\ldots$ & \\
\hline $160325 \mathrm{~A}$ & 2016 Mar 25 06:59:21 & 480581965.510 & 16.21 & -72.66 & 0.24 & 7.2 & 55.5 & 0 & 83 & 6 & & $\ldots$ & \\
\hline $160422 \mathrm{~A}$ & 2016 Apr 22 11:59:00 & 483019144.810 & 42.05 & -57.9 & 0.10 & 53.5 & 102.4 & 1 & 36 & $\ldots$ & & $\ldots$ & \\
\hline $160503 \mathrm{~A}$ & 2016 May 3 13:36:32 & 483975396.000 & 358.99 & -1.24 & 0.88 & 25.1 & 33.9 & 0 & 33 & $\ldots$ & & $\ldots$ & \\
\hline $160509 \mathrm{~A}$ & 2016 May 9 08:58:46 & 484477130.220 & 310.39 & 76.04 & 0.30 & 33.1 & 67.8 & 1 & 610 & 29 & & 1.17 & Tanvir et al. (2016) \\
\hline $160521 \mathrm{~B}$ & 2016 May 21 09:13:58 & 485514842.030 & 148.04 & 79.02 & 0.13 & 47.0 & 94.5 & 1 & 69 & $\ldots$ & & $\ldots$ & \\
\hline $160623 \mathrm{~A}$ & 2016 Jun 23 05:00:34 & 488350838.230 & 315.25 & 42.22 & 0.09 & 83.9 & 112.2 & 0 & 240 & $\ldots$ & & 0.37 & Malesani et al. (2016) \\
\hline $160625 B$ & 2016 Jun 25 22:40:16 & 488587220.275 & 308.57 & 6.93 & 0.07 & 41.2 & 40.1 & 1 & 1500 & 49 & & 1.41 & Xu et al. (2016) \\
\hline $160702 \mathrm{~A}$ & $2016 \mathrm{Jul} 02$ 12:22:24 & 489154948.000 & 231.09 & 9.07 & 0.21 & 72.0 & 59.2 & 0 & 31 & $\ldots$ & & $\ldots$ & \\
\hline 160709A & $2016 \mathrm{Jul} 09$ 19:49:03 & 489786547.510 & 236.11 & -28.51 & 0.27 & 47.6 & 82.2 & 0 & 210 & 15 & & $\ldots$ & \\
\hline $160816 \mathrm{~A}$ & 2016 Aug 16 17:30:57 & 493061461.980 & 322.32 & 37.14 & 0.23 & 33.4 & 18.3 & 1 & 190 & 7 & & $\ldots$ & \\
\hline $160821 \mathrm{~A}$ & 2016 Aug 21 20:34:30 & 493504474.040 & 171.29 & 42.29 & 0.12 & 19.0 & 66.9 & 1 & 220 & 31 & & $\ldots$ & \\
\hline $160829 \mathrm{~A}$ & 2016 Aug 29 08:01:37 & 494150501.650 & 201.72 & -56.78 & 0.10 & 14.5 & 56.9 & 1 & 30 & $\ldots$ & & $\ldots$ & \\
\hline $160905 \mathrm{~A}$ & 2016 Sep 05 11:18:55 & 494767139.910 & 162.01 & -50.88 & 0.10 & 17.1 & 57.5 & 1 & 140 & 22 & & $\ldots$ & \\
\hline $160910 \mathrm{~A}$ & 2016 Sep 10 17:19:39 & 495220783.160 & 221.31 & 38.81 & 0.59 & 71.4 & 79.0 & 1 & 38 & 20 & & $\ldots$ & \\
\hline 160917B & 2016 Sep 17 10:56:11 & 495802575.859 & 201.36 & 20.93 & 3.73 & 52.3 & 29.9 & 0 & $\ldots$ & 7 & FGGC & $\ldots$ & \\
\hline $160917 \mathrm{~A}$ & 2016 Sep 17 11:30:19 & 495804623.286 & 295.668 & 46.403 & 0.01 & 46.1 & 89.7 & 0 & $\ldots$ & 7 & GCN19927 & $\ldots$ & \\
\hline $161015 \mathrm{~A}$ & 2016 Oct 15 17:03:07 & 498243791.040 & 269.13 & 30.16 & 0.42 & 46.1 & 32.9 & 1 & 65 & $\ldots$ & & $\ldots$ & \\
\hline $161109 \mathrm{~A}$ & 2016 Nov 09 06:18:45 & 500365129.660 & 157.85 & 61.83 & 0.39 & 77.9 & 112.5 & 0 & 41 & $\ldots$ & & $\ldots$ & \\
\hline 170115B & 2017 Jan 15 17:49:14 & 506195359.030 & 189.32 & -46.63 & 0.74 & 36.0 & 42.5 & 1 & 67 & 13 & & $\cdots$ & \\
\hline $170127 \mathrm{C}$ & 2017 Jan 27 01:35:47 & 507173752.790 & 338.98 & -63.97 & 0.52 & 142.2 & 92.2 & 1 & 28 & $\cdots$ & & $\cdots$ & \\
\hline $170214 \mathrm{~A}$ & 2017 Feb 14 15:34:26 & 508779271.920 & 256.32 & -1.88 & 0.08 & 33.2 & 21.9 & 1 & 1100 & 46 & & 2.53 & Kruehler et al. (2017) \\
\hline $170228 \mathrm{~A}$ & 2017 Feb 28 19:03:00 & 510001385.710 & 239.66 & -6.49 & 2.02 & 65.1 & 32.0 & 0 & 31 & $\cdots$ & & $\cdots$ & \\
\hline 170306B & 2017 Mar 06 14:07:22 & 510502047.270 & 154.54 & 51.55 & 0.71 & 54.4 & 64.9 & 1 & 34 & $\ldots$ & & $\cdots$ & \\
\hline $170329 \mathrm{~A}$ & 2017 Mar 29 09:17:06 & 512471831.940 & 356.68 & 9.77 & 0.60 & 46.2 & 12.0 & 0 & 51 & $\cdots$ & & $\ldots$ & \\
\hline $170405 \mathrm{~A}$ & 2017 Apr 05 18:39:22 & 513110367.880 & 219.59 & -25.21 & 0.55 & 52.0 & 60.5 & 1 & 47 & 19 & & 3.51 & $\begin{array}{l}\text { de Ugarte Postigo et al. } \\
\text { (2017) }\end{array}$ \\
\hline $170409 \mathrm{~A}$ & 2017 Apr 09 02:42:00 & 513398525.490 & 347.53 & -7.17 & 0.10 & 85.9 & 66.6 & 1 & 47 & $\cdots$ & & $\cdots$ & \\
\hline $170424 \mathrm{~A}$ & 2017 Apr 24 10:12:30 & 514721555.760 & 343.7 & -45.12 & 0.69 & 57.4 & 76.6 & 0 & 34 & 12 & & $\cdots$ & \\
\hline $170510 \mathrm{~A}$ & 2017 May 10 05:12:25 & 516085950.730 & 160.21 & -39.28 & 0.49 & 66.4 & 23.5 & 1 & 35 & 8 & & $\cdots$ & \\
\hline $170522 \mathrm{~A}$ & 2017 May 22 15:45:35 & 517160740.290 & 139.35 & 25.67 & 0.23 & 49.4 & 74.5 & 1 & 69 & $\cdots$ & & $\cdots$ & \\
\hline 170728B & 2017 Jul 28 23:03:19 & 522975804.430 & 237.43 & 70.85 & 1.01 & 31.5 & 68.1 & 1 & 30 & 6 & & $\cdots$ & \\
\hline 170808B & 2017 Aug 08 22:27:43 & 523924068.100 & 145.72 & 1.61 & 0.71 & 64.8 & 17.5 & 1 & 46 & 7 & & $\cdots$ & \\
\hline $170813 \mathrm{~A}$ & 2017 Aug 13 01:13:08 & 524279593.800 & 200.45 & -5.49 & 0.75 & 41.1 & 22.6 & 0 & 27 & $\cdots$ & & $\cdots$ & \\
\hline 170825B & 2017 Aug 25 07:22:01 & 525338526.420 & 285.64 & -32.55 & 1.02 & 50.5 & 79.5 & 0 & 25 & $\cdots$ & & $\cdots$ & \\
\hline $170906 \mathrm{~A}$ & 2017 Sep 06 00:43:08 & 526351393.150 & 203.94 & -47.12 & 0.10 & 89.1 & 89.1 & 0 & 190 & $\cdots$ & & $\cdots$ & \\
\hline 170921B & 2017 Sep 21 04:02:11 & 527659336.510 & 123.93 & 52.67 & 0.26 & 104.6 & 78.2 & 0 & 28 & $\cdots$ & & $\cdots$ & \\
\hline 171010A & 2017 Oct 10 19:00:50 & 529354855.570 & 66.54 & -10.44 & 0.10 & 114.7 & 94.1 & 1 & 220 & $\cdots$ & & 0.33 & Kankare et al. (2017) \\
\hline $171011 \mathrm{C}$ & 2017 Oct 11 19:26:27 & 529442792.950 & 168.48 & 10.03 & 0.82 & 43.2 & 52.7 & 0 & 36 & $\cdots$ & & $\cdots$ & \\
\hline $171102 \mathrm{~A}$ & 2017 Nov 02 02:33:35 & 531282820.990 & 187.72 & 54.01 & 0.82 & 45.4 & 64.8 & 1 & 29 & $\cdots$ & & $\cdots$ & \\
\hline $171120 \mathrm{~A}$ & 2017 Nov 20 13:20:02 & 532876807.370 & 163.82 & 22.41 & 0.20 & 25.0 & 13.0 & 1 & 120 & $\cdots$ & & $\cdots$ & \\
\hline $171124 \mathrm{~A}$ & 2017 Nov 24 05:37:56 & 533194681.510 & 335.52 & 35.24 & 0.20 & 19.2 & 44.5 & 0 & 92 & $\ldots$ & & $\ldots$ & \\
\hline
\end{tabular}


Table 2

(Continued)

\begin{tabular}{|c|c|c|c|c|c|c|c|c|c|c|c|c|c|}
\hline GRB Name & $\begin{array}{l}\text { Date } \\
\text { (UT) }\end{array}$ & $\begin{array}{c}\text { Trigger Time } \\
\text { (MET) }\end{array}$ & $\begin{array}{l}\text { R.A. } \\
\text { (deg, } \\
\text { J2000) }\end{array}$ & $\begin{array}{c}\text { Decl. } \\
(\mathrm{deg}, \mathrm{J} 2000)\end{array}$ & $\begin{array}{l}\text { Loc. Err. } \\
\text { (deg) }\end{array}$ & $\begin{array}{c}\theta \\
(\operatorname{deg})\end{array}$ & $\begin{array}{c}\zeta \\
(\mathrm{deg})\end{array}$ & ARR & LTF TS & $\begin{array}{l}\text { LLE Sig. } \\
\qquad(\sigma)\end{array}$ & Loc. GCN & Redshift & $\mathrm{GCN}$ \\
\hline $171210 \mathrm{~A}$ & 2017 Dec 10 11:49:15 & 534599360.260 & 335.29 & 24.44 & 0.32 & 51.1 & 3.5 & 1 & 120 & 14 & & $\ldots$ & \\
\hline $171212 B$ & 2017 Dec 12 10:24:18 & 534767063.510 & 39.42 & -70.63 & 0.43 & 11.5 & 61.5 & 0 & 26 & $\ldots$ & & $\ldots$ & \\
\hline $180113 \mathrm{C}$ & 2018 Jan 13 10:02:05 & 537530530.407 & 174.63 & -64.74 & 1.00 & 62.6 & 108.9 & 1 & $\ldots$ & 40 & FGGC & $\ldots$ & \\
\hline $180210 \mathrm{~A}$ & 2018 Feb 10 12:24:38 & 539958283.550 & 1.83 & 18.49 & 0.12 & 34.2 & 20.0 & 1 & 160 & $\ldots$ & & $\ldots$ & \\
\hline $180305 \mathrm{~A}$ & 2018 Mar 05 09:26:08 & 541934773.660 & 49.67 & 32.1 & 0.11 & 74.9 & 89.7 & 0 & 29 & 8 & & $\ldots$ & \\
\hline $180526 \mathrm{~A}$ & 2018 May 26 11:04:09 & 549025454.000 & 108.48 & 3.64 & 0.27 & 82.2 & 109.7 & 0 & 46 & $\ldots$ & & $\ldots$ & \\
\hline $180703 \mathrm{~A}$ & 2018 Jul 03 21:01:40 & 552344505.490 & 6.56 & -67.1 & 0.33 & 44.0 & 94.0 & 0 & 54 & 18 & & $\ldots$ & \\
\hline 180703B & 2018 Jul 03 22:46:51 & 552350816.320 & 96.92 & -29.88 & 0.44 & 32.2 & 44.4 & 0 & 41 & $\ldots$ & & $\ldots$ & \\
\hline 180718B & 2018 Jul 18 18:18:24 & 553630709.460 & 44.48 & -30.33 & 0.95 & 46.5 & 43.1 & 0 & 37 & $\ldots$ & & $\ldots$ & \\
\hline 180720B & 2018 Jul 20 14:21:39 & 553789304.650 & 0.59 & -2.95 & 0.11 & 49.1 & 7.0 & 0 & 660 & 24 & & 0.65 & \\
\hline
\end{tabular}

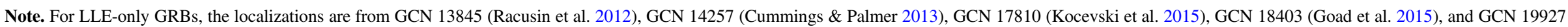
(Evans et al. 2016). 
Table 3

Comparison between Duration Estimators for the Detected Fermi-LAT GRBs

\begin{tabular}{|c|c|c|c|c|c|c|c|c|c|c|}
\hline GRB Name & Class & $\begin{array}{c}T_{\mathrm{GBM}, 05} \\
(\mathrm{~s})\end{array}$ & $\begin{array}{c}T_{\mathrm{GBM}, 95} \\
\text { (s) }\end{array}$ & $\begin{array}{c}T_{\mathrm{GBM}, 90} \\
\text { (s) }\end{array}$ & $\begin{array}{l}T_{\text {LLE, } 05} \\
(\mathrm{~s})\end{array}$ & $\begin{array}{l}T_{\text {LLE, } 95} \\
(\mathrm{~s})\end{array}$ & $\begin{array}{l}T_{\text {LLE, } 90} \\
(\mathrm{~s})\end{array}$ & $\begin{array}{l}T_{\mathrm{LAT}, 0} \\
(\mathrm{~s})\end{array}$ & $\begin{array}{l}T_{\mathrm{LAT}, 1} \\
(\mathrm{~s})\end{array}$ & $\begin{array}{c}T_{\mathrm{LAT}, 100} \\
(\mathrm{~s})\end{array}$ \\
\hline 080818B & (L) & -0.51 & 12.86 & 13.38 & $\ldots$ & $\ldots$ & $\ldots$ & 9018.9 & 9482.8 & $500 \pm 200$ \\
\hline 080825C & (L) & 1.2 & 22.21 & 20.99 & 2.84 & 4.05 & 1.20 & 3.1 & 173.5 & $170.4 \pm 0.7$ \\
\hline 080916C & (L) & 1.3 & 64.26 & 62.98 & 1.00 & 46.55 & 45.55 & 3.0 & 1531.8 & $1500 \pm 200$ \\
\hline 081006 & (L) & -0.26 & 6.14 & 6.40 & $\ldots$ & $\ldots$ & $\ldots$ & 0.8 & 62.3 & $60 \pm 20$ \\
\hline 081009 & (L) & 1.3 & 42.69 & 41.34 & $\ldots$ & $\ldots$ & $\ldots$ & 67.8 & 1250.1 & $1200 \pm 200$ \\
\hline 081024B & (S) & -0.064 & 0.58 & 0.64 & -0.04 & 1.28 & 1.32 & 0.2 & 2.2 & $2.02 \pm 0.04$ \\
\hline 081102B & (S) & -0.064 & 1.66 & 1.73 & $\ldots$ & $\ldots$ & $\ldots$ & 0.3 & 0.4 & $0.15 \pm 0.03$ \\
\hline 081122A & (L) & -0.26 & 23.04 & 23.30 & $\ldots$ & $\ldots$ & $\ldots$ & 23.5 & 66.5 & $40 \pm 20$ \\
\hline 081203A & (L) & * & 214.00 & 214.00 & $\ldots$ & $\ldots$ & $\ldots$ & 291.9 & 379.7 & $90 \pm 10$ \\
\hline 081224 & (L) & 0.74 & 17.18 & 16.45 & $\ldots$ & $\ldots$ & $\ldots$ & 76.5 & 339.2 & $260 \pm 40$ \\
\hline 090102 & (L) & 1.5 & 28.16 & 26.62 & $\ldots$ & $\ldots$ & $\ldots$ & 3915.9 & 4404.8 & $500 \pm 300$ \\
\hline 090217 & (L) & 0.83 & 34.11 & 33.28 & 1.96 & 15.50 & 13.54 & 4.1 & 71.6 & $68 \pm 10$ \\
\hline 090227A & (L) & 0.003 & 16.19 & 16.19 & 1.15 & 11.05 & 9.90 & 2.9 & 204.3 & $201.4 \pm 0.2$ \\
\hline 090227B & (S) & -0.016 & 0.29 & 0.30 & 0.04 & 1.18 & 1.14 & $\cdots$ & $\ldots$ & $\ldots$ \\
\hline 090228A & (S) & * & 0.45 & 0.45 & $\ldots$ & $\ldots$ & $\ldots$ & 0.1 & 2.1 & $2.0 \pm 0.1$ \\
\hline 090323 & (L) & 8.7 & 142.59 & 133.89 & 7.24 & 93.41 & 86.18 & 9.3 & 5321.6 & $5312 \pm 8$ \\
\hline 090328 & (L) & 4.4 & 66.05 & 61.70 & 8.05 & 37.02 & 28.97 & 14.4 & 6150.6 & $6140 \pm 70$ \\
\hline 090427A & (L) & $*$ & 15.00 & 15.00 & $\ldots$ & $\ldots$ & $\ldots$ & 422.9 & 435.6 & $10 \pm 10$ \\
\hline 090510 & (S) & -0.048 & 0.91 & 0.96 & 0.54 & 5.93 & 5.40 & 0.0 & 170.0 & $170 \pm 2$ \\
\hline 090531B & (S) & * & 0.77 & 0.77 & -0.07 & 0.00 & 0.07 & $\ldots$ & $\ldots$ & $\ldots$ \\
\hline 090626 & (L) & 1.5 & 50.43 & 48.90 & 21.48 & 22.37 & 0.89 & 9.0 & 557.3 & $550 \pm 90$ \\
\hline 090720B & (L) & -0.26 & 10.50 & 10.75 & $\ldots$ & $\ldots$ & $\ldots$ & 0.1 & 1.7 & $1.6 \pm 0.4$ \\
\hline 090902B & (L) & 2.8 & 22.14 & 19.33 & -0.65 & 17.23 & 17.88 & 0.5 & 884.2 & $880 \pm 60$ \\
\hline 090926A & (L) & 2.2 & 15.94 & 13.76 & 1.15 & 16.71 & 15.56 & 2.2 & 4419.5 & $4420 \pm 50$ \\
\hline 091003 & (L) & 0.83 & 21.06 & 20.22 & $\ldots$ & $\ldots$ & $\ldots$ & 1.0 & 392.0 & $390 \pm 10$ \\
\hline 091031 & (L) & 1.4 & 35.33 & 33.92 & -1.53 & 15.09 & 16.62 & 0.1 & 408.2 & $410 \pm 30$ \\
\hline 091120 & (L) & 1.0 & 51.20 & 50.18 & $\ldots$ & $\ldots$ & $\ldots$ & 31.8 & 803.9 & $770 \pm 20$ \\
\hline 091127 & (L) & 0.003 & 8.70 & 8.70 & $\ldots$ & $\ldots$ & $\ldots$ & 8.6 & 35.4 & $30 \pm 10$ \\
\hline $100116 \mathrm{~A}$ & (L) & 0.58 & 103.11 & 102.53 & 89.94 & 96.59 & 6.66 & 77.5 & 730.4 & $650 \pm 50$ \\
\hline $100213 C$ & (L) & * & 60.00 & 60.00 & $\ldots$ & $\ldots$ & $\ldots$ & 2707.2 & 3389.0 & $700 \pm 300$ \\
\hline $100225 \mathrm{~A}$ & (L) & -0.26 & 12.74 & 12.99 & 2.75 & 9.74 & 6.99 & 6.6 & 1012.4 & $1010 \pm 20$ \\
\hline $100325 \mathrm{~A}$ & (L) & -0.38 & 6.72 & 7.10 & $\ldots$ & $\ldots$ & $\ldots$ & 0.2 & 1.2 & $0.9 \pm 0.2$ \\
\hline $100414 \mathrm{~A}$ & (L) & 1.9 & 28.35 & 26.50 & $\ldots$ & $\ldots$ & $\ldots$ & 18.7 & 5506.1 & $5490 \pm 30$ \\
\hline 100423B & (L) & 1.6 & 18.11 & 16.51 & $\ldots$ & $\ldots$ & $\ldots$ & 166.7 & 180.6 & $14 \pm 7$ \\
\hline $100511 \mathrm{~A}$ & (L) & 0.83 & 43.27 & 42.43 & $\ldots$ & $\ldots$ & $\ldots$ & 11.6 & 6338.2 & $6300 \pm 700$ \\
\hline $100620 \mathrm{~A}$ & (L) & 0.19 & 52.03 & 51.84 & $\ldots$ & $\ldots$ & $\ldots$ & 3.8 & 42.8 & $39 \pm 6$ \\
\hline 100724B & (L) & 8.2 & 122.88 & 114.69 & 2.63 & 102.44 & 99.81 & 9.1 & 53.6 & $45 \pm 6$ \\
\hline $100728 \mathrm{~A}$ & (L) & 13 & 178.69 & 165.38 & $\ldots$ & $\ldots$ & $\ldots$ & 248.6 & 1340.5 & $1090 \pm 40$ \\
\hline $100826 \mathrm{~A}$ & (L) & 8.7 & 93.70 & 84.99 & 18.20 & 39.28 & 21.09 & 33.5 & 61.4 & $28 \pm 6$ \\
\hline $101014 \mathrm{~A}$ & (L) & 1.4 & 450.82 & 449.42 & 208.72 & 210.38 & 1.67 & 2270.7 & 4196.0 & $1930 \pm 50$ \\
\hline $101107 \mathrm{~A}$ & (L) & 2.3 & 378.12 & 375.81 & $\ldots$ & $\ldots$ & $\ldots$ & 134.0 & 239.4 & $105 \pm 4$ \\
\hline $101123 \mathrm{~A}$ & (L) & 41 & 145.41 & 103.94 & 43.43 & 46.13 & 2.70 & $\ldots$ & $\ldots$ & $\ldots$ \\
\hline $101227 \mathrm{~B}$ & (L) & 0.77 & 154.12 & 153.35 & $\ldots$ & $\ldots$ & $\ldots$ & 17.3 & 23.8 & $7 \pm 3$ \\
\hline $110120 \mathrm{~A}$ & (L) & 0.003 & 26.18 & 26.17 & 0.29 & 0.53 & 0.23 & 0.6 & 1112.8 & $1100 \pm 200$ \\
\hline $110123 \mathrm{~A}$ & (L) & 0.70 & 18.56 & 17.86 & $\ldots$ & $\ldots$ & $\ldots$ & 51.6 & 527.0 & $480 \pm 20$ \\
\hline $110213 \mathrm{~A}$ & (L) & -0.77 & 33.54 & 34.30 & $\ldots$ & $\ldots$ & $\ldots$ & 1261.4 & 1944.2 & $700 \pm 400$ \\
\hline 110328B & (L) & 2.6 & 86.53 & 83.97 & -3.05 & 23.23 & 26.27 & 102.5 & 737.2 & $630 \pm 60$ \\
\hline $110428 \mathrm{~A}$ & (L) & 2.7 & 8.32 & 5.63 & $\ldots$ & $\ldots$ & $\ldots$ & 7.3 & 393.5 & $386.2 \pm 0.3$ \\
\hline $110518 \mathrm{~A}$ & (L) & * & 35.00 & 35.00 & $\ldots$ & $\ldots$ & $\ldots$ & 1968.2 & 2364.2 & $400 \pm 200$ \\
\hline $110529 \mathrm{~A}$ & (S) & -0.13 & 0.38 & 0.51 & 0.01 & 0.11 & 0.09 & $\ldots$ & $\ldots$ & .. \\
\hline $110625 \mathrm{~A}$ & (L) & 3.8 & 30.72 & 26.88 & $\ldots$ & $\ldots$ & $\ldots$ & 205.9 & 577.2 & $400 \pm 100$ \\
\hline $110721 \mathrm{~A}$ & (L) & 0.003 & 21.82 & 21.82 & -0.75 & 5.25 & 6.00 & 0.0 & 120.6 & $121 \pm 3$ \\
\hline $110728 \mathrm{~A}$ & (S) & -0.13 & 0.58 & 0.70 & $\ldots$ & $\ldots$ & $\ldots$ & 0.4 & 3.0 & $2.6 \pm 0.9$ \\
\hline $110731 \mathrm{~A}$ & (L) & 0.003 & 7.49 & 7.49 & 2.11 & 8.37 & 6.26 & 1.1 & 436.0 & $430 \pm 10$ \\
\hline $110903 \mathrm{~A}$ & (L) & -0.26 & 341.00 & 341.25 & $\ldots$ & $\ldots$ & $\ldots$ & 46.7 & 370.1 & $320 \pm 50$ \\
\hline 110921B & (L) & 0.90 & 18.56 & 17.66 & 17.07 & 18.01 & 0.93 & 7.4 & 321.6 & $310 \pm 50$ \\
\hline $111210 \mathrm{~B}$ & (L) & $*$ & 60.00 & 60.00 & $\ldots$ & $\ldots$ & $\ldots$ & 6.5 & 394.0 & $390 \pm 70$ \\
\hline $120107 \mathrm{~A}$ & (L) & 0.064 & 23.10 & 23.04 & $\ldots$ & $\ldots$ & $\ldots$ & 1.3 & 48.8 & $50 \pm 10$ \\
\hline $120226 \mathrm{~A}$ & (L) & 4.4 & 57.34 & 52.99 & 4.46 & 33.38 & 28.93 & 29.4 & 283.6 & $250 \pm 20$ \\
\hline $120316 \mathrm{~A}$ & (L) & 1.5 & 28.16 & 26.62 & 11.31 & 11.71 & 0.40 & 15.0 & 545.0 & $530 \pm 30$ \\
\hline $120328 \mathrm{~B}$ & (L) & 3.8 & 33.54 & 29.70 & 4.51 & 7.25 & 2.74 & $\ldots$ & $\ldots$ & $\ldots$ \\
\hline 120420B & (L) & 0.003 & 254.92 & 254.91 & $\ldots$ & $\ldots$ & $\ldots$ & 3501.7 & 3908.0 & $410 \pm 10$ \\
\hline $120526 \mathrm{~A}$ & (L) & 3.1 & 46.72 & 43.65 & $\ldots$ & $\ldots$ & $\ldots$ & 692.2 & 3306.3 & $2600 \pm 200$ \\
\hline
\end{tabular}


Table 3

(Continued)

\begin{tabular}{|c|c|c|c|c|c|c|c|c|c|c|}
\hline GRB Name & Class & $\underset{\text { (s) }}{T_{\mathrm{GBM}, 05}}$ & $\underset{(\mathrm{s})}{T_{\mathrm{GBM}, 95}}$ & $\underset{\text { (s) }}{T_{\mathrm{GBM}, 90}}$ & $\begin{array}{l}T_{\mathrm{LLE}, 05} \\
(\mathrm{~s})\end{array}$ & $\begin{array}{c}T_{\text {LLE, } 95} \\
(\mathrm{~s})\end{array}$ & $\begin{array}{c}T_{\text {LLE, } 90} \\
(\mathrm{~s})\end{array}$ & $\begin{array}{l}T_{\mathrm{LAT}, 0} \\
(\mathrm{~s})\end{array}$ & $\begin{array}{l}T_{\mathrm{LAT}, 1} \\
(\mathrm{~s})\end{array}$ & $\begin{array}{l}T_{\mathrm{LAT}, 100} \\
(\mathrm{~s})\end{array}$ \\
\hline $120624 B$ & (L) & -260 & 14.34 & 271.36 & 5.76 & 9.12 & 3.36 & 73.7 & 1103.9 & $1030 \pm 30$ \\
\hline 120709A & (L) & -0.13 & 27.20 & 27.33 & -0.12 & 0.34 & 0.46 & 0.1 & 695.9 & $700 \pm 10$ \\
\hline 120711A & (L) & 62 & 106.50 & 44.03 & $\ldots$ & $\ldots$ & $\ldots$ & 393.3 & 5431.6 & $5000 \pm 800$ \\
\hline $120729 \mathrm{~A}$ & (L) & -1.0 & 24.45 & 25.47 & $\ldots$ & $\ldots$ & $\ldots$ & 396.9 & 432.0 & $40 \pm 10$ \\
\hline $120830 \mathrm{~A}$ & (S) & $*$ & 0.90 & 0.90 & $\ldots$ & $\ldots$ & $\ldots$ & 0.7 & 10.7 & $10.0 \pm 0.2$ \\
\hline 120911B & (L) & * & 69.00 & 69.00 & 7.14 & 35.37 & 28.23 & 9.2 & 217.8 & $209 \pm 2$ \\
\hline $120915 \mathrm{~A}$ & (S) & -0.32 & 0.26 & 0.58 & $\ldots$ & $\ldots$ & $\ldots$ & 0.2 & 0.8 & $0.6 \pm 0.3$ \\
\hline 120919B & (L) & 2.0 & 120.07 & 118.02 & $\ldots$ & $\ldots$ & $\ldots$ & 121.2 & 605.3 & $500 \pm 200$ \\
\hline $121011 \mathrm{~A}$ & (L) & 1.0 & 66.82 & 65.79 & -0.63 & 7.21 & 7.84 & $\ldots$ & $\ldots$ & $\ldots$ \\
\hline $121029 \mathrm{~A}$ & (L) & -0.90 & 14.91 & 15.81 & $\ldots$ & $\ldots$ & $\ldots$ & 119.0 & 1926.5 & $1810 \pm 70$ \\
\hline 121123B & (L) & 2.3 & 44.80 & 42.50 & $\ldots$ & $\ldots$ & $\ldots$ & 268.0 & 1651.3 & $1400 \pm 200$ \\
\hline $121225 B$ & (L) & 9.5 & 67.97 & 58.50 & 16.90 & 21.11 & 4.21 & $\ldots$ & $\ldots$ & $\ldots$ \\
\hline $130305 \mathrm{~A}$ & (L) & 1.3 & 26.88 & 25.60 & 5.31 & 22.09 & 16.78 & $\ldots$ & $\ldots$ & $\ldots$ \\
\hline $130310 \mathrm{~A}$ & (L) & 4.1 & 20.10 & 16.00 & 4.13 & 4.21 & 0.08 & 67.2 & 560.1 & $490 \pm 30$ \\
\hline $130325 \mathrm{~A}$ & (L) & 0.77 & 7.68 & 6.91 & $\ldots$ & $\ldots$ & $\ldots$ & 324.1 & 1029.7 & $700 \pm 200$ \\
\hline $130327 \mathrm{~B}$ & (L) & 2.0 & 33.28 & 31.23 & $\ldots$ & $\ldots$ & $\ldots$ & 8.0 & 523.2 & $520 \pm 10$ \\
\hline 130427A & (L) & 4.1 & 142.34 & 138.24 & -0.12 & 46.19 & 46.30 & 0.1 & 34366.2 & $34400 \pm 300$ \\
\hline $130502 B$ & (L) & 7.2 & 31.49 & 24.32 & 13.80 & 15.37 & 1.56 & 12.4 & 1316.6 & $1300 \pm 200$ \\
\hline $130504 \mathrm{C}$ & (L) & 8.7 & 81.92 & 73.22 & 13.54 & 70.90 & 57.36 & 42.6 & 590.3 & $550 \pm 20$ \\
\hline $130518 \mathrm{~A}$ & (L) & 9.9 & 58.50 & 48.58 & 23.00 & 37.71 & 14.72 & 26.8 & 343.6 & $320 \pm 10$ \\
\hline 130606B & (L) & 5.4 & 57.60 & 52.23 & $\ldots$ & $\ldots$ & $\ldots$ & 130.5 & 527.3 & $400 \pm 40$ \\
\hline $130702 \mathrm{~A}$ & (L) & 0.77 & 59.65 & 58.88 & $\ldots$ & $\ldots$ & $\ldots$ & 272.3 & 1283.3 & $1000 \pm 100$ \\
\hline $130821 \mathrm{~A}$ & (L) & 3.6 & 90.62 & 87.04 & 24.79 & 34.07 & 9.28 & 33.9 & 6103.7 & $6000 \pm 2000$ \\
\hline $130828 \mathrm{~A}$ & (L) & 13 & 150.27 & 136.96 & 35.08 & 68.78 & 33.70 & 17.1 & 616.8 & $600 \pm 10$ \\
\hline $130907 \mathrm{~A}$ & (L) & * & 210.00 & 210.00 & $\ldots$ & $\ldots$ & $\ldots$ & 3618.4 & 4010.9 & $400 \pm 300$ \\
\hline $131014 \mathrm{~A}$ & (L) & 0.96 & 4.16 & 3.20 & 1.68 & 3.49 & 1.81 & 1.9 & 200.4 & $199 \pm 5$ \\
\hline 131029A & (L) & 1.0 & 105.47 & 104.45 & $\ldots$ & $\ldots$ & $\ldots$ & 38.7 & 557.2 & $520 \pm 50$ \\
\hline $131108 \mathrm{~A}$ & (L) & 0.32 & 18.50 & 18.18 & 0.04 & 10.99 & 10.96 & 0.0 & 678.1 & $680 \pm 40$ \\
\hline $131209 \mathrm{~A}$ & (L) & 2.8 & 16.38 & 13.57 & $\ldots$ & $\ldots$ & $\ldots$ & 14.3 & 374.8 & $360 \pm 60$ \\
\hline $131216 \mathrm{~A}$ & (L) & 0.003 & 19.27 & 19.26 & -0.19 & 0.09 & 0.28 & $\cdots$ & $\ldots$ & $\ldots$ \\
\hline $131231 \mathrm{~A}$ & (L) & 13 & 44.54 & 31.23 & 21.45 & 34.25 & 12.80 & 23.1 & 4824.2 & $4800 \pm 700$ \\
\hline $140102 \mathrm{~A}$ & (L) & 0.45 & 4.10 & 3.65 & 2.38 & 5.25 & 2.87 & 3.1 & 60.2 & $60 \pm 10$ \\
\hline 140104B & (L) & 11 & 198.15 & 187.14 & $\ldots$ & $\ldots$ & $\ldots$ & 227.3 & 1174.0 & $900 \pm 200$ \\
\hline 140110A & (L) & -0.26 & 9.22 & 9.47 & 0.15 & 6.13 & 5.98 & 0.6 & 159.4 & $159 \pm 8$ \\
\hline 140124A & (L) & -13 & 108.87 & 121.54 & $\ldots$ & $\ldots$ & $\ldots$ & 93.6 & 468.2 & $370 \pm 90$ \\
\hline $140206 \mathrm{~B}$ & (L) & 7.5 & 154.18 & 146.69 & 5.88 & 33.48 & 27.60 & 0.6 & 8585.0 & $8584.43 \pm 0.01$ \\
\hline 140219A & (L) & -2.6 & 74.50 & 77.06 & $\ldots$ & $\ldots$ & $\ldots$ & 959.8 & 1802.0 & $840 \pm 50$ \\
\hline $140323 \mathrm{~A}$ & (L) & 5.1 & 116.48 & 111.43 & $\ldots$ & $\ldots$ & $\ldots$ & 226.8 & 715.6 & $500 \pm 100$ \\
\hline $140402 \mathrm{~A}$ & (S) & -0.13 & 0.19 & 0.32 & -0.31 & 1.24 & 1.55 & 0.1 & 71.7 & $72 \pm 5$ \\
\hline 140416A & (L) & -2.8 & 28.96 & 31.74 & $\ldots$ & $\ldots$ & $\ldots$ & 1174.8 & 2207.4 & $1000 \pm 300$ \\
\hline $140523 \mathrm{~A}$ & (L) & 0.58 & 19.78 & 19.20 & $\ldots$ & $\ldots$ & $\ldots$ & 1.6 & 470.2 & $470 \pm 20$ \\
\hline $140528 \mathrm{~A}$ & (L) & 1.0 & 14.59 & 13.57 & $\ldots$ & $\ldots$ & $\ldots$ & 81.1 & 1377.9 & $1300 \pm 300$ \\
\hline 140619B & (L) & -0.26 & 2.56 & 2.82 & 0.17 & 1.30 & 1.12 & 0.2 & 5.3 & $5 \pm 1$ \\
\hline $140723 \mathrm{~A}$ & (L) & $*$ & 56.32 & 56.32 & -0.31 & 20.51 & 20.82 & 0.6 & 103.8 & $103 \pm 1$ \\
\hline $140729 \mathrm{~A}$ & (L) & 0.51 & 56.06 & 55.55 & $\ldots$ & $\ldots$ & $\ldots$ & 4.8 & 73.4 & $68.6 \pm 0.4$ \\
\hline $140810 \mathrm{~A}$ & (L) & 6.7 & 88.32 & 81.67 & $\ldots$ & $\ldots$ & $\ldots$ & 460.8 & 18827.5 & $18400 \pm 100$ \\
\hline $140825 \mathrm{~A}$ & (L) & $*$ & 14.00 & 14.00 & $\ldots$ & $\ldots$ & $\ldots$ & 1463.0 & 1702.3 & $200 \pm 100$ \\
\hline $140928 \mathrm{~A}$ & (L) & -11 & 7.17 & 17.92 & $\ldots$ & $\ldots$ & $\ldots$ & 1676.6 & 2554.7 & $880 \pm 40$ \\
\hline $141012 \mathrm{~A}$ & (L) & -26 & 11.78 & 37.63 & $\ldots$ & $\ldots$ & $\ldots$ & 3.1 & 107.0 & $100 \pm 10$ \\
\hline $141028 \mathrm{~A}$ & (L) & 6.7 & 38.15 & 31.49 & 6.86 & 30.75 & 23.89 & 10.9 & 500.5 & $490 \pm 80$ \\
\hline $141102 \mathrm{~A}$ & (L) & -0.064 & 2.56 & 2.62 & $\ldots$ & $\ldots$ & $\ldots$ & 3.5 & 57.7 & $54 \pm 1$ \\
\hline $141113 \mathrm{~A}$ & (S) & -0.064 & 0.38 & 0.45 & $\ldots$ & $\ldots$ & $\ldots$ & 0.1 & 3.5 & $3.4 \pm 0.1$ \\
\hline $141207 \mathrm{~A}$ & (L) & 1.3 & 22.27 & 20.99 & 1.69 & 12.17 & 10.48 & 1.8 & 734.3 & $730 \pm 10$ \\
\hline $141221 \mathrm{~B}$ & (L) & -1.3 & 31.23 & 32.51 & $\ldots$ & $\ldots$ & ... & 22.2 & 58.5 & $36 \pm 7$ \\
\hline $141222 \mathrm{~A}$ & (L) & $*$ & 2.75 & 2.75 & -0.03 & 1.26 & 1.29 & 34.2 & 440.6 & $410 \pm 10$ \\
\hline 150118B & (L) & 7.7 & 47.87 & 40.19 & 22.12 & 23.59 & 1.47 & 4.3 & 51.0 & $50 \pm 20$ \\
\hline $150202 B$ & (L) & 1.5 & 168.96 & 167.43 & 4.86 & 17.51 & 12.65 & 44.9 & 115.7 & $70 \pm 50$ \\
\hline $150210 \mathrm{~A}$ & (L) & 0.003 & 31.30 & 31.29 & 0.03 & 2.55 & 2.52 & 0.8 & 169.4 & $170 \pm 20$ \\
\hline $150314 \mathrm{~A}$ & (L) & 0.61 & 11.30 & 10.69 & -0.17 & 0.03 & 0.20 & 0.1 & 3064.3 & $3100 \pm 100$ \\
\hline $150403 \mathrm{~A}$ & (L) & 3.3 & 25.60 & 22.27 & 9.97 & 14.08 & 4.11 & 399.5 & 970.5 & $600 \pm 200$ \\
\hline $150416 \mathrm{~A}$ & (L) & 0.51 & 33.79 & 33.28 & -3.28 & 19.07 & 22.36 & $\ldots$ & $\ldots$ & .. \\
\hline $150510 \mathrm{~A}$ & (L) & 0.38 & 52.29 & 51.90 & 0.03 & 16.41 & 16.38 & 2.4 & 172.5 & $170 \pm 10$ \\
\hline $150513 \mathrm{~A}$ & (L) & -160 & 1.79 & 158.98 & -157.75 & -146.39 & 11.36 & $\ldots$ & $\ldots$ & $\ldots$ \\
\hline
\end{tabular}


Table 3

(Continued)

\begin{tabular}{|c|c|c|c|c|c|c|c|c|c|c|}
\hline GRB Name & Class & $\begin{array}{c}T_{\mathrm{GBM}, 05} \\
\text { (s) }\end{array}$ & $\underset{\text { (s) }}{T_{\mathrm{GBM}, 95}}$ & $\underset{\text { (s) }}{T_{\mathrm{GBM}, 90}}$ & $\begin{array}{l}T_{\mathrm{LLE}, 05} \\
\text { (s) }\end{array}$ & $\begin{array}{l}T_{\text {LLE,95 }} \\
\text { (s) }\end{array}$ & $\begin{array}{l}T_{\text {LLE, } 90} \\
(\mathrm{~s})\end{array}$ & $\begin{array}{l}T_{\mathrm{LAT}, 0} \\
(\mathrm{~s})\end{array}$ & $\begin{array}{l}T_{\mathrm{LAT}, 1} \\
(\mathrm{~s})\end{array}$ & $\begin{array}{c}T_{\mathrm{LAT}, 100} \\
(\mathrm{~s})\end{array}$ \\
\hline $150514 \mathrm{~A}$ & (L) & 0.003 & 10.82 & 10.81 & $\ldots$ & $\ldots$ & $\ldots$ & 442.4 & 597.6 & $200 \pm 200$ \\
\hline $150523 \mathrm{~A}$ & (L) & 1.8 & 84.22 & 82.43 & 2.92 & 43.39 & 40.48 & 3.9 & 6129.1 & $6130 \pm 80$ \\
\hline $150627 \mathrm{~A}$ & (L) & 5.3 & 69.89 & 64.58 & 4.51 & 18.09 & 13.58 & 152.0 & 6143.0 & $6000 \pm 1000$ \\
\hline $150702 \mathrm{~A}$ & (L) & 1.3 & 47.17 & 45.83 & $\ldots$ & $\ldots$ & $\ldots$ & 512.6 & 1853.3 & $1340 \pm 20$ \\
\hline $150820 \mathrm{~A}$ & (L) & -0.77 & 5.12 & 5.89 & -1.06 & -0.64 & 0.42 & $\ldots$ & $\ldots$ & $\ldots$ \\
\hline $150902 \mathrm{~A}$ & (L) & 3.8 & 17.41 & 13.57 & 3.02 & 12.61 & 9.60 & 3.5 & 408.7 & $405 \pm 2$ \\
\hline $151006 \mathrm{~A}$ & (L) & 1.5 & 94.98 & 93.44 & -1.12 & 15.02 & 16.14 & $\ldots$ & $\ldots$ & $\ldots$ \\
\hline 160101B & (L) & 0.003 & 22.02 & 22.01 & -1.26 & 3.50 & 4.76 & $\ldots$ & $\ldots$ & $\ldots$ \\
\hline $160310 \mathrm{~A}$ & (L) & -0.26 & 25.60 & 25.86 & $\ldots$ & $\ldots$ & $\ldots$ & 99.2 & 432.2 & $300 \pm 200$ \\
\hline $160314 \mathrm{~B}$ & (L) & -3.8 & 94.72 & 98.56 & $\ldots$ & $\ldots$ & $\ldots$ & 133.4 & 1285.4 & $1200 \pm 200$ \\
\hline $160325 \mathrm{~A}$ & (L) & 2.0 & 44.99 & 42.95 & 13.82 & 14.02 & 0.20 & 5.0 & 1555.7 & $1600 \pm 500$ \\
\hline $160422 \mathrm{~A}$ & (L) & 0.83 & 13.12 & 12.29 & $\ldots$ & $\ldots$ & $\ldots$ & 769.6 & 1061.4 & $300 \pm 300$ \\
\hline $160503 \mathrm{~A}$ & (L) & -2.6 & 26.11 & 28.67 & $\ldots$ & $\ldots$ & $\ldots$ & 5324.7 & 23075.4 & $17750 \pm 70$ \\
\hline $160509 \mathrm{~A}$ & (L) & 8.2 & 377.86 & 369.67 & 8.81 & 25.12 & 16.31 & 9.6 & 5687.4 & $6000 \pm 1000$ \\
\hline $160521 \mathrm{~B}$ & (L) & 0.32 & 3.14 & 2.82 & $\ldots$ & $\ldots$ & $\ldots$ & 90.2 & 2199.4 & $2100 \pm 300$ \\
\hline $160623 \mathrm{~A}$ & (L) & -1.3 & 106.50 & 107.78 & $\ldots$ & $\ldots$ & $\ldots$ & 401.5 & 35069.0 & $35000 \pm 1000$ \\
\hline $160625 \mathrm{~B}$ & (L) & 190 & 641.84 & 453.38 & 184.86 & 220.27 & 35.41 & 25.6 & 840.5 & $810 \pm 20$ \\
\hline $160702 \mathrm{~A}$ & (S) & * & 0.20 & 0.20 & $\ldots$ & $\ldots$ & $\ldots$ & 1941.5 & 2215.6 & $300 \pm 100$ \\
\hline $160709 \mathrm{~A}$ & (L) & * & 5.44 & 5.44 & 0.41 & 3.62 & 3.21 & 0.5 & 25.8 & $25 \pm 2$ \\
\hline $160816 \mathrm{~A}$ & (L) & 0.38 & 11.46 & 11.07 & 0.45 & 10.91 & 10.46 & 1.1 & 1094.8 & $1090 \pm 70$ \\
\hline $160821 \mathrm{~A}$ & (L) & 120 & 161.54 & 43.01 & 117.29 & 152.21 & 34.92 & 92.1 & 1459.2 & $1370 \pm 40$ \\
\hline $160829 A$ & (S) & -0.064 & 0.45 & 0.51 & $\ldots$ & $\ldots$ & $\ldots$ & 0.9 & 31.7 & $31 \pm 6$ \\
\hline $160905 \mathrm{~A}$ & (L) & 3.8 & 37.38 & 33.54 & 5.57 & 28.92 & 23.34 & 7.8 & 463.3 & $460 \pm 20$ \\
\hline $160910 \mathrm{~A}$ & (L) & 4.6 & 28.93 & 24.32 & 7.70 & 18.47 & 10.78 & 86.4 & 216.4 & $130 \pm 50$ \\
\hline 160917B & (L) & -4.6 & 9.98 & 14.59 & -0.90 & 5.55 & 6.45 & $\ldots$ & $\ldots$ & $\ldots$ \\
\hline $160917 \mathrm{~A}$ & (L) & -0.26 & 19.20 & 19.46 & -0.12 & 0.33 & 0.44 & $\ldots$ & $\ldots$ & $\ldots$ \\
\hline $161015 \mathrm{~A}$ & (L) & 0.26 & 15.36 & 15.10 & $\ldots$ & $\ldots$ & $\ldots$ & 1.1 & 7.5 & $6.4 \pm 0.2$ \\
\hline $161109 \mathrm{~A}$ & (L) & 3.8 & 27.39 & 23.55 & $\ldots$ & $\ldots$ & $\ldots$ & 423.4 & 890.9 & $500 \pm 200$ \\
\hline 170115B & (L) & 0.51 & 44.80 & 44.29 & 0.55 & 11.58 & 11.03 & 1.3 & 1027.9 & $1027 \pm 10$ \\
\hline $170127 \mathrm{C}$ & (S) & $*$ & 0.13 & 0.13 & $\ldots$ & $\ldots$ & $\ldots$ & 664.8 & 2889.0 & $2220 \pm 50$ \\
\hline $170214 \mathrm{~A}$ & (L) & 13 & 135.43 & 122.88 & 6.54 & 84.10 & 77.56 & 39.5 & 752.0 & $713 \pm 8$ \\
\hline $170228 \mathrm{~A}$ & (L) & 1.6 & 62.24 & 60.67 & $\ldots$ & $\ldots$ & $\ldots$ & 6.5 & 72.4 & $70 \pm 10$ \\
\hline 170306B & (L) & 4.6 & 23.55 & 18.94 & $\ldots$ & $\ldots$ & $\ldots$ & 21.2 & 71.5 & $50 \pm 10$ \\
\hline $170329 \mathrm{~A}$ & (L) & 0.26 & 33.79 & 33.54 & $\ldots$ & $\ldots$ & $\ldots$ & 3.9 & 52.4 & $48 \pm 7$ \\
\hline $170405 \mathrm{~A}$ & (L) & 7.4 & 86.02 & 78.59 & 16.56 & 51.51 & 34.95 & 17.8 & 868.0 & $850 \pm 60$ \\
\hline $170409 \mathrm{~A}$ & (L) & 29 & 93.44 & 64.00 & $\ldots$ & $\ldots$ & $\ldots$ & 178.4 & 440.3 & $260 \pm 30$ \\
\hline $170424 \mathrm{~A}$ & (L) & 2.8 & 56.06 & 53.25 & 2.42 & 37.62 & 35.19 & 21.8 & 107.7 & $86 \pm 2$ \\
\hline $170510 \mathrm{~A}$ & (L) & 2.8 & 130.56 & 127.75 & -1.03 & 27.68 & 28.71 & 26.8 & 154.2 & $127 \pm 5$ \\
\hline $170522 \mathrm{~A}$ & (L) & 0.58 & 8.00 & 7.42 & $\ldots$ & $\ldots$ & $\ldots$ & 2.8 & 41.6 & $40 \pm 10$ \\
\hline 170728B & (L) & * & 46.34 & 46.34 & -0.01 & 0.40 & 0.41 & 0.2 & 469.3 & $469.070 \pm 0.010$ \\
\hline 170808B & (L) & 4.1 & 21.76 & 17.66 & 6.47 & 16.77 & 10.31 & 13.7 & 6205.9 & $6190 \pm 40$ \\
\hline $170813 \mathrm{~A}$ & (L) & -0.51 & 111.36 & 111.87 & $\ldots$ & $\ldots$ & $\ldots$ & 9.5 & 265.3 & $260 \pm 70$ \\
\hline $170825 \mathrm{~B}$ & (L) & -0.51 & 6.14 & 6.66 & $\ldots$ & $\ldots$ & $\ldots$ & 0.9 & 1.5 & $0.6 \pm 0.3$ \\
\hline $170906 \mathrm{~A}$ & (L) & 12 & 90.88 & 78.85 & $\ldots$ & $\ldots$ & $\ldots$ & 162.1 & 1983.2 & $1821 \pm 3$ \\
\hline 170921B & (L) & 1.0 & 40.38 & 39.36 & $\ldots$ & $\ldots$ & $\ldots$ & 901.7 & 1058.6 & $200 \pm 200$ \\
\hline $171010 \mathrm{~A}$ & (L) & 17 & 123.91 & 107.27 & $\ldots$ & $\ldots$ & $\ldots$ & 335.6 & 2984.8 & $2650 \pm 50$ \\
\hline $171011 \mathrm{C}$ & (S) & -0.45 & 0.03 & 0.48 & $\ldots$ & $\ldots$ & $\ldots$ & 0.1 & 42.7 & $40 \pm 20$ \\
\hline $171102 \mathrm{~A}$ & (L) & 7.7 & 56.06 & 48.38 & $\ldots$ & $\ldots$ & $\ldots$ & 34.9 & 401.0 & $366 \pm 10$ \\
\hline $171120 \mathrm{~A}$ & (L) & 0.003 & 44.06 & 44.06 & $\ldots$ & $\ldots$ & $\ldots$ & 0.3 & 5276.0 & $5280 \pm 10$ \\
\hline $171124 \mathrm{~A}$ & (L) & -0.70 & 25.47 & 26.18 & $\ldots$ & $\ldots$ & $\ldots$ & 3.4 & 321.3 & $318 \pm 7$ \\
\hline $171210 \mathrm{~A}$ & (L) & 3.6 & 146.69 & 143.11 & -1.19 & 7.01 & 8.20 & 5.9 & 1518.1 & $1510 \pm 70$ \\
\hline $171212 \mathrm{~B}$ & (L) & -1.0 & 30.98 & 32.00 & $\ldots$ & $\ldots$ & $\ldots$ & 116.6 & 497.0 & $400 \pm 100$ \\
\hline $180113 \mathrm{C}$ & (L) & 5.4 & 29.95 & 24.58 & 5.85 & 29.54 & 23.69 & $\ldots$ & $\ldots$ & $\ldots$ \\
\hline $180210 \mathrm{~A}$ & (L) & 4.1 & 43.01 & 38.91 & $\ldots$ & $\ldots$ & $\ldots$ & 23.1 & 1621.1 & $1600 \pm 200$ \\
\hline $180305 \mathrm{~A}$ & (L) & 1.5 & 14.59 & 13.06 & 2.48 & 5.38 & 2.89 & 1613.8 & 2038.0 & $400 \pm 400$ \\
\hline $180526 \mathrm{~A}$ & (L) & * & 87.00 & 87.00 & $\ldots$ & $\ldots$ & $\ldots$ & 808.9 & 1948.6 & $1140 \pm 50$ \\
\hline $180703 \mathrm{~A}$ & (L) & 1.5 & 22.27 & 20.74 & 0.93 & 8.05 & 7.12 & 3.8 & 1614.0 & $1610 \pm 80$ \\
\hline 180703B & (S) & 0.13 & 1.66 & 1.54 & $\ldots$ & $\ldots$ & $\ldots$ & 8.0 & 78.5 & $70 \pm 20$ \\
\hline 180718B & (L) & 1.6 & 99.91 & 98.31 & $\ldots$ & $\ldots$ & $\ldots$ & 1.1 & 16.2 & $15 \pm 3$ \\
\hline 180720B & (L) & 4.4 & 53.25 & 48.90 & 0.91 & 31.47 & 30.55 & 11.8 & 625.0 & $613 \pm 9$ \\
\hline
\end{tabular}


Table 4

Likelihood Analysis

\begin{tabular}{|c|c|c|c|c|c|c|c|c|}
\hline GRB Name & $\begin{array}{l}\text { Interval }\left(t_{0}-t_{1}\right) \\
\quad(\mathrm{s})\end{array}$ & $\begin{array}{l}\text { Trans. Ev. } \\
\text { in the ROI }\end{array}$ & $\begin{array}{l}\text { Trans. Ev. } \\
\text { Predicted }\end{array}$ & $\begin{array}{c}\text { Test } \\
\text { Statistic } \\
(\mathrm{TS})\end{array}$ & Spectral Index & $\begin{array}{c}\text { Flux } \\
\left(\times 10^{-5} \mathrm{~cm}^{-2} \mathrm{~s}^{-1}\right)\end{array}$ & $\begin{array}{c}\text { Fluence } \\
\left(\times 10^{-5} \mathrm{erg} \mathrm{cm}^{-2}\right)\end{array}$ & $\begin{array}{c}E_{\text {iso }}(100 \mathrm{MeV}- \\
10 \mathrm{GeV}) \\
\left(\times 10^{52} \mathrm{erg}\right)\end{array}$ \\
\hline 080818B & LTF $(0.0-10000.0)$ & 65 & 5.0 & 23 & $-1.8 \pm 0.4$ & $0.08 \pm 0.05$ & $2 \pm 2$ & $\cdots$ \\
\hline \multirow[t]{4}{*}{$080825 \mathrm{C}$} & GBM (1.2-22.2) & 11 & 9.6 & 51 & $-3.8 \pm 0.8$ & $21 \pm 7$ & $0.11 \pm 0.04$ & $\cdots$ \\
\hline & LTF $(0.0-100.0)$ & 23 & 18.8 & 87 & $-3.0 \pm 0.4$ & $8 \pm 2$ & $0.26 \pm 0.08$ & $\ldots$ \\
\hline & EXT (22.2-173.5) & 14 & 10.1 & 23 & $-2.4 \pm 0.4$ & $3 \pm 1$ & $0.2 \pm 0.1$ & $\ldots$ \\
\hline & LAT (3.1-173.5) & 25 & 19.8 & 86 & $-2.8 \pm 0.4$ & $5 \pm 1$ & $0.29 \pm 0.10$ & $\cdots$ \\
\hline \multirow[t]{4}{*}{ 080916C } & GBM (1.3-64.3) & 255 & 246.8 & 2344 & $-2.22 \pm 0.07$ & $91 \pm 6$ & $4.0 \pm 0.6$ & $170 \pm 10$ \\
\hline & LTF $(0.0-100.0)$ & 290 & 277.7 & 2546 & $-2.21 \pm 0.07$ & $64 \pm 4$ & $4.5 \pm 0.7$ & $190 \pm 10$ \\
\hline & EXT (64.3-1531.8) & 186 & 106.0 & 352 & $-2.2 \pm 0.1$ & $1.6 \pm 0.2$ & $1.8 \pm 0.4$ & $68 \pm 9$ \\
\hline & LAT (3.0-1531.8) & 440 & 349.2 & 2173 & $-2.20 \pm 0.06$ & $5.1 \pm 0.3$ & $5.6 \pm 0.8$ & $230 \pm 10$ \\
\hline \multirow[t]{4}{*}{081006} & GBM $(-0.3-6.1)$ & 8 & 8.0 & 97 & $-2.2 \pm 0.4$ & $21 \pm 7$ & $0.10 \pm 0.07$ & $\ldots$ \\
\hline & LTF $(0.0-10000.0)$ & 46 & 15.9 & 74 & $-2.1 \pm 0.3$ & $1.6 \pm 0.5$ & $14 \pm 8$ & $\cdots$ \\
\hline & EXT (6.1-62.3) & 9 & 5.6 & 36 & $-1.9 \pm 0.4$ & $1.6 \pm 0.7$ & $0.1 \pm 0.1$ & $\cdots$ \\
\hline & LAT (0.8-62.3) & 16 & 12.7 & 112 & $-2.0 \pm 0.3$ & $3.3 \pm 1.0$ & $0.2 \pm 0.1$ & $\cdots$ \\
\hline \multirow[t]{3}{*}{081009} & LTF (0.0-4000.0) & 67 & 14.6 & 49 & $-1.7 \pm 0.2$ & $0.20 \pm 0.07$ & $2 \pm 1$ & $\cdots$ \\
\hline & EXT (42.7-1250.1) & 45 & 14.7 & 49 & $-1.8 \pm 0.2$ & $0.3 \pm 0.1$ & $0.8 \pm 0.5$ & $\cdots$ \\
\hline & LAT (67.8-1250.1) & 44 & 13.6 & 46 & $-1.8 \pm 0.2$ & $0.3 \pm 0.1$ & $0.8 \pm 0.5$ & $\cdots$ \\
\hline \multirow[t]{4}{*}{ 081024B } & GBM $(-0.1-0.6)$ & 6 & 6.0 & 76 & $-2.3 \pm 0.5$ & $140 \pm 60$ & $0.06 \pm 0.05$ & $\cdots$ \\
\hline & LTF $(0.0-10.0)$ & 14 & 13.9 & 152 & $-2.3 \pm 0.3$ & $21 \pm 6$ & $0.13 \pm 0.07$ & $\ldots$ \\
\hline & EXT (0.6-2.2) & 6 & 6.0 & 75 & $-2.3 \pm 0.5$ & $60 \pm 20$ & $0.05 \pm 0.04$ & $\ldots$ \\
\hline & LAT (0.2-2.2) & 11 & 11.0 & 138 & $-2.2 \pm 0.4$ & $80 \pm 30$ & $0.11 \pm 0.08$ & $\cdots$ \\
\hline \multirow[t]{3}{*}{ 081102B } & GBM $(-0.1-1.7)$ & 6 & 6.0 & 70 & $-2.2 \pm 0.5$ & $80 \pm 30$ & $0.10 \pm 0.09$ & $\cdots$ \\
\hline & LTF $(0.0-10.0)$ & 6 & 5.8 & 50 & $-2.2 \pm 0.5$ & $14 \pm 6$ & $0.09 \pm 0.09$ & $\cdots$ \\
\hline & LAT $(0.3-0.4)$ & 5 & 5.0 & 88 & $-2.5 \pm 0.6$ & $800 \pm 400$ & $0.06 \pm 0.04$ & $\cdots$ \\
\hline 081122A & LTF $(0.0-100.0)$ & 6 & 5.4 & 37 & $-1.8 \pm 0.4$ & $0.8 \pm 0.3$ & $0.2 \pm 0.2$ & $\cdots$ \\
\hline \multirow[t]{2}{*}{ 081203A } & LTF $(0.0-500.0)$ & 7 & 6.0 & 34 & $-2.1 \pm 0.4$ & $0.3 \pm 0.1$ & $0.10 \pm 0.10$ & $1.0 \pm 0.4$ \\
\hline & EXT (214.0-348.1) & 4 & 3.9 & 22 & $-2.4 \pm 0.6$ & $0.5 \pm 0.3$ & $0.04 \pm 0.04$ & $0.6 \pm 0.3$ \\
\hline 081224 & LTF $(0.0-500.0)$ & 21 & 6.1 & 27 & $-1.8 \pm 0.3$ & $0.19 \pm 0.09$ & $0.2 \pm 0.2$ & $\cdots$ \\
\hline \multirow[t]{3}{*}{090217} & GBM (0.8-34.1) & 22 & 21.1 & 177 & $-2.3 \pm 0.3$ & $11 \pm 2$ & $0.21 \pm 0.09$ & $\cdots$ \\
\hline & LTF $(0.0-100.0)$ & 30 & 25.5 & 162 & $-2.3 \pm 0.2$ & $4.5 \pm 1.0$ & $0.3 \pm 0.1$ & $\ldots$ \\
\hline & LAT (4.1-71.6) & 27 & 23.6 & 156 & $-2.3 \pm 0.3$ & $6 \pm 1$ & $0.2 \pm 0.1$ & $\cdots$ \\
\hline \multirow[t]{2}{*}{ 090227A } & LTF $(0.0-500.0)$ & 16 & 7.7 & 33 & $-2.0 \pm 0.4$ & $0.3 \pm 0.1$ & $0.1 \pm 0.1$ & $\cdots$ \\
\hline & LAT (2.9-204.3) & 5 & 3.8 & 30 & $-1.7 \pm 0.4$ & $0.3 \pm 0.2$ & $0.1 \pm 0.2$ & $\cdots$ \\
\hline \multirow[t]{2}{*}{ 090227B } & GBM $(-0.0-0.3)$ & 2 & 2.0 & 23 & $-6.00 \pm 0.01$ & $2000 \pm 1000$ & $0.10 \pm 0.07$ & $\cdots$ \\
\hline & $\operatorname{LTF}(0.0-1.2)$ & 3 & 3.0 & 30 & $-5 \pm 2$ & $600 \pm 300$ & $0.15 \pm 0.10$ & $\cdots$ \\
\hline \multirow{3}{*}{ 090228A } & $\operatorname{LTF}(0.0-10.0)$ & 6 & 5.8 & 60 & $-1.8 \pm 0.3$ & $9 \pm 4$ & $0.2 \pm 0.2$ & $\cdots$ \\
\hline & EXT (0.4-2.1) & 4 & 3.9 & 39 & $-1.9 \pm 0.5$ & $40 \pm 20$ & $0.1 \pm 0.1$ & $\cdots$ \\
\hline & LAT $(0.1-2.1)$ & 4 & 4.0 & 38 & $-1.9 \pm 0.5$ & $30 \pm 20$ & $0.1 \pm 0.1$ & $\cdots$ \\
\hline \multirow[t]{4}{*}{090323} & GBM (8.7-142.6) & 23 & 19.2 & 89 & $-3.0 \pm 0.4$ & $6 \pm 2$ & $0.26 \pm 0.07$ & $30 \pm 20$ \\
\hline & LTF $(0.0-4000.0)$ & 73 & 63.8 & 392 & $-2.4 \pm 0.2$ & $2.8 \pm 0.4$ & $6 \pm 2$ & $260 \pm 50$ \\
\hline & $\begin{array}{r}\text { EXT } \\
(142.6-5321.6)\end{array}$ & 80 & 49.8 & 169 & $-2.2 \pm 0.2$ & $0.8 \pm 0.1$ & $3 \pm 1$ & $90 \pm 20$ \\
\hline & LAT (9.3-5321.6) & 102 & 67.2 & 206 & $-2.3 \pm 0.2$ & $1.0 \pm 0.1$ & $3.4 \pm 0.9$ & $120 \pm 20$ \\
\hline \multirow[t]{4}{*}{090328} & GBM (4.4-66.0) & 12 & 11.8 & 102 & $-3.0 \pm 0.5$ & $14 \pm 4$ & $0.27 \pm 0.09$ & $0.7 \pm 0.2$ \\
\hline & LTF (0.0-4000.0) & 170 & 87.8 & 288 & $-2.2 \pm 0.1$ & $0.62 \pm 0.08$ & $1.7 \pm 0.4$ & $2.3 \pm 0.3$ \\
\hline & EXT (66.0-6150.6) & 188 & 78.7 & 255 & $-2.2 \pm 0.1$ & $0.42 \pm 0.06$ & $1.9 \pm 0.6$ & $2.4 \pm 0.5$ \\
\hline & LAT (14.4-6150.6) & 199 & 86.5 & 275 & $-2.2 \pm 0.1$ & $0.46 \pm 0.06$ & $2.0 \pm 0.5$ & $2.7 \pm 0.4$ \\
\hline \multirow[t]{2}{*}{ 090427A } & LTF $(0.0-500.0)$ & 10 & 3.7 & 40 & $-1.3 \pm 0.3$ & $0.5 \pm 0.3$ & $2 \pm 2$ & $\cdots$ \\
\hline & EXT (15.0-435.6) & 5 & 1.1 & 24 & $-0.8 \pm 0.7$ & $0.2 \pm 0.2$ & $4 \pm 5$ & $\cdots$ \\
\hline \multirow[t]{4}{*}{090510} & GBM $(-0.0-0.9)$ & 64 & 64.0 & 1285 & $-1.8 \pm 0.1$ & $900 \pm 100$ & $1.7 \pm 0.6$ & $1.7 \pm 0.3$ \\
\hline & LTF (0.0-10.0) & 221 & 220.8 & 3618 & $-1.99 \pm 0.07$ & $310 \pm 20$ & $3.5 \pm 0.6$ & $5.1 \pm 0.4$ \\
\hline & EXT (0.9-170.0) & 205 & 197.8 & 1869 & $-2.15 \pm 0.08$ & $17 \pm 1$ & $2.2 \pm 0.4$ & $4.2 \pm 0.4$ \\
\hline & LAT $(0.0-170.0)$ & 267 & 262.2 & 2924 & $-2.05 \pm 0.06$ & $22 \pm 1$ & $3.6 \pm 0.6$ & $5.8 \pm 0.5$ \\
\hline
\end{tabular}


Table 4

(Continued)

\begin{tabular}{|c|c|c|c|c|c|c|c|c|}
\hline GRB Name & $\begin{array}{l}\text { Interval }\left(t_{0}-t_{1}\right) \\
\quad(\mathrm{s})\end{array}$ & $\begin{array}{l}\text { Trans. Ev. } \\
\text { in the ROI }\end{array}$ & $\begin{array}{l}\text { Trans. Ev. } \\
\text { Predicted }\end{array}$ & $\begin{array}{c}\text { Test } \\
\text { Statistic } \\
\text { (TS) }\end{array}$ & Spectral Index & $\begin{array}{c}\text { Flux } \\
\left(\times 10^{-5} \mathrm{~cm}^{-2} \mathrm{~s}^{-1}\right)\end{array}$ & $\begin{array}{c}\text { Fluence } \\
\left(\times 10^{-5} \mathrm{erg} \mathrm{cm}^{-2}\right)\end{array}$ & $\begin{array}{c}E_{\text {iso }}(100 \mathrm{MeV}- \\
10 \mathrm{GeV}) \\
\left(\times 10^{52} \mathrm{erg}\right)\end{array}$ \\
\hline \multirow[t]{3}{*}{090626} & LTF (0.0-4000.0) & 51 & 31.2 & 120 & $-2.1 \pm 0.2$ & $1.0 \pm 0.2$ & $4 \pm 1$ & $\cdots$ \\
\hline & EXT (50.4-557.3) & 38 & 23.5 & 102 & $-2.0 \pm 0.2$ & $1.1 \pm 0.3$ & $0.6 \pm 0.3$ & $\ldots$ \\
\hline & LAT (9.0-557.3) & 41 & 25.1 & 101 & $-2.1 \pm 0.2$ & $1.1 \pm 0.3$ & $0.6 \pm 0.3$ & $\cdots$ \\
\hline \multirow[t]{3}{*}{ 090720B } & GBM $(-0.3-10.5)$ & 7 & 6.5 & 50 & $-2.5 \pm 0.6$ & $20 \pm 8$ & $0.10 \pm 0.07$ & $\ldots$ \\
\hline & LTF $(0.0-10.0)$ & 7 & 6.5 & 51 & $-2.5 \pm 0.6$ & $21 \pm 9$ & $0.10 \pm 0.07$ & $\ldots$ \\
\hline & LAT (0.1-1.7) & 5 & 4.9 & 51 & $-2.3 \pm 0.5$ & $100 \pm 50$ & $0.1 \pm 0.1$ & $\ldots$ \\
\hline \multirow{4}{*}{ 090902B } & GBM (2.8-22.1) & 245 & 244.6 & 3795 & $-1.99 \pm 0.06$ & $310 \pm 20$ & $7 \pm 1$ & $37 \pm 3$ \\
\hline & LTF (0.0-100.0) & 392 & 389.1 & 5006 & $-1.94 \pm 0.05$ & $84 \pm 4$ & $11 \pm 2$ & $54 \pm 3$ \\
\hline & EXT (22.1-884.2) & 298 & 246.0 & 1723 & $-1.92 \pm 0.06$ & $4.1 \pm 0.3$ & $4.9 \pm 0.9$ & $23 \pm 2$ \\
\hline & LAT (0.5-884.2) & 548 & 500.5 & 4089 & $-1.94 \pm 0.04$ & $8.3 \pm 0.4$ & $9 \pm 1$ & $47 \pm 2$ \\
\hline \multirow[t]{4}{*}{ 090926A } & GBM (2.2-15.9) & 252 & 251.9 & 3806 & $-2.52 \pm 0.09$ & $450 \pm 30$ & $2.9 \pm 0.3$ & $53 \pm 4$ \\
\hline & LTF (0.0-100.0) & 386 & 383.3 & 4509 & $-2.26 \pm 0.06$ & $95 \pm 5$ & $6.2 \pm 0.7$ & $76 \pm 4$ \\
\hline & EXT (15.9-4419.5) & 225 & 166.9 & 707 & $-1.86 \pm 0.07$ & $1.6 \pm 0.1$ & $12 \pm 2$ & $60 \pm 5$ \\
\hline & LAT (2.2-4419.5) & 477 & 417.0 & 2652 & $-2.14 \pm 0.05$ & $4.2 \pm 0.2$ & $15 \pm 2$ & $149 \pm 8$ \\
\hline \multirow[t]{4}{*}{091003} & GBM $(0.8-21.1)$ & 6 & 5.2 & 63 & $-1.8 \pm 0.4$ & $4 \pm 2$ & $0.2 \pm 0.2$ & $0.16 \pm 0.08$ \\
\hline & LTF $(0.0-500.0)$ & 47 & 29.4 & 171 & $-1.8 \pm 0.2$ & $0.9 \pm 0.2$ & $0.8 \pm 0.4$ & $0.9 \pm 0.2$ \\
\hline & EXT (21.1-392.0) & 37 & 23.8 & 134 & $-1.8 \pm 0.2$ & $1.0 \pm 0.2$ & $0.7 \pm 0.4$ & $0.7 \pm 0.2$ \\
\hline & LAT (1.0-392.0) & 42 & 28.0 & 174 & $-1.8 \pm 0.2$ & $1.2 \pm 0.2$ & $0.8 \pm 0.4$ & $0.8 \pm 0.2$ \\
\hline \multirow[t]{4}{*}{091031} & GBM (1.4-35.3) & 13 & 10.7 & 67 & $-2.7 \pm 0.5$ & $5 \pm 2$ & $0.07 \pm 0.03$ & $\ldots$ \\
\hline & LTF (0.0-100.0) & 30 & 24.5 & 166 & $-2.2 \pm 0.2$ & $4.0 \pm 0.9$ & $0.3 \pm 0.1$ & $\cdots$ \\
\hline & EXT (35.3-408.2) & 30 & 14.4 & 80 & $-1.8 \pm 0.2$ & $0.6 \pm 0.2$ & $0.4 \pm 0.3$ & $\cdots$ \\
\hline & LAT (0.1-408.2) & 47 & 26.0 & 110 & $-2.1 \pm 0.2$ & $1.0 \pm 0.2$ & $0.4 \pm 0.2$ & $\cdots$ \\
\hline \multirow[t]{3}{*}{091120} & LTF (0.0-4000.0) & 67 & 22.0 & 45 & $-2.8 \pm 0.4$ & $0.4 \pm 0.1$ & $0.5 \pm 0.2$ & $\ldots$ \\
\hline & EXT (51.2-803.9) & 40 & 16.9 & 37 & $-2.7 \pm 0.4$ & $0.5 \pm 0.1$ & $0.13 \pm 0.05$ & $\cdots$ \\
\hline & LAT (31.8-803.9) & 41 & 17.6 & 37 & $-2.8 \pm 0.4$ & $0.5 \pm 0.2$ & $0.14 \pm 0.05$ & $\cdots$ \\
\hline \multirow[t]{2}{*}{091127} & LTF $(0.0-500.0)$ & 14 & 6.2 & 34 & $-1.7 \pm 0.3$ & $0.19 \pm 0.09$ & $0.3 \pm 0.3$ & $0.06 \pm 0.03$ \\
\hline & LAT (8.6-35.4) & 3 & 2.1 & 32 & $-1.2 \pm 0.4$ & $1.2 \pm 0.9$ & $0.4 \pm 0.5$ & $0.03 \pm 0.02$ \\
\hline \multirow[t]{4}{*}{$100116 \mathrm{~A}$} & GBM (0.6-103.1) & 12 & 8.5 & 28 & $-3.2 \pm 0.7$ & $1.7 \pm 0.6$ & $0.05 \pm 0.02$ & $\cdots$ \\
\hline & LTF (0.0-4000.0) & 67 & 25.7 & 129 & $-1.8 \pm 0.2$ & $0.7 \pm 0.2$ & $5 \pm 2$ & $\cdots$ \\
\hline & EXT (103.1-730.4) & 52 & 16.5 & 103 & $-1.6 \pm 0.2$ & $0.5 \pm 0.2$ & $1.3 \pm 0.7$ & $\cdots$ \\
\hline & LAT (77.5-730.4) & 60 & 23.6 & 122 & $-1.8 \pm 0.2$ & $0.8 \pm 0.2$ & $1.0 \pm 0.5$ & $\cdots$ \\
\hline \multirow[t]{3}{*}{$100213 C$} & LTF $(0.0-4000.0)$ & 181 & 9.8 & 42 & $-1.7 \pm 0.3$ & $0.16 \pm 0.08$ & $2 \pm 1$ & $\cdots$ \\
\hline & EXT (60.0-3215.3) & 82 & 7.4 & 22 & $-2.0 \pm 0.4$ & $0.3 \pm 0.2$ & $1.0 \pm 0.8$ & $\cdots$ \\
\hline & $\begin{array}{r}\text { LAT } \\
(2707.2-3215.3)\end{array}$ & 59 & 5.6 & 21 & $-1.9 \pm 0.4$ & $0.3 \pm 0.2$ & $0.3 \pm 0.3$ & $\cdots$ \\
\hline \multirow[t]{4}{*}{$100225 \mathrm{~A}$} & GBM $(-0.3-12.7)$ & 7 & 6.8 & 47 & $-3.7 \pm 1.0$ & $18 \pm 7$ & $0.06 \pm 0.02$ & $\cdots$ \\
\hline & LTF $(0.0-100.0)$ & 13 & 10.1 & 46 & $-2.3 \pm 0.4$ & $3 \pm 1$ & $0.2 \pm 0.1$ & $\cdots$ \\
\hline & EXT (12.7-1012.4) & 36 & 7.2 & 27 & $-1.8 \pm 0.4$ & $0.13 \pm 0.07$ & $0.2 \pm 0.2$ & $\ldots$ \\
\hline & LAT (6.6-1012.4) & 43 & 12.1 & 32 & $-2.2 \pm 0.4$ & $0.22 \pm 0.09$ & $0.2 \pm 0.1$ & $\cdots$ \\
\hline \multirow[t]{3}{*}{$100325 \mathrm{~A}$} & GBM (-0.4-6.7) & 6 & 6.0 & 58 & $-2.1 \pm 0.4$ & $12 \pm 5$ & $0.07 \pm 0.07$ & $\cdots$ \\
\hline & $\operatorname{LTF}(0.0-10.0)$ & 6 & 5.9 & 55 & $-2.1 \pm 0.4$ & $8 \pm 4$ & $0.07 \pm 0.07$ & $\ldots$ \\
\hline & LAT $(0.2-1.2)$ & 5 & 5.0 & 68 & $-1.9 \pm 0.4$ & $70 \pm 30$ & $0.1 \pm 0.1$ & $\cdots$ \\
\hline \multirow[t]{4}{*}{$100414 \mathrm{~A}$} & GBM (1.9-28.4) & 5 & 4.7 & 35 & $-2.3 \pm 0.5$ & $20 \pm 10$ & $0.4 \pm 0.3$ & $2.1 \pm 1.0$ \\
\hline & LTF (0.0-4000.0) & 97 & 48.8 & 211 & $-1.8 \pm 0.1$ & $0.43 \pm 0.07$ & $3 \pm 1$ & $7 \pm 1$ \\
\hline & EXT (28.4-5506.1) & 121 & 50.2 & 210 & $-1.8 \pm 0.1$ & $0.30 \pm 0.05$ & $3 \pm 1$ & $7 \pm 1$ \\
\hline & LAT (18.7-5506.1) & 125 & 52.6 & 216 & $-1.8 \pm 0.1$ & $0.31 \pm 0.05$ & $3 \pm 1$ & $7 \pm 1$ \\
\hline \multirow[t]{3}{*}{ 100423B } & LTF (0.0-500.0) & 19 & 9.0 & 31 & $-2.0 \pm 0.4$ & $0.4 \pm 0.2$ & $0.2 \pm 0.2$ & $\ldots$ \\
\hline & EXT (18.1-180.6) & 11 & 5.8 & 28 & $-1.9 \pm 0.5$ & $0.8 \pm 0.5$ & $0.2 \pm 0.2$ & $\cdots$ \\
\hline & LAT (166.7-180.6) & 2 & 2.0 & 23 & $-1.8 \pm 0.6$ & $3 \pm 2$ & $0.1 \pm 0.1$ & $\cdots$ \\
\hline \multirow[t]{3}{*}{$100511 \mathrm{~A}$} & LTF $(0.0-500.0)$ & 44 & 18.5 & 114 & $-1.5 \pm 0.2$ & $0.6 \pm 0.2$ & $1.5 \pm 1.0$ & $\ldots$ \\
\hline & EXT (43.3-6338.2) & 175 & 30.4 & 89 & $-1.8 \pm 0.2$ & $0.18 \pm 0.05$ & $2 \pm 1$ & $\cdots$ \\
\hline & LAT (11.6-6338.2) & 178 & 33.0 & 93 & $-1.8 \pm 0.2$ & $0.19 \pm 0.05$ & $2 \pm 1$ & $\ldots$ \\
\hline $100620 \mathrm{~A}$ & GBM $(0.2-52.0)$ & 6 & 5.8 & 28 & $-4 \pm 1$ & $1.8 \pm 0.8$ & $0.02 \pm 0.01$ & $\cdots$ \\
\hline
\end{tabular}


Table 4

(Continued)

\begin{tabular}{|c|c|c|c|c|c|c|c|c|}
\hline GRB Name & $\begin{array}{l}\text { Interval }\left(t_{0}-t_{1}\right) \\
\quad(\mathrm{s})\end{array}$ & $\begin{array}{l}\text { Trans. Ev. } \\
\text { in the ROI }\end{array}$ & $\begin{array}{l}\text { Trans. Ev. } \\
\text { Predicted }\end{array}$ & $\begin{array}{c}\text { Test } \\
\text { Statistic } \\
\text { (TS) }\end{array}$ & Spectral Index & $\begin{array}{c}\text { Flux } \\
\left(\times 10^{-5} \mathrm{~cm}^{-2} \mathrm{~s}^{-1}\right)\end{array}$ & $\begin{array}{c}\text { Fluence } \\
\left(\times 10^{-5} \mathrm{erg} \mathrm{cm}^{-2}\right)\end{array}$ & $\begin{array}{c}E_{\text {iso }}(100 \mathrm{MeV}- \\
10 \mathrm{GeV}) \\
\left(\times 10^{52} \mathrm{erg}\right)\end{array}$ \\
\hline & LTF $(0.0-100.0)$ & 8 & 6.4 & 26 & $-3.5 \pm 0.9$ & $1.0 \pm 0.4$ & $0.03 \pm 0.01$ & $\ldots$ \\
\hline & LAT $(3.8-42.8)$ & 4 & 3.8 & 21 & $-4 \pm 2$ & $1.6 \pm 0.9$ & $0.015 \pm 0.009$ & $\cdots$ \\
\hline \multirow[t]{3}{*}{ 100724B } & GBM (8.2-122.9) & 9 & 8.5 & 53 & $-5 \pm 1$ & $7 \pm 3$ & $0.19 \pm 0.07$ & $\ldots$ \\
\hline & LTF $(0.0-4000.0)$ & 35 & 17.0 & 23 & $-3.7 \pm 0.6$ & $0.22 \pm 0.07$ & $0.22 \pm 0.07$ & $\ldots$ \\
\hline & LAT (9.1-53.6) & 9 & 8.7 & 65 & $-4 \pm 1$ & $8 \pm 3$ & $0.08 \pm 0.03$ & $\cdots$ \\
\hline \multirow[t]{3}{*}{$100728 \mathrm{~A}$} & LTF $(0.0-4000.0)$ & 107 & 16.1 & 64 & $-1.7 \pm 0.2$ & $0.13 \pm 0.04$ & $1.3 \pm 0.8$ & $2.8 \pm 0.8$ \\
\hline & $\begin{array}{r}\text { EXT } \\
(178.7-1340.5)\end{array}$ & 62 & 12.8 & 64 & $-1.7 \pm 0.2$ & $0.17 \pm 0.06$ & $0.6 \pm 0.4$ & $1.1 \pm 0.4$ \\
\hline & $\begin{array}{r}\text { LAT } \\
(248.6-1340.5)\end{array}$ & 60 & 10.7 & 54 & $-1.6 \pm 0.2$ & $0.15 \pm 0.06$ & $0.5 \pm 0.4$ & $0.9 \pm 0.3$ \\
\hline \multirow[t]{3}{*}{$100826 \mathrm{~A}$} & GBM (8.7-93.7) & 7 & 5.8 & 34 & $-1.6 \pm 0.4$ & $14 \pm 7$ & $4 \pm 5$ & $\cdots$ \\
\hline & LTF $(0.0-100.0)$ & 7 & 5.7 & 32 & $-1.6 \pm 0.4$ & $11 \pm 6$ & $4 \pm 5$ & $\cdots$ \\
\hline & LAT (33.5-54.5) & 4 & 3.8 & 23 & $-2.3 \pm 0.6$ & $40 \pm 20$ & $0.5 \pm 0.4$ & $\cdots$ \\
\hline \multirow[t]{3}{*}{$101014 \mathrm{~A}$} & LTF $(0.0-10000.0)$ & 103 & 6.8 & 48 & $-1.4 \pm 0.3$ & $0.019 \pm 0.010$ & $2 \pm 1$ & $\cdots$ \\
\hline & $\begin{array}{r}\text { EXT } \\
(450.8-4196.0)\end{array}$ & 42 & 5.3 & 46 & $-1.3 \pm 0.3$ & $0.04 \pm 0.02$ & $1 \pm 1$ & $\cdots$ \\
\hline & $\begin{array}{r}\text { LAT } \\
(2270.7-4196.0)\end{array}$ & 40 & 5.2 & 47 & $-1.3 \pm 0.3$ & $0.04 \pm 0.02$ & $0.7 \pm 0.8$ & $\cdots$ \\
\hline \multirow[t]{3}{*}{ 101107A } & GBM $(2.3-378.1)$ & 13 & 8.2 & 49 & $-1.9 \pm 0.3$ & $0.4 \pm 0.2$ & $0.2 \pm 0.2$ & $\cdots$ \\
\hline & LTF $(0.0-500.0)$ & 17 & 10.6 & 55 & $-2.1 \pm 0.3$ & $0.4 \pm 0.1$ & $0.2 \pm 0.1$ & $\cdots$ \\
\hline & LAT (134.0-239.4) & 9 & 8.2 & 56 & $-1.9 \pm 0.3$ & $1.4 \pm 0.6$ & $0.2 \pm 0.2$ & $\cdots$ \\
\hline \multirow[t]{3}{*}{ 101227B } & GBM (0.8-154.1) & 14 & 5.0 & 24 & $-1.6 \pm 0.3$ & $0.4 \pm 0.2$ & $0.2 \pm 0.3$ & $\cdots$ \\
\hline & LTF (0.0-100.0) & 9 & 4.2 & 27 & $-1.5 \pm 0.3$ & $0.6 \pm 0.3$ & $0.3 \pm 0.3$ & $\cdots$ \\
\hline & LAT (17.3-23.8) & 3 & 2.1 & 25 & $-1.5 \pm 0.5$ & $4 \pm 3$ & $0.1 \pm 0.2$ & $\cdots$ \\
\hline \multirow[t]{3}{*}{$110120 \mathrm{~A}$} & LTF (0.0-4000.0) & 62 & 19.2 & 70 & $-2.0 \pm 0.2$ & $0.18 \pm 0.05$ & $0.7 \pm 0.4$ & $\cdots$ \\
\hline & EXT (26.2-1112.8) & 28 & 14.3 & 63 & $-2.0 \pm 0.3$ & $0.21 \pm 0.06$ & $0.3 \pm 0.2$ & $\cdots$ \\
\hline & LAT (0.6-1112.8) & 31 & 16.8 & 74 & $-2.0 \pm 0.3$ & $0.24 \pm 0.07$ & $0.3 \pm 0.2$ & $\cdots$ \\
\hline \multirow[t]{3}{*}{$110123 \mathrm{~A}$} & LTF $(0.0-10000.0)$ & 190 & 20.7 & 46 & $-2.1 \pm 0.3$ & $0.06 \pm 0.02$ & $0.5 \pm 0.3$ & $\cdots$ \\
\hline & EXT (18.6-526.2) & 28 & 11.3 & 38 & $-2.3 \pm 0.4$ & $0.4 \pm 0.1$ & $0.12 \pm 0.07$ & $\ldots$ \\
\hline & LAT (51.6-526.2) & 23 & 7.9 & 27 & $-2.2 \pm 0.4$ & $0.3 \pm 0.1$ & $0.10 \pm 0.08$ & $\cdots$ \\
\hline \multirow[t]{2}{*}{$110213 \mathrm{~A}$} & LTF (0.0-10000.0) & 207 & 8.8 & 28 & $-1.6 \pm 0.3$ & $0.05 \pm 0.03$ & $2 \pm 2$ & $3 \pm 1$ \\
\hline & EXT (33.5-1944.2) & 37 & 3.3 & 21 & $-1.5 \pm 0.4$ & $0.11 \pm 0.07$ & $1 \pm 1$ & $1.1 \pm 0.7$ \\
\hline \multirow[t]{3}{*}{ 110328B } & LTF (0.0-10000.0) & 41 & 15.7 & 50 & $-2.2 \pm 0.3$ & $0.15 \pm 0.05$ & $1.1 \pm 0.6$ & $\cdots$ \\
\hline & EXT (86.5-737.2) & 17 & 11.1 & 55 & $-2.0 \pm 0.3$ & $0.3 \pm 0.1$ & $0.2 \pm 0.2$ & $\cdots$ \\
\hline & LAT (102.5-737.2) & 17 & 11.1 & 55 & $-2.0 \pm 0.3$ & $0.3 \pm 0.1$ & $0.2 \pm 0.2$ & $\cdots$ \\
\hline \multirow[t]{3}{*}{$110428 \mathrm{~A}$} & LTF $(0.0-500.0)$ & 71 & 22.4 & 63 & $-2.0 \pm 0.2$ & $0.7 \pm 0.2$ & $0.4 \pm 0.2$ & $\cdots$ \\
\hline & EXT (8.3-393.5) & 51 & 17.0 & 52 & $-1.9 \pm 0.3$ & $0.7 \pm 0.2$ & $0.4 \pm 0.2$ & $\cdots$ \\
\hline & LAT (7.3-393.5) & 52 & 18.1 & 55 & $-1.9 \pm 0.2$ & $0.8 \pm 0.2$ & $0.4 \pm 0.2$ & $\cdots$ \\
\hline \multirow[t]{4}{*}{$110518 \mathrm{~A}$} & LTF (0.0-4000.0) & 51 & 10.4 & 42 & $-1.9 \pm 0.3$ & $0.09 \pm 0.04$ & $0.6 \pm 0.5$ & $\cdots$ \\
\hline & EXT (35.0-2364.2) & 36 & 8.4 & 31 & $-1.9 \pm 0.4$ & $0.13 \pm 0.06$ & $0.4 \pm 0.4$ & $\cdots$ \\
\hline & LAT & 16 & 3.5 & 21 & $-1.8 \pm 0.5$ & $0.14 \pm 0.10$ & $0.1 \pm 0.1$ & $\cdots$ \\
\hline & $(1968.2-2364.2)$ & & & & & & & \\
\hline \multirow[t]{3}{*}{$110625 \mathrm{~A}$} & LTF $(0.0-4000.0)$ & 177 & 57.3 & 88 & $-3 \pm 3$ & $2 \pm 6$ & $3.9 \pm 0.8$ & $\cdots$ \\
\hline & EXT (30.7-577.2) & 94 & 39.2 & 73 & $-2.7 \pm 0.3$ & $3.1 \pm 0.6$ & $0.7 \pm 0.2$ & $\cdots$ \\
\hline & LAT (205.9-577.2) & 86 & 36.6 & 67 & $-2.7 \pm 0.3$ & $3.1 \pm 0.6$ & $0.4 \pm 0.1$ & $\cdots$ \\
\hline \multirow[t]{4}{*}{$110721 \mathrm{~A}$} & GBM (0.0-21.8) & 34 & 33.6 & 355 & $-2.6 \pm 0.3$ & $30 \pm 5$ & $0.28 \pm 0.09$ & $\cdots$ \\
\hline & $\operatorname{LTF}(0.0-10.0)$ & 30 & 29.8 & 349 & $-2.7 \pm 0.3$ & $60 \pm 10$ & $0.23 \pm 0.07$ & $\cdots$ \\
\hline & EXT (21.8-120.6) & 8 & 7.4 & 31 & $-2.1 \pm 0.4$ & $1.3 \pm 0.5$ & $0.10 \pm 0.09$ & $\cdots$ \\
\hline & LAT (0.0-120.6) & 41 & 40.2 & 387 & $-2.4 \pm 0.2$ & $5.8 \pm 0.9$ & $0.4 \pm 0.1$ & $\cdots$ \\
\hline \multirow[t]{3}{*}{$110728 \mathrm{~A}$} & LTF $(0.0-10.0)$ & 8 & 7.7 & 73 & $-1.8 \pm 0.3$ & $14 \pm 5$ & $0.2 \pm 0.2$ & $\cdots$ \\
\hline & EXT (0.6-3.0) & 5 & 4.9 & 39 & $-1.9 \pm 0.4$ & $40 \pm 20$ & $0.1 \pm 0.1$ & $\ldots$ \\
\hline & LAT (0.4-3.0) & 6 & 5.9 & 57 & $-1.9 \pm 0.4$ & $40 \pm 20$ & $0.2 \pm 0.2$ & $\cdots$ \\
\hline
\end{tabular}


Table 4

(Continued)

\begin{tabular}{|c|c|c|c|c|c|c|c|c|}
\hline GRB Name & $\begin{array}{l}\text { Interval }\left(t_{0}-t_{1}\right) \\
\quad(\mathrm{s})\end{array}$ & $\begin{array}{l}\text { Trans. Ev. } \\
\text { in the ROI }\end{array}$ & $\begin{array}{l}\text { Trans. Ev. } \\
\text { Predicted }\end{array}$ & $\begin{array}{c}\text { Test } \\
\text { Statistic } \\
\text { (TS) }\end{array}$ & Spectral Index & $\begin{array}{c}\text { Flux } \\
\left(\times 10^{-5} \mathrm{~cm}^{-2} \mathrm{~s}^{-1}\right)\end{array}$ & $\begin{array}{c}\text { Fluence } \\
\left(\times 10^{-5} \mathrm{erg} \mathrm{cm}^{-2}\right)\end{array}$ & $\begin{array}{c}E_{\text {iso }}(100 \mathrm{MeV}- \\
10 \mathrm{GeV}) \\
\left(\times 10^{52} \mathrm{erg}\right)\end{array}$ \\
\hline \multirow[t]{4}{*}{$110731 \mathrm{~A}$} & GBM (0.0-7.5) & 50 & 49.4 & 469 & $-2.6 \pm 0.2$ & $100 \pm 10$ & $0.30 \pm 0.07$ & $12 \pm 3$ \\
\hline & LTF $(0.0-10.0)$ & 59 & 58.2 & 558 & $-2.4 \pm 0.2$ & $80 \pm 10$ & $0.4 \pm 0.1$ & $13 \pm 2$ \\
\hline & EXT (7.5-436.0) & 111 & 28.4 & 89 & $-2.0 \pm 0.2$ & $0.9 \pm 0.2$ & $0.4 \pm 0.2$ & $5 \pm 1$ \\
\hline & LAT (1.1-436.0) & 160 & 70.6 & 191 & $-2.3 \pm 0.2$ & $2.4 \pm 0.3$ & $0.6 \pm 0.2$ & $15 \pm 2$ \\
\hline \multirow[t]{3}{*}{$110903 \mathrm{~A}$} & GBM $(-0.3-341.0)$ & 11 & 5.5 & 45 & $-1.5 \pm 0.3$ & $0.4 \pm 0.2$ & $0.6 \pm 0.6$ & $\cdots$ \\
\hline & LTF $(0.0-500.0)$ & 14 & 7.2 & 49 & $-1.6 \pm 0.3$ & $0.3 \pm 0.2$ & $0.6 \pm 0.5$ & $\cdots$ \\
\hline & LAT (46.7-370.1) & 12 & 5.6 & 48 & $-1.4 \pm 0.3$ & $0.4 \pm 0.2$ & $0.7 \pm 0.7$ & $\cdots$ \\
\hline \multirow[t]{4}{*}{ 110921B } & GBM (0.9-18.6) & 6 & 5.0 & 43 & $-2.0 \pm 0.4$ & $6 \pm 3$ & $0.1 \pm 0.1$ & $\ldots$ \\
\hline & LTF $(0.0-500.0)$ & 21 & 11.9 & 55 & $-1.9 \pm 0.3$ & $0.4 \pm 0.1$ & $0.3 \pm 0.2$ & $\cdots$ \\
\hline & EXT (18.6-321.6) & 14 & 7.4 & 36 & $-1.9 \pm 0.3$ & $0.4 \pm 0.2$ & $0.2 \pm 0.2$ & $\cdots$ \\
\hline & LAT (7.4-321.6) & 19 & 11.0 & 55 & $-1.8 \pm 0.3$ & $0.6 \pm 0.2$ & $0.3 \pm 0.2$ & $\cdots$ \\
\hline \multirow[t]{3}{*}{ 111210B } & LTF $(0.0-10000.0)$ & 22 & 12.4 & 35 & $-3.0 \pm 0.5$ & $0.4 \pm 0.1$ & $1.3 \pm 0.5$ & $\cdots$ \\
\hline & EXT (60.0-394.0) & 14 & 7.0 & 22 & $-2.3 \pm 0.5$ & $0.4 \pm 0.2$ & $0.09 \pm 0.06$ & $\ldots$ \\
\hline & LAT (6.5-394.0) & 18 & 10.2 & 30 & $-2.7 \pm 0.5$ & $0.6 \pm 0.2$ & $0.09 \pm 0.04$ & $\cdots$ \\
\hline \multirow[t]{3}{*}{$120107 \mathrm{~A}$} & GBM (0.1-23.1) & 8 & 7.5 & 53 & $-2.4 \pm 0.5$ & $8 \pm 3$ & $0.10 \pm 0.07$ & $\cdots$ \\
\hline & LTF $(0.0-10.0)$ & 6 & 5.7 & 47 & $-2.1 \pm 0.4$ & $13 \pm 6$ & $0.1 \pm 0.1$ & $\cdots$ \\
\hline & LAT (1.3-48.8) & 11 & 7.9 & 41 & $-1.9 \pm 0.4$ & $4 \pm 2$ & $0.2 \pm 0.2$ & $\cdots$ \\
\hline \multirow[t]{3}{*}{$120226 \mathrm{~A}$} & LTF $(0.0-500.0)$ & 84 & 20.0 & 43 & $-2.8 \pm 0.4$ & $0.7 \pm 0.2$ & $0.13 \pm 0.04$ & $\cdots$ \\
\hline & EXT (57.3-283.6) & 47 & 11.8 & 30 & $-2.9 \pm 0.6$ & $0.9 \pm 0.3$ & $0.07 \pm 0.03$ & $\cdots$ \\
\hline & LAT (29.4-283.6) & 52 & 14.0 & 37 & $-2.9 \pm 0.5$ & $1.0 \pm 0.4$ & $0.08 \pm 0.03$ & $\cdots$ \\
\hline \multirow[t]{4}{*}{$120316 \mathrm{~A}$} & GBM (1.5-28.2) & 4 & 3.0 & 25 & $-1.9 \pm 0.5$ & $2 \pm 1$ & $0.1 \pm 0.1$ & $\ldots$ \\
\hline & LTF (0.0-500.0) & 17 & 13.1 & 69 & $-2.2 \pm 0.3$ & $0.5 \pm 0.1$ & $0.2 \pm 0.1$ & $\cdots$ \\
\hline & EXT (28.2-545.0) & 15 & 11.2 & 53 & $-2.3 \pm 0.4$ & $0.4 \pm 0.1$ & $0.11 \pm 0.07$ & $\cdots$ \\
\hline & LAT (15.0-545.0) & 17 & 13.2 & 70 & $-2.2 \pm 0.3$ & $0.4 \pm 0.1$ & $0.2 \pm 0.1$ & $\cdots$ \\
\hline \multirow[t]{3}{*}{ 120420B } & LTF (0.0-4000.0) & 51 & 12.2 & 32 & $-2.2 \pm 0.3$ & $0.3 \pm 0.1$ & $1.0 \pm 0.7$ & $\cdots$ \\
\hline & $\begin{array}{r}\text { EXT } \\
(254.9-3908.0)\end{array}$ & 45 & 10.0 & 27 & $-2.1 \pm 0.4$ & $0.3 \pm 0.1$ & $1.0 \pm 0.7$ & $\cdots$ \\
\hline & $\begin{array}{r}\text { LAT } \\
(3501.7-3908.0)\end{array}$ & 28 & 9.0 & 28 & $-2.1 \pm 0.4$ & $0.5 \pm 0.2$ & $0.2 \pm 0.1$ & $\cdots$ \\
\hline \multirow[t]{4}{*}{$120526 \mathrm{~A}$} & LTF (0.0-4000.0) & 81 & 32.3 & 121 & $-1.9 \pm 0.2$ & $0.20 \pm 0.04$ & $1.2 \pm 0.7$ & $\cdots$ \\
\hline & EXT (46.7-3306.3) & 80 & 32.5 & 122 & $-1.9 \pm 0.2$ & $0.21 \pm 0.04$ & $1.1 \pm 0.6$ & $\cdots$ \\
\hline & LAT & 78 & 31.0 & 121 & $-1.8 \pm 0.2$ & $0.20 \pm 0.04$ & $0.9 \pm 0.5$ & $\cdots$ \\
\hline & $(692.2-3306.3)$ & & & & & & & \\
\hline \multirow[t]{3}{*}{ 120624B } & LTF (0.0-4000.0) & 154 & 124.3 & 750 & $-2.5 \pm 0.1$ & $2.1 \pm 0.2$ & $3.7 \pm 0.7$ & $77 \pm 8$ \\
\hline & EXT (14.3-1103.9) & 148 & 122.9 & 775 & $-2.5 \pm 0.1$ & $2.3 \pm 0.2$ & $1.1 \pm 0.2$ & $23 \pm 2$ \\
\hline & LAT (73.7-1103.9) & 148 & 122.9 & 774 & $-2.5 \pm 0.1$ & $2.3 \pm 0.2$ & $1.1 \pm 0.2$ & $22 \pm 2$ \\
\hline \multirow[t]{4}{*}{ 120709A } & GBM $(-0.1-27.2)$ & 13 & 12.8 & 126 & $-2.2 \pm 0.3$ & $8 \pm 2$ & $0.2 \pm 0.1$ & $\cdots$ \\
\hline & LTF $(0.0-10000.0)$ & 53 & 33.1 & 99 & $-2.3 \pm 0.2$ & $1.0 \pm 0.2$ & $6 \pm 2$ & $\cdots$ \\
\hline & EXT (27.2-695.9) & 36 & 18.6 & 38 & $-2.4 \pm 0.3$ & $0.6 \pm 0.2$ & $0.22 \pm 0.10$ & $\ldots$ \\
\hline & LAT (0.1-695.9) & 48 & 31.7 & 92 & $-2.3 \pm 0.2$ & $1.0 \pm 0.2$ & $0.4 \pm 0.2$ & $\cdots$ \\
\hline \multirow[t]{3}{*}{$120711 \mathrm{~A}$} & LTF $(0.0-10000.0)$ & 143 & 44.1 & 135 & $-2.1 \pm 0.2$ & $0.24 \pm 0.05$ & $1.9 \pm 0.7$ & $9 \pm 2$ \\
\hline & $\begin{array}{r}\text { EXT } \\
(106.5-5431.6)\end{array}$ & 87 & 42.5 & 161 & $-2.1 \pm 0.2$ & $0.53 \pm 0.10$ & $2.6 \pm 1.0$ & $11 \pm 2$ \\
\hline & $\begin{array}{r}\text { LAT } \\
(393.3-5431.6)\end{array}$ & 86 & 41.3 & 158 & $-2.1 \pm 0.2$ & $0.52 \pm 0.10$ & $2.5 \pm 1.0$ & $10 \pm 2$ \\
\hline \multirow[t]{2}{*}{$120729 \mathrm{~A}$} & LTF $(0.0-4000.0)$ & 16 & 6.0 & 32 & $-1.7 \pm 0.3$ & $0.4 \pm 0.2$ & $5 \pm 5$ & $3 \pm 1$ \\
\hline & EXT (24.4-432.0) & 5 & 3.1 & 31 & $-1.5 \pm 0.4$ & $3 \pm 2$ & $6 \pm 8$ & $2 \pm 1$ \\
\hline \multirow[t]{4}{*}{$120830 \mathrm{~A}$} & GBM $(0.0-0.9)$ & 3 & 3.0 & 47 & $-2.1 \pm 0.6$ & $60 \pm 40$ & $0.05 \pm 0.07$ & $\ldots$ \\
\hline & LTF (0.0-10.0) & 5 & 5.0 & 64 & $-1.8 \pm 0.4$ & $9 \pm 4$ & $0.2 \pm 0.2$ & $\cdots$ \\
\hline & EXT (0.9-10.7) & 3 & 2.9 & 29 & $-1.8 \pm 0.5$ & $5 \pm 3$ & $0.1 \pm 0.1$ & $\ldots$ \\
\hline & LAT (0.7-10.7) & 6 & 6.0 & 71 & $-1.9 \pm 0.4$ & $11 \pm 4$ & $0.1 \pm 0.1$ & $\cdots$ \\
\hline \multirow[t]{2}{*}{ 120911B } & GBM (0.0-69.0) & 50 & 49.8 & 549 & $-2.5 \pm 0.2$ & $61 \pm 9$ & $1.9 \pm 0.4$ & $\cdots$ \\
\hline & LTF $(0.0-100.0)$ & 57 & 56.7 & 608 & $-2.5 \pm 0.2$ & $47 \pm 7$ & $2.3 \pm 0.5$ & $\ldots$ \\
\hline
\end{tabular}


Table 4

(Continued)

\begin{tabular}{|c|c|c|c|c|c|c|c|c|}
\hline GRB Name & $\begin{array}{l}\text { Interval }\left(t_{0}-t_{1}\right) \\
\quad(\mathrm{s})\end{array}$ & $\begin{array}{l}\text { Trans. Ev. } \\
\text { in the ROI }\end{array}$ & $\begin{array}{l}\text { Trans. Ev. } \\
\text { Predicted }\end{array}$ & $\begin{array}{c}\text { Test } \\
\text { Statistic } \\
\text { (TS) }\end{array}$ & Spectral Index & $\begin{array}{c}\text { Flux } \\
\left(\times 10^{-5} \mathrm{~cm}^{-2} \mathrm{~s}^{-1}\right)\end{array}$ & $\begin{array}{c}\text { Fluence } \\
\left(\times 10^{-5} \mathrm{erg} \mathrm{cm}^{-2}\right)\end{array}$ & $\begin{array}{c}E_{\text {iso }}(100 \mathrm{MeV}- \\
10 \mathrm{GeV}) \\
\left(\times 10^{52} \mathrm{erg}\right)\end{array}$ \\
\hline & EXT (69.0-217.8) & 16 & 15.7 & 128 & $-2.3 \pm 0.3$ & $10 \pm 3$ & $0.8 \pm 0.4$ & $\ldots$ \\
\hline & LAT (9.2-217.8) & 65 & 64.5 & 628 & $-2.5 \pm 0.2$ & $28 \pm 4$ & $2.8 \pm 0.5$ & $\cdots$ \\
\hline \multirow[t]{2}{*}{$120915 \mathrm{~A}$} & $\operatorname{GBM}(-0.3-0.3)$ & 2 & 2.0 & 21 & $-3 \pm 1$ & $50 \pm 40$ & $0.010 \pm 0.009$ & $\cdots$ \\
\hline & LTF $(0.0-10.0)$ & 4 & 3.9 & 31 & $-2.3 \pm 0.6$ & $6 \pm 3$ & $0.04 \pm 0.04$ & $\cdots$ \\
\hline 120919B & LTF (0.0-4000.0) & 30 & 8.5 & 29 & $-2.1 \pm 0.4$ & $0.19 \pm 0.09$ & $0.7 \pm 0.6$ & $\cdots$ \\
\hline \multirow[t]{4}{*}{$121029 \mathrm{~A}$} & LTF $(0.0-4000.0)$ & 103 & 10.0 & 38 & $-1.6 \pm 0.2$ & $0.08 \pm 0.03$ & $1.2 \pm 0.9$ & $\ldots$ \\
\hline & EXT (14.9-1926.5) & 88 & 9.4 & 38 & $-1.6 \pm 0.3$ & $0.08 \pm 0.04$ & $0.6 \pm 0.5$ & $\ldots$ \\
\hline & LAT & 82 & 6.4 & 23 & $-1.6 \pm 0.3$ & $0.06 \pm 0.03$ & $0.4 \pm 0.4$ & $\cdots$ \\
\hline & $(119.0-1926.5)$ & & & & & & & \\
\hline \multirow[t]{3}{*}{ 121123B } & LTF $(0.0-4000.0)$ & 32 & 10.6 & 27 & $-2.3 \pm 0.4$ & $0.19 \pm 0.08$ & $0.5 \pm 0.3$ & $\cdots$ \\
\hline & EXT (44.8-1651.3) & 26 & 9.9 & 25 & $-2.3 \pm 0.4$ & $0.23 \pm 0.10$ & $0.2 \pm 0.1$ & $\ldots$ \\
\hline & $\begin{array}{r}\text { LAT } \\
(268.0-1651.3)\end{array}$ & 24 & 10.0 & 26 & $-2.4 \pm 0.4$ & $0.2 \pm 0.1$ & $0.2 \pm 0.1$ & $\cdots$ \\
\hline \multirow[t]{3}{*}{$130310 \mathrm{~A}$} & LTF $(0.0-4000.0)$ & 38 & 15.3 & 53 & $-1.9 \pm 0.2$ & $0.5 \pm 0.1$ & $3 \pm 2$ & $\cdots$ \\
\hline & EXT (20.1-560.1) & 26 & 15.3 & 54 & $-2.1 \pm 0.3$ & $0.7 \pm 0.2$ & $0.4 \pm 0.2$ & $\ldots$ \\
\hline & LAT (67.2-560.1) & 25 & 14.1 & 48 & $-2.1 \pm 0.3$ & $0.7 \pm 0.2$ & $0.3 \pm 0.2$ & $\cdots$ \\
\hline \multirow[t]{4}{*}{$130325 \mathrm{~A}$} & LTF $(0.0-10000.0)$ & 277 & 27.2 & 64 & $-2.1 \pm 0.2$ & $0.08 \pm 0.02$ & $0.8 \pm 0.4$ & $\cdots$ \\
\hline & EXT (7.7-1029.7) & 60 & 8.5 & 36 & $-1.7 \pm 0.3$ & $0.13 \pm 0.06$ & $0.4 \pm 0.3$ & $\ldots$ \\
\hline & LAT & 43 & 5.9 & 33 & $-1.5 \pm 0.3$ & $0.12 \pm 0.06$ & $0.4 \pm 0.4$ & $\ldots$ \\
\hline & $(324.1-1029.7)$ & & & & & & & \\
\hline \multirow[t]{4}{*}{ 130327B } & GBM (2.0-33.3) & 12 & 10.1 & 68 & $-1.8 \pm 0.3$ & $7 \pm 2$ & $0.3 \pm 0.3$ & $\cdots$ \\
\hline & LTF $(0.0-100.0)$ & 44 & 39.5 & 328 & $-1.7 \pm 0.1$ & $8 \pm 1$ & $1.8 \pm 0.8$ & $\cdots$ \\
\hline & EXT (33.3-523.2) & 111 & 45.2 & 205 & $-1.8 \pm 0.1$ & $1.4 \pm 0.2$ & $1.5 \pm 0.7$ & $\ldots$ \\
\hline & LAT (8.0-523.2) & 123 & 55.0 & 251 & $-1.8 \pm 0.1$ & $1.6 \pm 0.2$ & $1.7 \pm 0.7$ & $\cdots$ \\
\hline \multirow[t]{5}{*}{$130427 \mathrm{~A}$} & GBM (4.1-142.3) & 283 & 270.8 & 2765 & $-1.90 \pm 0.05$ & $52 \pm 3$ & $10 \pm 1$ & $1.7 \pm 0.1$ \\
\hline & LTF $(0.0-500.0)$ & 558 & 518.4 & 4514 & $-1.97 \pm 0.04$ & $22 \pm 1$ & $13 \pm 2$ & $2.5 \pm 0.2$ \\
\hline & EXT & 589 & 368.2 & 1397 & $-2.12 \pm 0.06$ & $0.68 \pm 0.04$ & $19 \pm 2$ & $4.5 \pm 0.3$ \\
\hline & (142.3-34366.2) & & & & & & & \\
\hline & LAT $(0.1-34366.2)$ & 877 & 635.9 & 2795 & $-1.99 \pm 0.04$ & $1.13 \pm 0.05$ & $45 \pm 4$ & $8.6 \pm 0.4$ \\
\hline \multirow[t]{4}{*}{ 130502B } & GBM (7.2-31.5) & 21 & 20.9 & 219 & $-2.2 \pm 0.2$ & $26 \pm 6$ & $0.5 \pm 0.2$ & $\cdots$ \\
\hline & LTF $(0.0-500.0)$ & 84 & 72.0 & 407 & $-2.0 \pm 0.1$ & $4.3 \pm 0.6$ & $2.2 \pm 0.8$ & $\ldots$ \\
\hline & EXT (31.5-1316.6) & 80 & 55.3 & 238 & $-2.0 \pm 0.1$ & $1.1 \pm 0.2$ & $1.6 \pm 0.7$ & $\ldots$ \\
\hline & LAT (12.4-1316.6) & 101 & 74.9 & 345 & $-2.0 \pm 0.1$ & $1.5 \pm 0.2$ & $2.1 \pm 0.8$ & $\cdots$ \\
\hline \multirow[t]{4}{*}{$130504 \mathrm{C}$} & GBM (8.7-81.9) & 8 & 7.4 & 29 & $-2.5 \pm 0.5$ & $3 \pm 1$ & $0.10 \pm 0.05$ & $\cdots$ \\
\hline & LTF $(0.0-500.0)$ & 67 & 23.3 & 104 & $-1.9 \pm 0.2$ & $0.8 \pm 0.2$ & $0.6 \pm 0.3$ & $\cdots$ \\
\hline & EXT (81.9-590.3) & 69 & 18.2 & 84 & $-1.9 \pm 0.2$ & $0.6 \pm 0.2$ & $0.5 \pm 0.3$ & $\ldots$ \\
\hline & LAT (42.6-590.3) & 75 & 23.0 & 103 & $-1.9 \pm 0.2$ & $0.7 \pm 0.2$ & $0.5 \pm 0.3$ & $\cdots$ \\
\hline \multirow[t]{4}{*}{$130518 \mathrm{~A}$} & GBM (9.9-58.5) & 12 & 10.8 & 54 & $-3.5 \pm 0.7$ & $6 \pm 2$ & $0.07 \pm 0.03$ & $7 \pm 5$ \\
\hline & LTF $(0.0-500.0)$ & 62 & 38.8 & 165 & $-2.7 \pm 0.3$ & $2.8 \pm 0.5$ & $0.5 \pm 0.1$ & $18 \pm 5$ \\
\hline & EXT (58.5-343.6) & 45 & 28.9 & 136 & $-2.7 \pm 0.3$ & $2.7 \pm 0.6$ & $0.29 \pm 0.08$ & $10 \pm 3$ \\
\hline & LAT (26.8-343.6) & 56 & 38.4 & 174 & $-2.9 \pm 0.3$ & $3.2 \pm 0.6$ & $0.35 \pm 0.08$ & $15 \pm 5$ \\
\hline \multirow[t]{3}{*}{ 130606B } & LTF $(0.0-4000.0)$ & 36 & 18.1 & 96 & $-1.8 \pm 0.2$ & $0.6 \pm 0.2$ & $5 \pm 4$ & $\cdots$ \\
\hline & EXT (57.6-527.3) & 27 & 17.0 & 96 & $-1.7 \pm 0.2$ & $0.9 \pm 0.3$ & $1.0 \pm 0.8$ & $\cdots$ \\
\hline & LAT (130.5-527.3) & 27 & 16.9 & 96 & $-1.7 \pm 0.2$ & $0.9 \pm 0.3$ & $0.8 \pm 0.6$ & $\cdots$ \\
\hline $130702 \mathrm{~A}$ & LTF $(0.0-4000.0)$ & 12 & 7.9 & 26 & $-2.0 \pm 0.3$ & $0.13 \pm 0.05$ & $0.6 \pm 0.5$ & $0.019 \pm 0.009$ \\
\hline \multirow[t]{4}{*}{$130821 \mathrm{~A}$} & GBM (3.6-90.6) & 12 & 8.5 & 52 & $-2.6 \pm 0.5$ & $2.0 \pm 0.7$ & $0.08 \pm 0.04$ & $\cdots$ \\
\hline & LTF $(0.0-10000.0)$ & 252 & 81.9 & 202 & $-2.4 \pm 0.2$ & $0.27 \pm 0.04$ & $1.4 \pm 0.3$ & $\ldots$ \\
\hline & EXT (90.6-6103.7) & 173 & 64.7 & 173 & $-2.3 \pm 0.2$ & $0.34 \pm 0.08$ & $1.2 \pm 0.3$ & $\cdots$ \\
\hline & LAT (33.9-6103.7) & 183 & 71.9 & 198 & $-2.4 \pm 0.2$ & $0.37 \pm 0.05$ & $1.2 \pm 0.3$ & $\cdots$ \\
\hline \multirow[t]{4}{*}{$130828 \mathrm{~A}$} & GBM (13.3-150.3) & 34 & 32.7 & 290 & $-2.4 \pm 0.2$ & $6 \pm 1$ & $0.5 \pm 0.2$ & $\cdots$ \\
\hline & LTF $(0.0-100.0)$ & 33 & 31.7 & 287 & $-2.3 \pm 0.2$ & $7 \pm 1$ & $0.4 \pm 0.1$ & $\cdots$ \\
\hline & EXT (150.3-616.8) & 23 & 6.9 & 23 & $-2.0 \pm 0.4$ & $0.2 \pm 0.1$ & $0.1 \pm 0.1$ & $\cdots$ \\
\hline & LAT (17.1-616.8) & 57 & 40.3 & 145 & $-2.2 \pm 0.2$ & $1.2 \pm 0.2$ & $0.5 \pm 0.2$ & $\cdots$ \\
\hline
\end{tabular}


Table 4

(Continued)

\begin{tabular}{|c|c|c|c|c|c|c|c|c|}
\hline GRB Name & $\begin{array}{l}\text { Interval }\left(t_{0}-t_{1}\right) \\
\quad(\mathrm{s})\end{array}$ & $\begin{array}{l}\text { Trans. Ev. } \\
\text { in the ROI }\end{array}$ & $\begin{array}{l}\text { Trans. Ev. } \\
\text { Predicted }\end{array}$ & $\begin{array}{c}\text { Test } \\
\text { Statistic } \\
\text { (TS) }\end{array}$ & Spectral Index & $\begin{array}{c}\text { Flux } \\
\left(\times 10^{-5} \mathrm{~cm}^{-2} \mathrm{~s}^{-1}\right)\end{array}$ & $\begin{array}{c}\text { Fluence } \\
\left(\times 10^{-5} \mathrm{erg} \mathrm{cm}^{-2}\right)\end{array}$ & $\begin{array}{c}E_{\text {iso }}(100 \mathrm{MeV}- \\
10 \mathrm{GeV}) \\
\left(\times 10^{52} \mathrm{erg}\right)\end{array}$ \\
\hline \multirow[t]{2}{*}{$130907 \mathrm{~A}$} & LTF $(0.0-10000.0)$ & 48 & 10.6 & 34 & $-2.1 \pm 0.4$ & $0.08 \pm 0.04$ & $0.7 \pm 0.5$ & $2.4 \pm 0.9$ \\
\hline & $\begin{array}{r}\text { EXT } \\
(210.0-4010.9)\end{array}$ & 12 & 3.6 & 20 & $-1.8 \pm 0.5$ & $0.14 \pm 0.10$ & $1 \pm 1$ & $2 \pm 1$ \\
\hline \multirow[t]{4}{*}{$131014 \mathrm{~A}$} & GBM (1.0-4.2) & 10 & 10.0 & 132 & $-2.0 \pm 0.3$ & $400 \pm 100$ & $1 \pm 1$ & $\cdots$ \\
\hline & LTF $(0.0-10.0)$ & 12 & 11.9 & 132 & $-1.9 \pm 0.3$ & $140 \pm 40$ & $2 \pm 1$ & $\ldots$ \\
\hline & EXT (4.2-200.4) & 19 & 15.5 & 82 & $-2.0 \pm 0.2$ & $4 \pm 1$ & $0.9 \pm 0.6$ & $\ldots$ \\
\hline & LAT (1.9-200.4) & 29 & 25.1 & 155 & $-1.9 \pm 0.2$ & $6 \pm 1$ & $1.7 \pm 1.0$ & $\ldots$ \\
\hline \multirow[t]{4}{*}{$131029 \mathrm{~A}$} & GBM (1.0-105.5) & 23 & 22.9 & 223 & $-2.6 \pm 0.3$ & $13 \pm 3$ & $0.6 \pm 0.2$ & $\ldots$ \\
\hline & LTF $(0.0-500.0)$ & 46 & 36.2 & 177 & $-2.4 \pm 0.2$ & $2.4 \pm 0.5$ & $0.6 \pm 0.2$ & $\cdots$ \\
\hline & EXT (105.5-557.2) & 24 & 13.2 & 35 & $-2.3 \pm 0.3$ & $0.8 \pm 0.3$ & $0.2 \pm 0.1$ & $\cdots$ \\
\hline & LAT (38.7-557.2) & 47 & 37.2 & 185 & $-2.4 \pm 0.2$ & $2.1 \pm 0.4$ & $0.6 \pm 0.2$ & $\cdots$ \\
\hline \multirow[t]{4}{*}{$131108 \mathrm{~A}$} & GBM $(0.3-18.5)$ & 136 & 135.4 & 1695 & $-2.6 \pm 0.1$ & $120 \pm 10$ & $0.9 \pm 0.1$ & $24 \pm 3$ \\
\hline & LTF $(0.0-10.0)$ & 136 & 135.8 & 1857 & $-2.6 \pm 0.1$ & $210 \pm 20$ & $0.9 \pm 0.1$ & $24 \pm 3$ \\
\hline & EXT (18.5-678.1) & 60 & 43.0 & 112 & $-2.8 \pm 0.3$ & $1.0 \pm 0.2$ & $0.24 \pm 0.05$ & $9 \pm 2$ \\
\hline & LAT (0.0-678.1) & 223 & 202.0 & 1321 & $-2.7 \pm 0.1$ & $4.6 \pm 0.3$ & $1.2 \pm 0.1$ & $37 \pm 4$ \\
\hline \multirow[t]{3}{*}{$131209 \mathrm{~A}$} & LTF (0.0-500.0) & 38 & 12.8 & 28 & $-3.5 \pm 0.7$ & $0.6 \pm 0.2$ & $0.08 \pm 0.03$ & $\cdots$ \\
\hline & EXT (16.4-374.8) & 30 & 9.3 & 22 & $-3.3 \pm 0.8$ & $0.6 \pm 0.2$ & $0.06 \pm 0.03$ & $\ldots$ \\
\hline & LAT (14.3-374.8) & 32 & 11.4 & 27 & $-3.3 \pm 0.7$ & $0.7 \pm 0.2$ & $0.07 \pm 0.03$ & $\cdots$ \\
\hline \multirow[t]{4}{*}{$131231 \mathrm{~A}$} & GBM (13.3-44.5) & 5 & 4.8 & 31 & $-2.7 \pm 0.7$ & $4 \pm 2$ & $0.05 \pm 0.03$ & $0.07 \pm 0.03$ \\
\hline & LTF $(0.0-4000.0)$ & 66 & 40.9 & 261 & $-1.7 \pm 0.1$ & $0.6 \pm 0.1$ & $6 \pm 3$ & $2.4 \pm 0.6$ \\
\hline & EXT (44.5-4824.2) & 80 & 36.5 & 244 & $-1.6 \pm 0.1$ & $0.28 \pm 0.05$ & $4 \pm 2$ & $1.5 \pm 0.4$ \\
\hline & LAT (23.1-4824.2) & 84 & 40.0 & 256 & $-1.7 \pm 0.1$ & $0.31 \pm 0.06$ & $4 \pm 2$ & $1.6 \pm 0.3$ \\
\hline \multirow[t]{3}{*}{$140102 \mathrm{~A}$} & LTF $(0.0-100.0)$ & 18 & 13.8 & 77 & $-2.1 \pm 0.3$ & $2.9 \pm 0.9$ & $0.2 \pm 0.1$ & $\cdots$ \\
\hline & EXT (4.1-60.2) & 12 & 11.6 & 104 & $-2.1 \pm 0.3$ & $4 \pm 1$ & $0.2 \pm 0.1$ & $\ldots$ \\
\hline & LAT (3.1-60.2) & 14 & 13.6 & 119 & $-2.1 \pm 0.3$ & $5 \pm 1$ & $0.2 \pm 0.1$ & $\cdots$ \\
\hline \multirow[t]{4}{*}{ 140104B } & LTF (0.0-4000.0) & 86 & 23.0 & 72 & $-2.0 \pm 0.2$ & $0.21 \pm 0.06$ & $1.0 \pm 0.5$ & $\cdots$ \\
\hline & EXT & 41 & 18.8 & 73 & $-2.0 \pm 0.2$ & $0.4 \pm 0.1$ & $0.4 \pm 0.3$ & $\ldots$ \\
\hline & $(198.1-1174.0)$ & & & & & & & \\
\hline & $\begin{array}{r}\text { LAT } \\
(227.3-1174.0)\end{array}$ & 36 & 17.7 & 72 & $-2.0 \pm 0.2$ & $0.4 \pm 0.1$ & $0.4 \pm 0.2$ & $\cdots$ \\
\hline \multirow[t]{4}{*}{ 140110A } & GBM $(-0.3-9.2)$ & 29 & 28.8 & 330 & $-2.6 \pm 0.3$ & $60 \pm 10$ & $0.22 \pm 0.07$ & $\ldots$ \\
\hline & LTF $(0.0-10.0)$ & 30 & 29.8 & 339 & $-2.6 \pm 0.3$ & $50 \pm 10$ & $0.22 \pm 0.06$ & $\ldots$ \\
\hline & EXT (9.2-159.4) & 14 & 9.5 & 31 & $-2.3 \pm 0.4$ & $1.0 \pm 0.4$ & $0.09 \pm 0.06$ & $\ldots$ \\
\hline & LAT (0.6-159.4) & 42 & 37.9 & 231 & $-2.6 \pm 0.3$ & $3.9 \pm 0.7$ & $0.25 \pm 0.07$ & $\cdots$ \\
\hline $140124 \mathrm{~A}$ & LTF $(0.0-500.0)$ & 33 & 5.4 & 26 & $-1.6 \pm 0.3$ & $0.18 \pm 0.10$ & $0.4 \pm 0.4$ & $\cdots$ \\
\hline \multirow{5}{*}{ 140206B } & GBM (7.5-154.2) & 25 & 24.0 & 183 & $-2.8 \pm 0.3$ & $4.2 \pm 0.9$ & $0.22 \pm 0.07$ & $\ldots$ \\
\hline & LTF $(0.0-10000.0)$ & 245 & 87.2 & 302 & $-2.1 \pm 0.1$ & $0.30 \pm 0.04$ & $2.6 \pm 0.7$ & $\ldots$ \\
\hline & EXT & 218 & 62.9 & 239 & $-1.9 \pm 0.1$ & $0.22 \pm 0.03$ & $2.3 \pm 0.7$ & $\cdots$ \\
\hline & (154.2-8585.0) & & & & & & & \\
\hline & LAT (0.6-8585.0) & 244 & 85.8 & 298 & $-2.1 \pm 0.1$ & $0.30 \pm 0.04$ & $2.3 \pm 0.6$ & $\cdots$ \\
\hline 140219A & LTF $(0.0-4000.0)$ & 43 & 12.2 & 31 & $-2.2 \pm 0.3$ & $0.19 \pm 0.07$ & $0.6 \pm 0.4$ & $\cdots$ \\
\hline \multirow{2}{*}{$140323 \mathrm{~A}$} & LTF (0.0-4000.0) & 15 & 6.8 & 40 & $-1.5 \pm 0.3$ & $0.17 \pm 0.09$ & $3 \pm 3$ & $\cdots$ \\
\hline & EXT (116.5-715.6) & 12 & 4.1 & 21 & $-1.6 \pm 0.4$ & $0.14 \pm 0.09$ & $0.4 \pm 0.4$ & $\cdots$ \\
\hline \multirow[t]{4}{*}{$140402 \mathrm{~A}$} & GBM $(-0.1-0.2)$ & 4 & 4.0 & 61 & $-2.4 \pm 0.6$ & $180 \pm 90$ & $0.03 \pm 0.03$ & $\ldots$ \\
\hline & LTF $(0.0-100.0)$ & 21 & 14.3 & 92 & $-1.8 \pm 0.2$ & $2.0 \pm 0.6$ & $0.4 \pm 0.3$ & $\ldots$ \\
\hline & EXT $(0.2-71.7)$ & 16 & 9.4 & 62 & $-1.7 \pm 0.3$ & $1.8 \pm 0.7$ & $0.3 \pm 0.3$ & $\ldots$ \\
\hline & LAT (0.1-71.7) & 18 & 10.4 & 76 & $-1.8 \pm 0.3$ & $2.0 \pm 0.8$ & $0.3 \pm 0.3$ & $\cdots$ \\
\hline \multirow[t]{2}{*}{$140416 \mathrm{~A}$} & LTF $(0.0-4000.0)$ & 64 & 13.2 & 35 & $-2.0 \pm 0.3$ & $0.17 \pm 0.07$ & $0.7 \pm 0.5$ & $\cdots$ \\
\hline & EXT (29.0-2207.4) & 46 & 14.1 & 22 & $-2.4 \pm 0.4$ & $0.24 \pm 0.08$ & $0.3 \pm 0.1$ & $\ldots$ \\
\hline \multirow[t]{4}{*}{$140523 \mathrm{~A}$} & GBM (0.6-19.8) & 15 & 14.8 & 160 & $-1.9 \pm 0.2$ & $28 \pm 7$ & $0.8 \pm 0.5$ & $\cdots$ \\
\hline & LTF (0.0-500.0) & 44 & 42.6 & 368 & $-2.0 \pm 0.1$ & $4.7 \pm 0.8$ & $2 \pm 1$ & $\cdots$ \\
\hline & EXT (19.8-470.2) & 26 & 24.9 & 197 & $-2.0 \pm 0.2$ & $3.0 \pm 0.6$ & $1.4 \pm 0.6$ & $\cdots$ \\
\hline & LAT (1.6-470.2) & 41 & 39.6 & 343 & $-2.0 \pm 0.1$ & $4.5 \pm 0.8$ & $2 \pm 1$ & $\cdots$ \\
\hline
\end{tabular}


Table 4

(Continued)

\begin{tabular}{|c|c|c|c|c|c|c|c|c|}
\hline GRB Name & $\begin{array}{l}\text { Interval }\left(t_{0}-t_{1}\right) \\
\quad(\mathrm{s})\end{array}$ & $\begin{array}{l}\text { Trans. Ev. } \\
\text { in the ROI }\end{array}$ & $\begin{array}{l}\text { Trans. Ev. } \\
\text { Predicted }\end{array}$ & $\begin{array}{c}\text { Test } \\
\text { Statistic } \\
\text { (TS) }\end{array}$ & Spectral Index & $\begin{array}{c}\text { Flux } \\
\left(\times 10^{-5} \mathrm{~cm}^{-2} \mathrm{~s}^{-1}\right)\end{array}$ & $\begin{array}{c}\text { Fluence } \\
\left(\times 10^{-5} \mathrm{erg} \mathrm{cm}^{-2}\right)\end{array}$ & $\begin{array}{c}E_{\text {iso }}(100 \mathrm{MeV}- \\
10 \mathrm{GeV}) \\
\left(\times 10^{52} \mathrm{erg}\right)\end{array}$ \\
\hline \multirow[t]{3}{*}{$140528 \mathrm{~A}$} & LTF $(0.0-4000.0)$ & 119 & 19.2 & 36 & $-2.1 \pm 0.3$ & $0.14 \pm 0.05$ & $0.5 \pm 0.3$ & $\cdots$ \\
\hline & EXT (14.6-1377.9) & 67 & 14.0 & 34 & $-2.0 \pm 0.3$ & $0.18 \pm 0.07$ & $0.3 \pm 0.2$ & $\ldots$ \\
\hline & LAT (81.1-1377.9) & 66 & 14.1 & 34 & $-2.0 \pm 0.3$ & $0.19 \pm 0.07$ & $0.3 \pm 0.2$ & $\cdots$ \\
\hline \multirow[t]{4}{*}{ 140619B } & GBM $(-0.3-2.6)$ & 21 & 21.0 & 314 & $-2.0 \pm 0.2$ & $140 \pm 30$ & $0.5 \pm 0.3$ & $\cdots$ \\
\hline & LTF $(0.0-10.0)$ & 25 & 24.9 & 314 & $-1.9 \pm 0.2$ & $47 \pm 9$ & $0.6 \pm 0.3$ & $\ldots$ \\
\hline & EXT (2.6-5.3) & 3 & 2.9 & 28 & $-1.7 \pm 0.4$ & $20 \pm 10$ & $0.1 \pm 0.2$ & $\cdots$ \\
\hline & LAT (0.2-5.3) & 23 & 22.9 & 319 & $-1.9 \pm 0.2$ & $80 \pm 20$ & $0.6 \pm 0.3$ & $\cdots$ \\
\hline \multirow[t]{4}{*}{$140723 \mathrm{~A}$} & GBM (0.0-56.3) & 31 & 26.1 & 113 & $-2.2 \pm 0.2$ & $13 \pm 3$ & $0.5 \pm 0.2$ & $\cdots$ \\
\hline & LTF $(0.0-100.0)$ & 33 & 32.4 & 270 & $-2.2 \pm 0.2$ & $9 \pm 2$ & $0.6 \pm 0.2$ & $\ldots$ \\
\hline & EXT (56.3-103.8) & 3 & 3.0 & 25 & $-1.8 \pm 0.5$ & $1.6 \pm 0.9$ & $0.1 \pm 0.2$ & $\cdots$ \\
\hline & LAT (0.6-103.8) & 34 & 33.4 & 282 & $-2.2 \pm 0.2$ & $9 \pm 2$ & $0.7 \pm 0.2$ & $\cdots$ \\
\hline \multirow[t]{3}{*}{ 140729A } & GBM (0.5-56.1) & 11 & 9.5 & 64 & $-1.9 \pm 0.3$ & $2.5 \pm 0.9$ & $0.2 \pm 0.2$ & $\ldots$ \\
\hline & LTF $(0.0-100.0)$ & 15 & 11.9 & 68 & $-1.8 \pm 0.2$ & $1.7 \pm 0.5$ & $0.3 \pm 0.3$ & $\ldots$ \\
\hline & LAT (4.8-58.9) & 10 & 8.5 & 57 & $-1.8 \pm 0.3$ & $2.3 \pm 0.8$ & $0.2 \pm 0.2$ & $\cdots$ \\
\hline \multirow[t]{5}{*}{ 140810A } & LTF (0.0-4000.0) & 49 & 13.6 & 82 & $-1.7 \pm 0.3$ & $0.11 \pm 0.04$ & $1 \pm 1$ & $\cdots$ \\
\hline & EXT & 152 & 13.5 & 73 & $-1.5 \pm 0.2$ & $0.03 \pm 0.01$ & $3 \pm 2$ & $\cdots$ \\
\hline & $(88.3-18827.5)$ & & & & & & & \\
\hline & LAT & 150 & 13.0 & 72 & $-1.5 \pm 0.2$ & $0.03 \pm 0.01$ & $3 \pm 2$ & $\cdots$ \\
\hline & $(460.8-18827.5)$ & & & & & & & \\
\hline \multirow[t]{4}{*}{$140928 \mathrm{~A}$} & LTF $(0.0-4000.0)$ & 35 & 6.6 & 61 & $-1.2 \pm 0.3$ & $0.06 \pm 0.03$ & $3 \pm 3$ & $\cdots$ \\
\hline & EXT (7.2-2554.7) & 19 & 7.0 & 22 & $-2.1 \pm 0.4$ & $0.14 \pm 0.07$ & $0.3 \pm 0.3$ & $\ldots$ \\
\hline & LAT & 16 & 5.2 & 21 & $-1.9 \pm 0.5$ & $0.11 \pm 0.07$ & $0.1 \pm 0.1$ & $\ldots$ \\
\hline & $(1676.6-2554.7)$ & & & & & & & \\
\hline \multirow[t]{4}{*}{$141012 \mathrm{~A}$} & GBM $(-25.9-11.8)$ & 8 & 7.7 & 61 & $-2.4 \pm 0.4$ & $7 \pm 2$ & $0.13 \pm 0.08$ & $\cdots$ \\
\hline & LTF $(0.0-10000.0)$ & 21 & 17.6 & 120 & $-2.1 \pm 0.3$ & $4 \pm 1$ & $30 \pm 20$ & $\ldots$ \\
\hline & EXT (11.8-107.0) & 12 & 10.5 & 79 & $-1.9 \pm 0.3$ & $3 \pm 1$ & $0.4 \pm 0.3$ & $\cdots$ \\
\hline & LAT(3.1-107.0) & 16 & 14.5 & 118 & $-2.0 \pm 0.2$ & $4 \pm 1$ & $0.5 \pm 0.3$ & $\cdots$ \\
\hline \multirow[t]{4}{*}{$141028 \mathrm{~A}$} & GBM (6.7-38.1) & 20 & 18.9 & 169 & $-3.2 \pm 0.5$ & $13 \pm 3$ & $0.12 \pm 0.03$ & $7 \pm 3$ \\
\hline & LTF $(0.0-500.0)$ & 40 & 34.8 & 156 & $-2.5 \pm 0.2$ & $1.9 \pm 0.3$ & $0.4 \pm 0.1$ & $10 \pm 2$ \\
\hline & EXT (38.1-500.5) & 20 & 15.7 & 90 & $-2.0 \pm 0.2$ & $0.9 \pm 0.2$ & $0.4 \pm 0.3$ & $4 \pm 1$ \\
\hline & LAT (10.9-500.5) & 39 & 33.8 & 152 & $-2.4 \pm 0.2$ & $1.8 \pm 0.3$ & $0.5 \pm 0.1$ & $9 \pm 2$ \\
\hline \multirow[t]{3}{*}{$141102 \mathrm{~A}$} & $\operatorname{LTF}(0.0-100.0)$ & 15 & 9.9 & 33 & $-2.4 \pm 0.4$ & $3 \pm 1$ & $0.15 \pm 0.09$ & $\cdots$ \\
\hline & EXT (2.6-34.5) & 8 & 6.4 & 49 & $-2.0 \pm 0.4$ & $6 \pm 2$ & $0.2 \pm 0.2$ & $\cdots$ \\
\hline & LAT $(3.5-34.5)$ & 7 & 5.3 & 40 & $-1.9 \pm 0.4$ & $5 \pm 2$ & $0.2 \pm 0.2$ & $\cdots$ \\
\hline \multirow[t]{3}{*}{$141113 \mathrm{~A}$} & GBM $(-0.1-0.4)$ & 4 & 4.0 & 60 & $-2.1 \pm 0.5$ & $200 \pm 100$ & $0.1 \pm 0.1$ & $\cdots$ \\
\hline & $\operatorname{LTF}(0.0-100.0)$ & 8 & 7.6 & 37 & $-2.1 \pm 0.4$ & $1.6 \pm 0.6$ & $0.1 \pm 0.1$ & $\cdots$ \\
\hline & LAT (0.1-3.5) & 4 & 4.0 & 40 & $-2.6 \pm 0.7$ & $30 \pm 20$ & $0.05 \pm 0.04$ & $\cdots$ \\
\hline \multirow[t]{4}{*}{$141207 \mathrm{~A}$} & GBM (1.3-22.3) & 39 & 38.8 & 495 & $-1.9 \pm 0.1$ & $70 \pm 10$ & $2.0 \pm 0.8$ & $\cdots$ \\
\hline & LTF $(0.0-100.0)$ & 47 & 44.9 & 414 & $-2.0 \pm 0.1$ & $16 \pm 2$ & $1.8 \pm 0.7$ & $\ldots$ \\
\hline & EXT (22.3-734.3) & 29 & 8.7 & 32 & $-1.8 \pm 0.3$ & $0.2 \pm 0.1$ & $0.3 \pm 0.3$ & $\cdots$ \\
\hline & LAT (1.8-734.3) & 67 & 46.9 & 197 & $-1.8 \pm 0.1$ & $1.2 \pm 0.2$ & $1.7 \pm 0.6$ & $\cdots$ \\
\hline \multirow[t]{3}{*}{ 141221B } & LTF $(0.0-100.0)$ & 12 & 7.0 & 41 & $-1.9 \pm 0.4$ & $1.2 \pm 0.5$ & $0.2 \pm 0.2$ & $\cdots$ \\
\hline & EXT (31.2-58.5) & 5 & 3.6 & 40 & $-1.5 \pm 0.4$ & $2 \pm 1$ & $0.3 \pm 0.4$ & $\ldots$ \\
\hline & LAT (22.2-58.5) & 8 & 4.5 & 39 & $-1.6 \pm 0.4$ & $2 \pm 1$ & $0.3 \pm 0.3$ & $\ldots$ \\
\hline \multirow[t]{2}{*}{$141222 \mathrm{~A}$} & EXT (2.8-440.6) & 67 & 15.0 & 39 & $-2.1 \pm 0.3$ & $0.8 \pm 0.3$ & $0.3 \pm 0.2$ & $\ldots$ \\
\hline & LAT (34.2-440.6) & 67 & 15.0 & 39 & $-2.1 \pm 0.3$ & $0.8 \pm 0.3$ & $0.3 \pm 0.2$ & $\cdots$ \\
\hline 150118B & LTF $(0.0-500.0)$ & 10 & 7.6 & 30 & $-2.5 \pm 0.5$ & $9 \pm 4$ & $2 \pm 1$ & $\cdots$ \\
\hline 150202B & LTF $(0.0-4000.0)$ & 57 & 11.8 & 29 & $-2.2 \pm 0.4$ & $0.16 \pm 0.07$ & $0.5 \pm 0.3$ & $\ldots$ \\
\hline \multirow[t]{3}{*}{$150210 \mathrm{~A}$} & GBM $(0.0-31.3)$ & 16 & 15.2 & 157 & $-2.2 \pm 0.3$ & $13 \pm 3$ & $0.3 \pm 0.2$ & $\ldots$ \\
\hline & LTF $(0.0-10.0)$ & 14 & 13.1 & 112 & $-2.3 \pm 0.3$ & $40 \pm 10$ & $0.2 \pm 0.1$ & $\ldots$ \\
\hline & LAT (0.8-169.4) & 23 & 20.5 & 77 & $-2.2 \pm 0.3$ & $3.3 \pm 0.8$ & $0.4 \pm 0.2$ & $\cdots$ \\
\hline $150314 \mathrm{~A}$ & LTF $(0.0-500.0)$ & 34 & 17.6 & 38 & $-2.7 \pm 0.4$ & $0.7 \pm 0.2$ & $0.15 \pm 0.06$ & $2.2 \pm 0.7$ \\
\hline
\end{tabular}


Table 4

(Continued)

\begin{tabular}{|c|c|c|c|c|c|c|c|c|}
\hline GRB Name & $\begin{array}{l}\text { Interval }\left(t_{0}-t_{1}\right) \\
\quad(\mathrm{s})\end{array}$ & $\begin{array}{l}\text { Trans. Ev. } \\
\text { in the ROI }\end{array}$ & $\begin{array}{l}\text { Trans. Ev. } \\
\text { Predicted }\end{array}$ & $\begin{array}{c}\text { Test } \\
\text { Statistic } \\
\text { (TS) }\end{array}$ & Spectral Index & $\begin{array}{c}\text { Flux } \\
\left(\times 10^{-5} \mathrm{~cm}^{-2} \mathrm{~s}^{-1}\right)\end{array}$ & $\begin{array}{c}\text { Fluence } \\
\left(\times 10^{-5} \mathrm{erg} \mathrm{cm}^{-2}\right)\end{array}$ & $\begin{array}{c}E_{\text {iso }}(100 \mathrm{MeV}- \\
10 \mathrm{GeV}) \\
\left(\times 10^{52} \mathrm{erg}\right)\end{array}$ \\
\hline & LAT $(0.1-3064.3)$ & 137 & 18.3 & 23 & $-2.5 \pm 0.4$ & $0.10 \pm 0.04$ & $0.15 \pm 0.06$ & $1.8 \pm 0.7$ \\
\hline \multirow{3}{*}{$150403 \mathrm{~A}$} & LTF $(0.0-4000.0)$ & 101 & 19.0 & 49 & $-1.9 \pm 0.2$ & $0.10 \pm 0.03$ & $0.5 \pm 0.3$ & $3.2 \pm 1.0$ \\
\hline & EXT (25.6-970.5) & 32 & 8.6 & 36 & $-1.7 \pm 0.3$ & $0.18 \pm 0.08$ & $0.4 \pm 0.4$ & $1.4 \pm 0.6$ \\
\hline & LAT (399.5-970.5) & 27 & 8.5 & 38 & $-1.7 \pm 0.3$ & $0.22 \pm 0.10$ & $0.3 \pm 0.3$ & $1.1 \pm 0.4$ \\
\hline \multirow[t]{4}{*}{$150510 \mathrm{~A}$} & GBM $(0.4-52.3)$ & 8 & 6.9 & 46 & $-2.4 \pm 0.5$ & $5 \pm 2$ & $0.12 \pm 0.08$ & $\cdots$ \\
\hline & LTF $(0.0-500.0)$ & 22 & 11.4 & 56 & $-1.9 \pm 0.3$ & $0.7 \pm 0.2$ & $0.6 \pm 0.4$ & $\cdots$ \\
\hline & EXT (52.3-147.6) & 7 & 4.6 & 36 & $-1.7 \pm 0.4$ & $1.5 \pm 0.8$ & $0.3 \pm 0.4$ & $\cdots$ \\
\hline & LAT (2.4-147.6) & 15 & 9.6 & 55 & $-2.0 \pm 0.3$ & $2.2 \pm 0.8$ & $0.4 \pm 0.3$ & $\cdots$ \\
\hline $150513 \mathrm{~A}$ & GBM $(-157.2-1.8)$ & 11 & 10.3 & 24 & $-2.5 \pm 0.4$ & $1.7 \pm 0.6$ & $0.12 \pm 0.07$ & $\cdots$ \\
\hline \multirow[t]{3}{*}{$150514 \mathrm{~A}$} & LTF $(0.0-4000.0)$ & 29 & 3.0 & 31 & $-1.3 \pm 0.5$ & $0.06 \pm 0.05$ & $2 \pm 3$ & $0.5 \pm 0.4$ \\
\hline & EXT (10.8-597.6) & 24 & 2.4 & 31 & $-1.1 \pm 0.4$ & $0.06 \pm 0.05$ & $0.5 \pm 0.6$ & $0.08 \pm 0.06$ \\
\hline & LAT (442.4-597.6) & 4 & 2.0 & 39 & $-1.0 \pm 0.4$ & $0.2 \pm 0.1$ & $0.7 \pm 0.7$ & $0.06 \pm 0.05$ \\
\hline \multirow[t]{4}{*}{$150523 \mathrm{~A}$} & GBM (1.8-84.2) & 40 & 27.9 & 178 & $-2.1 \pm 0.2$ & $6 \pm 1$ & $0.4 \pm 0.2$ & $\cdots$ \\
\hline & LTF $(0.0-500.0)$ & 106 & 51.0 & 248 & $-2.0 \pm 0.1$ & $1.6 \pm 0.3$ & $0.9 \pm 0.3$ & $\ldots$ \\
\hline & EXT (84.2-6129.1) & 344 & 31.3 & 116 & $-1.8 \pm 0.2$ & $0.20 \pm 0.05$ & $3 \pm 1$ & $\ldots$ \\
\hline & LAT (3.9-6129.1) & 384 & 59.3 & 211 & $-1.9 \pm 0.1$ & $0.39 \pm 0.06$ & $3 \pm 1$ & $\ldots$ \\
\hline \multirow[t]{4}{*}{$150627 \mathrm{~A}$} & LTF (0.0-4000.0) & 405 & 47.5 & 238 & $-1.7 \pm 0.1$ & $0.25 \pm 0.05$ & $3 \pm 1$ & $\cdots$ \\
\hline & EXT (69.9-6143.0) & 449 & 49.8 & 237 & $-1.7 \pm 0.1$ & $0.24 \pm 0.04$ & $4 \pm 1$ & $\cdots$ \\
\hline & LAT & 442 & 49.7 & 239 & $-1.7 \pm 0.1$ & $0.24 \pm 0.04$ & $4 \pm 1$ & $\ldots$ \\
\hline & $(152.0-6143.0)$ & & & & & & & \\
\hline \multirow[t]{4}{*}{$150702 \mathrm{~A}$} & LTF (0.0-4000.0) & 57 & 13.4 & 32 & $-2.2 \pm 0.3$ & $0.12 \pm 0.04$ & $0.3 \pm 0.2$ & $\ldots$ \\
\hline & EXT (47.2-1737.1) & 32 & 10.4 & 30 & $-2.3 \pm 0.4$ & $0.16 \pm 0.06$ & $0.15 \pm 0.09$ & $\cdots$ \\
\hline & LAT & 29 & 8.1 & 24 & $-2.2 \pm 0.4$ & $0.13 \pm 0.06$ & $0.11 \pm 0.08$ & $\cdots$ \\
\hline & $(512.6-1737.1)$ & & & & & & & \\
\hline \multirow[t]{4}{*}{$150902 \mathrm{~A}$} & GBM (3.8-17.4) & 21 & 20.4 & 160 & $-3.1 \pm 0.4$ & $37 \pm 8$ & $0.15 \pm 0.05$ & $\cdots$ \\
\hline & LTF $(0.0-500.0)$ & 110 & 51.1 & 203 & $-2.3 \pm 0.2$ & $2.0 \pm 0.4$ & $0.6 \pm 0.2$ & $\ldots$ \\
\hline & EXT (17.4-408.7) & 73 & 30.5 & 115 & $-2.1 \pm 0.2$ & $1.5 \pm 0.3$ & $0.6 \pm 0.2$ & $\ldots$ \\
\hline & LAT (3.5-408.7) & 94 & 49.3 & 212 & $-2.3 \pm 0.2$ & $2.4 \pm 0.4$ & $0.6 \pm 0.2$ & $\cdots$ \\
\hline $160310 \mathrm{~A}$ & LTF $(0.0-10000.0)$ & 71 & 6.4 & 30 & $-1.6 \pm 0.4$ & $0.10 \pm 0.08$ & $3 \pm 4$ & $\cdots$ \\
\hline 160314B & LTF $(0.0-10000.0)$ & 32 & 11.6 & 28 & $-2.1 \pm 0.3$ & $0.15 \pm 0.06$ & $1.2 \pm 0.8$ & $\cdots$ \\
\hline \multirow[t]{3}{*}{$160325 \mathrm{~A}$} & LTF (0.0-4000.0) & 73 & 36.2 & 97 & $-2.4 \pm 0.2$ & $0.46 \pm 0.10$ & $1.0 \pm 0.3$ & $\cdots$ \\
\hline & EXT (45.0-1555.7) & 69 & 34.2 & 93 & $-2.4 \pm 0.2$ & $0.5 \pm 0.1$ & $0.4 \pm 0.1$ & $\ldots$ \\
\hline & LAT (5.0-1555.7) & 71 & 35.4 & 95 & $-2.4 \pm 0.2$ & $0.48 \pm 0.10$ & $0.4 \pm 0.1$ & $\cdots$ \\
\hline \multirow[t]{2}{*}{$160422 \mathrm{~A}$} & LTF (0.0-4000.0) & 60 & 4.8 & 36 & $-1.3 \pm 0.3$ & $0.03 \pm 0.02$ & $1 \pm 1$ & $\cdots$ \\
\hline & EXT (13.1-1061.4) & 18 & 3.5 & 36 & $-1.2 \pm 0.3$ & $0.09 \pm 0.06$ & $1 \pm 1$ & $\cdots$ \\
\hline \multirow[t]{5}{*}{$160503 \mathrm{~A}$} & LTF (0.0-10000.0) & 54 & 16.8 & 33 & $-2.6 \pm 0.4$ & $0.17 \pm 0.05$ & $0.7 \pm 0.3$ & $\cdots$ \\
\hline & EXT & 141 & 28.3 & 37 & $-2.8 \pm 0.4$ & $0.12 \pm 0.03$ & $1.0 \pm 0.3$ & $\cdots$ \\
\hline & $(26.1-23075.4)$ & & & & & & & \\
\hline & LAT & 94 & 19.3 & 23 & $-3.1 \pm 0.5$ & $0.14 \pm 0.04$ & $0.7 \pm 0.2$ & $\cdots$ \\
\hline & $(5324.7-23075.4)$ & & & & & & & \\
\hline \multirow[t]{4}{*}{$160509 \mathrm{~A}$} & GBM (8.2-377.9) & 165 & 130.1 & 694 & $-2.4 \pm 0.1$ & $5.9 \pm 0.6$ & $1.1 \pm 0.2$ & $5.2 \pm 0.5$ \\
\hline & $\operatorname{LTF}(0.0-100.0)$ & 135 & 118.3 & 716 & $-2.9 \pm 0.2$ & $22 \pm 2$ & $0.7 \pm 0.1$ & $5.4 \pm 0.6$ \\
\hline & $\begin{array}{r}\text { EXT } \\
(377.9-5687.4)\end{array}$ & 183 & 19.0 & 39 & $-2.1 \pm 0.3$ & $0.11 \pm 0.04$ & $0.5 \pm 0.3$ & $1.5 \pm 0.5$ \\
\hline & LAT (9.6-5687.4) & 348 & 148.4 & 371 & $-2.4 \pm 0.1$ & $0.76 \pm 0.08$ & $2.3 \pm 0.4$ & $10 \pm 1$ \\
\hline \multirow[t]{4}{*}{$160521 \mathrm{~B}$} & LTF (0.0-4000.0) & 111 & 10.0 & 59 & $-1.5 \pm 0.2$ & $0.05 \pm 0.02$ & $1.1 \pm 0.8$ & $\cdots$ \\
\hline & EXT (3.1-11911.6) & 207 & 9.3 & 50 & $-1.4 \pm 0.3$ & $0.020 \pm 0.009$ & $2 \pm 1$ & $\cdots$ \\
\hline & LAT & 205 & 8.7 & 50 & $-1.4 \pm 0.3$ & $0.019 \pm 0.009$ & $2 \pm 1$ & $\cdots$ \\
\hline & (90.2-11911.6) & & & & & & & \\
\hline \multirow[t]{2}{*}{$160623 \mathrm{~A}$} & LTF (0.0-10000.0) & 401 & 97.0 & 258 & $-2.0 \pm 0.1$ & $0.8 \pm 0.1$ & $9 \pm 3$ & $2.1 \pm 0.4$ \\
\hline & $\begin{array}{r}\text { EXT } \\
(106.5-12160.0)\end{array}$ & 429 & 98.3 & 266 & $-2.0 \pm 0.1$ & $0.8 \pm 0.1$ & $11 \pm 3$ & $2.4 \pm 0.3$ \\
\hline
\end{tabular}


Table 4

(Continued)

\begin{tabular}{|c|c|c|c|c|c|c|c|c|}
\hline GRB Name & $\begin{array}{l}\text { Interval }\left(t_{0}-t_{1}\right) \\
\quad(\mathrm{s})\end{array}$ & $\begin{array}{l}\text { Trans. Ev. } \\
\text { in the ROI }\end{array}$ & $\begin{array}{l}\text { Trans. Ev. } \\
\text { Predicted }\end{array}$ & $\begin{array}{c}\text { Test } \\
\text { Statistic } \\
\text { (TS) }\end{array}$ & Spectral Index & $\begin{array}{c}\text { Flux } \\
\left(\times 10^{-5} \mathrm{~cm}^{-2} \mathrm{~s}^{-1}\right)\end{array}$ & $\begin{array}{c}\text { Fluence } \\
\left(\times 10^{-5} \mathrm{erg} \mathrm{cm}^{-2}\right)\end{array}$ & $\begin{array}{c}E_{\text {iso }}(100 \mathrm{MeV}- \\
10 \mathrm{GeV}) \\
\left(\times 10^{52} \mathrm{erg}\right)\end{array}$ \\
\hline & $\begin{array}{r}\text { LAT } \\
(401.5-12160.0)\end{array}$ & 427 & 98.3 & 267 & $-2.0 \pm 0.1$ & $0.8 \pm 0.1$ & $11 \pm 3$ & $2.4 \pm 0.3$ \\
\hline \multirow[t]{4}{*}{ 160625B } & GBM (188.5-641.8) & 272 & 258.3 & 2387 & $-2.33 \pm 0.08$ & $9.4 \pm 0.6$ & $2.5 \pm 0.3$ & $15 \pm 1$ \\
\hline & LTF $(0.0-500.0)$ & 270 & 255.8 & 2239 & $-2.43 \pm 0.08$ & $9.4 \pm 0.6$ & $2.4 \pm 0.3$ & $16 \pm 1$ \\
\hline & EXT (641.8-840.5) & 20 & 9.3 & 52 & $-1.8 \pm 0.3$ & $0.7 \pm 0.3$ & $0.3 \pm 0.3$ & $0.6 \pm 0.2$ \\
\hline & LAT (25.6-840.5) & 310 & 283.0 & 2222 & $-2.35 \pm 0.07$ & $6.0 \pm 0.4$ & $2.7 \pm 0.3$ & $17 \pm 1$ \\
\hline \multirow[t]{2}{*}{$160702 \mathrm{~A}$} & LTF $(0.0-4000.0)$ & 30 & 3.8 & 31 & $-1.4 \pm 0.4$ & $0.07 \pm 0.05$ & $2 \pm 2$ & $\ldots$ \\
\hline & EXT (0.2-2215.6) & 22 & 3.8 & 33 & $-1.4 \pm 0.4$ & $0.10 \pm 0.07$ & $2 \pm 2$ & $\cdots$ \\
\hline \multirow[t]{4}{*}{$160709 \mathrm{~A}$} & GBM (0.0-5.4) & 24 & 24.0 & 310 & $-2.4 \pm 0.3$ & $100 \pm 20$ & $0.3 \pm 0.1$ & $\cdots$ \\
\hline & LTF $(0.0-10.0)$ & 25 & 24.9 & 291 & $-2.5 \pm 0.3$ & $60 \pm 10$ & $0.3 \pm 0.1$ & $\cdots$ \\
\hline & EXT (5.4-25.8) & 5 & 3.9 & 29 & $-2.5 \pm 0.7$ & $5 \pm 2$ & $0.04 \pm 0.04$ & $\ldots$ \\
\hline & LAT (0.5-25.8) & 27 & 26.2 & 285 & $-2.4 \pm 0.3$ & $24 \pm 5$ & $0.3 \pm 0.1$ & $\cdots$ \\
\hline \multirow[t]{4}{*}{$160816 A$} & GBM (0.4-11.5) & 19 & 16.7 & 146 & $-2.7 \pm 0.4$ & $26 \pm 7$ & $0.11 \pm 0.04$ & $\cdots$ \\
\hline & LTF $(0.0-100.0)$ & 52 & 41.2 & 261 & $-2.3 \pm 0.2$ & $7 \pm 1$ & $0.4 \pm 0.1$ & $\ldots$ \\
\hline & EXT (11.5-1094.8) & 159 & 45.0 & 127 & $-2.2 \pm 0.2$ & $0.6 \pm 0.1$ & $0.5 \pm 0.2$ & $\ldots$ \\
\hline & LAT (1.1-1094.8) & 178 & 60.7 & 182 & $-2.2 \pm 0.2$ & $0.8 \pm 0.1$ & $0.6 \pm 0.2$ & $\cdots$ \\
\hline \multirow[t]{5}{*}{$160821 \mathrm{~A}$} & GBM (118.5-161.5) & 36 & 35.5 & 338 & $-5.1 \pm 0.7$ & $17 \pm 3$ & $0.16 \pm 0.03$ & $\cdots$ \\
\hline & LTF $(0.0-500.0)$ & 58 & 55.6 & 474 & $-2.7 \pm 0.2$ & $4.1 \pm 0.6$ & $0.8 \pm 0.2$ & $\cdots$ \\
\hline & EXT & 20 & 19.5 & 163 & $-1.8 \pm 0.2$ & $1.8 \pm 0.4$ & $5 \pm 3$ & $\cdots$ \\
\hline & $(161.5-1459.2)$ & & & & & & & \\
\hline & LAT (92.1-1459.2) & 57 & 55.6 & 516 & $-2.6 \pm 0.2$ & $4.2 \pm 0.6$ & $2.4 \pm 0.5$ & $\cdots$ \\
\hline \multirow[t]{3}{*}{$160829 \mathrm{~A}$} & LTF $(0.0-100.0)$ & 20 & 4.3 & 31 & $-1.3 \pm 0.4$ & $0.6 \pm 0.3$ & $0.5 \pm 0.7$ & $\ldots$ \\
\hline & EXT (0.4-31.7) & 5 & 2.8 & 31 & $-1.3 \pm 0.3$ & $1.2 \pm 0.8$ & $0.4 \pm 0.4$ & $\cdots$ \\
\hline & LAT (0.9-31.7) & 5 & 2.8 & 31 & $-1.3 \pm 0.3$ & $1.2 \pm 0.8$ & $0.4 \pm 0.4$ & $\cdots$ \\
\hline \multirow[t]{4}{*}{$160905 \mathrm{~A}$} & GBM (3.8-37.4) & 6 & 5.8 & 54 & $-1.7 \pm 0.3$ & $3 \pm 1$ & $0.3 \pm 0.3$ & $\cdots$ \\
\hline & LTF $(0.0-500.0)$ & 62 & 28.1 & 147 & $-1.8 \pm 0.2$ & $0.9 \pm 0.2$ & $0.8 \pm 0.4$ & $\ldots$ \\
\hline & EXT (37.4-463.3) & 52 & 22.2 & 106 & $-1.8 \pm 0.2$ & $0.8 \pm 0.2$ & $0.6 \pm 0.3$ & $\ldots$ \\
\hline & LAT (7.8-463.3) & 57 & 27.3 & 147 & $-1.8 \pm 0.2$ & $0.9 \pm 0.2$ & $0.8 \pm 0.4$ & $\cdots$ \\
\hline 160910A & LTF $(0.0-500.0)$ & 14 & 9.0 & 44 & $-2.5 \pm 0.5$ & $1.6 \pm 0.6$ & $0.4 \pm 0.2$ & $\cdots$ \\
\hline \multirow[t]{3}{*}{$161015 \mathrm{~A}$} & GBM (0.3-15.4) & 12 & 10.8 & 95 & $-2.2 \pm 0.4$ & $16 \pm 5$ & $0.2 \pm 0.1$ & $\cdots$ \\
\hline & LTF $(0.0-10.0)$ & 12 & 11.0 & 104 & $-2.2 \pm 0.3$ & $24 \pm 8$ & $0.2 \pm 0.1$ & $\cdots$ \\
\hline & LAT (1.1-7.5) & 9 & 8.1 & 68 & $-2.7 \pm 0.6$ & $30 \pm 10$ & $0.07 \pm 0.04$ & $\cdots$ \\
\hline \multirow[t]{3}{*}{$161109 \mathrm{~A}$} & LTF $(0.0-4000.0)$ & 51 & 18.3 & 44 & $-2.2 \pm 0.3$ & $0.31 \pm 0.10$ & $0.9 \pm 0.4$ & $\cdots$ \\
\hline & EXT (27.4-890.9) & 15 & 11.7 & 47 & $-2.2 \pm 0.3$ & $0.8 \pm 0.3$ & $0.5 \pm 0.3$ & $\ldots$ \\
\hline & LAT (423.4-890.9) & 14 & 10.6 & 43 & $-2.2 \pm 0.3$ & $0.8 \pm 0.3$ & $0.3 \pm 0.2$ & $\cdots$ \\
\hline \multirow[t]{4}{*}{ 170115B } & GBM (0.5-44.8) & 22 & 17.3 & 92 & $-3.2 \pm 0.5$ & $7 \pm 2$ & $0.09 \pm 0.03$ & $\cdots$ \\
\hline & LTF $(0.0-10.0)$ & 15 & 13.5 & 111 & $-3.0 \pm 0.5$ & $24 \pm 7$ & $0.08 \pm 0.03$ & $\cdots$ \\
\hline & EXT (44.8-1027.9) & 105 & 24.8 & 40 & $-2.5 \pm 0.3$ & $0.4 \pm 0.1$ & $0.17 \pm 0.07$ & $\cdots$ \\
\hline & LAT (1.3-1027.9) & 127 & 40.5 & 69 & $-2.7 \pm 0.4$ & $0.6 \pm 0.2$ & $0.24 \pm 0.05$ & $\cdots$ \\
\hline \multirow[t]{3}{*}{$170127 \mathrm{C}$} & LTF $(0.0-4000.0)$ & 65 & 15.6 & 32 & $-3.1 \pm 0.5$ & $0.12 \pm 0.04$ & $0.14 \pm 0.05$ & $\cdots$ \\
\hline & EXT (0.1-2889.0) & 63 & 15.7 & 33 & $-3.1 \pm 0.5$ & $0.12 \pm 0.04$ & $0.10 \pm 0.04$ & $\cdots$ \\
\hline & $\begin{array}{r}\text { LAT } \\
(664.8-2889.0)\end{array}$ & 52 & 14.5 & 36 & $-2.9 \pm 0.5$ & $0.12 \pm 0.04$ & $0.09 \pm 0.03$ & $\cdots$ \\
\hline \multirow[t]{4}{*}{$170214 \mathrm{~A}$} & GBM (12.5-135.4) & 123 & 114.2 & 846 & $-2.7 \pm 0.1$ & $19 \pm 2$ & $0.9 \pm 0.1$ & $32 \pm 5$ \\
\hline & LTF $(0.0-500.0)$ & 265 & 228.7 & 1493 & $-2.45 \pm 0.09$ & $8.2 \pm 0.6$ & $2.0 \pm 0.2$ & $49 \pm 4$ \\
\hline & EXT (135.4-752.0) & 179 & 138.8 & 819 & $-2.3 \pm 0.1$ & $3.8 \pm 0.4$ & $1.4 \pm 0.3$ & $27 \pm 2$ \\
\hline & LAT (39.5-752.0) & 297 & 250.3 & 1571 & $-2.45 \pm 0.09$ & $6.1 \pm 0.4$ & $2.1 \pm 0.2$ & $53 \pm 4$ \\
\hline \multirow[t]{3}{*}{ 170306B } & LTF $(0.0-100.0)$ & 6 & 5.9 & 35 & $-2.7 \pm 0.6$ & $2.2 \pm 0.9$ & $0.09 \pm 0.05$ & $\ldots$ \\
\hline & EXT (23.6-47.4) & 3 & 3.0 & 28 & $-2.8 \pm 0.9$ & $5 \pm 3$ & $0.04 \pm 0.03$ & $\cdots$ \\
\hline & LAT (21.2-47.4) & 3 & 3.0 & 27 & $-2.8 \pm 0.9$ & $4 \pm 2$ & $0.04 \pm 0.03$ & $\cdots$ \\
\hline \multirow[t]{3}{*}{$170329 \mathrm{~A}$} & GBM (0.3-33.8) & 11 & 9.8 & 91 & $-2.1 \pm 0.4$ & $6 \pm 2$ & $0.2 \pm 0.1$ & $\cdots$ \\
\hline & LTF $(0.0-100.0)$ & 19 & 14.7 & 80 & $-2.4 \pm 0.3$ & $3.2 \pm 0.9$ & $0.18 \pm 0.09$ & $\cdots$ \\
\hline & LAT (3.9-52.4) & 14 & 11.3 & 69 & $-2.2 \pm 0.3$ & $5 \pm 2$ & $0.2 \pm 0.1$ & $\cdots$ \\
\hline
\end{tabular}


Table 4

(Continued)

\begin{tabular}{|c|c|c|c|c|c|c|c|c|}
\hline GRB Name & $\begin{array}{l}\text { Interval }\left(t_{0}-t_{1}\right) \\
\quad(\mathrm{s})\end{array}$ & $\begin{array}{l}\text { Trans. Ev. } \\
\text { in the ROI }\end{array}$ & $\begin{array}{l}\text { Trans. Ev. } \\
\text { Predicted }\end{array}$ & $\begin{array}{c}\text { Test } \\
\text { Statistic } \\
\text { (TS) }\end{array}$ & Spectral Index & $\begin{array}{c}\text { Flux } \\
\left(\times 10^{-5} \mathrm{~cm}^{-2} \mathrm{~s}^{-1}\right)\end{array}$ & $\begin{array}{c}\text { Fluence } \\
\left(\times 10^{-5} \mathrm{erg} \mathrm{cm}^{-2}\right)\end{array}$ & $\begin{array}{c}E_{\text {iso }}(100 \mathrm{MeV}- \\
10 \mathrm{GeV}) \\
\left(\times 10^{52} \mathrm{erg}\right)\end{array}$ \\
\hline \multirow[t]{4}{*}{$170405 \mathrm{~A}$} & GBM (7.4-86.0) & 10 & 8.4 & 33 & $-5 \pm 2$ & $4 \pm 2$ & $0.07 \pm 0.03$ & $300 \pm 800$ \\
\hline & LTF (0.0-4000.0) & 73 & 29.1 & 59 & $-2.7 \pm 0.3$ & $0.4 \pm 0.1$ & $0.7 \pm 0.2$ & $50 \pm 20$ \\
\hline & EXT (86.0-868.0) & 45 & 19.8 & 50 & $-2.5 \pm 0.3$ & $0.5 \pm 0.1$ & $0.17 \pm 0.07$ & $9 \pm 4$ \\
\hline & LAT (17.8-868.0) & 55 & 26.1 & 56 & $-2.8 \pm 0.3$ & $0.6 \pm 0.2$ & $0.19 \pm 0.05$ & $16 \pm 7$ \\
\hline \multirow[t]{3}{*}{ 170409A } & LTF $(0.0-500.0)$ & 18 & 13.5 & 55 & $-2.1 \pm 0.3$ & $1.2 \pm 0.4$ & $0.6 \pm 0.4$ & $\cdots$ \\
\hline & EXT (93.4-440.3) & 18 & 13.5 & 55 & $-2.1 \pm 0.3$ & $1.3 \pm 0.4$ & $0.4 \pm 0.3$ & $\cdots$ \\
\hline & LAT (178.4-440.3) & 18 & 13.4 & 54 & $-2.1 \pm 0.3$ & $1.3 \pm 0.4$ & $0.3 \pm 0.2$ & $\cdots$ \\
\hline \multirow[t]{4}{*}{$170424 \mathrm{~A}$} & GBM (2.8-56.1) & 3 & 3.0 & 31 & $-1.9 \pm 0.5$ & $2 \pm 1$ & $0.1 \pm 0.2$ & $\cdots$ \\
\hline & LTF $(0.0-500.0)$ & 18 & 12.8 & 44 & $-2.3 \pm 0.3$ & $1.1 \pm 0.3$ & $0.3 \pm 0.2$ & $\cdots$ \\
\hline & EXT (56.1-107.7) & 3 & 3.0 & 25 & $-2.4 \pm 0.7$ & $2 \pm 1$ & $0.06 \pm 0.06$ & $\cdots$ \\
\hline & LAT (21.8-107.7) & 5 & 5.0 & 36 & $-2.0 \pm 0.4$ & $1.9 \pm 0.9$ & $0.2 \pm 0.2$ & $\cdots$ \\
\hline \multirow[t]{3}{*}{$170510 \mathrm{~A}$} & GBM (2.8-130.6) & 9 & 6.9 & 42 & $-2.2 \pm 0.4$ & $3 \pm 1$ & $0.3 \pm 0.2$ & $\cdots$ \\
\hline & LTF (0.0-100.0) & 8 & 6.6 & 47 & $-2.2 \pm 0.4$ & $4 \pm 2$ & $0.3 \pm 0.3$ & $\cdots$ \\
\hline & LAT (26.8-154.2) & 10 & 9.0 & 36 & $-2.3 \pm 0.4$ & $4 \pm 1$ & $0.3 \pm 0.2$ & $\cdots$ \\
\hline \multirow[t]{4}{*}{$170522 \mathrm{~A}$} & GBM (0.6-8.0) & 6 & 5.9 & 71 & $-1.8 \pm 0.3$ & $17 \pm 7$ & $0.2 \pm 0.3$ & $\cdots$ \\
\hline & LTF $(0.0-100.0)$ & 17 & 13.0 & 98 & $-1.7 \pm 0.2$ & $2.9 \pm 0.9$ & $0.7 \pm 0.5$ & $\cdots$ \\
\hline & EXT (8.0-41.6) & 5 & 4.9 & 44 & $-1.5 \pm 0.3$ & $3 \pm 1$ & $0.5 \pm 0.5$ & $\cdots$ \\
\hline & LAT (2.8-41.6) & 10 & 9.7 & 104 & $-1.6 \pm 0.2$ & $5 \pm 2$ & $0.8 \pm 0.6$ & $\cdots$ \\
\hline \multirow[t]{2}{*}{ 170728B } & GBM (0.0-46.3) & 5 & 4.8 & 21 & $-2.2 \pm 0.5$ & $2.0 \pm 1.0$ & $0.06 \pm 0.06$ & $\cdots$ \\
\hline & LTF $(0.0-500.0)$ & 18 & 11.0 & 30 & $-2.5 \pm 0.4$ & $0.4 \pm 0.2$ & $0.09 \pm 0.05$ & $\cdots$ \\
\hline \multirow[t]{3}{*}{ 170808B } & LTF (0.0-4000.0) & 52 & 25.1 & 66 & $-2.3 \pm 0.3$ & $0.5 \pm 0.1$ & $1.2 \pm 0.5$ & $\cdots$ \\
\hline & EXT (21.8-6205.9) & 97 & 24.0 & 52 & $-2.1 \pm 0.3$ & $0.15 \pm 0.04$ & $0.7 \pm 0.4$ & $\cdots$ \\
\hline & LAT (13.7-6205.9) & 99 & 26.8 & 55 & $-2.2 \pm 0.3$ & $0.17 \pm 0.05$ & $0.7 \pm 0.3$ & $\cdots$ \\
\hline $170825 B$ & LTF $(0.0-10.0)$ & 3 & 3.0 & 25 & $-3 \pm 1$ & $10 \pm 6$ & $0.03 \pm 0.02$ & $\cdots$ \\
\hline \multirow[t]{4}{*}{ 170906A } & LTF (0.0-10000.0) & 197 & 58.5 & 204 & $-2.1 \pm 0.1$ & $0.41 \pm 0.07$ & $4 \pm 1$ & $\cdots$ \\
\hline & EXT (90.9-1894.3) & 151 & 55.5 & 205 & $-2.1 \pm 0.1$ & $0.57 \pm 0.09$ & $1.0 \pm 0.3$ & $\cdots$ \\
\hline & LAT & 149 & 55.1 & 206 & $-2.1 \pm 0.1$ & $0.57 \pm 0.09$ & $1.0 \pm 0.3$ & $\cdots$ \\
\hline & $(162.1-1894.3)$ & & & & & & & \\
\hline \multirow[t]{2}{*}{ 170921B } & LTF $(0.0-10000.0)$ & 55 & 9.0 & 28 & $-1.9 \pm 0.4$ & $0.07 \pm 0.03$ & $0.9 \pm 0.8$ & $\cdots$ \\
\hline & EXT (40.4-1058.6) & 15 & 4.6 & 26 & $-1.7 \pm 0.4$ & $0.12 \pm 0.07$ & $0.3 \pm 0.4$ & $\cdots$ \\
\hline \multirow[t]{3}{*}{$171010 \mathrm{~A}$} & LTF (0.0-10000.0) & 231 & 77.6 & 234 & $-2.0 \pm 0.1$ & $0.22 \pm 0.03$ & $2.2 \pm 0.6$ & $0.43 \pm 0.07$ \\
\hline & $\begin{array}{r}\text { EXT } \\
(123.9-2984.8)\end{array}$ & 141 & 69.0 & 235 & $-2.0 \pm 0.1$ & $0.41 \pm 0.06$ & $1.2 \pm 0.3$ & $0.23 \pm 0.04$ \\
\hline & $\begin{array}{r}\text { LAT } \\
(335.6-2984.8)\end{array}$ & 141 & 69.0 & 235 & $-2.0 \pm 0.1$ & $0.41 \pm 0.06$ & $1.1 \pm 0.3$ & $0.21 \pm 0.03$ \\
\hline $171011 \mathrm{C}$ & GBM $(-0.4-0.0)$ & 3 & 3.0 & 52 & $-2.2 \pm 0.7$ & $120 \pm 70$ & $0.04 \pm 0.05$ & $\cdots$ \\
\hline \multirow[t]{3}{*}{$171102 \mathrm{~A}$} & LTF (0.0-500.0) & 14 & 10.5 & 38 & $-2.4 \pm 0.4$ & $0.4 \pm 0.1$ & $0.10 \pm 0.06$ & $\cdots$ \\
\hline & EXT (56.1-349.9) & 7 & 5.3 & 22 & $-2.4 \pm 0.6$ & $0.3 \pm 0.2$ & $0.05 \pm 0.04$ & $\cdots$ \\
\hline & LAT (34.9-349.9) & 8 & 6.3 & 25 & $-2.6 \pm 0.6$ & $0.4 \pm 0.2$ & $0.05 \pm 0.03$ & $\cdots$ \\
\hline \multirow[t]{4}{*}{$171120 \mathrm{~A}$} & GBM (0.0-44.1) & 6 & 5.9 & 66 & $-2.1 \pm 0.4$ & $2 \pm 1$ & $0.10 \pm 0.10$ & $\cdots$ \\
\hline & LTF (0.0-4000.0) & 50 & 36.4 & 147 & $-2.3 \pm 0.2$ & $0.8 \pm 0.1$ & $1.8 \pm 0.6$ & $\cdots$ \\
\hline & EXT (44.1-5276.0) & 74 & 33.1 & 104 & $-2.3 \pm 0.2$ & $0.34 \pm 0.07$ & $1.2 \pm 0.4$ & $\cdots$ \\
\hline & LAT (0.3-5276.0) & 79 & 37.4 & 134 & $-2.2 \pm 0.2$ & $0.37 \pm 0.07$ & $1.5 \pm 0.5$ & $\cdots$ \\
\hline \multirow[t]{3}{*}{$171124 \mathrm{~A}$} & GBM $(-0.7-25.5)$ & 22 & 21.0 & 192 & $-2.0 \pm 0.2$ & $12 \pm 3$ & $0.3 \pm 0.2$ & $\cdots$ \\
\hline & LTF (0.0-100.0) & 33 & 23.6 & 125 & $-2.1 \pm 0.2$ & $3.5 \pm 0.8$ & $0.3 \pm 0.1$ & $\cdots$ \\
\hline & LAT (3.4-321.3) & 45 & 26.2 & 90 & $-2.2 \pm 0.2$ & $1.3 \pm 0.3$ & $0.3 \pm 0.1$ & $\cdots$ \\
\hline \multirow[t]{3}{*}{$171210 \mathrm{~A}$} & LTF $(0.0-10000.0)$ & 169 & 28.2 & 65 & $-2.2 \pm 0.2$ & $0.11 \pm 0.03$ & $0.8 \pm 0.3$ & $\ldots$ \\
\hline & $\begin{array}{r}\text { EXT } \\
(146.7-1374.5)\end{array}$ & 54 & 19.9 & 45 & $-2.4 \pm 0.3$ & $0.27 \pm 0.07$ & $0.16 \pm 0.07$ & $\cdots$ \\
\hline & LAT (5.9-1374.5) & 64 & 24.1 & 59 & $-2.4 \pm 0.3$ & $0.30 \pm 0.08$ & $0.22 \pm 0.09$ & $\cdots$ \\
\hline 171212B & LTF (0.0-4000.0) & 26 & 7.7 & 26 & $-2.3 \pm 0.5$ & $0.18 \pm 0.08$ & $0.5 \pm 0.3$ & $\cdots$ \\
\hline
\end{tabular}


Table 4

(Continued)

\begin{tabular}{|c|c|c|c|c|c|c|c|c|}
\hline GRB Name & $\begin{array}{l}\text { Interval }\left(t_{0}-t_{1}\right) \\
\quad(\mathrm{s})\end{array}$ & $\begin{array}{l}\text { Trans. Ev. } \\
\text { in the ROI }\end{array}$ & $\begin{array}{l}\text { Trans. Ev. } \\
\text { Predicted }\end{array}$ & $\begin{array}{l}\text { Test } \\
\text { Statistic } \\
\text { (TS) }\end{array}$ & Spectral Index & $\begin{array}{c}\text { Flux } \\
\left(\times 10^{-5} \mathrm{~cm}^{-2} \mathrm{~s}^{-1}\right)\end{array}$ & $\begin{array}{c}\text { Fluence } \\
\left(\times 10^{-5} \mathrm{erg} \mathrm{cm}^{-2}\right)\end{array}$ & $\begin{array}{c}E_{\text {iso }}(100 \mathrm{MeV}- \\
10 \mathrm{GeV}) \\
\left(\times 10^{52} \mathrm{erg}\right)\end{array}$ \\
\hline \multirow[t]{3}{*}{$180210 \mathrm{~A}$} & LTF $(0.0-4000.0)$ & 81 & 40.3 & 184 & $-1.8 \pm 0.1$ & $0.40 \pm 0.08$ & $3 \pm 1$ & $\ldots$ \\
\hline & EXT (43.0-1621.1) & 75 & 33.9 & 168 & $-1.7 \pm 0.1$ & $0.35 \pm 0.07$ & $1.3 \pm 0.5$ & $\cdots$ \\
\hline & LAT (23.1-1621.1) & 77 & 35.8 & 176 & $-1.8 \pm 0.1$ & $0.36 \pm 0.07$ & $1.3 \pm 0.5$ & $\cdots$ \\
\hline \multirow[t]{4}{*}{$180305 \mathrm{~A}$} & LTF $(0.0-4000.0)$ & 119 & 11.9 & 32 & $-1.8 \pm 0.3$ & $0.11 \pm 0.05$ & $0.8 \pm 0.6$ & $\cdots$ \\
\hline & EXT (14.6-2038.0) & 100 & 9.1 & 27 & $-1.7 \pm 0.3$ & $0.10 \pm 0.05$ & $0.5 \pm 0.4$ & $\cdots$ \\
\hline & LAT & 21 & 6.0 & 21 & $-2 \pm 1$ & $0.3 \pm 0.5$ & $0.2 \pm 0.2$ & $\ldots$ \\
\hline & $(1613.8-2038.0)$ & & & & & & & \\
\hline \multirow[t]{4}{*}{$180526 \mathrm{~A}$} & LTF $(0.0-10000.0)$ & 110 & 14.0 & 48 & $-1.8 \pm 0.3$ & $0.13 \pm 0.05$ & $3 \pm 2$ & $\cdots$ \\
\hline & EXT (87.0-1948.6) & 47 & 13.2 & 51 & $-1.8 \pm 0.3$ & $0.4 \pm 0.1$ & $1.2 \pm 0.8$ & $\cdots$ \\
\hline & LAT & 44 & 11.9 & 48 & $-1.8 \pm 0.3$ & $0.3 \pm 0.1$ & $0.6 \pm 0.5$ & $\cdots$ \\
\hline & (808.9-1948.6) & & & & & & & \\
\hline \multirow[t]{4}{*}{$180703 \mathrm{~A}$} & GBM (1.5-22.3) & 4 & 3.8 & 24 & $-3 \pm 1$ & $5 \pm 3$ & $0.03 \pm 0.02$ & $\cdots$ \\
\hline & LTF $(0.0-4000.0)$ & 40 & 21.9 & 54 & $-2.5 \pm 0.3$ & $0.24 \pm 0.07$ & $0.4 \pm 0.1$ & $\ldots$ \\
\hline & EXT (22.3-1614.0) & 31 & 16.7 & 43 & $-2.4 \pm 0.3$ & $0.27 \pm 0.09$ & $0.2 \pm 0.1$ & $\ldots$ \\
\hline & LAT (3.8-1614.0) & 35 & 19.3 & 47 & $-2.5 \pm 0.3$ & $0.31 \pm 0.09$ & $0.24 \pm 0.09$ & $\cdots$ \\
\hline \multirow[t]{3}{*}{ 180703B } & LTF $(0.0-100.0)$ & 6 & 5.0 & 36 & $-1.8 \pm 0.4$ & $0.8 \pm 0.3$ & $0.1 \pm 0.2$ & $\cdots$ \\
\hline & EXT (1.7-34.2) & 4 & 4.0 & 43 & $-1.6 \pm 0.4$ & $1.8 \pm 0.9$ & $0.2 \pm 0.2$ & $\cdots$ \\
\hline & LAT (8.0-34.2) & 3 & 3.0 & 27 & $-1.7 \pm 0.4$ & $2 \pm 1$ & $0.1 \pm 0.2$ & $\cdots$ \\
\hline \multirow[t]{3}{*}{ 180718B } & GBM (1.6-99.9) & 12 & 11.2 & 21 & $-2.8 \pm 0.5$ & $3.0 \pm 1.0$ & $0.11 \pm 0.05$ & $\cdots$ \\
\hline & LTF $(0.0-10.0)$ & 8 & 8.0 & 72 & $-2.9 \pm 0.6$ & $20 \pm 7$ & $0.07 \pm 0.03$ & $\cdots$ \\
\hline & LAT (1.1-16.2) & 8 & 7.9 & 60 & $-2.9 \pm 0.6$ & $13 \pm 5$ & $0.07 \pm 0.04$ & $\cdots$ \\
\hline \multirow[t]{4}{*}{ 180720B } & GBM (4.4-53.2) & 22 & 20.2 & 162 & $-3.2 \pm 0.4$ & $13 \pm 3$ & $0.19 \pm 0.05$ & $0.39 \pm 0.09$ \\
\hline & LTF $(0.0-500.0)$ & 152 & 136.8 & 984 & $-2.24 \pm 0.10$ & $6.0 \pm 0.6$ & $2.0 \pm 0.4$ & $2.1 \pm 0.2$ \\
\hline & EXT (53.2-625.0) & 136 & 120.9 & 871 & $-2.15 \pm 0.10$ & $4.6 \pm 0.5$ & $2.1 \pm 0.4$ & $2.0 \pm 0.2$ \\
\hline & LAT (11.8-625.0) & 157 & 139.3 & 975 & $-2.23 \pm 0.10$ & $5.0 \pm 0.5$ & $2.1 \pm 0.4$ & $2.2 \pm 0.2$ \\
\hline
\end{tabular}


Table 5

Temporally Extended High-energy Emission

\begin{tabular}{|c|c|c|c|c|c|c|c|c|}
\hline GRB Name & $\begin{array}{c}\text { Peak Flux } \\
\left(\times 10^{-5} \mathrm{~cm}^{-2}\right. \\
\left.\mathrm{s}^{-1}\right)\end{array}$ & $\begin{array}{l}\text { Peak Flux Time } \\
\text { (s) }\end{array}$ & $\begin{array}{c}\text { Decay } \\
\text { Index (SPL) }\end{array}$ & $\begin{array}{c}\text { Decay Index } \\
1 \text { (BPL) }\end{array}$ & $\begin{array}{l}\text { Decay Index } \\
2 \text { (BPL) }\end{array}$ & $\begin{array}{c}\text { Break } \\
\text { Time (BPL) }\end{array}$ & Decay Index & $\begin{array}{c}\text { Extended } \\
\text { Emission } \\
\text { Duration } \\
\text { (s) }\end{array}$ \\
\hline $080825 \mathrm{C}$ & $21 \pm 8$ & $10 \pm 20$ & $1.45 \pm 0.03$ & $\ldots$ & $\ldots$ & $\ldots$ & $1.45 \pm 0.03$ & 170.4 \\
\hline 080916C & $1800 \pm 700$ & $5.87 \pm 0.08$ & $1.1 \pm 0.1$ & $1.6 \pm 0.2$ & $0.4 \pm 0.3$ & $3.1 \pm 0.3$ & $0.4 \pm 0.3$ & 1528.8 \\
\hline 081009 & $1.9 \pm 1.0$ & $100 \pm 200$ & $0.9 \pm 0.2$ & $\ldots$ & $\ldots$ & $\ldots$ & $0.9 \pm 0.2$ & 1182.4 \\
\hline 090323 & $13 \pm 4$ & $90 \pm 30$ & $1.1 \pm 0.1$ & $1 \pm 2$ & $1.1 \pm 0.2$ & $2.4 \pm 0.4$ & $1.1 \pm 0.1$ & 5312.3 \\
\hline 090328 & $16 \pm 5$ & $20 \pm 50$ & $0.99 \pm 0.09$ & $0.7 \pm 0.4$ & $1.1 \pm 0.1$ & $2.4 \pm 0.5$ & $0.99 \pm 0.09$ & 6136.3 \\
\hline 090510 & $6000 \pm 3000$ & $0.85 \pm 0.01$ & $1.81 \pm 0.08$ & $2.3 \pm 0.2$ & $1.3 \pm 0.2$ & $0.6 \pm 0.2$ & $1.3 \pm 0.2$ & 169.9 \\
\hline 090626 & $4 \pm 1$ & $100 \pm 40$ & $0.9 \pm 0.2$ & $\ldots$ & $\ldots$ & $\ldots$ & $0.9 \pm 0.2$ & 548.3 \\
\hline 090902B & $1300 \pm 500$ & $8.0 \pm 0.1$ & $1.63 \pm 0.08$ & $1.9 \pm 0.2$ & $1.2 \pm 0.2$ & $2.2 \pm 0.3$ & $1.2 \pm 0.2$ & 883.7 \\
\hline 090926A & $7000 \pm 3000$ & $9.91 \pm 0.02$ & $1.39 \pm 0.08$ & $1.8 \pm 0.2$ & $1.1 \pm 0.2$ & $2.0 \pm 0.3$ & $1.1 \pm 0.2$ & 4417.3 \\
\hline 091003 & $4 \pm 2$ & $20 \pm 40$ & $0.9 \pm 0.2$ & $0.7 \pm 0.4$ & $2 \pm 1$ & $2.5 \pm 0.4$ & $0.9 \pm 0.2$ & 391.0 \\
\hline 091031 & $30 \pm 10$ & $1 \pm 3$ & $1.3 \pm 0.2$ & $\ldots$ & $\ldots$ & $\ldots$ & $1.3 \pm 0.2$ & 408.1 \\
\hline 091120 & $0.8 \pm 0.3$ & $200 \pm 300$ & $0.54 \pm 0.09$ & $\ldots$ & $\ldots$ & $\ldots$ & $0.54 \pm 0.09$ & 772.1 \\
\hline 100116A & $7 \pm 2$ & $110 \pm 20$ & $2.7 \pm 0.2$ & $\ldots$ & $\ldots$ & $\ldots$ & $2.7 \pm 0.2$ & 652.9 \\
\hline 100414A & $40 \pm 10$ & $30 \pm 30$ & $1.3 \pm 0.1$ & $1.8 \pm 0.2$ & $0.3 \pm 0.6$ & $2.9 \pm 0.3$ & $0.3 \pm 0.6$ & 5487.5 \\
\hline $100423 B$ & $0.3 \pm 0.4$ & $0 \pm 200$ & $0.2 \pm 0.1$ & $\ldots$ & $\ldots$ & $\ldots$ & $0.2 \pm 0.1$ & 13.9 \\
\hline $100511 \mathrm{~A}$ & $1.1 \pm 0.4$ & $100 \pm 200$ & $0.58 \pm 0.07$ & $\ldots$ & $\ldots$ & $\ldots$ & $0.58 \pm 0.07$ & 6326.6 \\
\hline $100728 \mathrm{~A}$ & $0.3 \pm 0.1$ & $300 \pm 600$ & $0.8 \pm 0.5$ & $\ldots$ & $\ldots$ & $\ldots$ & $0.8 \pm 0.5$ & 1091.8 \\
\hline 101014A & $0.05 \pm 0.03$ & $2500 \pm 1000$ & $-0.2 \pm 0.3$ & $\ldots$ & $\ldots$ & $\ldots$ & $-0.2 \pm 0.3$ & 1925.3 \\
\hline $110120 \mathrm{~A}$ & $1.7 \pm 0.7$ & $50 \pm 70$ & $0.6 \pm 0.4$ & $\ldots$ & $\ldots$ & $\ldots$ & $0.6 \pm 0.4$ & 1112.3 \\
\hline $110428 \mathrm{~A}$ & $9 \pm 5$ & $12 \pm 10$ & $1.0 \pm 0.1$ & $\ldots$ & $\ldots$ & $\ldots$ & $1.0 \pm 0.1$ & 386.2 \\
\hline $110518 \mathrm{~A}$ & $0.11 \pm 0.06$ & $2000 \pm 1000$ & $0.7 \pm 0.5$ & $\ldots$ & $\ldots$ & $\ldots$ & $0.7 \pm 0.5$ & 396.0 \\
\hline $110625 \mathrm{~A}$ & $8 \pm 4$ & $260 \pm 30$ & $0.6 \pm 0.3$ & $\ldots$ & $\ldots$ & $\ldots$ & $0.6 \pm 0.3$ & 371.4 \\
\hline $110721 \mathrm{~A}$ & $600 \pm 200$ & $0.1 \pm 0.2$ & $1.0 \pm 0.1$ & $\ldots$ & $\ldots$ & $\ldots$ & $1.0 \pm 0.1$ & 120.6 \\
\hline $110731 \mathrm{~A}$ & $1400 \pm 500$ & $5.63 \pm 0.08$ & $1.5 \pm 0.1$ & $1.8 \pm 0.1$ & $0 \pm 1$ & $2.4 \pm 0.5$ & $0 \pm 1$ & 434.8 \\
\hline 111210B & $1.0 \pm 0.4$ & $100 \pm 200$ & $0.5 \pm 0.2$ & $\ldots$ & $\ldots$ & $\ldots$ & $0.5 \pm 0.2$ & 387.5 \\
\hline $120226 \mathrm{~A}$ & $1.8 \pm 0.9$ & $30 \pm 60$ & $1.21 \pm 0.07$ & $\ldots$ & $\ldots$ & $\ldots$ & $1.21 \pm 0.07$ & 254.3 \\
\hline $120316 \mathrm{~A}$ & $2.0 \pm 0.8$ & $40 \pm 60$ & $0.9 \pm 0.2$ & $\ldots$ & $\ldots$ & $\ldots$ & $0.9 \pm 0.2$ & 530.0 \\
\hline $120526 \mathrm{~A}$ & $0.4 \pm 0.2$ & $900 \pm 500$ & $0.7 \pm 0.1$ & $\ldots$ & $\ldots$ & $\ldots$ & $0.7 \pm 0.1$ & 2614.1 \\
\hline $120624 B$ & $8 \pm 3$ & $380 \pm 20$ & $1.2 \pm 0.3$ & $\ldots$ & $\ldots$ & $\ldots$ & $1.2 \pm 0.3$ & 1030.2 \\
\hline 120709A & $60 \pm 30$ & $0 \pm 2$ & $0.7 \pm 0.1$ & $\ldots$ & $\ldots$ & $\ldots$ & $0.7 \pm 0.1$ & 695.8 \\
\hline $120711 \mathrm{~A}$ & $2.1 \pm 0.7$ & $400 \pm 200$ & $1.6 \pm 0.2$ & $\ldots$ & $\ldots$ & $\ldots$ & $1.6 \pm 0.2$ & 5038.3 \\
\hline 120911B & $500 \pm 200$ & $20 \pm 1$ & $1.3 \pm 0.2$ & $\ldots$ & $\ldots$ & $\ldots$ & $1.3 \pm 0.2$ & 208.5 \\
\hline $130325 \mathrm{~A}$ & $0.2 \pm 0.1$ & $200 \pm 200$ & $0.1 \pm 0.3$ & $\ldots$ & $\ldots$ & $\ldots$ & $0.1 \pm 0.3$ & 705.7 \\
\hline $130327 \mathrm{~B}$ & $17 \pm 6$ & $35 \pm 9$ & $1.6 \pm 0.2$ & $1.3 \pm 0.9$ & $1.7 \pm 0.3$ & $1.9 \pm 0.8$ & $1.6 \pm 0.2$ & 515.2 \\
\hline 130427A & $300 \pm 100$ & $16.5 \pm 0.6$ & $1.24 \pm 0.06$ & $0.8 \pm 0.2$ & $1.4 \pm 0.1$ & $2.7 \pm 0.2$ & $1.4 \pm 0.1$ & 34366.0 \\
\hline $130502 B$ & $40 \pm 10$ & $28 \pm 8$ & $1.44 \pm 0.06$ & $\ldots$ & $\ldots$ & $\ldots$ & $1.44 \pm 0.06$ & 1304.2 \\
\hline $130504 \mathrm{C}$ & $1.6 \pm 0.6$ & $100 \pm 200$ & $0.77 \pm 0.06$ & $0.7 \pm 0.1$ & $1.0 \pm 0.5$ & $2.4 \pm 0.4$ & $0.77 \pm 0.06$ & 547.7 \\
\hline $130518 \mathrm{~A}$ & $5 \pm 2$ & $40 \pm 60$ & $1.1 \pm 0.2$ & $\ldots$ & $\ldots$ & $\ldots$ & $1.1 \pm 0.2$ & 316.9 \\
\hline 130606B & $1.3 \pm 0.5$ & $200 \pm 200$ & $0.7 \pm 0.2$ & $\ldots$ & $\ldots$ & $\ldots$ & $0.7 \pm 0.2$ & 396.7 \\
\hline $130821 \mathrm{~A}$ & $2.2 \pm 0.8$ & $70 \pm 80$ & $1.0 \pm 0.1$ & $\ldots$ & $\ldots$ & $\ldots$ & $1.0 \pm 0.1$ & 6069.8 \\
\hline $130828 \mathrm{~A}$ & $70 \pm 30$ & $35 \pm 2$ & $1.0 \pm 0.4$ & $\ldots$ & $\ldots$ & $\ldots$ & $1.0 \pm 0.4$ & 599.8 \\
\hline $131014 \mathrm{~A}$ & $5000 \pm 2000$ & $2.0 \pm 0.2$ & $0.8 \pm 0.2$ & $\ldots$ & $\ldots$ & $\ldots$ & $0.8 \pm 0.2$ & 198.6 \\
\hline 131029A & $22 \pm 8$ & $50 \pm 20$ & $1.1 \pm 0.2$ & $\ldots$ & $\ldots$ & $\ldots$ & $1.1 \pm 0.2$ & 518.5 \\
\hline 131108A & $3000 \pm 1000$ & $0.13 \pm 0.03$ & $1.5 \pm 0.2$ & $1.9 \pm 0.5$ & $1.2 \pm 0.6$ & $1.8 \pm 0.6$ & $1.5 \pm 0.2$ & 678.1 \\
\hline 131209A & $2.6 \pm 1.0$ & $30 \pm 60$ & $0.8 \pm 0.2$ & $\ldots$ & $\ldots$ & $\ldots$ & $0.8 \pm 0.2$ & 360.5 \\
\hline $131231 \mathrm{~A}$ & $5 \pm 2$ & $30 \pm 40$ & $1.0 \pm 0.2$ & $\ldots$ & $\ldots$ & $\ldots$ & $1.0 \pm 0.2$ & 4801.1 \\
\hline $140102 \mathrm{~A}$ & $50 \pm 20$ & $4 \pm 2$ & $1.2 \pm 0.4$ & $\ldots$ & $\ldots$ & $\ldots$ & $1.2 \pm 0.4$ & 57.1 \\
\hline 140104B & $0.5 \pm 0.3$ & $900 \pm 200$ & $0.3 \pm 0.6$ & $\ldots$ & $\ldots$ & $\ldots$ & $0.3 \pm 0.6$ & 946.8 \\
\hline $140110 \mathrm{~A}$ & $130 \pm 50$ & $1.0 \pm 0.9$ & $0.97 \pm 0.02$ & $\ldots$ & $\ldots$ & $\ldots$ & $0.97 \pm 0.02$ & 158.8 \\
\hline 140206B & $15 \pm 5$ & $30 \pm 20$ & $0.3 \pm 0.3$ & $\ldots$ & $\ldots$ & $\ldots$ & $0.3 \pm 0.3$ & 8584.4 \\
\hline $140402 \mathrm{~A}$ & $50 \pm 20$ & $0 \pm 3$ & $0.87 \pm 0.06$ & $\ldots$ & $\ldots$ & $\ldots$ & $0.87 \pm 0.06$ & 71.5 \\
\hline $140523 \mathrm{~A}$ & $30 \pm 10$ & $8 \pm 8$ & $1.0 \pm 0.1$ & $\ldots$ & $\ldots$ & $\ldots$ & $1.0 \pm 0.1$ & 468.6 \\
\hline 140810A & $0.4 \pm 0.2$ & $500 \pm 200$ & $0.8 \pm 0.2$ & $\ldots$ & $\ldots$ & $\ldots$ & $0.8 \pm 0.2$ & 18366.7 \\
\hline $141028 \mathrm{~A}$ & $25 \pm 10$ & $20 \pm 6$ & $0.97 \pm 0.03$ & $\ldots$ & $\ldots$ & $\ldots$ & $0.97 \pm 0.03$ & 489.6 \\
\hline $141102 \mathrm{~A}$ & $11 \pm 6$ & $10 \pm 10$ & $1.0 \pm 0.2$ & $\ldots$ & $\ldots$ & $\ldots$ & $1.0 \pm 0.2$ & 31.0 \\
\hline $141207 \mathrm{~A}$ & $150 \pm 60$ & $8 \pm 1$ & $1.88 \pm 0.03$ & $\ldots$ & $\ldots$ & $\ldots$ & $1.88 \pm 0.03$ & 732.6 \\
\hline $141222 \mathrm{~A}$ & $6 \pm 3$ & $50 \pm 30$ & $1.3 \pm 0.4$ & $\ldots$ & $\ldots$ & $\ldots$ & $1.3 \pm 0.4$ & 406.3 \\
\hline $150314 \mathrm{~A}$ & $4 \pm 1$ & $30 \pm 80$ & $0.9 \pm 0.1$ & $\ldots$ & $\ldots$ & $\ldots$ & $0.9 \pm 0.1$ & 3064.2 \\
\hline $150523 \mathrm{~A}$ & $20 \pm 7$ & $15 \pm 7$ & $1.0 \pm 0.3$ & $\ldots$ & $\ldots$ & $\ldots$ & $1.0 \pm 0.3$ & 6125.2 \\
\hline $150627 \mathrm{~A}$ & $3 \pm 1$ & $200 \pm 200$ & $0.9 \pm 0.2$ & $3.0 \pm 0.2$ & $0.41 \pm 0.07$ & $2.62 \pm 0.03$ & $0.41 \pm 0.07$ & 5991.0 \\
\hline
\end{tabular}


Table 5

(Continued)

\begin{tabular}{|c|c|c|c|c|c|c|c|c|}
\hline GRB Name & $\begin{array}{c}\text { Peak Flux } \\
\left(\times 10^{-5} \mathrm{~cm}^{-2}\right. \\
\left.\mathrm{s}^{-1}\right)\end{array}$ & $\begin{array}{c}\text { Peak Flux Time } \\
\text { (s) }\end{array}$ & $\begin{array}{c}\text { Decay } \\
\text { Index (SPL) }\end{array}$ & $\begin{array}{c}\text { Decay Index } \\
1 \text { (BPL) }\end{array}$ & $\begin{array}{c}\text { Decay Index } \\
2 \text { (BPL) }\end{array}$ & $\begin{array}{c}\text { Break } \\
\text { Time (BPL) } \\
\text { (s) }\end{array}$ & Decay Index & $\begin{array}{c}\text { Extended } \\
\text { Emission } \\
\text { Duration } \\
\text { (s) }\end{array}$ \\
\hline $150702 \mathrm{~A}$ & $0.2 \pm 0.1$ & $800 \pm 600$ & $0.4 \pm 0.3$ & $\ldots$ & $\ldots$ & $\ldots$ & $0.4 \pm 0.3$ & 1224.5 \\
\hline $150902 \mathrm{~A}$ & $60 \pm 20$ & $5 \pm 3$ & $1.0 \pm 0.2$ & $\ldots$ & $\ldots$ & $\ldots$ & $1.0 \pm 0.2$ & 405.2 \\
\hline $160325 \mathrm{~A}$ & $1.7 \pm 0.6$ & $100 \pm 100$ & $0.74 \pm 0.10$ & $\ldots$ & $\ldots$ & $\ldots$ & $0.74 \pm 0.10$ & 1550.7 \\
\hline $160509 \mathrm{~A}$ & $1500 \pm 500$ & $17.5 \pm 0.1$ & $1.1 \pm 0.1$ & $0.9 \pm 0.3$ & $1.3 \pm 0.3$ & $2.8 \pm 0.5$ & $1.1 \pm 0.1$ & 5677.8 \\
\hline $160521 \mathrm{~B}$ & $1.5 \pm 0.8$ & $90 \pm 70$ & $1.3 \pm 0.2$ & $\ldots$ & $\ldots$ & $\ldots$ & $1.3 \pm 0.2$ & 11821.5 \\
\hline $160623 \mathrm{~A}$ & $8 \pm 4$ & $500 \pm 100$ & $1.25 \pm 0.09$ & $\ldots$ & $\ldots$ & $\ldots$ & $1.25 \pm 0.09$ & 11758.4 \\
\hline $160625 \mathrm{~B}$ & $400 \pm 200$ & $200.6 \pm 0.3$ & $2.2 \pm 0.3$ & $\ldots$ & $\ldots$ & $\ldots$ & $2.2 \pm 0.3$ & 814.9 \\
\hline $160816 \mathrm{~A}$ & $40 \pm 10$ & $2 \pm 3$ & $1.2 \pm 0.1$ & $1.5 \pm 0.3$ & $0.9 \pm 0.6$ & $2.1 \pm 0.8$ & $1.2 \pm 0.1$ & 1093.7 \\
\hline $160821 \mathrm{~A}$ & $300 \pm 100$ & $137.3 \pm 0.7$ & $1.15 \pm 0.10$ & $\ldots$ & $\ldots$ & $\ldots$ & $1.15 \pm 0.10$ & 1367.1 \\
\hline $160905 \mathrm{~A}$ & $4 \pm 2$ & $40 \pm 30$ & $1.2 \pm 0.3$ & $\ldots$ & $\ldots$ & $\ldots$ & $1.2 \pm 0.3$ & 455.5 \\
\hline 161109A & $1.9 \pm 0.8$ & $500 \pm 200$ & $1.3 \pm 0.5$ & $\ldots$ & $\ldots$ & $\ldots$ & $1.3 \pm 0.5$ & 467.5 \\
\hline 170115B & $80 \pm 30$ & $7 \pm 1$ & $1.2 \pm 0.2$ & $2 \pm 2$ & $0.9 \pm 0.7$ & $2.3 \pm 0.4$ & $1.2 \pm 0.2$ & 1026.6 \\
\hline $170214 \mathrm{~A}$ & $400 \pm 100$ & $63.0 \pm 0.4$ & $1.7 \pm 0.3$ & $2 \pm 2$ & $1.6 \pm 0.5$ & $2.3 \pm 0.5$ & $1.7 \pm 0.3$ & 712.5 \\
\hline $170405 \mathrm{~A}$ & $8 \pm 3$ & $30 \pm 30$ & $1.27 \pm 0.01$ & $\ldots$ & $\ldots$ & $\ldots$ & $1.27 \pm 0.01$ & 850.1 \\
\hline 170409A & $1.8 \pm 0.7$ & $300 \pm 200$ & $1.3 \pm 0.1$ & $\ldots$ & $\ldots$ & $\ldots$ & $1.3 \pm 0.1$ & 261.9 \\
\hline 170808B & $4 \pm 1$ & $100 \pm 100$ & $1.0 \pm 0.2$ & $\ldots$ & $\ldots$ & $\ldots$ & $1.0 \pm 0.2$ & 6192.2 \\
\hline $170906 \mathrm{~A}$ & $3 \pm 1$ & $200 \pm 100$ & $0.8 \pm 0.1$ & $\ldots$ & $\ldots$ & $\ldots$ & $0.8 \pm 0.1$ & 1732.2 \\
\hline $171010 \mathrm{~A}$ & $3 \pm 1$ & $380 \pm 70$ & $1.3 \pm 0.2$ & $2.2 \pm 0.7$ & $1.0 \pm 0.3$ & $2.9 \pm 0.2$ & $1.0 \pm 0.3$ & 2649.2 \\
\hline $171102 \mathrm{~A}$ & $1.4 \pm 0.7$ & $60 \pm 90$ & $1.02 \pm 0.07$ & $\ldots$ & $\ldots$ & $\ldots$ & $1.02 \pm 0.07$ & 315.0 \\
\hline $171120 \mathrm{~A}$ & $2 \pm 1$ & $10 \pm 40$ & $0.6 \pm 0.3$ & $0.2 \pm 0.6$ & $1.1 \pm 0.6$ & $2.8 \pm 0.5$ & $1.1 \pm 0.6$ & 5275.7 \\
\hline $171124 \mathrm{~A}$ & $40 \pm 10$ & $8 \pm 4$ & $0.89 \pm 0.09$ & $\ldots$ & $\ldots$ & $\ldots$ & $0.89 \pm 0.09$ & 317.9 \\
\hline $171210 \mathrm{~A}$ & $0.3 \pm 0.1$ & $200 \pm 500$ & $0.7 \pm 0.3$ & $\ldots$ & $\ldots$ & $\ldots$ & $0.7 \pm 0.3$ & 1368.6 \\
\hline $180210 \mathrm{~A}$ & $1.8 \pm 0.6$ & $140 \pm 80$ & $1.0 \pm 0.2$ & $\ldots$ & $\ldots$ & $\ldots$ & $1.0 \pm 0.2$ & 1598.1 \\
\hline $180526 \mathrm{~A}$ & $3 \pm 2$ & $800 \pm 200$ & $1.3 \pm 0.7$ & $\ldots$ & $\ldots$ & $\ldots$ & $1.3 \pm 0.7$ & 1139.7 \\
\hline $180703 \mathrm{~A}$ & $6 \pm 3$ & $10 \pm 20$ & $0.8 \pm 0.2$ & $\ldots$ & $\ldots$ & $\ldots$ & $0.8 \pm 0.2$ & 1610.1 \\
\hline 180720B & $40 \pm 20$ & $62 \pm 5$ & $1.9 \pm 0.1$ & $1.5 \pm 0.2$ & $3.2 \pm 0.6$ & $2.37 \pm 0.08$ & $3.2 \pm 0.6$ & 613.3 \\
\hline
\end{tabular}


Table 6

Highest-energy Events of Fermi-LAT GRBs

\begin{tabular}{|c|c|c|c|c|c|c|c|c|}
\hline \multirow[b]{2}{*}{ GRB Name } & \multicolumn{4}{|c|}{ GBM Time Window } & \multicolumn{4}{|c|}{ Time Resolved } \\
\hline & $\begin{array}{c}\text { Events } \\
(P>0.9)\end{array}$ & $\begin{array}{l}\text { Energy } \\
(\mathrm{GeV})\end{array}$ & $\begin{array}{l}\text { Arrival Time } \\
\text { (s) }\end{array}$ & Probability & $\begin{array}{l}\text { Events } \\
(P>0.9)\end{array}$ & $\begin{array}{l}\text { Energy } \\
(\mathrm{GeV})\end{array}$ & $\begin{array}{l}\text { Arrival Time } \\
\text { (s) }\end{array}$ & Probability \\
\hline 080818B & $\ldots$ & $\ldots$ & $\ldots$ & $\ldots$ & 3 & 1.70 & 9018.86 & 1.00 \\
\hline $080825 \mathrm{C}$ & 9 & 0.30 & 21.38 & 0.93 & 13 & 0.68 & 28.28 & 1.00 \\
\hline 080916C & 237 & 27.00 & 40.50 & 1.00 & 320 & 27.00 & 40.50 & 1.00 \\
\hline 081006 & 8 & 0.71 & 1.80 & 1.00 & 12 & 0.80 & 12.08 & 0.99 \\
\hline 081009 & $\ldots$ & $\ldots$ & $\ldots$ & $\ldots$ & 9 & 1.60 & 1250.13 & 0.99 \\
\hline 081024B & 6 & 0.45 & 0.26 & 1.00 & 12 & 1.70 & 2.12 & 1.00 \\
\hline 081102B & 6 & 0.74 & 1.33 & 0.97 & 5 & 0.55 & 0.30 & 1.00 \\
\hline $081122 \mathrm{~A}$ & 1 & 0.15 & 5.05 & 0.93 & 4 & 2.50 & 66.53 & 1.00 \\
\hline 081203A & $\ldots$ & $\ldots$ & $\ldots$ & $\ldots$ & 5 & 1.00 & 379.66 & 1.00 \\
\hline 081224 & $\ldots$ & $\ldots$ & $\ldots$ & $\ldots$ & 4 & 1.80 & 177.49 & 1.00 \\
\hline 090102 & $\ldots$ & $\ldots$ & $\ldots$ & $\ldots$ & 2 & 0.58 & 3915.89 & 0.99 \\
\hline 090217 & 19 & 0.82 & 14.83 & 1.00 & 23 & 0.82 & 14.83 & 1.00 \\
\hline 090227A & 1 & 0.43 & 2.90 & 1.00 & 4 & 2.30 & 51.68 & 1.00 \\
\hline 090227B & 2 & 0.13 & 0.21 & 1.00 & $\ldots$ & $\ldots$ & $\ldots$ & $\ldots$ \\
\hline 090228A & 1 & 0.14 & 0.09 & 1.00 & 5 & 0.70 & 2.09 & 0.92 \\
\hline 090323 & 16 & 1.20 & 22.57 & 0.99 & 59 & 7.40 & 195.42 & 1.00 \\
\hline 090328 & 12 & 2.30 & 53.30 & 1.00 & 67 & 5.50 & 697.80 & 1.00 \\
\hline 090427A & $\ldots$ & $\ldots$ & $\ldots$ & $\ldots$ & 2 & 14.00 & 422.87 & 1.00 \\
\hline 090510 & 64 & 30.00 & 0.83 & 1.00 & 260 & 30.00 & 0.83 & 1.00 \\
\hline 090626 & 3 & 0.12 & 9.00 & 0.99 & 20 & 2.10 & 111.63 & 1.00 \\
\hline 090720B & 6 & 0.43 & 1.68 & 0.99 & 5 & 0.43 & 1.68 & 1.00 \\
\hline 090902B & 245 & 14.00 & 14.16 & 1.00 & 480 & 40.00 & 81.74 & 1.00 \\
\hline 090926A & 252 & 3.30 & 9.48 & 1.00 & 410 & 19.00 & 24.83 & 1.00 \\
\hline 091003 & 5 & 2.70 & 6.47 & 1.00 & 22 & 5.90 & 348.63 & 1.00 \\
\hline 091031 & 9 & 0.60 & 6.44 & 1.00 & 25 & 1.40 & 408.18 & 1.00 \\
\hline 091120 & 3 & 0.20 & 31.79 & 0.99 & 10 & 7.00 & 712.58 & 1.00 \\
\hline 091127 & 1 & 1.60 & 8.61 & 1.00 & 3 & 2.20 & 16.94 & 1.00 \\
\hline 100116A & 8 & 0.84 & 101.30 & 0.99 & 20 & 33.00 & 378.98 & 1.00 \\
\hline $100213 \mathrm{C}$ & $\ldots$ & $\ldots$ & $\ldots$ & $\ldots$ & 3 & 34.00 & 3389.03 & 1.00 \\
\hline $100225 \mathrm{~A}$ & 6 & 0.34 & 6.62 & 0.97 & 8 & 3.00 & 64.85 & 1.00 \\
\hline $100325 \mathrm{~A}$ & 6 & 0.82 & 0.34 & 1.00 & 6 & 0.82 & 0.34 & 1.00 \\
\hline 100414A & 4 & 0.47 & 24.50 & 1.00 & 48 & 30.00 & 33.36 & 1.00 \\
\hline 100423B & $\ldots$ & $\ldots$ & $\ldots$ & $\ldots$ & 3 & 3.90 & 166.69 & 1.00 \\
\hline $100511 \mathrm{~A}$ & 3 & 0.60 & 11.61 & 0.92 & 19 & 46.00 & 161.90 & 1.00 \\
\hline $100620 \mathrm{~A}$ & 5 & 0.25 & 3.75 & 1.00 & 5 & 0.25 & 3.75 & 1.00 \\
\hline 100724B & 8 & 0.16 & 42.83 & 0.96 & 8 & 0.16 & 42.83 & 0.98 \\
\hline $100728 \mathrm{~A}$ & $\ldots$ & $\ldots$ & $\ldots$ & $\ldots$ & 7 & 3.10 & 325.06 & 1.00 \\
\hline $100826 \mathrm{~A}$ & 5 & 1.70 & 61.39 & 1.00 & 4 & 1.70 & 61.39 & 1.00 \\
\hline $101014 \mathrm{~A}$ & $\ldots$ & $\ldots$ & $\ldots$ & $\ldots$ & 6 & 14.00 & 2750.71 & 1.00 \\
\hline $101107 \mathrm{~A}$ & 6 & 4.80 & 139.85 & 1.00 & 7 & 4.80 & 139.85 & 1.00 \\
\hline 101227B & 3 & 1.50 & 20.53 & 1.00 & 3 & 1.50 & 20.53 & 1.00 \\
\hline $110120 \mathrm{~A}$ & 2 & 0.47 & 0.87 & 0.99 & 10 & 2.00 & 72.46 & 1.00 \\
\hline $110123 \mathrm{~A}$ & $\ldots$ & $\ldots$ & $\ldots$ & $\ldots$ & 7 & 2.10 & 445.55 & 1.00 \\
\hline $110213 \mathrm{~A}$ & $\ldots$ & $\ldots$ & $\ldots$ & $\ldots$ & 2 & 3.00 & 1261.39 & 1.00 \\
\hline 110328B & 1 & 0.16 & 9.84 & 0.90 & 6 & 4.00 & 328.67 & 1.00 \\
\hline $110428 \mathrm{~A}$ & 1 & 0.62 & 7.28 & 0.94 & 9 & 3.00 & 14.80 & 0.94 \\
\hline $110518 \mathrm{~A}$ & $\ldots$ & $\ldots$ & $\ldots$ & $\ldots$ & 4 & 2.90 & 2039.24 & 1.00 \\
\hline $110529 \mathrm{~A}$ & 1 & 0.10 & 0.07 & 1.00 & $\ldots$ & $\ldots$ & $\ldots$ & $\ldots$ \\
\hline $110625 \mathrm{~A}$ & $\ldots$ & $\ldots$ & $\ldots$ & $\ldots$ & 7 & 1.70 & 577.24 & 0.95 \\
\hline $110721 \mathrm{~A}$ & 33 & 6.70 & 4.50 & 1.00 & 36 & 6.70 & 4.50 & 1.00 \\
\hline $110728 \mathrm{~A}$ & 1 & 0.51 & 0.36 & 1.00 & 6 & 1.40 & 2.98 & 1.00 \\
\hline $110731 \mathrm{~A}$ & 48 & 0.97 & 5.52 & 1.00 & 75 & 3.50 & 435.96 & 1.00 \\
\hline $110903 \mathrm{~A}$ & 3 & 16.00 & 301.35 & 1.00 & 4 & 16.00 & 301.35 & 1.00 \\
\hline 110921B & 4 & 0.65 & 13.08 & 0.99 & 10 & 2.00 & 202.48 & 1.00 \\
\hline 111210B & 3 & 0.15 & 47.63 & 0.99 & 7 & 0.56 & 96.10 & 1.00 \\
\hline $120107 \mathrm{~A}$ & 7 & 1.90 & 7.70 & 1.00 & 8 & 1.90 & 7.70 & 1.00 \\
\hline $120226 \mathrm{~A}$ & 3 & 0.27 & 30.71 & 0.96 & 10 & 0.38 & 283.65 & 0.96 \\
\hline $120316 \mathrm{~A}$ & 3 & 2.10 & 26.44 & 1.00 & 10 & 2.10 & 26.44 & 1.00 \\
\hline 120420B & $\ldots$ & $\ldots$ & $\ldots$ & $\ldots$ & 4 & 1.90 & 3800.54 & 1.00 \\
\hline $120526 \mathrm{~A}$ & $\ldots$ & $\ldots$ & $\ldots$ & $\ldots$ & 20 & 14.00 & 1354.30 & 1.00 \\
\hline $120624 \mathrm{~B}$ & 4 & 0.36 & -201.87 & 0.91 & 110 & 1.90 & 557.66 & 1.00 \\
\hline $120709 \mathrm{~A}$ & 12 & 2.20 & 1.77 & 1.00 & 22 & 2.60 & 140.33 & 0.99 \\
\hline
\end{tabular}


Table 6

(Continued)

\begin{tabular}{|c|c|c|c|c|c|c|c|c|}
\hline \multirow[b]{2}{*}{ GRB Name } & \multicolumn{4}{|c|}{ GBM Time Window } & \multicolumn{4}{|c|}{ Time Resolved } \\
\hline & $\begin{array}{l}\text { Events } \\
(P>0.9)\end{array}$ & $\begin{array}{l}\text { Energy } \\
(\mathrm{GeV})\end{array}$ & $\begin{array}{l}\text { Arrival Time } \\
\text { (s) }\end{array}$ & Probability & $\begin{array}{c}\text { Events } \\
(P>0.9)\end{array}$ & $\begin{array}{l}\text { Energy } \\
(\mathrm{GeV})\end{array}$ & $\begin{array}{l}\text { Arrival Time } \\
\text { (s) }\end{array}$ & Probability \\
\hline $120711 \mathrm{~A}$ & $\ldots$ & $\ldots$ & $\ldots$ & $\ldots$ & 29 & 2.50 & 5431.65 & 1.00 \\
\hline 120729A & $\ldots$ & $\ldots$ & $\ldots$ & $\ldots$ & 3 & 2.40 & 396.87 & 1.00 \\
\hline $120830 \mathrm{~A}$ & 3 & 0.48 & 0.75 & 1.00 & 6 & 0.78 & 2.96 & 1.00 \\
\hline 120911B & 50 & 1.20 & 15.51 & 0.96 & 66 & 1.40 & 90.29 & 1.00 \\
\hline $120915 \mathrm{~A}$ & 2 & 0.18 & 0.14 & 1.00 & 2 & 0.25 & 0.82 & 0.99 \\
\hline 120919B & $\ldots$ & $\ldots$ & $\cdots$ & $\ldots$ & 3 & 13.00 & 605.27 & 1.00 \\
\hline $121029 \mathrm{~A}$ & 1 & 0.12 & 13.80 & 0.94 & 5 & 4.30 & 1926.46 & 1.00 \\
\hline 121123B & $\ldots$ & $\ldots$ & $\ldots$ & $\ldots$ & 7 & 2.50 & 268.02 & 1.00 \\
\hline $121225 B$ & 1 & 0.12 & 11.04 & 0.94 & $\ldots$ & $\ldots$ & $\ldots$ & $\ldots$ \\
\hline $130310 \mathrm{~A}$ & 2 & 0.84 & 4.12 & 1.00 & 11 & 1.40 & 329.90 & 1.00 \\
\hline $130325 \mathrm{~A}$ & 1 & 0.23 & 2.19 & 0.99 & 4 & 5.30 & 828.57 & 1.00 \\
\hline 130327B & 7 & 4.60 & 20.23 & 1.00 & 42 & 9.20 & 49.42 & 1.00 \\
\hline 130427A & 252 & 77.00 & 18.64 & 1.00 & 600 & 94.00 & 243.13 & 1.00 \\
\hline 130502B & 21 & 2.00 & 27.47 & 1.00 & 68 & 31.00 & 222.10 & 0.97 \\
\hline $130504 \mathrm{C}$ & 7 & 2.20 & 54.16 & 1.00 & 19 & 5.70 & 250.82 & 1.00 \\
\hline 130518A & 8 & 0.39 & 50.71 & 0.94 & 32 & 2.10 & 270.81 & 1.00 \\
\hline 130606B & $\ldots$ & $\ldots$ & $\ldots$ & $\ldots$ & 11 & 4.60 & 527.27 & 1.00 \\
\hline $130702 \mathrm{~A}$ & $\ldots$ & $\ldots$ & $\ldots$ & $\ldots$ & 3 & 1.70 & 272.29 & 0.99 \\
\hline $130821 \mathrm{~A}$ & 7 & 2.80 & 64.29 & 1.00 & 47 & 6.30 & 219.13 & 0.96 \\
\hline $130828 \mathrm{~A}$ & 32 & 1.20 & 52.09 & 1.00 & 32 & 1.20 & 52.09 & 1.00 \\
\hline 130907A & $\ldots$ & $\ldots$ & $\ldots$ & $\ldots$ & 2 & 5.60 & 3618.37 & 1.00 \\
\hline 131014A & 10 & 1.20 & 1.98 & 1.00 & 23 & 1.80 & 14.37 & 0.98 \\
\hline $131029 \mathrm{~A}$ & 23 & 1.20 & 71.66 & 1.00 & 35 & 3.20 & 189.93 & 1.00 \\
\hline $131108 \mathrm{~A}$ & 135 & 1.20 & 5.21 & 1.00 & 200 & 1.50 & 66.33 & 1.00 \\
\hline 131209A & 2 & 0.22 & 15.56 & 0.92 & 8 & 0.29 & 65.13 & 0.91 \\
\hline $131231 \mathrm{~A}$ & 5 & 0.96 & 37.94 & 1.00 & 35 & 48.00 & 110.29 & 1.00 \\
\hline $140102 \mathrm{~A}$ & 3 & 0.37 & 3.42 & 0.99 & 12 & 2.30 & 4.29 & 1.00 \\
\hline 140104B & $\ldots$ & $\ldots$ & $\ldots$ & $\ldots$ & 11 & 3.10 & 809.91 & 1.00 \\
\hline $140110 \mathrm{~A}$ & 29 & 2.00 & 0.62 & 1.00 & 38 & 2.00 & 0.62 & 1.00 \\
\hline $140124 \mathrm{~A}$ & 1 & 2.30 & 93.56 & 0.96 & 3 & 2.30 & 93.56 & 0.93 \\
\hline 140206B & 24 & 0.75 & 24.00 & 1.00 & 62 & 11.00 & 6735.90 & 1.00 \\
\hline 140219A & $\ldots$ & $\ldots$ & $\ldots$ & $\ldots$ & 4 & 1.70 & 1356.56 & 1.00 \\
\hline $140323 \mathrm{~A}$ & $\ldots$ & $\ldots$ & $\ldots$ & $\ldots$ & 4 & 2.80 & 226.80 & 1.00 \\
\hline $140402 \mathrm{~A}$ & 4 & 0.66 & 0.05 & 1.00 & 16 & 3.70 & 8.73 & 1.00 \\
\hline $140416 \mathrm{~A}$ & $\ldots$ & $\ldots$ & $\ldots$ & $\ldots$ & 3 & 10.00 & 2207.37 & 1.00 \\
\hline $140523 \mathrm{~A}$ & 14 & 2.60 & 18.87 & 1.00 & 35 & 7.30 & 43.46 & 1.00 \\
\hline $140528 \mathrm{~A}$ & $\ldots$ & $\ldots$ & $\ldots$ & $\ldots$ & 3 & 4.60 & 1377.90 & 1.00 \\
\hline 140619B & 21 & 23.00 & 0.61 & 1.00 & 23 & 23.00 & 0.61 & 1.00 \\
\hline $140723 \mathrm{~A}$ & 18 & 1.00 & 0.56 & 0.99 & 31 & 1.00 & 0.56 & 1.00 \\
\hline $140729 \mathrm{~A}$ & 9 & 1.30 & 44.14 & 1.00 & 11 & 1.50 & 73.40 & 1.00 \\
\hline $140810 \mathrm{~A}$ & $\ldots$ & $\ldots$ & $\ldots$ & $\ldots$ & 13 & 15.00 & 1490.23 & 1.00 \\
\hline $140825 \mathrm{~A}$ & $\ldots$ & $\ldots$ & $\ldots$ & $\ldots$ & 3 & 1.60 & 1702.32 & 0.91 \\
\hline 140928A & $\ldots$ & $\ldots$ & $\ldots$ & $\ldots$ & 4 & 52.00 & 2554.67 & 1.00 \\
\hline 141012A & 7 & 1.00 & 9.64 & 1.00 & 14 & 1.10 & 19.51 & 1.00 \\
\hline $141028 \mathrm{~A}$ & 19 & 0.68 & 32.21 & 1.00 & 34 & 3.90 & 157.52 & 0.94 \\
\hline $141102 \mathrm{~A}$ & 1 & 0.15 & 2.54 & 0.92 & 7 & 0.64 & 30.91 & 0.97 \\
\hline $141113 \mathrm{~A}$ & 4 & 0.53 & 0.07 & 1.00 & 5 & 0.53 & 0.07 & 1.00 \\
\hline $141207 \mathrm{~A}$ & 39 & 3.40 & 4.80 & 1.00 & 48 & 5.50 & 734.33 & 1.00 \\
\hline $141221 \mathrm{~B}$ & 1 & 0.11 & 22.17 & 0.98 & 3 & 5.50 & 58.47 & 1.00 \\
\hline $141222 \mathrm{~A}$ & $\ldots$ & $\ldots$ & $\ldots$ & $\ldots$ & 11 & 3.60 & 227.42 & 1.00 \\
\hline 150118B & $\ldots$ & $\ldots$ & $\ldots$ & $\ldots$ & 3 & 1.80 & 51.03 & 1.00 \\
\hline $150202 B$ & 2 & 1.00 & 115.70 & 1.00 & 2 & 1.00 & 115.70 & 0.99 \\
\hline $150210 \mathrm{~A}$ & 15 & 1.00 & 2.02 & 1.00 & 18 & 2.50 & 169.37 & 1.00 \\
\hline 150314A & $\ldots$ & $\ldots$ & $\ldots$ & $\ldots$ & 13 & 1.90 & 3064.28 & 0.97 \\
\hline $150403 \mathrm{~A}$ & $\ldots$ & $\ldots$ & $\ldots$ & $\ldots$ & 5 & 5.40 & 631.76 & 1.00 \\
\hline $150510 \mathrm{~A}$ & 5 & 0.58 & 2.43 & 1.00 & 9 & 2.00 & 100.91 & 1.00 \\
\hline $150513 \mathrm{~A}$ & 8 & 2.20 & -56.40 & 1.00 & $\ldots$ & $\ldots$ & $\ldots$ & $\ldots$ \\
\hline $150514 \mathrm{~A}$ & 1 & 0.18 & 3.58 & 0.99 & 2 & 6.20 & 442.44 & 1.00 \\
\hline $150523 \mathrm{~A}$ & 23 & 1.90 & 42.33 & 1.00 & 44 & 7.00 & 118.00 & 1.00 \\
\hline $150627 \mathrm{~A}$ & $\ldots$ & $\ldots$ & $\ldots$ & $\ldots$ & 26 & 8.10 & 258.66 & 1.00 \\
\hline $150702 \mathrm{~A}$ & $\ldots$ & $\ldots$ & $\ldots$ & $\ldots$ & 6 & 0.80 & 1115.33 & 0.99 \\
\hline $150902 \mathrm{~A}$ & 18 & 0.41 & 6.18 & 0.99 & 40 & 11.00 & 97.49 & 1.00 \\
\hline
\end{tabular}


Table 6

(Continued)

\begin{tabular}{|c|c|c|c|c|c|c|c|c|}
\hline \multirow[b]{2}{*}{ GRB Name } & \multicolumn{4}{|c|}{ GBM Time Window } & \multicolumn{4}{|c|}{ Time Resolved } \\
\hline & $\begin{array}{l}\text { Events } \\
(P>0.9)\end{array}$ & $\begin{array}{l}\text { Energy } \\
(\mathrm{GeV})\end{array}$ & $\begin{array}{l}\text { Arrival Time } \\
\text { (s) }\end{array}$ & Probability & $\begin{array}{l}\text { Events } \\
(P>0.9)\end{array}$ & $\begin{array}{l}\text { Energy } \\
(\mathrm{GeV})\end{array}$ & $\begin{array}{l}\text { Arrival Time } \\
\text { (s) }\end{array}$ & Probability \\
\hline 160310A & $\ldots$ & $\ldots$ & $\ldots$ & $\ldots$ & 2 & 1.40 & 99.25 & 0.95 \\
\hline 160314B & $\ldots$ & $\ldots$ & $\ldots$ & $\cdots$ & 4 & 0.88 & 628.72 & 0.94 \\
\hline $160325 \mathrm{~A}$ & 2 & 0.31 & 4.98 & 0.96 & 21 & 3.00 & 92.02 & 1.00 \\
\hline $160422 \mathrm{~A}$ & $\ldots$ & $\ldots$ & $\ldots$ & $\ldots$ & 2 & 12.00 & 769.62 & 1.00 \\
\hline $160503 \mathrm{~A}$ & $\ldots$ & $\ldots$ & $\ldots$ & $\cdots$ & 11 & 0.52 & 12969.55 & 0.91 \\
\hline $160509 \mathrm{~A}$ & 103 & 52.00 & 76.51 & 1.00 & 140 & 52.00 & 76.51 & 1.00 \\
\hline $160521 \mathrm{~B}$ & $\ldots$ & $\ldots$ & $\ldots$ & $\ldots$ & 8 & 13.00 & 422.62 & 1.00 \\
\hline $160623 \mathrm{~A}$ & $\cdots$ & $\cdots$ & $\cdots$ & $\ldots$ & 43 & 18.00 & 12038.53 & 1.00 \\
\hline $160625 \mathrm{~B}$ & 250 & 15.00 & 346.18 & 1.00 & 260 & 15.00 & 346.18 & 1.00 \\
\hline $160702 \mathrm{~A}$ & $\ldots$ & $\ldots$ & $\ldots$ & $\ldots$ & 3 & 4.80 & 1941.52 & 1.00 \\
\hline $160709 \mathrm{~A}$ & 24 & 0.99 & 1.47 & 1.00 & 28 & 0.99 & 1.47 & 1.00 \\
\hline $160816 \mathrm{~A}$ & 15 & 1.10 & 1.40 & 1.00 & 46 & 9.20 & 1094.77 & 1.00 \\
\hline $160821 \mathrm{~A}$ & 35 & 0.68 & 156.93 & 0.94 & 52 & 4.70 & 212.43 & 1.00 \\
\hline $160829 \mathrm{~A}$ & $\cdots$ & $\ldots$ & $\ldots$ & $\ldots$ & 3 & 9.40 & 0.95 & 1.00 \\
\hline $160905 \mathrm{~A}$ & 5 & 2.20 & 22.03 & 1.00 & 22 & 7.90 & 347.76 & 1.00 \\
\hline $160910 \mathrm{~A}$ & 3 & 0.15 & 23.57 & 0.91 & 4 & 0.49 & 197.48 & 0.99 \\
\hline $161015 \mathrm{~A}$ & 9 & 1.00 & 7.47 & 1.00 & 10 & 1.00 & 7.47 & 1.00 \\
\hline 161109A & $\cdots$ & $\ldots$ & $\cdots$ & $\ldots$ & 8 & 3.40 & 594.81 & 1.00 \\
\hline 170115B & 13 & 0.64 & 1.34 & 0.93 & 28 & 2.30 & 861.41 & 0.99 \\
\hline $170127 \mathrm{C}$ & $\ldots$ & $\ldots$ & $\ldots$ & $\ldots$ & 10 & 0.51 & 2889.00 & 1.00 \\
\hline $170214 \mathrm{~A}$ & 103 & 7.80 & 103.62 & 1.00 & 220 & 7.80 & 103.62 & 1.00 \\
\hline $170228 \mathrm{~A}$ & 5 & 0.34 & 9.47 & 0.97 & 6 & 0.34 & 9.47 & 0.97 \\
\hline 170306B & 1 & 0.23 & 21.16 & 0.99 & 6 & 0.50 & 43.25 & 1.00 \\
\hline $170329 \mathrm{~A}$ & 9 & 0.78 & 4.11 & 1.00 & 10 & 0.78 & 4.11 & 1.00 \\
\hline $170405 \mathrm{~A}$ & 7 & 0.16 & 41.82 & 0.93 & 23 & 0.89 & 445.97 & 0.98 \\
\hline 170409A & $\ldots$ & $\ldots$ & $\ldots$ & $\ldots$ & 10 & 9.90 & 440.27 & 1.00 \\
\hline $170424 \mathrm{~A}$ & 3 & 1.10 & 54.20 & 1.00 & 7 & 1.10 & 54.20 & 1.00 \\
\hline $170510 \mathrm{~A}$ & 6 & 1.00 & 45.47 & 1.00 & 6 & 1.00 & 45.47 & 1.00 \\
\hline $170522 \mathrm{~A}$ & 6 & 3.70 & 6.86 & 1.00 & 10 & 3.70 & 6.86 & 1.00 \\
\hline $170728 \mathrm{~B}$ & 4 & 0.63 & 9.27 & 0.99 & 7 & 0.63 & 9.27 & 0.92 \\
\hline 170808B & 2 & 0.14 & 13.73 & 1.00 & 18 & 1.80 & 484.36 & 1.00 \\
\hline $170813 \mathrm{~A}$ & 3 & 0.58 & 9.52 & 1.00 & 4 & 0.96 & 265.30 & 0.96 \\
\hline $170825 B$ & 2 & 0.30 & 1.49 & 1.00 & 2 & 0.30 & 1.49 & 0.99 \\
\hline $170906 \mathrm{~A}$ & $\ldots$ & $\ldots$ & $\ldots$ & $\ldots$ & 29 & 3.60 & 203.25 & 1.00 \\
\hline 170921B & $\ldots$ & $\ldots$ & $\ldots$ & $\ldots$ & 2 & 2.70 & 901.67 & 1.00 \\
\hline $171010 \mathrm{~A}$ & $\ldots$ & $\ldots$ & $\ldots$ & $\ldots$ & 47 & 19.00 & 2890.98 & 0.99 \\
\hline $171011 \mathrm{C}$ & 3 & 0.51 & -0.09 & 1.00 & 2 & 0.16 & 42.72 & 0.99 \\
\hline $171102 \mathrm{~A}$ & 2 & 0.14 & 34.91 & 0.98 & 8 & 0.37 & 349.89 & 0.99 \\
\hline $171120 \mathrm{~A}$ & 6 & 1.60 & 5.63 & 1.00 & 29 & 3.40 & 4840.92 & 1.00 \\
\hline $171124 \mathrm{~A}$ & 20 & 3.60 & 4.08 & 1.00 & 21 & 3.60 & 4.08 & 1.00 \\
\hline $171210 \mathrm{~A}$ & 2 & 0.65 & 74.83 & 1.00 & 12 & 12.00 & 1374.49 & 1.00 \\
\hline $171212 \mathrm{~B}$ & $\ldots$ & $\ldots$ & $\ldots$ & $\ldots$ & 3 & 0.61 & 496.97 & 0.97 \\
\hline $180210 \mathrm{~A}$ & 3 & 0.47 & 31.78 & 1.00 & 32 & 7.40 & 1621.12 & 1.00 \\
\hline $180305 \mathrm{~A}$ & 1 & 0.20 & 6.18 & 0.99 & 2 & 8.90 & 1613.84 & 1.00 \\
\hline $180526 \mathrm{~A}$ & $\ldots$ & $\ldots$ & $\ldots$ & $\ldots$ & 8 & 2.20 & 1308.09 & 1.00 \\
\hline $180703 \mathrm{~A}$ & 2 & 0.21 & 5.06 & 0.99 & 13 & 0.93 & 40.46 & 1.00 \\
\hline 180703B & $\ldots$ & $\ldots$ & $\ldots$ & $\ldots$ & 5 & 1.10 & 34.22 & 1.00 \\
\hline $180718 \mathrm{~B}$ & 11 & 0.49 & 2.63 & 0.96 & 9 & 0.49 & 2.63 & 0.99 \\
\hline 180720B & 19 & 0.63 & 38.77 & 1.00 & 130 & 4.90 & 142.43 & 1.00 \\
\hline
\end{tabular}


Table 7

Fermi-LAT GRBs with Photon Energies $E>10 \mathrm{GeV}$

\begin{tabular}{|c|c|c|c|c|}
\hline GRB Name (Class) & $\begin{array}{c}\text { Energy } \\
(\mathrm{GeV})\end{array}$ & $\begin{array}{l}\text { Arrival Time } \\
\text { (s) }\end{array}$ & $z$ & $\begin{array}{c}E_{\mathrm{sf}} \\
(\mathrm{GeV})\end{array}$ \\
\hline \multirow[t]{2}{*}{ 080916C (L) } & 27.4 & 40.5 & 4.35 & 146.6 \\
\hline & 12.4 & 16.5 & & 66.3 \\
\hline 090427A (L) & 14.1 & 422.9 & $\cdots$ & $\ldots$ \\
\hline $090510(\mathrm{~S})$ & 30.0 & 0.83 & 0.90 & 56.8 \\
\hline \multirow[t]{7}{*}{ 090902B (L) } & 40.0 & 81.7 & 1.82 & 112.5 \\
\hline & 21.7 & 331.9 & & 61.2 \\
\hline & 18.1 & 26.2 & & 51.0 \\
\hline & 15.4 & 45.6 & & 43.4 \\
\hline & 14.2 & 14.2 & & 40.0 \\
\hline & 12.7 & 42.4 & & 35.8 \\
\hline & 11.9 & 11.7 & & 33.6 \\
\hline \multirow[t]{2}{*}{ 090926A (L) } & 19.4 & 24.8 & 2.11 & 60.3 \\
\hline & 10.4 & 3785.0 & & 32.3 \\
\hline \multirow[t]{2}{*}{ 100116A (L) } & 32.6 & 379.0 & $\cdots$ & $\cdots$ \\
\hline & 13.3 & 296.0 & $\ldots$ & $\ldots$ \\
\hline $100213 \mathrm{C}(\mathrm{L})$ & 34.0 & 3389.0 & $\ldots$ & $\ldots$ \\
\hline \multirow[t]{2}{*}{ 100414A (L) } & 30.0 & 33.4 & 1.37 & 70.6 \\
\hline & 25.1 & 358.5 & & 59.5 \\
\hline \multirow[t]{2}{*}{ 100511A (L) } & 46.0 & 161.9 & $\ldots$ & $\ldots$ \\
\hline & 18.4 & 179.8 & $\cdots$ & $\cdots$ \\
\hline \multirow[t]{2}{*}{ 101014A (L) } & 13.6 & 2750.7 & $\cdots$ & $\ldots$ \\
\hline & 11.2 & 2962.0 & $\ldots$ & $\ldots$ \\
\hline 110903A (L) & 15.6 & 301.0 & $\ldots$ & .. \\
\hline $120526 \mathrm{~A}(\mathrm{~L})$ & 14.3 & 1354.3 & $\cdots$ & $\cdots$ \\
\hline 120919B (L) & 12.7 & 605.3 & $\ldots$ & $\ldots$ \\
\hline \multirow[t]{17}{*}{ 130427A (L) } & 94.1 & 243.1 & 0.34 & 126.1 \\
\hline & 77.1 & 18.6 & & 103.3 \\
\hline & 57.4 & 256.0 & & 76.9 \\
\hline & 38.7 & 78.4 & & 51.9 \\
\hline & 38.2 & 3409.0 & & 51.2 \\
\hline & 33.6 & 34366.0 & & 45.0 \\
\hline & 28.4 & 47.6 & & 38.0 \\
\hline & 26.9 & 84.7 & & 36.0 \\
\hline & 25.4 & 141.0 & & 34.0 \\
\hline & 19.3 & 6062.0 & & 25.9 \\
\hline & 17.1 & 217.0 & & 22.9 \\
\hline & 14.9 & 119.3 & & 20.0 \\
\hline & 12.9 & 80.5 & & 17.3 \\
\hline & 12.2 & 64.5 & & 16.3 \\
\hline & 12.0 & 23.5 & & 16.1 \\
\hline & 11.7 & 214.0 & & 15.7 \\
\hline & 10.8 & 23.2 & & 14.5 \\
\hline \multirow[t]{2}{*}{ 130502B (L) } & 31.1 & 222.1 & $\cdots$ & $\ldots$ \\
\hline & 17.3 & 48.0 & $\cdots$ & $\ldots$ \\
\hline \multirow[t]{2}{*}{$131231 \mathrm{~A}(\mathrm{~L})$} & 48.3 & 110.3 & 0.64 & 79.2 \\
\hline & 17.1 & 844.2 & & 28.0 \\
\hline 140206B (L) & 11.0 & 6735.9 & $\cdots$ & $\cdots$ \\
\hline 140416A (L) & 10.1 & 2207.4 & $\cdots$ & $\cdots$ \\
\hline 140619B (L) & 22.7 & 0.6 & $\ldots$ & $\ldots$ \\
\hline 140810A (L) & 15.4 & 1490.0 & $\ldots$ & $\ldots$ \\
\hline 140928A (L) & 51.7 & 2554.7 & $\cdots$ & $\cdots$ \\
\hline $150902 \mathrm{~A}(\mathrm{~L})$ & 11.3 & 97.5 & $\cdots$ & $\cdots$ \\
\hline $160422 \mathrm{~A}(\mathrm{~L})$ & 12.3 & 769.6 & $\ldots$ & $\ldots$ \\
\hline \multirow[t]{2}{*}{ 160509A (L) } & 51.9 & 76.5 & 1.17 & 112.6 \\
\hline & 41.5 & 242.0 & & 90.1 \\
\hline $160521 B(\mathrm{~L})$ & 12.7 & 422.6 & $\cdots$ & $\cdots$ \\
\hline 160623A (L) & 18.2 & 12038.5 & 0.37 & 24.9 \\
\hline $160625 B(\mathrm{~L})$ & 15.3 & 346.2 & 1.41 & 36.9 \\
\hline 171010A (L) & 19.0 & 2891.0 & 0.33 & 25.3 \\
\hline 171210A (L) & 12.4 & 1374.5 & $\ldots$ & $\cdots$ \\
\hline
\end{tabular}




\section{Appendix B \\ Description of the Content of the Fits File}

All the information used to produce plots and tables in this paper is saved into a FITS file described in Table 8.

Table 8

Definition of the Columns in the Fermi GRB LAT FITS File

\begin{tabular}{|c|c|c|}
\hline Name & Units & Description \\
\hline GCNNAME & & Name as appears in the GCN distribution list \\
\hline GRBNAME & & Name of the GRB in YYMMDDFFF \\
\hline GRBDATE & & Date of the trigger \\
\hline GRBMET & $\mathrm{s}$ & Mission Elapsed Time since 2001 Jan 1 UT 00:00:00 \\
\hline $\mathrm{RA}$ & $\operatorname{deg}$ & R.A. $(J 2000)$ \\
\hline $\mathrm{DEC}$ & $\operatorname{deg}$ & decl. (J2000) \\
\hline ERR & $\operatorname{deg}$ & Localization error from LTF analysis \\
\hline REDSHIFT & & Redshift of the GRB \\
\hline LUMINOSITY_DISTANCE & $\mathrm{cm}$ & Luminosity distance calculated using the redshift of the GRB (when available) \\
\hline THETA & $\operatorname{deg}$ & Off-axis angle at the time of the trigger \\
\hline ZENITH & deg & Zenith angle at the time of the trigger \\
\hline ARR & & If the GRB triggered an ARR, this flag is set to 1 ; otherwise, it is set to 0 \\
\hline DISTANCE2CLOSEST & $\operatorname{deg}$ & Distance to the closest $3 \mathrm{FGL}$ source \\
\hline IRES & & Instrument response function used in the analysis \\
\hline GBMT0 5 & $\mathrm{~s}$ & GBM $T_{05}$, when available; otherwise, this number is set to 0 \\
\hline GBMT 90 & $\mathrm{~s}$ & $\begin{array}{l}\text { GBM } T_{90} \text {, when available; otherwise, this number corresponds to the estimated duration of the } \\
\text { prompt emission }\end{array}$ \\
\hline GBMT 95 & $\mathrm{~s}$ & Calclated as GBMT05+GBMT 90 \\
\hline LLEBBBD_SIG & sigma & Significance of the signal in the LLE data \\
\hline LLEBBBD_SIG_DETECTED & & Whether the GRB is detected in LLE data \\
\hline LLET05 & $\mathrm{s}$ & LLE onset time $\left(T_{\mathrm{LLE}, 05}\right)$ \\
\hline LLET 90 & s & LLE duration $\left(T_{\text {LLE,90 }}\right)$ \\
\hline LLET95 & s & LLE end time $\left(T_{\text {LLE,95 }}\right)$ \\
\hline TLO & s & LAT emission estimated onset time $T_{\mathrm{LAT}, 0}$ \\
\hline TL100 & s & LAT emission estimated duration $T_{\mathrm{LAT}, 100}$ \\
\hline TL1 & s & LAT emission estimated end $T_{\mathrm{LAT}, 1}$ \\
\hline TLO_L & s & Estimated lower limit on the LAT emission onset time $T_{\mathrm{LAT}, 0}$ \\
\hline TL100_ERR & $\mathrm{s}$ & Estimated error on the LAT emission duration $T_{\mathrm{LAT}, 100}$ \\
\hline TL1_U & s & Estimated upper limit on LAT emission end $T_{\mathrm{LAT}, 1}$ \\
\hline LIKE_BEST_TO & s & Start of the time window for the likelihood analysis that returned the highest TS value \\
\hline LIKE_BEST_T1 & $\mathrm{s}$ & End of the time window for the likelihood analysis that returned the highest TS value \\
\hline LIKE_BEST_TS_GRB & & TS value obtained by the likelihood analysis that returned the highest TS value \\
\hline LIKE_BEST_FLUX & $\mathrm{ph} / \mathrm{cm}^{2} / \mathrm{s}$ & Flux obtained by the likelihood analysis that returned the highest TS value \\
\hline LIKE_BEST_FLUX_ERR & $\mathrm{ph} / \mathrm{cm}^{2} / \mathrm{s}$ & Estimated error on the flux obtained by the likelihood analysis that returned the highest TS value \\
\hline LIKE_BEST_FLUX_ENE & $\mathrm{erg} \mathrm{cm}^{-2} \mathrm{~s}^{-1}$ & Energy flux obtained by the likelihood analysis that returned the highest TS value \\
\hline LIKE_BEST_FLUX_ENE_ERR & $\mathrm{erg} \mathrm{cm}^{-2} \mathrm{~s}^{-1}$ & $\begin{array}{l}\text { Estimated error on the energy flux obtained by the likelihood analysis that returned the highest } \\
\text { TS value }\end{array}$ \\
\hline LIKE_BEST_FLUENCE_ENE & $\operatorname{erg~\mathrm {cm}^{-2}}$ & Energy fluence obtained by the likelihood analysis that returned the highest TS value \\
\hline LIKE_BEST_FLUENCE_ENE_ERR & $\mathrm{erg} \mathrm{cm}^{-2} \mathrm{~s}^{-1}$ & $\begin{array}{l}\text { Estimated error on the energy fluence obtained by the likelihood analysis that returned the } \\
\text { highest TS value }\end{array}$ \\
\hline LIKE_BEST_GRBindex & & $\begin{array}{l}\text { Photon index of the PL that models the GRB in the likelihood analysis that returned the highest } \\
\text { TS value }\end{array}$ \\
\hline LIKE_BEST_GRBindex_ERR & & $\begin{array}{l}\text { Estimated error on the photon index of the PL that models the GRB in the likelihood analysis } \\
\text { that returned the highest TS value }\end{array}$ \\
\hline LIKE_BEST_EISO52_RF & $10^{52} \mathrm{erg}$ & $\begin{array}{l}\text { Rest-frame isotropic energy from } 100 \mathrm{MeV} \text { to } 10 \mathrm{GeV} \text { in the likelihood analysis that returned the } \\
\text { highest TS value }\end{array}$ \\
\hline LIKE_BEST_EISO52_RF_ERR & $10^{52} \mathrm{erg}$ & $\begin{array}{l}\text { Estimated error on the rest-frame isotropic energy from } 100 \mathrm{MeV} \text { to } 10 \mathrm{GeV} \text { in the likelihood } \\
\text { analysis that returned the highest TS value }\end{array}$ \\
\hline LIKE_LAT_TO & s & Start of the time window for the likelihood analysis in the LAT time window \\
\hline LIKE_LAT_T1 & s & End of the time window for the likelihood analysis in the LAT time window \\
\hline LIKE_LAT_TS_GRB & & TS value obtained by the likelihood analysis in the LAT time window \\
\hline LIKE_LAT_FLUX & $\mathrm{ph} / \mathrm{cm}^{2} / \mathrm{s}$ & Flux obtained by the likelihood analysis in the LAT time window \\
\hline LIKE_LAT_ELUX_ERR & $\mathrm{ph} / \mathrm{cm}^{2} / \mathrm{s}$ & Estimated error on the flux obtained by the likelihood analysis in the LAT time window \\
\hline LIKE_LAT_FLUX_ENE & $\mathrm{erg} \mathrm{cm}^{-2} \mathrm{~s}^{-1}$ & Energy flux obtained by the likelihood analysis in the LAT time window \\
\hline LIKE_LAT_ELUX_ENE_ERR & $\mathrm{erg} \mathrm{cm}^{-2} \mathrm{~s}^{-1}$ & Estimated error on the energy flux obtained by the likelihood analysis in the LAT time window \\
\hline LIKE_LAT_FLUENCE_ENE & $\mathrm{erg} \mathrm{cm}^{-2}$ & Energy fluence obtained by the likelihood analysis in the LAT time window \\
\hline
\end{tabular}


Table 8

(Continued)

\begin{tabular}{|c|c|c|}
\hline Name & Units & Description \\
\hline LIKE_LAT_FLUENCE_ENE_ERR & $\mathrm{erg} \mathrm{cm}^{-2} \mathrm{~s}^{-1}$ & $\begin{array}{l}\text { Estimated error on the energy fluence obtained by the likelihood analysis in the LAT time } \\
\text { window }\end{array}$ \\
\hline LIKE_LAT_GRBindex & & Photon index of the PL that models the GRB in the likelihood analysis in the LAT time window \\
\hline LIKE_LAT_GRBindex_ERR & & $\begin{array}{l}\text { Estimated error on the photon index of the PL that models the GRB in the likelihood analysis in } \\
\text { the LAT time window }\end{array}$ \\
\hline LIKE_LAT_EISO52_RF & $10^{52} \mathrm{erg}$ & $\begin{array}{l}\text { Rest-frame isotropic energy from } 100 \mathrm{MeV} \text { to } 10 \mathrm{GeV} \text { in the likelihood analysis in the LAT time } \\
\text { window }\end{array}$ \\
\hline LIKE_LAT_EISO52_RF_ERR & $10^{52} \mathrm{erg}$ & $\begin{array}{l}\text { Estimated error on the rest-frame isotropic energy from } 100 \mathrm{MeV} \text { to } 10 \mathrm{GeV} \text { in the likelihood } \\
\text { analysis in the LAT time window }\end{array}$ \\
\hline LIKE_GBM_TO & $\mathrm{s}$ & Start of the time window for the likelihood analysis in the GBM time window \\
\hline LIKE_GBM_T1 & $\mathrm{s}$ & End of the time window for the likelihood analysis in the GBM time window \\
\hline LIKE_GBM_TS_GRB & & Value of the TSs obtained by the likelihood analysis in the GBM time window \\
\hline LIKE_GBM_FLUX & $\mathrm{ph} / \mathrm{cm}^{2} / \mathrm{s}$ & Flux obtained by the likelihood analysis in the GBM time window \\
\hline LIKE_GBM_FLUX_ERR & $\mathrm{ph} / \mathrm{cm}^{2} / \mathrm{s}$ & Estimated error on the flux obtained by the likelihood analysis in the GBM time window \\
\hline LIKE_GBM_FLUX_ENE & $\mathrm{erg} \mathrm{cm}^{-2} \mathrm{~s}^{-1}$ & Energy flux obtained by the likelihood analysis in the GBM time window \\
\hline LIKE_GBM_FLUX_ENE_ERR & $\mathrm{erg} \mathrm{cm}^{-2} \mathrm{~s}^{-1}$ & Estimated error on the energy flux obtained by the likelihood analysis in the GBM time window \\
\hline LIKE_GBM_FLUENCE_ENE & $\mathrm{erg} \mathrm{cm}^{-2}$ & Energy fluence obtained by the likelihood analysis in the GBM time window \\
\hline LIKE_GBM_FLUENCE_ENE_ERR & $\mathrm{erg} \mathrm{cm}^{-2} \mathrm{~s}^{-1}$ & $\begin{array}{l}\text { Estimated error on the energy fluence obtained by the likelihood analysis in the GBM time } \\
\text { window }\end{array}$ \\
\hline LIKE_GBM_GRBindex & & Photon index of the PL that models the GRB in the likelihood analysis in the GBM time window \\
\hline LIKE_GBM_GRBindex_ERR & & $\begin{array}{l}\text { Estimated error on the photon index of the PL that models the GRB in the likelihood analysis in } \\
\text { the GBM time window }\end{array}$ \\
\hline LIKE_GBM_EISO52_RF & $10^{52} \mathrm{erg}$ & $\begin{array}{l}\text { Rest-frame isotropic energy from } 100 \mathrm{MeV} \text { to } 10 \mathrm{GeV} \text { in the likelihood analysis in the GBM } \\
\text { time window }\end{array}$ \\
\hline LIKE_GBM_EISO52_RF_ERR & $10^{52} \mathrm{erg}$ & $\begin{array}{l}\text { Estimated error on the rest-frame isotropic energy from } 100 \mathrm{MeV} \text { to } 10 \mathrm{GeV} \text { in the likelihood } \\
\text { analysis in the GBM time window }\end{array}$ \\
\hline LIKE_EXT_TO & $\mathrm{s}$ & Start of the time window for the likelihood analysis in the EXT time window \\
\hline LIKE_EXT_T1 & s & End of the time window for the likelihood analysis in the EXT time window \\
\hline LIKE_EXT_TS_GRB & & Value of the TSs obtained by the likelihood analysis in the EXT time window \\
\hline LIKE_EXT_ELUX & $\mathrm{ph} / \mathrm{cm}^{2} / \mathrm{s}$ & Flux obtained by the likelihood analysis in the EXT time window \\
\hline LIKE_EXT_ELUX_ERR & $\mathrm{ph} / \mathrm{cm}^{2} / \mathrm{s}$ & Estimated error on the flux obtained by the likelihood analysis in the EXT time window \\
\hline LIKE_EXT_FLUX_ENE & $\mathrm{erg} \mathrm{cm}^{-2} \mathrm{~s}^{-1}$ & Energy flux obtained by the likelihood analysis in the EXT time window \\
\hline LIKE_EXT_FLUX_ENE_ERR & $\mathrm{erg} \mathrm{cm}^{-2} \mathrm{~s}^{-1}$ & Estimated error on the energy flux obtained by the likelihood analysis in the EXT time window \\
\hline LIKE_EXT_FLUENCE_ENE & $\mathrm{erg} \mathrm{cm}^{-2}$ & Energy fluence obtained by the likelihood analysis in the EXT time window \\
\hline LIKE_EXT_ELUENCE_ENE_ERR & $\mathrm{erg} \mathrm{cm}^{-2} \mathrm{~s}^{-1}$ & $\begin{array}{l}\text { Estimated error on the energy fluence obtained by the likelihood analysis in the EXT time } \\
\text { window }\end{array}$ \\
\hline LIKE_EXT_GRBindex & & Photon index of the PL that models the GRB in the likelihood analysis in the EXT time window \\
\hline LIKE_EXT_GRBindex_ERR & & $\begin{array}{l}\text { Estimated error on the photon index of the PL that models the GRB in the likelihood analysis in } \\
\text { the EXT time window }\end{array}$ \\
\hline LIKE_EXT_EISO52_RF & $10^{52} \mathrm{erg}$ & $\begin{array}{l}\text { Rest-frame isotropic energy from } 100 \mathrm{MeV} \text { to } 10 \mathrm{GeV} \text { in the likelihood analysis in the EXT time } \\
\text { window }\end{array}$ \\
\hline LIKE_EXT_EISO52_RF_ERR & $10^{52} \mathrm{erg}$ & $\begin{array}{l}\text { Estimated error on the rest-frame isotropic energy from } 100 \mathrm{MeV} \text { to } 10 \mathrm{GeV} \text { in the likelihood } \\
\text { analysis in the EXT time window }\end{array}$ \\
\hline gtsrcprob_ExtendedEmission_MAXE & $\mathrm{MeV}$ & $\begin{array}{l}\text { Maximum energy of the event with }>90 \% \text { probability to be associated with the GRB, calculated } \\
\text { during the time-resolved analysis }\end{array}$ \\
\hline gtsrcprob_ExtendedEmission_MAXE_P & & Probability of the event with maximum energy calculated during the time-resolved analysis \\
\hline gtsrcprob_ExtendedEmission_MAXE_T & $\mathrm{s}$ & Arrival time of the event with maximum energy calculated during the time-resolved analysis \\
\hline gtsrcprob_ExtendedEmission_NTH & & $\begin{array}{l}\text { Number of events with probability }>90 \% \text { to be associated with the GRB, calculated during the } \\
\text { time-resolved analysis }\end{array}$ \\
\hline LAT_BPL_CHI2 & & Value of the $\chi^{2}$ obtained by fitting the LAT light curve with a BPL model \\
\hline LAT_BPL_FO & & Normalization of the BPL model \\
\hline LAT_BPL_FO_ERR & & Error on the normalization of the BPL model \\
\hline LAT_BPL_IN1 & & First index of the BPL model \\
\hline LAT_BPL_IN1_ERR & & Error on the first index of the BPL model \\
\hline LAT_BPL_IN2 & & Second index of the BPL model \\
\hline LAT_BPL_IN2_ERR & & Error on the second index of the BPL model \\
\hline LAT_BPL_TB & & Time of the break \\
\hline LAT_BPL_TB_ERR & & Error on the time of the break \\
\hline LAT_SPL_CHI2 & & Value of the $\chi^{2}$ obtained by fitting the LAT light curve with a simple PL model \\
\hline LAT_SPL_IN1 & & Index of the simple PL model \\
\hline LAT_SPL_IN1_ERR & & Error on the index of the simple PL model \\
\hline LAT_EO & & Normalization of the simple PL model \\
\hline LAT_FO_ERR & & Error on the normalization of the simple PL model \\
\hline
\end{tabular}


Table 8

(Continued)

\begin{tabular}{|c|c|c|}
\hline Name & Units & Description \\
\hline LAT_IN_ERR & & Error on the index that best describes the behavior at late time \\
\hline GBM_assoc_key & & Name of the GRB in the Fermi GBM GRB catalog \\
\hline T90_ERROR & $\mathrm{s}$ & Error on the GBM $T_{\mathrm{GBM} .90}$ from the Fermi GBM GRB catalog \\
\hline FLUENCE & 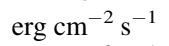 & Fluence in the $10 \mathrm{keV}-1 \mathrm{MeV}$ energy band, from the Fermi GBM GRB catalog \\
\hline LC_MEDIAN & $\mathrm{s}$ & $\begin{array}{l}\text { Variable length array containing the median points of the time interval in the time-resolved } \\
\text { analysis }\end{array}$ \\
\hline LC_END & $\mathrm{s}$ & Variable length array containing the end points of the time interval in the time-resolved analysi \\
\hline LC_ENE_ELUX & $\operatorname{erg~cm}{ }^{-2} \mathrm{~s}^{-1}$ & Variable length array containing the energy flux values in the time-resolved analysis \\
\hline LC_ENE_FLUX_ERR & 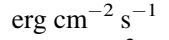 & Variable length array containing the energy flux value errors in the time-resolved analysis \\
\hline LC_INDEX_ERR & & $\begin{array}{l}\text { Variable length array containing the errors on the values of the photon index in the time-resolved } \\
\text { analysis }\end{array}$ \\
\hline LC_TS & & Variable length array containing the values of the TS in the time-resolved analysis \\
\hline
\end{tabular}

(This table is available in its entirety in FITS format.)

\section{ORCID iDs}

M. Axelsson (1) https://orcid.org/0000-0003-4378-8785

E. Bissaldi i https://orcid.org/0000-0001-9935-8106

J. W. Hewitt (1) https://orcid.org/0000-0001-5254-2248

S. Maldera (i) https://orcid.org/0000-0002-0698-4421

N. Omodei iㅏ https://orcid.org/0000-0002-5448-7577

G. Vianello (i) https://orcid.org/0000-0002-2553-0839

\section{References}

Abbott, B. P., Abbott, R., Abbott, T. D., et al. 2017a, ApJL, 848, L12 Abbott, B. P., Abbott, R., Abbott, T. D., et al. 2017b, ApJL, 848, L13 Abbott, B. P., Abbott, R., Abbott, T. D., et al. 2017c, PhRvL, 119, 161101 Abdo, A. A., Ackermann, M., Ajello, M., et al. 2009a, ApJL, 706, L138 Abdo, A. A., Ackermann, M., Ajello, M., et al. 2009b, Natur, 462, 331 Abdo, A. A., Ackermann, M., Arimoto, M., et al. 2009c, Sci, 323, 1688 Abdo, A. A., Ackermann, M., Asano, K., et al. 2009d, APh, 32, 193 Abdo, A. A., Ackermann, M., Asano, K., et al. 2009e, ApJ, 707, 580 Abdo, A. A., Ackermann, M., Ajello, M., et al. 2010a, ApJ, 712, 558 Abdo, A. A., Ackermann, M., Ajello, M., et al. 2010b, ApJ, 723, 1082 Abdollahi, S., Ackermann, M., Ajello, M., et al. 2017, ApJ, 846, 34 Acero, F., Ackermann, M., Ajello, M., et al. 2015, ApJS, 218, 23 Acero, F., Ackermann, M., Ajello, M., et al. 2016, ApJS, 223, 26 Ackermann, M., Ajello, M., Albert, A., et al. 2012a, ApJS, 203, 4 Ackermann, M., Ajello, M., Albert, A., et al. 2016, ApJL, 823, L2 Ackermann, M., Ajello, M., Allafort, A., et al. 2012b, Sci, 338, 1190 Ackermann, M., Ajello, M., Asano, K., et al. 2011, ApJ, 729, 114 Ackermann, M., Ajello, M., Asano, K., et al. 2013a, ApJ, 763, 71 Ackermann, M., Ajello, M., Asano, K., et al. 2013b, ApJS, 209, 11 Ackermann, M., Ajello, M., Asano, K., et al. 2014, Sci, 343, 42 Ackermann, M., Ajello, M., Baldini, L., et al. 2010a, ApJL, 717, L127 Ackermann, M., Asano, K., Atwood, W. B., et al. 2010b, ApJ, 716, 1178 Actis, M., Agnetta, G., Aharonian, F., et al. 2011, ExA, 32, 193 Ahlgren, B., Larsson, J., Nymark, T., Ryde, F., \& Pe'er, A. 2015, MNRAS, 454, L31

Ajello, M., Allafort, A., Axelsson, M., et al. 2018, ApJ, 861, 85 Arimoto, M., Asano, K., Ohno, M., et al. 2016, ApJ, 833, 139 Asano, K., Guiriec, S., \& Mészáros, P. 2009, ApJL, 705, L191
Atwood, W., Albert, A., Baldini, L., et al. 2013a, arXiv:1303.3514 Atwood, W. B., Abdo, A. A., Ackermann, M., et al. 2009, ApJ, 697, 1071 Atwood, W. B., Baldini, L., Bregeon, J., et al. 2013b, ApJ, 774, 76 Axelsson, M., Baldini, L., Barbiellini, G., et al. 2012, ApJL, 757, L31 Band, D., Matteson, J., Ford, L., et al. 1993, ApJ, 413, 281

Band, D. L., Axelsson, M., Baldini, L., et al. 2009, ApJ, 701, 1673 Barthelmy, S. D., Barbier, L. M., Cummings, J. R., et al. 2005, SSRv, 120, 143 Barthelmy, S. D., Kennea, J., \& Racusin, J. 2012, GCN, 13381, 1 Beloborodov, A. M. 2002, ApJ, 565, 808

Beloborodov, A. M. 2005, ApJL, 618, L13

Beloborodov, A. M., Hascoët, R., \& Vurm, I. 2014, ApJ, 788, 36

Benjamini, Y., \& Hochberg, Y. 1995, Journal of the Royal Statistical Society. Series B (Methodological), 57, 289

Bennett, C. L., Larson, D., Weiland, J. L., \& Hinshaw, G. 2014, ApJ, 794, 135 Bhat, P. N., Meegan, C. A., von Kienlin, A., et al. 2016, ApJS, 223, 28

Bissaldi, E., Omodei, N., Vianello, G., Kocevski, D. \& on behalf of the FermiLAT Collaboration 2017, in Proc. Science, 312, ed. J. Greiner \& J. McEnery, 5

Bošnjak, Ž., Daigne, F., \& Dubus, G. 2009, A\&A, 498, 677

Burgess, J. M., Bégué, D., Ryde, F., et al. 2016, ApJ, 822, 63

Burrows, D. N., Hill, J. E., Nousek, J. A., et al. 2005, SSRv, 120, 165

Cenko, S. B., Bloom, J. S., Morgan, A. N., \& Perley, D. A. 2009, GCN, 9053,

Chernoff, H. 1954, Ann. Math. Statist., 25, 573

Chevalier, R. A., \& Li, Z.-Y. 2000, ApJ, 536, 195

Chornock, R., Perley, D. A., Cenko, S. B., \& Bloom, J. S. 2009, GCN, 9028, 1 Ciprini, S., Thompson, D. J. \& on behalf of the Fermi LAT Collaboration 2013, arXiv:1303.4054

Connaughton, V., Briggs, M. S., Goldstein, A., et al. 2015, ApJS, 216, 32

Corsi, A., Guetta, D., \& Piro, L. 2010, ApJ, 720, 1008

Cucchiara, A., Fox, D., Levan, A., \& Tanvir, N. 2009a, GCN, 10202, 1

Cucchiara, A., \& Fox, D. B. 2010, GCN, 10606,

Cucchiara, A., Fox, D. B., Cenko, S. B., Tanvir, N., \& Berger, E. 2009b, GCN, 10031, 1

Cucchiara, A., Fox, D. B., Tanvir, N., \& Berger, E. 2009c, GCN, 9873, 1

Cummings, J. R., \& Palmer, D. M. 2013, GCN, 14257, 1

Daigne, F., Bošnjak, Ž., \& Dubus, G. 2011, A\&A, 526, A110

de Ugarte Postigo, A., Campana, S., Thöne, C. C., et al. 2013a, A\&A, 557, L18 de Ugarte Postigo, A., Fynbo, J. P. U., Thoene, C., et al. 2015a, GCN, 17583, 1 de Ugarte Postigo, A., Jakobsson, P., Malesani, D., et al. 2009, GCN, 8766, 1 de Ugarte Postigo, A., Kann, D. A., Thoene, C. C., et al. 2017, GCN, 20990, 1 
de Ugarte Postigo, A., Thoene, C. C., Gorosabel, J., et al. 2013b, GCN, 15470,1

de Ugarte Postigo, A., Xu, D., Malesani, D., et al. 2013c, GCN, 15187, 1

de Ugarte Postigo, A., Xu, D., Malesani, D., \& Tanvir, N. R. 2015b, GCN, 17822,1

Desai, A., Ajello, M., Omodei, N., et al. 2017, ApJ, 850, 73

Duncan, R. C., \& Thompson, C. 1992, ApJL, 392, L9

Esposito, J. A., Bertsch, D. L., Chen, A. W., et al. 1999, ApJS, 123, 203

Evans, P. A., Goad, M. R., Osborne, J. P., \& Beardmore, A. P. 2016, GCN, 19927, 1

Galli, M., Marisaldi, M., Fuschino, F., et al. 2013, A\&A, 553, A33

Gao, H., Lei, W.-H., Zou, Y.-C., Wu, X.-F., \& Zhang, B. 2013, NewAR, 57,141

Gehrels, N., Chincarini, G., Giommi, P., et al. 2004, ApJ, 611, 1005

Gehrels, N., \& Razzaque, S. 2013, FrPhy, 8, 661

Ghisellini, G., Ghirlanda, G., Nava, L., \& Celotti, A. 2010, MNRAS, 403, 926

Gilmore, R. C., Bouvier, A., Connaughton, V., et al. 2013, ExA, 35, 413

Goad, M. R., Osborne, J. P., Beardmore, A. P., \& Evans, P. A. 2015, GCN, 18403, 1

Goldstein, A., Veres, P., Burns, E., et al. 2017, ApJL, 848, L14

Granot, J., Cohen-Tanugi, J., \& do Couto e Silva, E. 2008, ApJ, 677, 92

Greiner, J., Clemens, C., Krühler, T., et al. 2009, A\&A, 498, 89

Gruber, D., Goldstein, A., Weller von Ahlefeld, V., et al. 2014, ApJS, 211, 12

Gruber, D., Paciesas, W., Pelassa, V., \& Chaplin, V. 2012, GCN, 13757, 1

Hascoët, R., Daigne, F., Mochkovitch, R., \& Vennin, V. 2012, MNRAS, 421,525

Inoue, S., Granot, J., O'Brien, P. T., et al. 2013, APh, 43, 252

Ito, H., Nagataki, S., Matsumoto, J., et al. 2014, ApJ, 789, 159

Kankare, E., O’Neill, D., Izzo, L., et al. 2017, GCN, 22002, 1

Katz, J. I., \& Piran, T. 1997, ApJ, 490, 772

Kocevski, D., Kennea, J. A., Lien, A. Y., Page, K. L., \& Racusin, J. L. 2015, GCN, 17810, 1

Kouveliotou, C., Meegan, C. A., Fishman, G. J., et al. 1993, ApJL, 413, L101 Kruehler, T., Greiner, G., \& A, K. D. 2010, GCN, 14500, 1

Kruehler, T., Schady, P., Greiner, J., \& Tanvir, N. R. 2017, GCN, 20686, 1

Kumar, P., \& Barniol Duran, R. 2009, MNRAS, 400, L75

Landsman, W., de Pasquale, M., Kuin, P., et al. 2008, GCN, 8601, 1

Lebrun, F., Leray, J. P., Lavocat, P., et al. 2003, A\&A, 411, L141

Leloudas, G., Fynbo, J. P. U., Schulze, S., et al. 2013, GCN, 14983, 1

Lien, A., Sakamoto, T., Barthelmy, S. D., et al. 2016, ApJ, 829, 7

Lithwick, Y., \& Sari, R. 2001, ApJ, 555, 540

Malesani, D., de Ugarte Postigo, A., de Pasquale, M., et al. 2016, GCN, 19708, 1

Malesani, D., Goldoni, P., Fynbo, J. P. U., et al. 2009, GCN, 9942, 1

Maselli, A., Melandri, A., Nava, L., et al. 2014, Sci, 343, 48

Mattox, J. R., Bertsch, D. L., Chiang, J., et al. 1996, ApJ, 461, 396

Meegan, C., Lichti, G., Bhat, P. N., et al. 2009, ApJ, 702, 791

Mészáros, P., Ramirez-Ruiz, E., \& Rees, M. J. 2001, ApJ, 554, 660

Metzger, B. D., Giannios, D., Thompson, T. A., Bucciantini, N., \& Quataert, E. 2011, MNRAS, 413, 2031

Milne, P. A., \& Cenko, S. B. 2011, GCN, 11708, 1

Mirzoyan, R. 2019, ATel, 12390
Moretti, E., \& Axelsson, M. 2016, MNRAS, 458, 1728

Nava, L., Vianello, G., Omodei, N., et al. 2014, MNRAS, 443, 3578

Nemiroff, R. J., Connolly, R., Holmes, J., \& Kostinski, A. B. 2012, PhRvL, 108,231103

Norris, J. P. 2002, ApJ, 579, 386

Norris, J. P., Nemiroff, R. J., Bonnell, J. T., et al. 1996, ApJ, 459, 393

Planck Collaboration, Ade, P. A. R., Aghanim, N., et al. 2016, A\&A, 594, A13

Preece, R., Burgess, J. M., von Kienlin, A., et al. 2014, Sci, 343, 51

Pugliese, V., Xu, D., Tanvir, N. R., et al. 2015, GCN, 17672, 1

Racusin, J. L., Burns, E., Goldstein, A., et al. 2017, ApJ, 835, 82

Racusin, J. L., Burrows, D. N., D’Elia, V., et al. 2012, GCN, 13845, 1

Rau, A., McBreen, S., \& Kruehler, T. 2009, GCN, 9353, 1

Razzaque, S. 2010, ApJL, 724, L109

Razzaque, S., Dermer, C. D., \& Finke, J. D. 2010, OAJ, 3, 150

Razzaque, S., Mészáros, P., \& Zhang, B. 2004, ApJ, 613, 1072

Roming, P. W. A., Kennedy, T. E., Mason, K. O., et al. 2005, SSRv, 120, 95

Ryde, F., Axelsson, M., Zhang, B. B., et al. 2010, ApJL, 709, L172

Sanchez-Ramirez, R., Gorosabel, J., Castro-Tirado, A. J., Cepa, J., \& Gomez-Velarde, G. 2013, GCN, 14685, 1

Sari, R. 1997, ApJL, 489, L37

Sari, R., Piran, T., \& Narayan, R. 1998, ApJL, 497, L17

Scargle, J. D., Norris, J. P., Jackson, B., \& Chiang, J. 2013, ApJ, 764, 167

Shao, L., Xiao, Z., \& Ma, B.-Q. 2010, APh, 33, 312

Spitkovsky, A. 2006, ApJL, 648, L51

Tak, D., Omodei, N., Uhm, L., et al. 2019, ApJ, submitted

Tanvir, N. R., \& Ball, J. 2012, GCN, 13532, 1

Tanvir, N. R., Levan, A. J., Cenko, S. B., et al. 2016, GCN, 19419, 1

Tanvir, N. R., Wiersema, K., Levan, A. J., et al. 2012, GCN, 13441, 1

Tanvir, N. R., Wiersema, K., Levan, A. J., Cenko, S. B., \& Geballe, T. 2011, GCN, 12225, 1

The CTA Consortium 2019, Science with the Cherenkov Telescope Array (Singapore: World Scientific Publishing Co. Pte. Ltd.)

Thompson, C., \& Madau, P. 2000, ApJ, 538, 105

Toma, K., Wu, X.-F., \& Mészáros, P. 2009, ApJ, 707, 1404

Usov, V. V. 1992, Natur, 357, 472

Vasileiou, V., Jacholkowska, A., Piron, F., et al. 2013, PhRvD, 87, 122001

Veres, P., Meegan, C., \& Mailyan, B. 2018, GCN, 23053, 1

Vianello, G., Gill, R., Granot, J., et al. 2018, ApJ, 864, 163

Vianello, G., Omodei, N., Chiang, J., \& Digel, S. 2017, ApJL, 841, L16

Vianello, G., Omodei, N. \& Fermi/LAT Collaboration 2015, arXiv:1502. 03122

von Kienlin, A., Veres, P., Roberts, O. J., et al. 2019, ApJ, 876, 89

Wang, X.-Y., Li, Z., Dai, Z.-G., \& Mészáros, P. 2009, ApJL, 698, L98

Weinberg, S. 1972, Gravitation and Cosmology: Principles and Applications of the General Theory of Relativity (Weinheim: Wiley-VCH), 688

Willingale, R., O’Brien, P. T., Osborne, J. P., et al. 2007, ApJ, 662, 1093

Winkler, C., Courvoisier, T. J.-L., Di Cocco, G., et al. 2003, A\&A, 411, L1

Xu, D., de Ugarte Postigo, A., Schulze, S., et al. 2013, GCN, 14478, 1

Xu, D., Levan, A. J., Fynbo, J. P. U., et al. 2014a, GCN, 16983, 1

Xu, D., Malesani, D., Fynbo, J. P. U., et al. 2016, GCN, 19600, 1

Xu, D., Malesani, D., Tanvir, N. R., et al. 2014b, GCN, 15645, 1

Zhang, B., Fan, Y. Z., Dyks, J., et al. 2006, ApJ, 642, 354 